\title{
Implementierung einer Schreibintervention in den Offenen Jugendstrafvollzug
}

\author{
Dissertation zur Erlangung des Doktorgrades \\ der Mathematisch-Naturwissenschaftlichen Fakultäten \\ der Georg-August-Universität zu Göttingen
}

\author{
vorgelegt von \\ Bettina Reinhold-Hurley \\ aus Neustadt am Rübenberge
}

Göttingen 2007 


\section{Danksagung}

Zunächst möchte ich mich bei Frau Prof. Dr. Birgit Kröner-Herwig für ihre Unterstützung und ihr Engagement bei der Planung und Durchführung dieser Dissertation bedanken. Besonders hervorheben möchte ich hierbei das große Vertrauen, das sie mir bei meiner Arbeit entgegenbrachte, welches sich besonders darin äußerte, dass sie mir stets viel Freiheit und Gestaltungsmöglichkeiten gegeben hat und immer offen für die von mir vorgebrachten Ideen und Vorschläge war.

Ich bedanke mich bei Frau Prof. Dr. Margarete Boos für Ihre Bereitschaft, das Korreferat zu übernehmen und für Ihre mir stets zugewandte Haltung. Ebenso bedanke ich mich bei Marlenna Studtmann-Pieper für Ihre Arbeit und Unterstützung im Rahmen dieses Projektes.

Mein ganz besonderer Dank gilt allen meinen KollegInnen der Abteilung für Klinische Psychologie, insbesondere Antonia Barke, die mir in unzähligen Situationen zur Seite stand und mich durch ihre Vernunft und rationale Herangehensweise an alle Lebenslagen jederzeit konstruktiv unterstützte.

Nicht zuletzt gilt mein aufrichtiger Dank allen Teilnehmern dieser Studie, die sich während ihrer Haftzeit auf das „Experiment“ einließen und ohne die das „Schreibprojekt“ gar nicht möglich gewesen wäre. Gleiches gilt für die MitarbeiterInnen der JVA Rosdorf, auf deren Unterstützung vor Ort ich mich immer habe verlassen können.

Auf diesem Weg ist es mir besonders wichtig, meinen Freunden und speziell meiner Familie zu danken. Ohne sie hätte sich mir dieser Weg nicht aufgetan. Ihre Hilfe und Unterstützung äußerte sich auf so vielen Ebenen: ob es sich nun um die Betreuung meiner Tochter, die fachliche Auseinandersetzung mit wissenschaftlichen Themen oder um ein stets offenes Ohr für meine Belange und Nöte handelte, ich weiß sicher, wem ich das alles zu verdanken habe ...

Nicht zuletzt meinen Eltern, die mit ihrer Liebe den Grundstein für mein Leben gelegt haben und dabei meinem Vater, dessen unglücklicher Tod im letzten Jahr mich unsagbar schmerzt, der aber sicherlich stolz auf alle seine Kinder gewesen wäre.

Diese Dissertation wurde von der Friedrich-Naumann-Stiftung mit Mitteln des Bundesministeriums für Bildung und Forschung gefördert. Für ihre ideelle und materielle Förderung bin ich besonders dankbar. 


\section{Inhaltsverzeichnis}

$\begin{array}{ll}\text { 1. Einleitung } & 6\end{array}$

2. Theoretischer und empirischer Hintergrund $\quad 8$

2.1 Intramurale Interventionen bei jugendlichen $\begin{array}{lr}\text { und heranwachsenden Inhaftierten } & 8\end{array}$

2.1.1 Aspekte der Klientel $\quad 8$

2.1.1.1 Psychische und physische Gesundheit 10

2.1.1.2 Therapiebedürftigkeit und -motivation $\quad 13$

$\begin{array}{ll}2.1 .2 \text { Institutionelle Bedingungen } & 15\end{array}$

2.1.2.1 Resozialisierung und Rückfälligkeit 15

2.1.2.2 Die Besonderheiten des therapeutischen Settings
im Strafvollzug

2.1.2.3 Überblick über gängige Behandlungsmaßnahmen 18

2.1.2.4 Aufarbeitung der Straftat 24

2.1.3 Wirksamkeit intramuraler Interventionsmaßnahmen 25

$\begin{array}{ll}\text { 2.2 Expressive Schreibintervention: Stand der Forschung } & 28\end{array}$

2.2.1 Das Schreibparadigma und seine Anwendungen 28

2.2.2 Erklärungsmodelle zur Wirkung Expressiven Schreibens $\quad 32$

2.2.2.1 Inhibition und Exposition 32

2.2.2.2 Kognitive und linguistische Prozesse 33

2.2.2.3 Emotionsregulation 34

2.2.2.4 Soziale Prozesse 36

2.2.3 Die Wirksamkeit des Expressiven Schreibens -
metaanalytische Befunde

2.2.3.1 Ergebnisse zu kurzfristigen Parametern 36

2.2.3.2 Ergebnisse zu langfristigen Parametern 37

2.2.3.3 Ergebnisse zu Moderatoren 42

2.2.4 Expressives Schreiben als intramurale Interventionsmaßnahme $\quad 45$

\subsection{Ziele der Studie}

2.3.1 Adaptation und Implementierung der Schreibintervention im Jugendvollzug

2.3.2 Hypothesen

3.1.1 Versuchsplanung $\quad 51$

3.1.2 Durchführung $\quad 53$

3.1.3 Erhebungszeitpunkte $\quad 55$ 
3.3 Abhängige Variablen $\quad 59$

3.3.1 Körperliche und psychische Gesundheit $\quad 59$

3.3.1.1 Krankheitstage, Arztbesuche und Substanzgebrauch $\quad 59$

$\begin{array}{ll}\text { 3.3.1.2 Körperliche Beschwerden } & 60\end{array}$

$\begin{array}{ll}\text { 3.3.1.3 Psychische Symptombelastung } & 60\end{array}$

3.3.1.4 Belastungsreaktionen $\quad 61$

3.3.2 Maße der Emotionsregulation $\quad 62$

3.3.2.1 Positiver und negativer Affekt (kurz- und langfristig) 62

$\begin{array}{ll}\text { 3.3.2.2 Depressivität } & 63\end{array}$

3.3.2.3 Zustandsangst und -ärger $\quad 63$

3.3.3 Maße der Normorientierung und Führung $\quad 65$

3.3.3.1 Normorientierung und Verantwortungsübernahme $\quad 65$

$\begin{array}{ll}\text { 3.3.3.2 Führung während der Haftzeit } & 67\end{array}$

$\begin{array}{ll}3.4 \text { Moderatorvariablen } & 68\end{array}$

3.4.1 Alexithymie $\quad 68$

3.4.2 Antisoziale Persönlichkeitsstörung $\quad 69$

$\begin{array}{ll}3.4 .3 \text { Essaymerkmale } & 69\end{array}$

$\begin{array}{ll}\text { 4. Ergebnisse } & 70\end{array}$

$\begin{array}{ll}\text { 4.1 Anwendbarkeit der Schreibintervention } & \mathbf{7 0}\end{array}$

$\begin{array}{ll}\text { 4.1.1 Merkmale der Stichprobe } & 70\end{array}$

4.1.2 Kurzfristige affektive Reaktionen $\quad 72$

$\begin{array}{ll}\text { 4.1.3 Inhalte der Essays } & 73\end{array}$

4.1.4 Bewertung des Schreibens $\quad 75$

$\begin{array}{ll}\text { 4.2 Langfristige Effekte Expressiven Schreibens } & 77\end{array}$

4.2.1 Veränderungen in Gesundheitsvariablen $\quad 78$

4.2.2 Veränderungen der Emotionsvariablen $\quad 80$

4.2.3 Veränderungen in Maßen der Normorientierung und
der Führung

4.2.4 Einflüsse von Moderatorvariablen 86

4.3 Explorative Untersuchung weiterer potentieller Einflussgrößen $\quad 91$

4.3.1 Kurzfristiger Affekt $\quad 91$

4.3.2 Soziodemografische Merkmale $\quad 92$

4.3.2 Kriminogene und haftspezifische Faktoren 94 
5.1 Anwendbarkeit und Wirksamkeit Expressiven Schreibens im Jugendvollzug

5.1.1 Kurzfristige Effekte der Schreibintervention 98

5.1.2 Längerfristige Wirkung auf körperliche und psychische Gesundheit 99

5.1.3 Längerfristige Wirkung auf Maße der Normorientierung und Führung 101

5.1.4 Moderatoreffekte

5.2 Schlussfolgerung und Ausblick

5.2.1 Expressives Schreiben als sinnvolle Methode in der Arbeit mit jugendlichen Inhaftierten? 


\section{Einleitung}

Mit belastenden Lebensereignissen wie beispielsweise dem Tod einer nahe stehenden Person, Krankheit oder Unfall ist jeder Mensch im Laufe seines Lebens mehr oder weniger häufig konfrontiert. Wie im individuellen Fall auf solche Erlebnisse reagiert wird und auf welche Weise sie im nachhinein bewältigt werden, kann sehr unterschiedlich ausfallen. Manche Menschen bereitet der Umgang mit extrem traumatischen Erfahrungen erstaunlich wenig Schwierigkeiten, bei anderen kann es nach alltäglichen Belastungssituationen und andauerndem Stress zu schweren psychischen Beeinträchtigungen oder körperlichen Erkrankungen kommen.

Da viele solcher Erlebnisse aus Scham oder Angst vor negativen Reaktionen anderer für sich behalten werden, spricht sich der Sozialpsychologe J.W. Pennebaker dafür aus, die eigenen Gefühle und Gedanken bezüglich eines belastenden Lebensereignisses wiederholt und ohne das Feedback anderer aufzuschreiben. Das so genannten Expressiven Schreiben (ES) ist seit Mitte der 80er Jahre im Rahmen unterschiedlicher Studien wiederholt mit verschiedenen Probandengruppen eingesetzt worden. Dabei wurde angenommen, dass sich das Schreiben vermittelt über emotionale, kognitive und soziale Prozesse - langfristig positiv auf die psychische und körperliche Gesundheit auswirkt. Die Instruktion, sich auf emotional expressive Weise schriftlich mit einem persönlich belastenden Thema auseinander zu setzen, wurde in verschiedenen Studien schnell auf andere Lebensthemen, wie etwa dem Schreiben über persönliche Lebensziele, Partnerschaft oder gar positive Lebensereignisse ausgeweitet.

In der hier vorliegenden Studie wird das ES erstmals mit jugendlichen und heranwachsenden Inhaftierten einer offenen Jugendanstalt durchgeführt. Es kann davon ausgegangen werden, dass gerade diese spezielle Klientel belastende Lebensereignisse (wie z.B. Heimunterbringung, Gewalt in der Familie, Drogenkonsum) aus ihrer Vorgeschichte zu berichten hat und sich durch die Tatsache der (erstmaligen) Inhaftierung in einer völlig neuen Lebens- und Anpassungssituation befindet. In diesem Zusammenhang soll untersucht werden, inwieweit sich die Wirksamkeit der von Pennebaker vorgeschlagenen Schreibintervention im Inhaftierungskontext mit jugendlichen Inhaftierte nachweisen lässt. Neben dem Schreiben über ein belastendes Lebensereignis soll die ursprüngliche Instruktion auf straftatsrelevante Aspekte, die somit von legalprognostischer Bedeutung sein können, übertragen werden. Eine Gruppe Inhaftierter schreibt dabei auf emotional expressive Weise über die vergangene Straftat und setzt sich - angelehnt an das Prinzip der Straftataufarbeitung - mit der eigenen kriminellen 
Vergangenheit auseinander. Es wird angenommen, dass diese Reflektion außerdem die Möglichkeit bietet, positive Veränderungen in den Bereichen Normorientierung, Verantwortungsübernahme und Führungsverhalten während der Haftzeit zu bewirken.

Des weiteren soll der Frage nachgegangen werden, inwieweit eine mögliche Wirksamkeit der Schreibintervention von bestimmten Persönlichkeitseigenschaften der Probenden und dem gewählten Schreibstil beeinflusst wird. 


\section{Theoretischer und empirischer Hintergrund}

\subsection{Intramurale Interventionen bei jugendlichen und heranwachsenden Inhaftierten}

Die Jugendstrafe ist in erster Linie Erziehungsstrafe. Das Jugendstrafrecht gibt dem Strafvollzug zwei Handlungsrichtungen. Mit Hilfe von Erziehung und/oder Behandlung soll eine Änderung im Verhalten bei Straftätern mit „schwer gestörter Sozialisation“ erreicht werden, damit diese nach ihrer Entlassung in der Lage sind, soziale Normen zu akzeptieren und nach ihnen zu leben (Mey, 1996, S.118). So genannte Konflikttäter, die eine schwere Straftat unter extremer Konfliktbelastung begangen haben, müssen hingegen ihren „Sozialisationsstand“ im Jugendvollzug erhalten und bewahren können. Generell gilt, dass alle Insassen des Jugendstrafvollzuges (ebenso wie erwachsene Inhaftierte) vor den schädlichen Folgen eines Freiheitsentzuges zu schützen sind (§ 3 Abs. 2 StVollzG).

Die „Erziehung“ jugendlicher und heranwachsender Strafgefangener erfolgt laut Jugendgerichtsgesetz (JGG) über verschiedene Maßnahmen, wie etwa Unterricht, Förderung beruflicher Leistungen oder sinnvolle Beschäftigung in der Freizeit (§91 Abs. 2 JGG). Der Vollzug kann aufgelockert und in „freien Formen“ durchgeführt werden, was der generellen Gestaltung einer Jugendhaftanstalt verhältnismäßig viel Freiraum bietet. Dies erleichtert die Umsetzung eines wesentlichen Ziels des Jugendstrafvollzuges, nämlich die Konfliktbewältigungsfähigkeit jugendlicher und heranwachsender Inhaftierter zu stärken, um sie sozial leistungsfähiger und kompetenter werden zu lassen (Mey, 1996).

Bevor die theoretische und empirische Grundlage der im Rahmen dieser Studie zum Einsatz gelangten Methode des Expressiven Schreibens dargestellt wird, wird im folgenden Abschnitt zunächst ein Überblick über die häufiger eingesetzten intramuralen Interventionsmaßnahmen (national und international) und ihre Wirksamkeit gegeben. Für ein besseres Verständnis der Ziele dieser Maßnahmen ist es sinnvoll, sich zuvor die Besonderheiten der Klientel und des speziellen Settings des Strafvollzuges zu vergegenwärtigen.

\subsubsection{Aspekte der Klientel}

Die Verhängung einer Jugendstrafe wird gemäß $§ 17$ (JGG) auf solche Täter beschränkt, die entweder ein „extrem sozial abweichendes Verhalten mit chronifizierter krimineller Lebensbewältigungstechnik“ aufweisen oder aber schwerwiegende Straftaten begangen haben (Mey, 1996, S. 117). Für die erstgenannte Gruppe ist im Gesetzestext die Rede von so genannten „schädlichen Neigungen des Jugendlichen, die in der Tat hervorgetreten sind“. Gleichzeitig 
reichen „Erziehungsmaßregeln oder Zuchtmittel zur Erziehung nicht“ aus, so dass (als „ultima Ratio“) eine freiheitsentziehende Maßnahme mit „erforderlicher erzieherischer Einwirkung“ zu verhängen ist ( $\S 17$ Abs. 2, 18 Abs. 2 JGG). Im Fall der Gruppe der „Konflikttäter“ liegen Straftaten mit sehr schwerem Schuldgehalt vor, so dass auch ohne das personenbezogene Merkmal „dauerhafte schädliche Neigung“ eine Jugendstrafe verhängt wird (Mey, 1996, S.117). Offensichtlich handelt es sich demnach bei jugendlichen Strafgefangenen um eine hoch ausgelesene Gruppe, bei der alle anderen bisher angewandten Maßregeln nicht oder nicht ausreichend hilfreich gewesen sind. Im Rahmen einer Studie im bayrischen Justizvollzug konnte gezeigt werden, dass inhaftierte Jugendliche im Schnitt sechsmal vorm Richter gestanden haben, bevor gegen sie eine (längere) Jugendstrafe ohne Bewährung verhängt wurde (Hochweber, 2004). Die Rückfallquote nach der Entlassung aus dem Jugendstrafvollzug liegt mit etwa $80 \%$ höher als die erwachsener Inhaftierter, wobei es bei 50-60\% der entlassenen Jugendlichen zu einer erneuten Inhaftierung kommt (Dünkel \& Drenkhahn, 2001). Diese vergleichsweise hohen Rückfallraten sind unter der Berücksichtigung der delinquenten Entwicklung über die Lebensspanne damit zu erklären, dass ein erheblicher Anteil der rückfälligen Jugendlichen der so genannten ,jugendbegrenzten Delinquenz“ (im Gegensatz zu chronisch delinquentem Verhalten) zuzuordnen ist. Diese Gruppe verliert mit Eintritt ins Erwachsenenalter ihr delinquentes Verhalten und tritt zumeist nicht mehr strafrechtlich in Erscheinung (Dahle, 2005).

In der Forschung zur Persönlichkeits- und Delinquenzentwicklung konnten neben den bekannten situativen Faktoren, die (jugendliche) Delinquenz begünstigen, wie beispielsweise familiäre und soziokulturelle Faktoren oder psychosozialer Stress auch zeitlich stabile Persönlichkeitsmerkmale nachgewiesen werden, die im Hinblick auf die Delinquenzentwicklung als bedeutsam angesehen werden. Persönlichkeitseigenschaften, die das Risiko für Delinquenz erhöhen und bereits frühzeitige Prädiktoren darstellen, sind Impulsivität (niedrige Selbstkontrolle), geringe Verlässlichkeit/Persistenz, Abenteuerlust, geringe Frustrationstoleranz sowie Indifferenz (Cauffman \& Steinberg, 2000; Gottfredson \& Hirschi, 1990; Lewand, 2005). Die Klientel jugendlicher Inhaftierter ist darüber hinaus durch soziale Merkmale gekennzeichnet wie einer hohen Verwaisungsrate (etwa ein Drittel ganz oder teilweise), Schulversagen (ein Drittel) sowie fehlender Berufsausbildung bei der Hälfte derjenigen, die nicht mehr Schüler sind (Enzmann \& Greve, 2001). Häufig finden sich Defizite im Intelligenzbereich sowie vermehrter Alkohol- und Drogenkonsum vor und während der Haft (Greve, Hosser \& Pfeiffer, 1996). Bezüglich der Deliktstruktur jugendlicher und heranwachsender Inhaftierter weisen Delikte wie Diebstahl (95\%) und Körperverletzung (77\%) gefolgt von Sachbeschädigung 
(50\%) und Raub (50\%) die höchsten Prävalenzraten auf. Enzmann \& Greve (2001) fassen die Ergebnisse ihrer Studie ${ }^{1}$ über soziale und individuelle Merkmale jugendlicher Strafgefangener wie folgt zusammen: Die Klientel jugendlicher und heranwachsender Inhaftierter ist gekennzeichnet von einer „extreme[n] sozialen Benachteiligung auf mehreren Ebenen“ (S. 140). So zeigen sich massive Defizite der familiären Sozialisation, wie unvollständige Familien, Heimaufenthalte, hohe Raten von Misshandlung etc., soziale und wirtschaftliche Randständigkeit der Herkunftsfamilie, wie ein geringes Bildungsniveau der Eltern oder niedriger sozioökonomischer Status, eine schlechte Schul- und Berufsausbildung der Jugendlichen selbst, eine hohe Arbeits- und Erwerbslosenrate und damit zusammenhängend ein geringes (legales) Einkommen und ein hohes Maß an Drogenkonsum.

\subsubsection{Psychische und physische Gesundheit}

Auch in Bezug auf ihren psychischen wie physischen Gesundheitsstatus hebt sich die Gruppe (jugendlicher) Inhaftierter von der gleichaltrigen Allgemeinbevölkerung ab. Behandlungs-, Erziehungs- und Interventionsansätze im Strafvollzug müssen daher in ihrer Konzeption und Durchführung die oftmals vielschichtigen Probleme ihrer Klienten berücksichtigen.

In den letzten drei Jahrzehnten sind zahlreiche internationale Forschungsarbeiten erschienen, deren Ergebnisse zeigen, dass die Raten psychischer Störungen bei inhaftierten Stichproben höher sind als die der Allgemeinbevölkerung. So liegt die allgemeine Rate psychischer Störungen (inklusive Persönlichkeitsstörungen und Substanzbezogener Störungen) bei inhaftierten Populationen im Bereich von 55\% - 80\% (vgl. Brink, 2005). Es wird geschätzt, dass in den USA jeder fünfte Inhaftierte unter einer psychiatrischen Störung leidet und daher in vielen Fällen die Doktrin der „criminalization of the mentally ill“ eine treffende Beschreibung der Umstände zu sein scheint (Abramson, 1972).

Fazel und Danesh (2002) berichten in ihrer umfangreichen Metaanalyse mit 62 Einzelstudien aus 12 verschiedenen westlichen Ländern neben einer Depressionsprävalenz von 10\% weiterhin von einer hohen Rate Antisozialer Persönlichkeitsstörung (47\%) der untersuchten (erwachsenen) Inhaftierten. Bezüglich jugendlicher Inhaftierter ergeben sich Prävalenzraten (Aktualprävalenz) psychischer Störungen, die von 61 bis 90\% reichen (Teplin, Abram \& McClelland, 2002; Vreugdenhil, Doreleijers \& Vermeiren, 2004). Von Affektiven Störungen sind 15\% der Gefangenen zum Zeitpunkt der Untersuchung betroffen. Die höchsten Raten ergeben sich erwartungsgemäß im Bereich Substanzbezogener Störungen (40 bis 50\%). Es wird ge-

\footnotetext{
${ }^{1}$ Die Ergebnisse entstammen einer Studie im Rahmen des Projekts „Gefängnis und die Folgen“ (1998-2000), bei der ca. 2500 jugendliche und heranwachsende Erstinhaftierte in fünf norddeutschen Jugendhaftanstalten befragt wurden.
} 
schätzt, dass etwa die Hälfte aller Drogenkonsumenten ihren Konsum während der Haft fortsetzen (Stöver, 1999). Im Rahmen einer Untersuchung in deutschen Jugendanstalten wurden 20\% der Erstinhaftierten als alkoholabhängig bzw. -missbrauchend und 24\% als drogenabhängig bzw. -missbrauchend diagnostiziert (Enzmann \& Raddatz, 2005). Aber auch Anpassungsstörungen (hierzu sind auch die so genannten „Haftreaktionen“, „Inhaftierungsschock“ und „Haftkoller“ zu zählen) sowie Störungen der Aufmerksamkeit, der Aktivität und des Sozialverhaltens (z.B. Störung mit Oppositionellem Trotzverhalten) finden sich bei knapp 40\% männlicher jugendlicher Inhaftierter (Gosden, Kramp, Gabrielsen \& Sestoft, 2003; Konrad, 2004; Teplin et al., 2002; Vreugdenhil et al., 2004). Das Maß an Lernschwächen (IQ< 70) jugendlicher männlicher und weiblicher Delinquenter wird auf 20\% geschätzt (Chitsabesan et al., 2006).

In einer Studie von Abram, Teplin und Charles (2004) wurden erstmals jugendliche (Untersuchungs-) Häftlinge im Alter von 10 bis 18 Jahren systematisch hinsichtlich möglicher akuter Belastungssymptomatik und vergangener traumatischer Erlebnisse untersucht. Über $90 \%$ der Stichprobe berichtete über mindestens ein traumatisches Erlebnis aus der Vergangenheit. Bezogen auf das vergangene Jahr erfüllten 11\% der Befragten die Kriterien einer Posttraumatischen Belastungsstörung. Im Mittel gaben die befragten jugendlichen Straftäter knapp 15 traumatische Erlebnisse zu Protokoll, wobei männliche Inhaftierte durchschnittlich von höheren Raten berichteten als weibliche. Die am häufigsten berichteten Erlebnisse beinhalteten das Beobachten von Gewalt oder Tod, die Bedrohung der eigenen Person durch eine Waffe oder die Erwartung, dass ein anderer oder man selbst verletzt wird oder zu Tode kommt. Im Vergleich zur Allgemeinbevölkerung ist bei diesen Jugendlichen eine weitaus höhere Belastung durch Traumata zu finden. Die Autoren kommen zu dem Schluss ,exposure to trauma is a fact of life for delinquent youth...yet services are insufficient" (S. 407).

Bei den Angaben einiger Autoren der zitierten Studien ist einschränkend zu erwähnen, dass unklar bleibt, ob es sich bei den genannten Raten im einzelnen um Aktual- oder aber um Lebenszeitprävalenzen handelt, bzw. inwieweit die gleichen Zeitfenster definiert worden sind. Zudem ergeben sich methodische Probleme aus der Verwendung heterogener diagnostischer Instrumente (Klinische Interviews, Fragebögen, Aktensichtung). Dennoch bleibt unbestritten, dass die Vulnerabilität (jugendlicher) Inhaftierter für psychische Störungen bei weitem höher ist als bei Gleichaltrigen der Allgemeinbevölkerung (Fazel \& Lubbe, 2005; Konrad, 2004). Sheldon (2001) schätzt, dass 26\% jugendlicher und heranwachsender Häftlinge eine sofortige psychosoziale Intervention benötigen. 
Im Rahmen einer Erklärung für die erhöhte Prävalenz psychischer Störungen im Justizvollzug werden verschiedene relevante Faktoren diskutiert. Zum einen sind ursprüngliche Risikofaktoren für Delinquenz oftmals die gleichen wie für psychische Probleme, wie etwa inkonsistenter elterlicher Erziehungsstil oder Missbrauchserfahrungen. Zum anderen können viele Aspekte von Kriminalität und kriminellem Verhalten im Sinne psychischer Stressoren wirken, wie z.B. Gewalterfahrungen, riskantes und selbstgefährdendes Verhalten (Hagell, 2002).

Neben dem bereits erwähnten Effekt der Kriminalisierung psychisch Kranker, nach dem sozial abweichendes Verhalten nicht toleriert und zur Anzeige gebracht wird, werden auf der institutionellen Ebene Faktoren wie die Ökonomisierung der Behandlung psychisch Kranker mit dem Abbau stationärer Langzeiteinrichtungen, verkürzter Behandlungsdauer und unzureichender Entlassungsvorbereitung als Erklärung für die belastete psychische Gesundheit Inhaftierter genannt. Gleichzeitig bestehen nur unzureichende komplementäre Versorgungsstrukturen in der Gemeinde (z.B. für jugendliche psychiatrische Patienten). Bei auffälligem und nicht gesetzeskonformen Verhalten sowie bestehender Behandlungsnotwendigkeit ohne Behandlungsbereitschaft kommt dem Justizvollzug überdies die Rolle zu, eine „Sicherungslücke“ zu schließen, die mangels sonstiger sozialer Kontrolle und „eng gefassten Kriterien zivilrechtlicher oder öffentlich-rechtlicher Unterbringung“" offen bleibt (Konrad, 2004, S. 322).

Studien zur körperlichen Gesundheit Inhaftierter liegen in geringerem Umfang vor. Untersuchungen aus den USA kritisieren die gesundheitliche Unterversorgung in der Behandlung von jugendlichen Delinquenten und ihren damit einhergehenden schlechteren Gesundheitsstatus im Vergleich zur Allgemeinbevölkerung. Wobei sich die medizinische Unterversorgung vor allem auf das Umfeld außerhalb des Strafvollzuges bezieht. Oft besteht überhaupt erst mit der Inhaftierung die Möglichkeit, sich fachärztlich untersuchen und versorgen zu lassen (Golzari, Hunt \& Anoshiravani, 2006). Daher ist es eine wichtige Aufgabe im Rahmen der Entlassungsvorbereitung, die Schwelle ehemalig Inhaftierter abzubauen, Kontakt zu Einrichtungen der Gesundheitsversorgung aufzunehmen und ihnen den Zugang generell zu erleichtern. Im Rahmen einer Befragung australischer (erwachsener) Inhaftierter zum subjektiven Gesundheitsstatus, gaben 65\% der männlichen Stichprobe an, unter mindestens einem chronischen Leiden zu leiden und $41 \%$ gaben an, mehreren chronischen Krankheiten zu haben. Die am häufigsten genannten Leiden sind Rückenprobleme, schlechtes Sehvermögen, Arthritis, Bluthochdruck und Asthma (Butler, Kariminia, Levy \& Murphy, 2004). Die Ergebnisse einer Metaanalyse, die den Gesundheitsstatus der Insassen britischer Haftanstalten zusammenfasst, zeigen, dass insbesondere jüngere Gefangene häufig einen gesundheitsgefährdenden Lebens- 
stil aufweisen. Im Vergleich zu nicht-inhaftierten Gleichaltrigen konsumieren sie mehr Tabak, Alkohol und illegale Drogen, treiben weniger Sport, haben früher sexuelle Kontakte und sind häufiger von sexuell-übertragbaren Krankheiten betroffen (Harris, Hek \& Condon, 2006). Es finden sich erhöhte Raten an abnormaler Leberfunktion und chronischer Hepatitis (Golzari et al., 2005). Ähnlich wie im Erwachsenenvollzug weisen $40 \%$ der 16 bis 24 jährigen Inhaftierten eine anhaltende Krankheit oder Beeinträchtigung auf. 21\% sind von Atemwegsproblemen betroffen. Ein Viertel der Population gibt im Selbstbericht an, unter einer längerfristigen körperlichen Beeinträchtigung zu leiden (Lader, Singelton \& Meltzer, 2000). In einer Untersuchung von Hein, Cohen und Litt (1980) gaben 11\% der jugendliche Inhaftierten an, unter Kopfschmerzen zu leiden, gefolgt von Nebenhöhlenverstopfung (5,2\%), Rücken- und Gelenksschmerzen (4,5\%) und Schmerzen in der Brust (3,7\%). In einer anderen Studie konnten bei längerfristig untergebrachten jugendlichen Straftätern am häufigsten Hautkrankheiten (44\%) und Atemwegserkrankungen (35,6\%) diagnostiziert werden (Anderson \& Farrow, 1998).

\subsubsection{Therapiebedürftigkeit und -motivation}

Untersuchungen zum Verlauf psychischer Störungen und psychosozialer Beeinträchtigungen jugendlicher Delinquenter während und nach der Inhaftierung konnten zeigen, „there was no change in mental health needs“" (Chitsabesan et al., 2006, S. 538). Neben der häufig vorzufindenden psychosozialen Unterversorgung (jugendlicher) Inhaftierter, sind die meisten psychischen Probleme offensichtlich nicht haftspezifisch und auch nicht notwendigerweise durch den Freiheitsentzug verstärkt (Brink, 2005; Fazel \& Danesh, 2002; Fazel \& Lubbe, 2005). Wie bereits erwähnt, kann angenommen werden, dass bei knapp einem Drittel jugendlicher und heranwachsender Inhaftierter psychosozialer Interventionsbedarf besteht (Sheldon, 2006). Was die Therapiemotivation inhaftierter Straftäter angeht, so wird häufig von einer „,vermeintlich ungenügenden Motivation“ auf Seiten der Klienten gesprochen, welches nicht selten den Eindruck erweckt, auf Behandlungsversuche könne generell verzichtet werden (Dahle, 2003b, S. 231). Die Übertragung des klassischen Verständnisses von Therapiemotivation sowie die Forderung nach artikulierter Behandlungsbereitschaft durch die Klientel im Strafvollzug bringe laut Dahle einige Schwierigkeiten mit sich. Im Vergleich zum psychotherapeutischen Durchschnittsklientel stammten delinquente Personengruppen aus „sozial benachteiligten Schichten“, die „mögliche Ressentiments“ gegenüber Psychotherapie wahrscheinlich mache (S. 234). Zudem seien Vorerfahrungen mit verschiedenen sanktionierenden gesell- 
schaftlichen Institutionen zu bedenken, die Auswirkungen auf die Einstellung Inhaftierter gegenüber Therapieangeboten des Strafvollzuges haben können. In einer Studie mit 390 erwachsenen männlichen Strafgefangenen wurde in diesem Zusammenhang untersucht, inwieweit die beiden Konzepte Leidensdruck und Behandlungsbereitschaft angemessen das Therapiemotivationskonstrukt der Klientel im Strafvollzug widerspiegeln. Es ergaben sich vier mögliche Gruppen (mit den möglichen Kombinationen Leidensdruck vorhanden/nicht vorhanden und Behandlungsbereitschaft vorhanden/nicht vorhanden), wobei die Gruppe der Personen mit Leidensdruck und Behandlungsbereitschaft zahlenmäßig die größte darstellte. Ein Zusammenhang $(\mathrm{Phi}=.25)$ der beiden Komponenten ist zwar erkennbar geworden, erscheint aber geringer als das klassische Therapiemotivationskonstrukt erwarten ließ (Dahle, 2003b). Die inkonsistenten Gruppen (vorhandener Leidensdruck und gleichzeitig fehlende Behandlungsbereitschaft sowie fehlender Leidensdruck mit vorhandener Behandlungsbereitschaft) machten 36\% der Stichprobe aus. Die Studie ergab ferner, dass die zwei konsistenten Gruppen sich hinsichtlich verschiedener biografischer, sozialer und krimineller Variablen unterscheiden. So war die Gruppe der Therapiemotivierten und -bedürftigen von hoher biografischer und krimineller Belastung wie z.B. einem langem Heimaufenthalt gekennzeichnet. Testpsychologisch zeigte sich diese Gruppe ebenfalls am stärksten psychisch und psychovegetativ beeinträchtigt. Die Gruppe der Therapieunmotivierten stellte das Gegenstück dar und war sowohl testpsychologisch wie auch hinsichtlich ihres biografischen und kriminellen Hintergrundes die unauffälligste. Die inkonsistenten Gruppen nahmen in Bezug auf Störungsparameter eine Mittelstellung ein. Die Gruppe mit Leidensdruck aber ohne Behandlungsbereitschaft zeigte zwar deutliche Sozialisationsbelastungen, hatte aber Schwierigkeiten bei innerpsychischen Problemen Hilfe von außen anzunehmen und war der Meinung, dass es grundsätzlich besser sei, persönliche Probleme allein zu lösen bzw. die „Dinge laufen zu lassen“ (S. 246). Dabei trennte diese Gruppe am stärksten zwischen seelischer Befindlichkeit und kriminellen Erfahrungen. Motivationales Geschehen stellt sich somit für Dahle nicht als ein eindimensionales Geschehen dar. Das Konzept der Therapiemotivation müsse vielmehr gruppenspezifisch erweitert und individuell angepasst (z.B. mittels Berücksichtigung des Änderungsmotivs als notwendige aber nicht hinreichende Bedingung) werden, damit es ,motivische Besonderheiten dieses Personenkreises“ adäquat erfasse (Suhling, 2005, S. 247).

Im Rahmen einer Untersuchung von Hinrichs, Behnisch, Krull und Reimers (2000) zur Therapiemotivation 145 junger männlicher Strafgefangener wurden Einflussfaktoren, Struktur und Vorhersagbarkeit von Therapiemotivation erfasst. Im Gegensatz zu Dahle (2003b), ließ sich faktorenanalytisch ein eindimensionales Konstrukt von Therapiemotivation nachweisen, 
wobei diese sich aus den Variablen emotionale Labilität, Symptombelastung, Therapieerwartung und dem Ausmaß an Gehemmtheit vorhersagen ließ. Eine hohe Ausprägung dieser Variablen ließ auf eine hohe Therapiemotivation schließen. 23\% der untersuchten Stichprobe hatte bereits Erfahrung mit psychologischer Behandlung und Beratung, wobei die Hälfte von ihnen diese als hilfreich empfand (,Fremdmotivierte“ in geringerem Ausmaß als „Eigenmotivierte"). Die Therapieerwartung derjenigen, die zu einer Behandlungsmaßnahme während der Haft bereit waren, war vor allem bestimmt durch die Art der therapeutischen Beziehung, nämlich „mit jemandem reden [zu] können“, dem man vertraut (Hinrichs et al., 2000, S. 259). Die Therapieerwartungen Erstinhaftierter war bei gleichzeitig geringerem Leidensdruck höher als die Erwartungen Mehrfachinhaftierter.

Generell scheint sich jedoch das recht hohe Maß an Fremdmotiviertheit von Straftätern im Vergleich zu anderen klinischen Patientengruppen auf ihre Erfolgseinschätzung von Behandlung auszuwirken. Diese fällt bei Straftätern geringer aus und ist u.a. ein Grund für die hohe Therapieabbruchsrate von ca. 45\% (Beier \& Hinrichs, 1995; Krupinski, Schöchlin, Fischer \& Nedopil, 1998; Wendt \& Hinrichs, 1993). Für die dennoch recht häufig geäußerte Therapiebereitschaft Inhaftierter sind verschiedene Motive zu berücksichtigen, wie eine größere Vulnerabilität nach Tatbegehung und Inhaftierung, mögliche Hafterleichterung oder Nutzen der (Therapie-) Zeit (Hinrichs et al., 2000).

Eine oft notwendige und über die Haftzeit hinaus gehende therapeutische und pädagogische Betreuung jugendlicher Straftäter macht zunächst eine strukturierte Diagnostik der Probleme und Bedürfnisse dieser Klientel notwendig (Chitsabesan, 2006). Überdies ist es angebracht, im Einzelfall die Interaktion zwischen Symptomcluster und dysfunktionalen/delinquenten Verhalten zu explorieren, um geeignete Interventionsmaßnahmen abzuleiten. Zu Beginn einer Intervention geht es vor allem darum, Mitarbeitsbereitschaft herzustellen.

\subsubsection{Institutionelle Bedingungen}

\subsubsection{Resozialisierung und Rückfälligkeit}

Unter dem Begriff „Resozialisierung“ wird gemeinhin die „Wiedereingliederung Straffälliger in die Gesellschaft" verstanden (Pecher, 2004, S. 215). Neben dem Sicherheitsaspekt (Schutz der Allgemeinheit vor weiteren Straftaten) stellt die Resozialisierung das wesentliche Vollzugsziel dar, d.h. ein Gefangener soll im Vollzug fähig werden, künftig in sozialer Verant- 
wortung ein Leben ohne Straftaten zu führen ( $§ 2, \mathrm{StVollzG})$. In der Praxis werden Resozialisierungsmaßnahmen meist multiprofessionell und integrativ durchgeführt und können verschiedene Schwerpunkte aufweisen. Sie beinhalten psychotherapeutische Behandlung bei Persönlichkeitsstörungen und psychischen Problemen, soziale Trainings zur Förderung sozialer Kompetenzen und Erhöhung der Frustrationstoleranz, schulische und berufliche Bildungsmaßnahmen, Beratung bei speziellen Krisensituationen und Drogenproblematiken, persönliche Begleitung in Konflikt- und Übergangssituationen und materielle Hilfen zur Absicherung der Lebenshaltungskosten (vgl. Pecher, 2004).

Im Jugendstrafvollzug steht der bereits erwähnte Erziehungsgedanke im Vordergrund (vgl. 2.1). Er kann jedoch auf unterschiedliche Weise definiert und verstanden werden (pädagogisch, psychologisch, rechtlich), so dass die Verhinderung von Rückfallkriminalität als „,der kleinste gemeinsame Nenner“" verstanden werden kann. Daraus ergeben sich z.T. hohe Erwartungen an die Institution und es entsteht ein „Spannungsfeld von Erziehung und Strafe“ (Sonnen, 2002, S.60). Die Rückfallraten ehemaliger jugendlicher Inhaftierter sind vergleichsweise hoch. Die Wiederverurteilungsrate liegt bei etwa 84\%, knapp 60\% werden erneut inhaftiert (Kerner \& Janssen, 1996; Sonnen, 2002). Trotz der scheinbar geringen Erfolgschancen der Jugendstrafe (ohne Bewährung) haben sich die Inhaftierungszahlen im Jugendstrafvollzug seit 1992 beinah verdoppelt.

In ihrer Langzeitstudie mit 500 ehemaligen jugendlichen Häftlingen konnten Kerner und Janssen (1996) zeigen, dass aus der Vorgeschichte mehrfach belastete Jugendliche nach der Entlassung in weit höherem Maße wieder auffällig wurden und ein linearer Zusammenhang zwischen Verurteilungshöhe, Freiheitsstrafenhöhe, Verbüßungsdauer und den Belastungen aus der Vorgeschichte bis zur Einweisung besteht. Die Autoren schlussfolgern, „Resozialisierung [könne] als eine in fixierter Weise ungleich verteilte Lebenschance“ verstanden werden (S. 168). In der Studie zeigt sich überdies, dass die Anzahl an Wiederverurteilungen nach Haftentlassung im Verlauf der Zeit geringer wird und von einer ,allmählichen Entwicklung aus der Straffälligkeit heraus“ die Rede ist (S. 149). Im längerfristigen Verlauf wird demnach auch bei jüngeren Männern, die aus dem Jugendstrafvollzug entlassen sind, die Legalbewährung zur Regel. Die Daten der Studie lassen vermuten, dass die Erfahrungen aus dem Vollzugsalltag nur selten Einfluss im Sinne einer „radikalen Umgestaltung“ auf die Lebensläufe der Jugendlichen zu haben scheinen (S. 217). Es ergibt sich vielmehr das Bild, dass mit einigen Gefangenen sinnvoll und intensiv gearbeitet worden zu sein scheint und sich dennoch bei einigen von ihnen (angelegte) Probleme eher verstärkt haben. Ein Grund für den langsamen Austritt aus der Kriminalität könnte laut den Autoren darin liegen, dass viele der Strafgefan- 
genen und Entlassenen sich erst dann vollständig zu resozialisieren scheinen, wenn sich ihre persönliche und berufliche Lebenssituation stabilisiert hat und sie in der Lage sind, eigenverantwortlich zu agieren und Erfahrungen zu nutzen, die ihnen im institutionellen Rahmen des Strafvollzuges als „Integrationsangebote“ gemacht worden sind (S. 218).

\subsubsection{Besonderheiten des therapeutischen Settings im Strafvollzug}

Soweit es die (psycho-) therapeutische Behandlung im Setting des Strafvollzuges betrifft, stellt sich zunächst die Frage der Indikation. Nicht alle Straftäter sind wegen der bloßen Tatsache ihrer Inhaftierung bzw. ihrer kriminellen Vergangenheit behandlungsbedürftig. Untersuchungen diesbezüglich haben ergeben, dass etwa die Hälfte erwachsener Inhaftierter Behandlungsbereitschaft äußert (Dahle, 2003b; Stemmer-Lück, 1980). Eine vom Häftling geäuBerte Behandlungsbereitschaft ist nicht unmittelbar mit einer Indikation gleichzusetzen, die letztlich von der „Interaktion von Institutions-, Methoden- und Personenvariablen“ abhängt (Steller, 2003, S. 5). Nicht zuletzt sind bei der Indikation auch deliktspezifische Überlegungen angebracht. So spielen etwa bei Sexual- oder Gewaltdelinquenten Aspekte des Symptomcharakters der Straftat, Deliktschwere und die (potentielle) Opferschädigung für die Indikation einer (psychotherapeutischen) Intervention eine bedeutsame Rolle (Hinrichs, 1995).

Mögliche Probleme bei der Behandlung von Inhaftierten lassen sich in verschiedene Bereiche unterteilen (Dahle, 2003a). Strukturelle Probleme ergeben sich aus den strafrechtlichen Rahmenbedingungen von innervollzuglichen Behandlungsangeboten, die internal orientierte Änderungsstrategien mit eher external formulierten Behandlungsaufträgen in Einklang zu bringen haben. Darüber hinaus wird im Kontext von stationärem Straf- oder Maßregelvollzug die „Therapiefeindlichkeit“ der Institutionen angeführt (S. 179). Zunächst erscheint der gleichzeitige Auftrag der Vollzugsanstalten für den Vollzug von Strafen, für eine gesellschaftliche Sicherung und für die Behandlung Inhaftierter widersprüchlich. Diese Aufgabenvielfalt setzt die Entwicklung von Gesamtkonzepten mit einer „Gesamtzielbeschreibung“ voraus, die von den unterschiedlichen institutionellen Berufsgruppen mitgetragen werden kann und muss. Laut Dahle ist eine integrative Zielstellung und Konzeption in vielen Einrichtungen (bisher) allerdings nur in Ansätzen entwickelt, so dass eine Umsetzung von Aufgaben und Behandlung durch die „strukturellen Machtverhältnisse zwischen den Berufsgruppen“ bestimmt werde (S. 180). Zudem sei zu kritisieren, dass in der Straftäterbehandlung eine ungenügend spezifische Ausbildungssituation herrsche und bei vielen Behandlern fächerübergreifende Kenntnisse 
fehlten, die diese sich oftmals in der Praxis mühsam selbst aneignen müssten. Als methodisches Problem ergebe sich, dass viele Behandlungsmodelle der klassischen Psychotherapieschulen ursprünglich für andere Zielgruppen (zumeist mit mittlerem Bildungsniveau und „,neurotischem“ Störungsbild) mit dem Fokus auf die Modifikation innerpsychischer Konflikte entwickelt wurden und sich nicht ohne weiteres auf die Bedürfnisse der Klientel im Strafvollzug übertragen ließen. Auch fehle es an der Entwicklung spezifischer Therapiemodelle für die Behandlung delinquenter Gruppen (wie es in der Behandlung von Sexualstraftätern bereits geschieht) mit verschiedenen Störungsbildern (Rauchfleisch, 2003). Ein weiterer Problembereich bestehe in den genannten Besonderheiten der Zielgruppe, die nicht zuletzt eine gewisse Unsicherheit und Distanz gegenüber psychologischen Methoden und deren therapeutischen Sprache und Werthaltung zeigt. Für die ausführliche Darstellung der Probleme der „Zwangstherapie“", der Bedeutung der Freiwilligkeit bei der Durchführung therapeutischer Maßnahmen und der schwierigen Stellung des Therapeuten (z.B. Trennung von psychotherapeutischer Arbeit und Prognosestellung) speziell in der Arbeit mit der Klientel Sozialtherapeutischer Anstalten wird an dieser Stelle auf Egg (1986, 2003) sowie auf Brandewiede (1995) verwiesen.

\subsubsection{3 Überblick über Behandlungsmaßnahmen}

Gesetzlich besteht kein abgeschlossener Katalog von (Behandlungs-) Maßnahmen innerhalb des Strafvollzuges. So soll zum einen eine Weiterentwicklung der Methoden in der Praxis offen gehalten werden und zum anderen sollen sich Behandlungsmethoden aus dem jeweiligen (For- schungs-)stand wissenschaftlicher Erkenntnisse ergeben (Brandewiede, 1995). Es bleibt dabei offen, wie sich Behandlung im einzelnen vor Ort gestaltet. Rein rechtlich hat ein Gefangener im Rahmen der allgemeinen Fürsorgepflicht für die geistige Gesundheit ein Recht auf Behandlung. Er hat aber keinen Anspruch auf eine bestimmte Interventionsform (Hinrichs, 1995). Bei zeitlich kurzen Haftstrafen sind längerfristige Interventionen nicht umsetzbar und zumeist nicht vorgesehen. Längerfristige therapeutische Angebote haben aufgrund ihrer Kostenintensität Seltenheitscharakter.

Um einen Überblick über die Versorgungs- und aktuelle Forschungspraxis im Rahmen der Straftäterbehandlung zu erhalten, werden im folgenden bekannte (psychologische) Interventionsmaßnahmen vorgestellt. Diese Darstellung soll keineswegs das Bild eines generellen „Behandlungsvollzuges“ deutscher Haftanstalten vermitteln. Die beschriebenen Interventionen stellen nicht die zentralen Maßnahmen der Resozialisierungsbemühungen des Strafvollzuges 
dar, sondern spielen im Vollzugsalltag zumeist eine untergeordnete Rolle. Sie sollen an dieser Stelle aber dennoch Beachtung finden, weil sie im Rahmen der Konzeption der hier vorliegenden Studie im Jugendstrafvollzug wichtige theoretische Impulse und Anstöße zur Umsetzung, Durchführung und Auswertung geliefert haben.

Klassische Psychotherapieverfahren werden in deutschen Strafvollzugsanstalten zumeist in den vom Regelvollzug strukturell und inhaltlich autonomen Sozialtherapeutischen Anstalten bzw. Abteilungen praktiziert. Somit erhält der Großteil aller Inhaftierten keine psychotherapeutische Behandlung. Sie stellt in der Praxis des Strafvollzuges eine Ausnahme dar.

In der Sozialtherapeutischen Anstalt werden therapeutische Maßnahmen sowohl im Einzelwie auch im Gruppensetting durchgeführt. Mit Beginn der Einführung dieser Einrichtungen in den 70er Jahren wurde dort zunächst psychoanalytisch orientiert gearbeitet. Das Behandlungsangebot veränderte sich jedoch hin zu vermehrt humanistischen Therapieverfahren, wobei es die spezifischen therapeutischen Qualifikationen und Kenntnisse der einzelnen Behandler in den Einrichtungen waren, die die konkreten Interventionen bestimmten. Ein Mangel an einem übergeordneten und einheitlichen Behandlungskonzept der Sozialtherapeutischen Anstalt besteht bis heute und „Sozialtherapie“ ist als ein Sammelbegriff für verschiedene Methoden der Verhaltens- und Einstellungsänderung bei Delinquenten mit dem Ziel der Resozialisierung zu verstehen (Egg, 2003). In der Praxis wird die Überweisung eines Straftäters in eine Sozialtherapeutische Anstalt von vollzugsinternen Indikations- und Zulassungsregeln abhängig gemacht. Der Inhaftierte hat keinen Anspruch auf die Behandlung und kann bei mangelndem Erfolg oder mangelndem Wohlverhalten in den Regelvollzug zurückverlegt werden (Basqué, 2003).

Laut Hinrichs (1995) dominiert im Regel- wie im Jugendstrafvollzug das gesprächstherapeutische Vorgehen, im Gesamtkonzept haben die Behandlungsmethoden aber zumeist sozialtherapeutischen Charakter (zu tiefenpsychologisch orientierter Psychotherapie im Justizvollzug siehe auch Pecher, 1999). Ein wesentliches Bestimmungsstück der Therapie stellt die so genannte Tatverarbeitung dar, die in Abschnitt 2.1.2.4 detaillierter besprochen wird.

Ein bekanntes und in der Forschung viel besprochenes kognitiv-orientiertes Interventionsverfahren ist das von Ross und Fabiano (1985) in Kanada entwickelte Reasoning and Rehabilitation Program (R \& R). Es basiert auf dem theoretischen Konzept, antisoziale Denkmuster und Einstellungen wirken im Sinne kognitiver Defizite und stellen einen wesentlichen Risikofaktor für die Entwicklung und Aufrechterhaltung delinquenten Verhaltens dar (vgl. Tabelle 1). Das R \& R- Programm bemüht sich in seiner Konzeption, kriminogenen Faktoren wie 
antisozialer Einstellung, Impulsivität und Substanzmissbrauch zu begegnen. Ein Ziel der Intervention ist es daher, maladaptive Denkmuster zu verändern. Hierfür werden kognitive Fertigkeiten trainiert, die prosoziales Verhalten fördern (Mitchell \& Palmer, 2004).

Das Soziale Training wird häufig im Jugend- aber auch im Erwachsenenvollzug durchgeführt und ist eine den Lerntheorien entlehnte Behandlungskonzeption. Durch die Vermittlung spezifischer sozialer Fähigkeiten sollen spezielle Defizite kompensiert und die soziale Kompetenz insgesamt verbessert werden (Basqué, 2003). Der Alltag des Inhaftierten in seiner Wohngruppe wird dabei als zentrales therapeutisches Lernfeld verstanden. Maßnahmen wie entlassungsvorbereitende Lockerungen (Ausgang, Urlaub oder Freigang) werden als konkrete Trainingsfelder begriffen. Deutschlandweit existieren verschiedene Konzeptualisierungen des Sozialen Trainings, wie etwa das „Tegeler Modell“ von Kremer (1977), das mit dem Anspruch verknüpft ist, auf das Gesamtsystem des Strafvollzuges Einfluss zu nehmen und dem Häftling von der Aufnahme bis zur Entlassung eine ,strukturierte Trainingskette“ vorzugeben (Otto, 2003 S. 116). Hierbei sind alle Fachdienste wie auch Laientrainer beteiligt, um vollzugliche und therapeutische Funktionen zu integrieren. Soziale Trainingsmaßnahmen haben den Vorteil, dass sie für alle Vollzugsformen 
Tabelle 1

(Intramurale) Behandlungsmethoden im Überblick

\begin{tabular}{|c|c|c|c|c|c|c|c|}
\hline Intervention & Zielgruppe & $\begin{array}{l}\text { Theoretische } \\
\text { Grundlage }\end{array}$ & Inhalte/Module & Länge & Setting & $\begin{array}{l}\text { Behandlungs- } \\
\text { ziele }\end{array}$ & $\begin{array}{l}\text { Wirksamkeit } \\
\text { (Rückfälligkeit) /Quelle }\end{array}$ \\
\hline $\begin{array}{l}\text { Reasoning and } \\
\text { Rehabilitation } \\
\text { Program } \\
\text { Ross \& Fabiano } \\
1985\end{array}$ & $\begin{array}{l}\text { Jugendliche und } \\
\text { erwachsene Inhaf- } \\
\text { tierte }\end{array}$ & $\begin{array}{l}\text { kognitiv- } \\
\text { behaviorale/soziale } \\
\text { Lerntheorien }\end{array}$ & $\begin{array}{l}\text { Problemlösetraining } \\
\text { Training soz. Fertigkeiten } \\
\text { Umgang m. Emotionen } \\
\text { Werte/Normorientierung } \\
\text { Kreatives/kritisches Den- } \\
\text { ken }\end{array}$ & ca. $70 \mathrm{~h}$ & $\begin{array}{l}\text { Gruppe } \\
(6-12 \\
\text { Klienten })\end{array}$ & $\begin{array}{l}\text { Reduktion von } \\
\text { Rückfälligkeit } \\
\text { nach Haftentlas- } \\
\text { sung }\end{array}$ & $\begin{array}{l}\text { Raten erneuter Verurtei- } \\
\text { lung/Inhaftierung } \\
\text { reduziert } \\
\text { Robinson, } 1995 \\
\text { Robinson \& Porporino, } 2001 \\
\text { Friendship et al., } 2003 \\
\text { Mitchell \& Palmer, } 2004 \\
\end{array}$ \\
\hline $\begin{array}{l}\text { Multisystemische } \\
\text { Therapie } \\
\text { Henggeler \& Bor- } \\
\text { duin, } 1992\end{array}$ & $\begin{array}{l}\text { Delinquente Jugen- } \\
\text { dliche }\end{array}$ & $\begin{array}{l}\text { familien- und um- } \\
\text { weltbasiert }\end{array}$ & $\begin{array}{l}\text { Coaching der Eltern im } \\
\text { Erziehungsverhalten }\end{array}$ & $\begin{array}{l}\text { 3-5 } \\
\text { Monate }\end{array}$ & vor Ort & $\begin{array}{l}\text { Förderung pro- } \\
\text { sozialen Verhal- } \\
\text { tens/ Rückfall }\end{array}$ & $\begin{array}{l}\text { Effekt } d=.55 \\
\text { nach Curtis et al., } 2004\end{array}$ \\
\hline Sozialtherapie & $\begin{array}{l}\text { Erwachsene und } \\
\text { jugendliche Inhaf- } \\
\text { tierte }\end{array}$ & $\begin{array}{l}\text { Sozialtherapeutisch } \\
\text { (psychotherapeutisch/ } \\
\text { pädagogisch) }\end{array}$ & $\begin{array}{l}\text { „therapeutisches Milieu“ } \\
\text { Problemlösetrainings }\end{array}$ & $\begin{array}{l}6-24 \\
\text { Monate }\end{array}$ & $\begin{array}{l}\text { Gruppe } \\
\text { u. } \\
\text { Einzel }\end{array}$ & Legalbewährung & $\begin{array}{l}\text { Effekt bei } r=.11^{*} \\
\text { Lösel, } 2003 \\
\text { Ortmann, } 1994 \\
\text { Waxweiler, } 1980\end{array}$ \\
\hline $\begin{array}{l}\text { Anti- } \\
\text { Aggressivitäts- } \\
\text { Trainings } \\
\text { u.a. Weidner et al., } \\
1987\end{array}$ & $\begin{array}{l}\text { Jugendliche u. er- } \\
\text { wachsene Gewalt- } \\
\text { u. Sexualstraftäter }\end{array}$ & $\begin{array}{l}\text { sozialpädagogisch/ } \\
\text { kognitiv- verhal- } \\
\text { tensthera- } \\
\text { peutisch }\end{array}$ & $\begin{array}{l}\text { konfrontativ „heißer } \\
\text { Stuhl“; Rollenspiel } \\
\text { Selbstinstruktion } \\
\text { Entspannungsmethoden }\end{array}$ & $\begin{array}{l}4-10 \\
\text { Monate }\end{array}$ & $\begin{array}{l}\text { Gruppe } \\
(\max .12)\end{array}$ & $\begin{array}{l}\text { Abnahme allg. } \\
\text { Aggression, } \\
\text { reaktiver Aggres- } \\
\text { sivität }\end{array}$ & $\begin{array}{l}\text { Verminderte Rückfallintensität } \\
\text { Dünkel \& Drenkhahn, } 2001 \\
\text { Ohlemacher et al., } 2000\end{array}$ \\
\hline $\begin{array}{l}\text { Soziale Trainings } \\
\text { u.a. Otto, } 1986\end{array}$ & $\begin{array}{l}\text { Jugendliche und } \\
\text { erwachsene Inhaf- } \\
\text { tierte }\end{array}$ & lerntheoretisch & $\begin{array}{l}\text { Rollenspiel } \\
\text { Informationsvermittlung }\end{array}$ & $\begin{array}{l}2-12 \\
\text { Monate }\end{array}$ & $\begin{array}{l}\text { Gruppe } \\
(\max .9)\end{array}$ & $\begin{array}{l}\text { Verbesserung } \\
\text { sozialer Kompe- } \\
\text { tenzen }\end{array}$ & \\
\hline Drogentherapie & $\begin{array}{l}\text { Jugendliche und } \\
\text { erwachsene Inhaf- } \\
\text { tierte mit Drogen- } \\
\text { problematik }\end{array}$ & $\begin{array}{l}\text { verhaltens- } \\
\text { therapeutisch/ } \\
\text { medizinisch }\end{array}$ & $\begin{array}{l}\text { (Substitution) } \\
\text { Psychotherapie } \\
\text { Psychoedukation } \\
\text { Entspannungsmethoden }\end{array}$ & & $\begin{array}{l}\text { Gruppe } \\
\text { u. } \\
\text { Einzel }\end{array}$ & $\begin{array}{l}\text { Abstinenz } \\
\text { Selbstkontrolle } \\
\text { Infektionspro- } \\
\text { phylaxe }\end{array}$ & \\
\hline $\begin{array}{l}\text { Schulisch- } \\
\text { berufliche } \\
\text { Förderung** }\end{array}$ & $\begin{array}{l}\text { Jugendliche und } \\
\text { erwachsene } \\
\text { Inhaftierte }\end{array}$ & & $\begin{array}{l}\text { Erwerb schulischer und } \\
\text { beruflicher Qualifikation, } \\
\text { wie Hauptschulabschluss, } \\
\text { Lehrberuf, Fernstudium } \\
\text { etc. }\end{array}$ & $\begin{array}{l}\text { 6-36 } \\
\text { Monate }\end{array}$ & & $\begin{array}{l}\text { Verbesserung } \\
\text { der } \\
\text { Resozialisierung }\end{array}$ & $\begin{array}{l}\text { Kaum präventive Wirkung } \\
\text { Geissler, } 1991 \\
r=.11 \text { für Schulausbildungs- } \\
\text { programme } \\
\text { Pearson \& Lipton, } 1999\end{array}$ \\
\hline
\end{tabular}

Anmerkungen. Die dargestellten Interventionen stellen eine Auswahl verschiedener Maßnahmen dar, die sich nach Literatursichtung als die am meisten diskutierten herausstellten.

* Ergebnis der Evaluation ausschließlich deutscher Sozialtherapeutischer Anstalten.

** Hierbei handelt es sich zwar nicht um eine „Behandlungsmaßnahme“ im engeren Sinne, sie stellt aber eine zentrale resozialisierende Maßnahme dar 
geeignet sind, keine langjährige psychotherapeutische Ausbildung voraussetzen und leicht zu installieren sind (Basqué, 2003).

Neben intramuralen Behandlungsmöglichkeiten Straffälliger sei der Vollständigkeit halber an dieser Stelle ein ambulantes Interventionsverfahren zur Verminderung der Rückfälligkeit Jugendlicher erwähnt. Die so genannte Multisystemische Therapie (MST) ist ein evidenzbasiertes familien-fokussiertes Interventionsverfahren, das vor allem in den USA bei Störungen des Sozialverhaltens im Jugendalter zum Einsatz kommt. Die jugendlichen Klienten sind hauptsächlich wegen Drogengebrauchs und/oder Gewalttätigkeit mit dem Gesetz in Konflikt geraten, haben bereits eine Fremdunterbringung hinter sich oder ihnen droht eine solche. Sie entstammen vorwiegend „Multiproblemfamilien“, die durch multiple Belastungsfaktoren wie z.B. Arbeitslosigkeit, Armut, Drogenabhängigkeit der Eltern gekennzeichnet sind (Heekerens, 2006). Die Interventionskomponenten des MST entstammen im Kern der Tradition der Sozialarbeit. Die Behandlung findet vor Ort in Form von Hausbesuchen statt und bemüht sich zugleich um die Aktivierung von Ressourcen des Gemeinwesen. In verschiedenen Studien konnte gezeigt werden, dass delinquente Jugendliche, die mittels MST behandelt wurden, eine geringere Wiederverurteilungs- und Inhaftierungsrate aufwiesen als die jeweilige Kontrollgruppe (Curtis, Ronan \& Borduin, 2004; Timmons-Mitchell, Bender, Kishna \& Michtell, 2006).

Neben globalen Behandlungsmaßnahmen bestehen im Strafvollzug in jüngerer Zeit vermehrt spezifische Behandlungsprogramme. So richten sich Anti-Aggressivitäts-Trainings (AAT) deliktspezifisch an solche Inhaftierte, die wegen Gewalt- und/oder Sexualstraftaten verurteilt worden sind. Das ursprüngliche Konzept des AAT wurde Mitte der 80er Jahre entwickelt (Weidner, 1990). Seitdem sind eine Reihe von weiterentwickelten Maßnahmen entstanden, die das Behandlungsziel haben, die Gewaltbereitschaft, hauptsächlich jugendlicher Inhaftierter, zu reduzieren, das Verantwortungsbewusstsein zu steigern und die Teilnehmer angemessene Konfliktlösestrategien erlernen zu lassen (Bosold, Prasse \& Lauterbach, 2006). Im Ursprungskonzept wie auch in vielen weiterentwickelten Programmen stellt der so genannte „Heiße Stuhl“ als Konfrontationstechnik einen therapeutischen Kernbestandteil dar. Die Trainingsgruppe besteht aus etwa sechs bis acht Teilnehmern. Die Behandlungsdauer erstreckt sich durchschnittlich über ein halbes Jahr mit 15 bis 30 mehrstündigen wöchentlichen Sitzungseinheiten. Das Behandlungsteam besteht meistens aus Psychologen, Sozialpädagogen, Bediensteten des Allgemeinen Vollzugsdienst und ehrenamtlichen Mitarbeitern. 
In Bezug auf drogentherapeutische Maßnahmen ist zunächst zu berücksichtigen, dass sich die Anzahl der Drogenkonsumenten in den letzten Jahrzehnten von einer Minderheit zu einer zentralen Gruppe in der Population des Strafvollzuges entwickelt hat (Obrist \& Werdenich, 2004). Wie bereits in Abschnitt 2.1.1.1 dargestellt, erfüllen viele der Inhaftierten, die vor ihrer Haft regelmäßig Alkohol und/oder Drogen konsumiert haben, die Kriterien einer Missbrauchs- oder Abhängigkeitsdiagnose (bei jugendlichen Häftlingen liegen die Prävalenzraten zwischen 40 und 50\%). Der Behandlung, Betreuung und Versorgung von Drogenabhängigen kommt im Strafvollzug daher eine besondere Bedeutung zu, wobei der Behandlungsbedarf zumeist größer ist als das Angebot (Stöver, 1999).

Behandlungsangebote sind sowohl am Ausstieg aus der Sucht wie auch an sozialer Stabilisierung des Inhaftierten orientiert. Sie sind meistens „freiwillig“ und nicht angeordnete Zwangsmaßnahmen, wobei in vielen Fällen eine Behandlung mit Vergünstigungen wie Lockerungen verbunden ist. Neben dem körperlichem Entzug mittels Entgiftung existieren seit langem abstinenzorientierte Einzel- und Gruppenpsychotherapien, die kurz-, mittel- oder langfristig angeboten werden. Mit dem vermehrten Auftauchen von HIV und Hepatitis $\mathrm{C}$ bei drogenabhängigen Inhaftierten rückte die Infektionsprophylaxe verstärkt in den Vordergrund der Behandlung. So gibt es mittlerweile einige Substitutions- und Spritzentauschprogramme in Gefängnissen, wobei die Substitution mit Methadon die häufigste und am wenigsten umstrittenste Methode darstellt. So genannte „niedrigschwellige“ Versorgungsangebote mit der Orientierung der „harm reduction“ finden in Haftanstalten noch geringe Akzeptanz. Im Rahmen solcher Angebote wird das Suchtverhalten von Abhängigen, die nicht abstinent sein können oder wollen, dahingehend unterstützt, dass deren „Gesundheitsrisiko sinkt und die Überlebenschance steigt“" (Stöver, 1999, S. 43; zu Substitutions- und Nadeltauschprogrammen in Gefängnissen ausführlicher bei Stöver, 2002).

Verurteilte drogenabhängige Straftäter haben überdies die Möglichkeit, sich über Behandlungsauflagen auch außerhalb des Strafvollzuges im Rahmen so genannter „Therapie statt Strafe“-Programme stationär behandeln zu lassen (Obrist \& Werdenich, 2004, S. 44).

Nicht zuletzt kommen besonders im Jugendvollzug Maßnahmen schulisch-beruflicher Förderung eine zentrale resozialisierende und rehabilitierende Bedeutung $\mathrm{zu}$. Im erzieherischen Setting werden bestenfalls im gesamten Haftalltag Lernangebote und -hilfen bereit gestellt. Bildungsabschlüsse und berufliche Bildungsförderung haben das Ziel, die soziale Integration zu erleichtern. Dem (jugendlichen) Inhaftierten wird die Möglichkeit gegeben, schulische Defizite auszugleichen, kontinuierlich und regelmäßig zu arbeiten, Lebensbewältigung zu 
erlernen, seinen Alltag zu strukturieren und zu lernen, mit Stress umzugehen. Schulpflichtige Inhaftierte erhalten Schulunterricht in Anlehnung an die für öffentliche Schulen geltenden Vorschriften, an Haupt- und Sonderschulunterricht können jedoch auch nicht-schulpflichtige Gefangene teilnehmen. Zudem ist bei beruflicher Aus- und Fortbildung berufsbildender Unterricht vorgesehen.

Die schulisch-berufliche Ausbildung innerhalb und außerhalb der Strafanstalt hat neben dem generellen Ziel des Erwerbs von Wissen und Kompetenzen die Aufgabe, Inhaftierte in dem Bereich der Persönlichkeitsbildung, ihrem Selbstvertrauen und der Eigenverantwortlichkeit zu fördern (Walkenhorst, 2002).

\subsubsection{Aufarbeitung der Straftat}

Im Rahmen der Behandlung von (inhaftierten) Straftätern spielt die Tatverarbeitung oder auch Tataufarbeitung grundsätzlich eine zentrale Rolle - unabhängig davon, welche spezielle Intervention gewählt wird. Zur Aufarbeitung der Straftat kommt es somit im Einzel- wie auch im Gruppensetting.

Kommt in der Straftat die psychopathologische Problematik eines Straftäters zum Ausdruck, ergibt sich auf nahe liegende Weise die Notwendigkeit zur therapeutischen Auseinandersetzung mit dem Geschehenen. Aber auch bei psychisch gesunden Delinquenten, die eine Strafe verbüßen, ist die Bearbeitung der Straftat von diagnostischem und prognostischem Wert. Zeigt der Inhaftierte Einsicht in die Unrechtmäßigkeit seiner Taten, zeigt er Reue und berücksichtigt er seine Verhaltensanteile am Zustandekommen der Straftat, wird dies zumindest wegweisend für eine positive Legalprognose gewertet (Hinrichs, 1994). Dahinter steht die Annahme, dass ein Täter, der sich sein Handeln motivisch, situativ und persönlich gar nicht erklären kann, wahrscheinlich auch stärker rückfallgefährdet ist (Hinrichs, 1993). Eine sich verändernde Repräsentanz der Straftat(-en) ermöglicht es einem Behandler außerdem, mögliche Entwicklungen auf Seiten des Klienten zu erkennen. Die Erarbeitung der Tatphänomenologie (Tatvorlaufzeit, unmittelbares Tatgeschehen, Nachtatphase) mit ihren möglicherweise für den Straftäter typischen Mustern im Deliktablauf geschieht auf den Ebenen der Kognitionen, des Affektes, der Motivation und sozialer Aspekte. Bei der Deliktbearbeitung wird antisoziales Denken als ein wesentlicher Risikofaktor für (fortgesetztes) delinquentes Verhalten gesehen und daher gezielt modifiziert. Hierbei wird von Seiten des Therapeuten darauf geachtet, dass der Klient Neutralisierungstendenzen, wie Bagatellisierung, Leugnen von Verantwortlichkeit und der Schädigung des Opfers, Verharmlosung oder Rationalisieren verringert (vgl. auch Sykes \& Matza, 1957). 
Ist das Deliktszenarium ausgearbeitet und mögliche Risikofaktoren sowie potentielle Schutzfaktoren identifiziert, werden im Rahmen einer Kriminaltherapie interne und externe Kontrollmechanismen und Handlungsalternativen erarbeitet.

Kritisch angemerkt wird in diesem Zusammenhang, dass das Konstrukt der Tataufarbeitung (bisher) keine empirisch fundierte Grundlage und keine allgemein verbindliche therapeutische Vorgehensweise aufweisen kann, so dass die Analyse und Bewertung des Prozesses durch die persönliche Herangehensweise des Behandlers gekennzeichnet ist (Simons, 1996). Einer allzu optimistischen Einschätzung der Tataufarbeitung als ein zuverlässiges diagnostisches und prognostisches Instrumentarium ist daher kritisch zu begegnen. Nicht zuletzt auch deshalb, weil es in diesem Prozess um eine Auseinandersetzung mit häufig schambesetzten Gedanken und Gefühlen geht, Einschränkungen durch Effekte sozialer Erwünschtheit, Probleme des Selbstwertes und des Erinnerbaren gegeben seien können, die einen Rückschluss auf die Persönlichkeitsentwicklung und die Legalprognose eines Inhaftierten stark beeinträchtigen.

\subsubsection{Wirksamkeit intramuraler Interventionsmaßnahmen}

Straftäterbehandlung ist strafrechtlich als ein Teil der Spezialprävention zu verstehen, die alle Einwirkungen auf den Straftäter mit dem Ziel der Rückfallverhinderung umfasst. Zur Spezialprävention gehört neben der Behandlung auch die Individualabschreckung und die Sicherung (Dölling, 2000). Die Frage, ob Straftäterbehandlung hinsichtlich der Reduktion von Rückfällen effektiv ist oder nicht, stellt sich heute in dieser isolierten Betrachtungsweise nicht mehr. Es geht vielmehr gegenwärtig um die spezifischere Frage: „What works how with whom under which conditions ?" (Steller, 2003, S. 10).

Metaanalytische Befunde von Behandlungsprogrammen jugendlicher und heranwachsender Straftäter kommen hauptsächlich aus dem nordamerikanischen Raum. Obwohl die dortigen Programme, Zielgruppen, Effektkriterien und Behandlungskontexte sehr heterogen sind und zum Beispiel auch Früh-Interventionen bei dissozialen Kindern häufig mit eingeschlossen worden sind, so lassen sich konsistente Behandlungseffekte nachweisen. Eine generelle Effektstärke von $r=.10$ wird als realistische Schätzung angegeben (Lösel, 1996). Dies entspricht einer Reduzierung der Rückfallquote um ca. 10\%. Ähnliche Effekte finden sich mit $r=.12$ auch im Bereich der (deutschen) Sozialtherapie erwachsener Inhaftierter (Egg, Pearson, Cleland \& Lipton, 2001). Im Rahmen einer umfangreichen Metaanalyse zur Behandlung delinquenter Jugendlicher von Lipsey (1992) konnte in über 60\% der 449 berücksichtigten Primärstudien eine signifikante Differenz zugunsten der Behandlung nachgewiesen werden. Diesen 
Befunden wird oft mit dem Argument begegnet, dass die Effektstärken aus anderen Bereichen psychotherapeutischer Interventionen deutlich höher liegen. Die Delinquenzbehandlung scheint sich in der Praxis gerade bei dissozialen Störungen als besonders schwierig darzustellen. Faktoren wie die Therapiemotivation, das Milieu, das spezielle Setting, die angelegten Erfolgskriterien (zumeist Rückfälligkeit) und die teilweise längeren Follow-up-Zeiträume in der Behandlung delinquenter Patienten reduzieren vermutlich die Wahrscheinlichkeit eines Nachweises der möglichen Effektivität der Intervention. In der Überprüfung differenzieller Effekte zeigt sich, dass unterschiedliche Effektstärken mitunter auf verschiedene inhaltliche Aspekte der Behandlung zurück zu führen sind. „Sowohl innerhalb wie auch außerhalb des Jugendstrafrechts sind stärker strukturierte, kognitive und verhaltensorientierte sowie multimodale Interventionen, die auf konkrete Fertigkeiten abzielen, wirksamer als z.B. nondirektive Beratung, Gesprächsgruppen oder unstrukturierte Fallarbeit“ (Lösel, 2003 S. 24).

In einer weiteren Studie zur Überprüfung der Wirksamkeit intramuraler Behandlung jugendlicher und erwachsener Straftäter von Andrews, Zinger, Hoge, Bonta, Gendreau und Cullen (1990), die 154 Einzelstudien einschließt, kommen die Autoren zu dem Ergebnis, dass sich drei Prinzipien angemessener Behandlung formulieren lassen, die bis heute als verbindlich betrachtet werden können. Nach dem risk principle soll im Rahmen jeglicher Straftäterbehandlung eine angemessene Behandlungsebene ausgewählt werden, d.h. Probanden mit hohem (Rückfall-) Risiko benötigen intensive Maßnahmen (,high-level service“). Bei Probanden mit ohnehin geringem Risiko sollten hingegen keine hohen Behandlungseffekte im Vergleich zu einer Kontrollgruppe erwartet werden (Lösel, 2003, S. 24). Das need principle beschreibt den Anspruch, dass Behandlung sich auf die empirisch nachweislichen kriminogenen Faktoren konzentrieren soll. Dazu gehören z.B. die Veränderung antisozialer Einstellungen und Peer-Kontakte, die Stärkung der Selbstkontrolle, die Vermittlung sozialer Fertigkeiten, die Reduktion von Drogenabhängigkeit oder die Verbesserung der Chancen auf dem Arbeitsmarkt. Andrews und seine Kollegen (1990) konnten zeigen, dass Maßnahmen zur Veränderung des Selbstbildes, der Minderung von Ängsten und unspezifischen Persönlichkeitsproblemen kriminaltherapeutisch weniger erfolgsversprechend $\mathrm{zu}$ sein scheinen. Das dritte Prinzip, das so genannte responsitivity principle betrifft schließlich die speziellen Merkmale erfolgreicher Delinquenzbehandlung, die die Ansprechbarkeit der Straftäter berücksichtigen und sich an ihre Lernkompetenzen anpassen. Dazu gehören Maßnahmen wie Rollenspiele, Verstärkung, Prompting oder kognitive Umstrukturierung, die sich zumeist auf die Vermittlung konkreter Fertigkeiten beziehen. Unstrukturierte Gruppenaktivitäten, Abschreckungsme- 
thoden und nondirektive Ansätze scheinen in der Rückfallprävention generell weniger erfolgreich zu sein (Andrews \& Bonta, 2004).

Berücksichtigen Interventionsmaßnahmen diese drei Prinzipien und stellen somit ,angemessene Maßnahmen“ dar, ergeben sich Effektstärken um Phi=.29 für die intramurale Behandlung Jugendlicher und um Phi=.34 für erwachsene Inhaftierte (Andrews et al., 1990). Gemeindenahe Maßnahmen zeigen eine tendenzielle Überlegenheit gegenüber der Behandlung in Anstalten wo z.B. mit gegenläufig wirksamen Prisonisierungseffekten (negative Wirkweisen der Anpassungsprozesse an interne subkulturelle Normen und Werte) gerechnet werden muss. Bezüglich der verschiedenen Effektkriterien ist zu berücksichtigen, dass Rückfalldaten im allgemeinen niedrigere Effekte erbringen als „weichere“ Maße wie etwa die Veränderung der Einstellung (Lösel, 2003). Darüber hinaus konnte gezeigt werden, dass unangemessene oder schlecht implementierte Behandlungsprogramme sogar negative Auswirkungen haben und die Rückfallquoten im Vergleich zur unbehandelten Kontrollgruppe erhöhen können (Dünkel \& Drenkhahn, 2001; Andrews et al., 1990).

Andere metaanalytische Befunde bestätigen die von Lipsey und Andrews et al. nachgewiesene differentielle Wirksamkeit verschiedener Behandlungsmaßnahmen. Garrett (1985) sowie Gottschalk, Davidson, Gensheimer und Mayer (1987) berichten, dass behaviorale Programme in der Straftäterbehandlung besser abschneiden als andere. Izzo und Ross (1990) finden im Vergleich stärkere Effekte kognitiver Behandlungsprogramme und Antanowicz und Ross (1993) liefern Befunde dafür, dass kognitiv-behaviorale sowie multi-modale Programme die höchste rückfallpräventive Wirkung besitzen. Ein Beispiel für eine solche Intervention ist das sozial-kognitive Skill-Training von Robert Ross (siehe auch Reasoning and Rehabilitation Program Abschnitt 2.1.2.3; z.B. Ross, Fabiano \& Ross, 1986).

$\mathrm{Zu}$ den genannten Prinzipien erfolgreicher Straftätertherapie ergänzt Lösel (1993) die Relevanz eines positiven institutionellen Klimas, das sich beispielsweise in einem sensiblen, konstruktiven und unterstützenden Umgang des Personals, das sorgfältig ausgewählt, geschult und supervidiert werden sollte, ausdrückt, und die Neutralisierung ungünstiger sozialer Netzwerke innerhalb und außerhalb der Haft. Ferner spielt die Stützung protektiver Faktoren des sozialen Umfelds des Gefangenen (z.B. positive Partnerbeziehung, differentielle Nachsorge, Rückfallprävention) eine zentrale Bedeutung in der Behandlung (Dünkel \& Drenkhahn, 2001).

Die Forschungsergebnisse lassen es jedoch bisher nicht zu, in einen grundsätzlichen Behandlungsoptimismus zu verfallen. Es besteht derzeit noch ein erheblicher Mangel an kontrollier- 
ten Studien im Bereich der (intramuralen) Straftäterbehandlung. Lange Zeit waren zahlreiche Behandlungsprogramme theoretisch sowie störungsspezifisch nicht oder zu wenig fundiert. Die bisherigen Ergebnisse zeigen, dass Maßnahmen z.B. zur Förderung der Selbstkontrolle, welche an Merkmalen ansetzen, die auch in der Quer- und Längsschnittforschung als relativ gut belegte Prädiktoren persistierender Straffälligkeit gelten z.B. kognitive Defizite, Impulsivitätprobleme, kriminogene Denkmuster, erfolgreich sein können. Umfassende theoriengeleitete Evaluationen stehen bisher in der Straftäterbehandlung noch am Anfang, auch deshalb, weil Behandlungskonzepte oft ungenügend implementiert werden und Behandlungsmerkmale undefiniert bleiben (Lösel, 2003). Die Qualitätssicherung von Behandlungsprogrammen im Strafvollzug ist als eine im Rahmen der Verbesserung des Resozialisierungsangebotes zentrale Herausforderung zu betrachten und bleibt in der forensischen Forschung und Praxis angesichts der bestehenden Sozialisations- und Bildungsdefizite der hoch selektierten Population des Jugend- und Erwachsenenstrafvollzuges relevantes Ziel.

Um theoriegeleitete Interventionsstrategien für die Behandlung jugendlicher und heranwachsender Inhaftierter zu entwickeln, bietet es sich an, Ansätze aus bereits existierenden Forschungsbereichen zu übernehmen und für das spezifische Setting des Strafvollzuges zu adaptieren und somit nutzbar zu machen. Die im folgenden vorgestellte Methode des Expressiven Schreibens kann als ein niedrigschwelliges und ökonomisches Angebot für Inhaftierte mit kurzen Haftstrafen und vergleichsweise hoher psychosozialer Belastung aufgefasst werden. Es bietet u.a. die Möglichkeit, sich mit schambesetzten Erfahrungen sowie kriminogenen Denkmustern bereits zu Beginn der Haftzeit schriftlich auseinanderzusetzen. Es ermöglicht darüber hinaus, den Ansatz der Tataufarbeitung aufzugreifen und schriftlich durchzuführen. Bevor auf die speziellen Überlegungen, die es für die Durchführung einer Schreibintervention im Jugendstrafvollzug zu berücksichtigen gilt, näher eingegangen wird, werden zunächst die Methode des Expressiven Schreibens, ihre Entwicklung, die Anwendungsbereiche und die verschiedenen Studien zur Wirksamkeit dargestellt.

\subsection{Expressives Schreiben: Stand der Forschung}

\subsubsection{Das Schreibparadigma und seine Anwendungen}

Das Erleben belastender Lebensereignisse oder Traumata fordert vom Individuum eine Anpassungsleistung an eine veränderte Lebenssituation (vgl. Traue, 1998). Ähnlich wie im Sinne des Life-Event-Ansatzes (Holmes \& Rahe, 1967) geht auch der Sozialpsychologe J.W. 
Pennebaker davon aus, dass belastende Lebensereignisse wie etwa der Tod einer nahe stehenden Person, Scheidung, Eintritt ins College oder Arbeitslosigkeit sich negativ auf die physische und psychische Gesundheit auswirken (Pennebaker, 2002). Ein zentraler Aspekt jeglicher therapeutischer Intervention besteht laut Pennebaker in der Möglichkeit, eigene (traumatische) Erfahrungen in Worte zu übersetzen oder, anders ausgedrückt, in der Selbstöffnung oder Selbstoffenbarung - engl. „self-disclosure“. Die Grundannahme im Rahmen der Theorie der Inhibition und Konfrontation von Pennebaker $(1988 ; 1989)$ besteht daher auch in dem natürlichen menschlichen Bedürfnis, nach einem belastenden oder traumatischen Erlebnis mit anderen darüber zu sprechen und dabei den eigenen Gefühlen Ausdruck zu verleihen (vgl. 2.2.2.1). Aus Scham oder Angst vor negativen Reaktionen werde diesem Bedürfnis nach Selbstoffenbarung oft nicht nachgegangen. Die Inhibition von Gefühlen und Gedanken kann langfristig als niedrigschwelliger Stressor wirken, der das ANS aktiviert und das Immunsystem schwächen kann, was wiederum die Vulnerabilität für körperliche und physische Beschwerden erhöht. Eine ideale Möglichkeit, sich trotz möglicher Scham- oder Angstgefühle mit belastenden Erfahrungen auseinander zu setzen, liegt für Pennebaker im Expressiven Schreiben (ES). Es bietet die Möglichkeit, sich mitzuteilen und begünstigt gleichzeitig eine konfrontative und konstruktive Verarbeitung des Erlebnisses.

Im Rahmen der ersten Studie Pennebakers zu diesem Thema wurden Studierende gebeten, an vier Tagen jeweils 15 Minuten lang entweder über die traumatischste Erfahrung ihres Lebens oder über ein banales Thema zu schreiben (Pennebaker \& Beal, 1986). Ein halbes Jahr nach der Untersuchung konnte beobachtet werden, dass die Studierenden, die über ihre Gedanken und Gefühle zu einem traumatischen Ereignis geschrieben hatten, im Vergleich zur Kontrollgruppe und der Gruppe, die rein faktisch ohne eigene Emotionen auszudrücken über ihr Trauma schrieb, seltener das Gesundheitszentrum der Universität aufgesucht hatten. Dieses Ergebnis und der Umstand, dass es sich beim Expressiven Schreiben um eine einfach handhabbare, kostengünstige, flexibel einsetzbare und ressourcensparende Intervention handelt, löste in der Folgezeit rege Forschungsaktivität aus, so dass bis zum Jahr 2006 über 250 Studien publiziert wurden, die das experimentelle disclosure-Design in verschiedenen Variationen verwendeten (vgl. Frattarolli, 2006).

Bei einer typischen Schreibstudie gibt es meist zwei Gruppen (Schreib- und Kontrollgruppe), denen die Probanden randomisiert zugeteilt werden. Die Kontrollgruppe wird zumeist instruiert, über ein neutrales Thema ohne jeglichen Ausdruck von Emotionen oder Gedanken zu schreiben (z.B. Tagesablauf). Es wird an drei bis vier Tagen für jeweils 15 bis 20 Minuten in einem dafür vorgesehenen Laborraum in privater und ungestörter Atmosphäre geschrieben. 
Die verfassten Essays werden anonymisiert, Rückmeldung wird nicht gegeben. In der Originalinstruktion zum ES wird der Proband gebeten, seine tiefsten Gedanken und Gefühle bezüglich der bisher traumatischsten Erfahrung seines Lebens zu erkunden und niederzuschreiben. In den verschiedenen Studien wurden sowohl kurzfristige wie langfristige Konsequenzen des Schreibens auf das Befinden und die Gesundheit der Probanden untersucht (vgl. 2.2.3).

In den mit unterschiedlichen Populationen durchgeführten Studien zum ES zeigen sich stabil hohe Anteile von Berichten über traumatisch zu bezeichnende Erlebnisse (Lepore \& Smyth, 2002). Auch in den zunächst überwiegend untersuchten mittelsschichtigen Stichproben wählen die Probanden zumeist Lebensereignisse wie Todesfälle von nahe stehenden Personen, Scheidung, schwere Unfälle, Missbrauchserfahrungen usw. In manchen Studien wurden die Schreibthemen aber auch vorgegeben, wie z.B. Arbeitslosigkeit (Spera, Buhrfeind \& Pennebaker, 1994), Probleme bei Studienbeginn (Cameron \& Nicholls, 1998; Pennebaker \& Francis, 1996;) oder das eigene negative Körperbild (Earnhardt, Martz, Ballard \& Curtin, 2002). Zwischen dem Schweregrad des traumatischen Erlebnisses und der Wirkung des Schreibens ließen sich bisher keine Zusammenhänge finden (Sloan \& Marx, 2004b). In den unterschiedlichen Schreibinstruktionen finden sich auch solche, die den Probanden auffordern, über positive Lebensereignisse (Abele, 1990; Burton \& King, 2004; Sharsky, 1997) oder eigene Lebensziele (King, 2001) zu reflektieren. Um eine konstruktive Neubewertung des Erlebten in Gang zu setzen, wurde die Schreibinstruktion in verschiedenen Studien variiert und über die Schreibtage strukturiert vorgegeben. So wiesen Cameron und Nicholls (1996) ihre Probanden beispielsweise an, jeweils am Ende der Schreibsitzung, Vorschläge zum Coping und somit zum konstruktiven Umgang mit den Erlebnissen aufzulisten. Um ihre Selbstregulationsfähigkeit zu fördern, wurden die Probanden angeleitet, die eigenen Coping-Strategien schließlich $\mathrm{zu}$ bewerten und gegebenenfalls zu revidieren. In einer weiteren Variante der angeleiteten Selbstöffnung, des so genannten Helpings, sollten sich Probanden in die Rolle eines Beraters versetzen und einer anderen hypothetischen Person, die das Gleiche erlebt hat, schriftlich hilfreiche Empfehlungen geben (Kröner-Herwig, Linkemann \& Morris, 2004). Auch Gidron und Kollegen (2002) leiteten ihre Probanden am letzten Schreibtag dabei an, ihren künftigen Umgang mit dem belastenden Ereignis zu beschreiben.

In der Mehrzahl der Studien erfolgte die Konfrontation mit dem belastenden Erlebnis schriftlich. Es wurden jedoch auch Untersuchungen zu den Auswirkungen mündlicher (Pennebaker, 1999; Murray \& Segal 1994) oder internetbasierter (Lange, van de Ven \& Schrieken, 2003; Sheese, Brown \& Graziano, 2004) Selbstöffnung durchgeführt. Die vielfältige Anwendung 
des Paradigmas zeigt sich vor allem bezüglich der unterschiedlichen Populationen, mit denen die Intervention bisher durchgeführt wurde. So wurden bereits Probanden mit verschiedenen körperlichen Beeinträchtigungen wie z.B. Asthma (Harris, Thoresen \& Faul, 2004; Harris, Thoresen, Humphreys, \& Faul, 2005), Rheuma (Kelley, Lumley \& Leysen, 1998), HIVpositive Patienten (Mann, 2001), Krebspatienten (Stanton, Danoff-Burg, Sworowski et al., 2002; Walker, Nail \& Croyle 1999; Zakowski, Ramati, Morton, Johnson \& Flanigan, 2004) oder Probanden mit psychischen Beeinträchtigungen wie z.B. emotionale Störungen bei Jugendlichen (Evans, 2000), Patienten mit affektiven Störungen (Forston, 1992) und Patienten mit Posttraumatischer Belastungsstörung (Gidron, Peri, Connelly \& Shalev, 1996) untersucht. Einen ähnlichen Interventionsansatz wie das ES besitzt auch die Narrative Exposure Therapy (NET), die bisher zumeist zur Behandlung traumatisierter Kriegsflüchtlinge erfolgreich zum Einsatz gekommen ist (Neuner, Schauer, Klaschnik, Karunakara \& Elbert, 2004). Im Unterschied zum ES werden gemeinsam mit einem Therapeuten vergangene traumatische Erlebnisse so lange mündlich besprochen, bis die Erlebnisse in Form einer kohärenten Geschichte berichtet werden können. Der Patient wird dabei wiederholt nach seinen Gefühlen, Gedanken und körperlichen Empfindungen gefragt, bis sich bei ihm eine Habituation einstellt. Die zuletzt genannten Aspekte der kohärenten Geschichte und des Ausdrucks aktueller Gefühle und Gedanken erinnern stark an die Vorgehensweise des ES. In der letzten Sitzung der NET erhalten die Patienten ihre Biografie in schriftlicher Form.

Eine für die im Rahmen dieser Studie relevante Arbeit stellt die von Richards, Beal, Seagal und Pennebaker (2000) dar, die ES mit Insassen eines nordamerikanischen Hochsicherheitsgefängnisses durchführten. Männliche Häftlinge der psychiatrischen Abteilung mit einem Durchschnittsalter von 34,5 Jahren wurden aufgefordert, an drei Tagen jeweils 20 Minuten lang über ein belastendes Lebensereignis zu schreiben. Sechs Wochen nach dem Schreiben gaben sie im Vergleich zur Kontrollgruppe, die über ein neutrales Thema schrieb, ein höheres $\mathrm{Maß}$ selbst berichteter körperlicher Symptome an. Langfristig zeigte ein nach Tätergruppen getrennter Vergleich, dass die Gruppe der Sexualstraftäter im Vergleich zum Zeitraum vor dem ES einen verbesserten Gesundheitsstatus aufwies, der sich in einer verringerten Anzahl von Arztbesuchen auf der Station ausdrückte. Die viel diskutierten Probleme im Zusammenhang mit der Häufigkeit von Arztbesuchen als Maß für Gesundheit eines Teilnehmers werden im Kontext geschlossener Institutionen besonders deutlich. Richards und Kollegen weisen darauf hin, dass viele Inhaftierte die Krankenstation auch aus dem Bedürfnis nach Zuwendung und Abwechselung aufsuchten und eben nicht nur, weil sie körperliche Beschwerden 
haben. Die Autoren schlussfolgern, dass mit der Gruppe der Sexualstraftäter scheinbar solche Inhaftierte vom Schreiben profitierten, die in besonderem Maße stigmatisiert und seltener in der Lage seien, sich anderen anzuvertrauen. Inwieweit andere Besonderheiten dieser Gruppe wie z.B. Straflänge, Verweildauer auf der psychiatrischen Station oder Medikation eine Rolle spielen, bleibt im Rahmen dieser Studie allerdings unklar.

In einer weiteren Studie von Horn (2004) wurde das ES als eine Maßnahme eines schulbasierten Präventionsprogramms bei deutschen Schülern im Alter von 14 Jahren eingesetzt. Angenommen wurde, dass durch das Schreiben ungünstige vermeidende kognitive Verarbeitungsstile wie chronische Gedankenunterdrückung unterbrochen werden können und ES somit langfristig depressionspräventive Wirkung hat. Die Ergebnisse zeigen, dass sich durch das ES eine Verbesserung in den Bereichen Negativer Affekt, Fehltage sowie Schulnoten der Schüler ergab. Jugendliche mit einer habituellen Tendenz zur Gedankenunterdrückung profitierten jedoch kaum mehr vom Schreiben als solche, die diese Tendenz nicht zeigen.

Auch mit vielfältigen anderen nicht-klinische Probanden wie z.B. pflegenden Angehörigen (Campbell, 2003; Schwartz \& Drotar, 2004), Universitätsangestellten (Francis \& Pennebaker, 1992) oder Homosexuellen (Lewis, Derlega, Clarke \& Kuang, 2005; Swanbon, 1999) wurde das ES durchgeführt. Es zeigt sich, dass sich die Implementierung des Schreibparadigmas in unterschiedliche Kontexte, mit zahlreichen Populationen und verschiedenen inhaltlichen Schwerpunkten großer Beliebtheit erfreut.

\subsubsection{Erklärungsmodelle zur Wirkung Expressiven Schreibens}

\subsubsection{Inhibition und Exposition}

Das bereits erwähnte Inhibitionsmodell geht davon aus, dass die chronische Unterdrückung von emotionalem Ausdruck bezüglich belastender oder traumatischer Erfahrungen langfristig gesundheitsgefährdend ist, da sie wie ein überdauernder niedrigschwelliger Stressor auf den Organismus wirke (Pennebaker, 1988, 1989). Pennebaker postuliert, dass kurzfristig die Inhibition in einer Aktivierung des autonomen Nervensystems resultiere, langfristig wirke sie sich als kumulativer Stressor aus und erhöhe die Vulnerabilität für psychische Probleme und stressbedingte Krankheiten (Pennebaker, 1998). Des Weiteren würden Informationsverarbeitungsprozesse beeinträchtigt, was eine vollständige Assimilation des Erlebten behindere, so 
dass es in der Folge zu Symptomen wie Intrusionen, Grübeleien und anderen kognitiven Beeinträchtigungen kommen könne (Harber \& Pennebaker, 1992).

Ähnlich dem Konzept der Katharsis führt nach Annahme des Modells der Ausdruck unterdrückter Gedanken und Gefühle zur Stressreduktion und verbessert die physiologische sowie die psychische Gesundheit. In diesem Zusammenhang konnte gezeigt werden, dass das experimentell induzierte disclosure im Labor $\mathrm{zu}$ einer messbaren Reduktion periphärphysiologischer Parameter, wie der Erhöhung von Blutdruck, Muskelspannung und Hautleitfähigkeit führt (Pennebaker, 1989; Petrie et al., 1998). Als alleiniges Erklärungsmodell hat sich die Inhibitionstheorie jedoch als unzureichend herausgestellt, da das Aufheben inhibitorischer Prozesse nicht der einzige Wirkmechanismus des ES zu sein scheint. Probanden, die bereits über ihr Trauma gesprochen haben, profitieren offensichtlich in gleicher Weise vom Schreiben wie solche, die bisher noch nicht mit anderen über ihr traumatischen Erlebnissen sprachen (Greenberg \& Stone, 1992). Zudem konnte gezeigt werden, dass sogar das Schreiben über fiktive traumatische Erlebnisse positive gesundheitliche Effekte aufweist (Greenberg, Wortman \& Stone, 1996).

Als weitere zugrunde liegende Mechanismen sind Effekte von Reizexposition und Habituation diskutiert worden (Horn \& Mehl, 2004; Frattarolli, 2006). ES beinhaltet offensichtlich ein konfrontatives Moment, das im Schreiben und Nachdenken über das belastende Lebensereignis besteht und in der Wiederholung zur Habituation führen kann (Kloss \& Lisman, 2002; Sloan \& Marx, 2004b). So konnten Sloan \& Marx (2004a) zeigen, dass traumatisierte Studentinnen eine stärkere spätere Symptomreduktion aufwiesen, wenn sie während der ersten Schreibsitzung höhere physiologische Aktivierung zeigten, was wiederum als ein Anzeichen für stattgefundene Konfrontation gewertet wurde. Die Ergebnisse zur Wirkung des ES auf posttraumatische Belastungssymptome sind allerdings gemischt. Manche Studien zeigen eine Abnahme intrusiver und vermeidender Gedanken nach der Teilnahme an einer Schreibstudie (z.B. Klein \& Boals, 2001), andere hingegen nicht (z.B. de Moor et al., 2002; Lepore, 1997). Eine ausnahmslos expositions-basierte Wirkung des Schreibens ist aufgrund der heterogenen empirischen Befundlage daher nicht anzunehmen (vgl. Horn, 2004).

\subsubsection{Kognitive und linguistische Prozesse}

In verschiedenen Untersuchungen gaben Probanden an, durch das Schreiben profitiert zu haben, da sie eine bessere Einsicht in die Ereignisse bekommen hätten und sie besser verstehen würden als zuvor (Pennebaker, 1993). Qualitative post-hoc Analysen der Essays von Proban- 
den, die vom Schreiben profitierten, erwiesen sich als tiefer gehend, reflektierter und emotional offener als solche, die von Teilnehmern stammten, die keinerlei gesundheitliche Veränderungen zeigten (Pennebaker \& Francis, 1996). Die Entwicklung eines computerbasierten Textanalyseprogramms, des Linguistic Inquiry and Word Count (LIWC; Pennebaker, Francis \& Booth, 2001), ermöglichte eine Quantifizierung emotionaler und kognitiver Prozesse während des Schreibens. Das Programm besteht aus einem Zählalgorithmus und einer Reihe von Wörterbüchern, die ihrerseits Listen von Wörtern enthalten, die entweder grammatikalisch zusammengehören (linguistische Kategorien: z.B. Artikel, Personalpronomen, Präpositionen) oder ein bestimmtes Konstrukt repräsentieren (psychologische Kategorien: z.B. positive/negative Emotionsworte, Worte, die kognitive oder soziale Prozesse signalisieren; vgl. Horn, 2004). In einer Reanalyse der Essays von sechs Studien mittels LIWC erwiesen sich drei linguistische Prädiktoren als reliabel: eine hohe Anzahl positiver Emotionswörter, eine moderate Anzahl negativer Emotionswörter sowie ein zunehmender Gebrauch von Wörtern, die Einsicht und Kausalitätszuschreibungen reflektieren wie z.B. betrachten, wissen, verstehen, weil, deshalb (Pennebaker, Mayne \& Francis, 1997) gehen mit höheren Schreibeffekten einher. Der letztgenannte Prädiktor erwies sich statistisch als am stabilsten (Horn, 2004). Bezüglich der Anzahl der negativen Emotionswörter scheint ein Mindestmaß im Sinne der Akzeptanz negativer Aspekte des Erlebnisses im schriftlichen Ausdruck als günstig (invers uförmiger Zusammenhang). Insgesamt unterstützen diese Befunde die Annahme, dass das Erarbeiten eines kohärenten und sinnhaften Narrativs effektiv zu sein scheint. Smyth, True und Souto (2001) konnten in ihrer Studie zeigen, dass ein unorganisiertes Auflisten von Gedanken und Gefühlen bezüglich eines belastenden Ereignisses keine positiven Effekte erbrachte, dass aber im Vergleich hierzu das Verfassen eines kohärenten Narrativs zu den erwarteten gesundheitlichen Gewinnen führte.

Es erweist sich sowohl der Prozess des Formens einer kohärenten Geschichte selbst als gesundheitsförderlich (Pennebaker et al., 1997) als auch das Ziel, eine multiple Beziehung zwischen traumatischen bzw. belastenden Erlebnissen und anderen relevanten Aspekten im Lebens des Probanden herzustellen (Park \& Blumberg, 2002; Pennebaker \& Seagal, 1999; U11rich \& Lutgendorf, 2002).

\subsubsection{Emotionsregulation}

Der Befund, dass auch das ES über imaginäre traumatische Erlebnisse gesundheitlichen Verbesserungen bewirkt (Greenberg et al., 1996), lässt sich durch kognitive Prozesse nicht voll- 
ständig erklären. King \& Miner (2000) ließen ihre Probanden über mögliche Vorteile belastender Lebensereignisse schreiben. In einer weiteren Studie (King, 2001) konnte gezeigt werden, dass auch die schriftliche Auseinandersetzung mit positiven Lebenszielen z.B. die Anzahl an Arztbesuchen in der Folgezeit reduzierte und den Optimismus der Probanden steigerte.

Cameron und Nicholls (1998) demonstrierten ferner, dass eine die Selbstregulation fördernde Schreibinstruktion gleichermaßen zu gesundheitlichen Gewinnen führen kann. Wie bereits in Abschnitt 2.2.1 dargestellt, sollten studentische Probanden Probleme im College beschreiben und gleichzeitig mögliche Problemlösungen im Sinne einer Coping-Strategie vorschlagen. Die Effekte dieser von der klassischen disclosure-Instruktion abweichenden Schreibaufgaben wurden im Kontext von Selbst- und Emotionsregulationstheorien erklärt (Frattorolli, 2006). Experimentelles disclosure soll demnach die Möglichkeit bieten, sich selbst zu beobachten, und den Ausdruck sowie die Kontrolle eigener Emotionen steigern (Lepore, Greenberg, Bruno \& Smyth, 2002). Dieser Prozess führe zu einem Gefühl stärkerer Selbsteffizienz bezüglich der Regulierbarkeit eigener Gefühle. Traumata, Stressoren und andere bedeutsame Herausforderungen erscheinen langfristig kontrollierbar und zu bewältigen, was zur Verminderung negativen Affekts und gesteigertem Wohlbefinden führe. King (2002) geht davon aus, dass die Erfahrung eines traumatischen Erlebnisses den normalen Selbstregulationsprozess störe. Gefühlen käme die Aufgabe zu, im Sinne einer Feedbackschleife Rückmeldung über die persönliche Zielerreichung zu geben (affective feedback). Traumatische Erlebnisse könnten eine verlässliche Rückmeldung stören. ES biete sodann die Möglichkeit, dem Erlebten Sinn zu geben, die Ursachen für Gefühle aufzuspüren, Ziele zu klären und ein funktionierendes affektives Feedback wieder herzustellen.

Ähnlich wie King beschreiben Lepore, Greenberg, Bruno und Smyth (2002) eine mögliche Dysregulation der Gefühle im Sinne einer Über- oder Unterkontrolliertheit, ausgelöst durch traumatische Erlebnisse, als gesundheitsgefährdend. Die Wirkweisen vom ES, welches die Möglichkeit biete, extreme emotionale Reaktionen zu regulieren, seien vielfältig. In ihrem Emotionsregulationsmodell stellen die Autoren verschiedene regulative Prozesse wie Aufmerksamkeit, Habituation und Neubewertung dar, die sich positiv auf den verschiedenen Ebenen (subjektive, physiologische und Verhaltensebene) des Emotionalen Systems auswirken können. Eine detaillierte Darstellung des Emotionsregulationsmodells bieten Lepore und Smyth (2002). 


\subsubsection{Soziale Prozesse}

Das Erklärungsmodell der sozialen Integration postuliert, dass das Formen eines Narrativs traumatische Erfahrungen besser kommunizierbar mache (Pennebaker \& Graybeal, 2001). Kovac und Range (2000) konnten beispielsweise zeigen, dass Probanden, die expressiv geschrieben hatten, in der Folgezeit häufiger mit anderen darüber sprachen und im Gegenzug von Familie und Freunden mehr soziale Unterstützung erfuhren. Das Modell geht davon aus, dass experimentelles disclosure die zwischenmenschliche Interaktion verbessere und sich dadurch positiv auf die Gesundheit und das Wohlbefinden des Betroffenen auswirke (Frattarolli, 2006). Um soziales Verhalten im Alltag nach der Intervention beobachten zu können, wurde bereits am Probanden mittels digitalem Mikroaudiorekorder zeitlich getaktet Umgebungsgeräusche aufgezeichnet (Mehl, Pennebaker, Crow, Dabbs \& Price, 2001). Durch Verhaltenskodierung und linguistische Analysen ließen sich eine Vielzahl objektiver Informationen aus dem sozialen Umfeld der Probanden, ihrem Sozial- und Kommunikationsverhalten extrahieren. In einer Studie von Mehl \& Pennebaker (2003) trugen studentische Probanden den Rekorder für jeweils 48 Stunden zwei Wochen vor und zwei Wochen nach den Schreibsitzungen am Körper. Im Vergleich zur Kontrollgruppe zeigte sich, dass die Teilnehmer der Experimentalgruppe nach der Intervention im Rahmen von Konversationen signifikant mehr soziale Wörter und positive Emotionswörter sowie weniger Ärgerwörter benutzten als die Kontrollgruppe und im Vergleich zum Baseline-Zeitpunkt. Zudem konnte gezeigt werden, dass sich die Effekte des Schreibens auf das Sozialleben für männliche Probanden als stärker erwiesen als für weibliche (Mehl \& Pennebaker, 2000).

Das Modell der sozialen Integration wurde bisher nur in wenigen Studien überprüft, manche konnten dabei keine sozialen Mediatoren für gesundheitliche Effekte des ES nachweisen (z.B. Stone, Smyth, Kaell \& Hurewitz, 2000) oder neben vorhandenen Effekten hinsichtlich sozialer Beziehungen keine gesundheitlichen Verbesserungen feststellen (vgl. Frattarolli, 2006).

\subsubsection{Die Wirksamkeit des Expressiven Schreibens - metaanalytische Befunde}

\subsubsection{Ergebnisse zu kurzfristigen Parametern}

In vielen Studien zum ES wurden kurzfristige Stimmungseffekte sowie physiologische Veränderungen, die durch das Schreiben hervorgerufen wurden, untersucht. Probanden der Experimentalbedingung zeigten im Vergleich zur Kontrollgruppe direkt nach dem Schreiben eine Stimmungsverschlechterung (z.B. Francis \& Pennebaker, 1992; Gidron et al., 1996; Murray, 
Lamnin \& Carver, 1989; Pennebaker \& Beall, 1986; Sloan \& Marx, 2004a). Meistens ist diese Stimmungsverschlechterung nur von kurzer Dauer und klingt nach ca. 15 Minuten wieder ab (Murray et al., 1989). Die Verschlechterung des Affekts wurde auch als Manipulationscheck interpretiert und als ein Hinweis dafür genommen, dass die Instruktion, sich mit emotional belastenden Erlebnissen zu beschäftigen, befolgt wurde. Zumeist nimmt die Verschlechterung der Stimmung über die Sitzungen ab. Dieser Effekt wird der Wirkung von Habituation zugeschrieben (vgl. Horn, 2004).

Mehrere Studien beschreiben hinsichtlich kurzfristiger körperlicher Auswirkungen des Schreibens eine Verminderung des Blutdrucks, der Muskelspannung und der Hautleitfähigkeit bei den Probanden der Experimentalgruppe (Greenberg, Wortmann \& Stone, 1996; Pennebaker, Kiecolt-Glaser \& Glaser, 1988; Petrie, Booth, Pennebaker, Davison \& Thomas, 1995; Richards et al., 2000). In anderen Studien konnten derartige Effekte hingegen nicht gefunden werden (z.B. Greenberg \& Stone, 1992; Pennebaker \& Beall, 1986).

\subsubsection{Ergebnisse zu langfristigen Parametern}

Angesichts der großen Anzahl an Studien, die sich mit der Wirksamkeit des ES in verschiedenen experimentellen Designs und mit unterschiedlichsten Stichproben befasst haben, werden im folgenden ausschließlich Ergebnisse zu langfristigen Ergebnismaßen dargestellt, wie sie metaanalytischen Befunden zu entnehmen sind. Diese Vorgehensweise hat zum einen den Vorteil, dass die Daten in Metaanalysen bereits in aggregierter und aufgearbeiteter Form vorliegen und zum anderen nur solche Studien berücksichtigt werden, denen ein randomisiertes Kontrollgruppendesign zugrunde liegt. Im Rahmen der vorliegenden Metaanalysen zum ES wurden die vielfältigen Ergebnismaße der Primärstudien oft in Kategorien zusammengefasst (Frattarolli, 2006; Frisina, Borod \& Lepore, 2004; Harris, 2006; Meads \& Nouwen, 2005; Mogk, Otte, Reinhold-Hurley \& Kröner-Herwig, 2006; Smyth, 1998).

Als ein zentraler Indikator für langfristige gesundheitliche Auswirkungen des Schreibens stellt in vielen - vor allen frühen - Studien die Anzahl der Arztbesuche der Probanden in der Zeit vor und nach der Teilnahme an einer Schreibstudie dar. In mehreren Studien ergab sich für die Experimentalgruppe eine signifikante Abnahme in der (objektiv gemessenen) Anzahl der Arztbesuche im Vergleich zur Kontrollgruppe (z.B. Cameron \& Nicholls, 1998; Greenberg et al., 1996, Pennebaker, Barger \& Tiebout, 1989). Wird die Anzahl der Arztbesuche vom Probanden angegeben, so wird dieses Ergebnismaß hauptsächlich der Kategorie (subjektiven) Gesundheitsangaben zusammen mit Maßen wie Medikamentengebrauch und Angaben zu körperlichen Beschwerden, die über eine Reihe unterschiedlicher Fragebögen erhoben 
werden, zugeordnet. Wird die Anzahl der Arztbesuche objektiv erhoben, z.B. über universitäre Gesundheitszentren eingeholt, dann wird sie gemeinsam mit physiologische Maßen wie etwa immunologische Parameter, Blutzuckerwerte, Blutdruck oder Körpergewicht einer zweiten Kategorie, dem Physiologischen Funktionsniveau zugeordnet. So konnte beispielsweise wiederholt nachgewiesen werden, dass das ES eine langfristig förderliche Auswirkung auf das Immunsystem zeigt. Hierbei konnte gezeigt werden, dass ES sich beispielsweise positiv auf die Anzahl von Antikörpern nach einer Hepatitis B Erkrankung oder auf die immunologische Abwehr gegenüber dem Epstein-Barr-Virus auswirken kann.

Neben körperlichen Ergebnismaßen sind häufig eine Reihe psychologischer Parameter erhoben worden, wie das Ausmaß an Stress, Depression, Angst, Belastungsreaktionen oder Coping-Strategien. Diese Maße lassen sich in die Kategorie Psychische Gesundheit zusammenfassen. Auch Maße des Gesundheitsverhaltens sind in vielen Studien berücksichtigt, wie etwa das Essverhalten, der Substanzgebrauch oder das Ausmaß an physischer Aktivität. Eine weitere relevante Kategorie an Ergebnismaßen stellt das Generelle Funktionsniveau dar. Hier gehen verhaltensnahe Maße wie Schulnoten, Fehltage, kognitive Funktionen, Lebensziele oder die Qualität sozialer Beziehungen ein. Verschiedene Studien zeigten Befunde, die für eine Verbesserung dieser vielfältigen Lebensbereiche durch das ES sprechen (z.B. Cameron \& Nicholls, 1998; Crow, 2000; Evans, 2000; Horn, 2004; Francis \& Pennebaker, 1992; Klein \& Boals, 2001).

Tabelle 2 gibt einen Überblick über die Ergebnisse der sechs bisher veröffentlichten Metaanalysen zum ES. Neben den Effekten in einzelnen Ergebniskategorien sind vor allem die Gesamteffekte zu betrachten. Hier zeigt sich, dass die frühen Metaanalysen höhere Effekte nachweisen und diese als einen Hinweis dafür interpretieren, dass die Methode des ES erfolgreich ist (Frisina et al., 2004; Smyth, 1998). Diese Analysen enthalten jedoch eine geringere Anzahl an Studien als die späteren, deren Gesamteffekte deutlich geringer ausfallen (Frattarolli, 2006; Mogk et al., 2006). Mogk und Kollegen errechnen für die verschiedenen Ergebniskategorien sowie als Gesamteffekt Ergebnisse nahe Null und schlussfolgern, dass das ES keine oder nur eine vernachlässigbar geringe Wirkung aufweise. Die Metaanalysen von Meads \& Nouwen (2005) und von Harris (2006) ermitteln keinen Gesamteffekt, sondern gehen der Frage nach, inwieweit die Schreibintervention hinsichtlich bestimmter Ergebniskategorien (z.B. ausgewählte psychische Gesundheitsparameter) wirksam ist, bzw. inwieweit bestimmte Probandengruppen (Gesunde vs. Beeinträchtigte) vom Schreiben profitieren. So gelangt Harris zu der Auffassung, dass das Schreiben über belastende Erlebnisse sich für gesunde Probanden durchaus als lohnend erweisen kann. Für körperlich oder psychisch beeinträch- 
tigte Probanden zeigten sich jedoch keine Effekte. Diese bezieht der Autor ausschließlich auf die Häufigkeit von Arztbesuchen (engl. „Health Care Utilization“, HCU), wobei er betont, dass die Abnahme von Arztbesuchen keinesfalls gleichzusetzen sei mit einem objektiv verbesserten Gesundheitsstatus. Meads \& Nouwen (2005) kommen im Rahmen ihrer Analyse hinsichtlich des objektiven Maßes HCU zu dem Ergebnis, ,that there is no difference in objectively measured HCU overall for the intervention group compared with control at followup“. Wobei zwischen den untersuchten Gruppen beträchtliche Heterogenität bestünde, so dass „the meta-analysis may have shown a clearer trend for the intervention group for healthy participants..." (S. 156). Eine Annahme, die von Harris bestätigt wurde.

Da in die metaanalytischen Befunde der Studie von Frattorolli (2006) bisher die größte Anzahl von Einzelstudien $(\mathrm{n}=146)$ eingegangen sind und ein relativ hoher Anteil von unpublizierten Studien $(n=31)$ einbezogen wurde, wird im folgenden vorwiegend Bezug auf die Ergebnisse dieser Analyse genommen. Die Ziele der Metaanalyse von Frattarolli bestanden zum einen im Ermitteln einer generellen Effektgröße von ES und zum anderen in der Bestimmung potentiell moderierender Einflussgrößen der experimentellen Variationen (vgl. hierzu auch 2.2.3.3). 
Tabelle 2

Metaanalytische Befunde für Expressives Schreiben: Stichprobengröße, Gesamteffekt, Effekte der Ergebniskategorien und der Moderatoren

\begin{tabular}{|c|c|c|c|c|c|c|c|c|c|c|c|c|}
\hline \multirow{2}{*}{ Studie } & \multirow[b]{2}{*}{$k$} & \multirow[b]{2}{*}{$n$} & \multirow[b]{2}{*}{$\begin{array}{c}\text { Gesamt- } \\
\text { effekt }\end{array}$} & \multirow[b]{2}{*}{$p$} & \multirow[b]{2}{*}{ Modell } & \multicolumn{5}{|c|}{ Effekte nach Ergebniskategorien } & \multirow{2}{*}{\multicolumn{2}{|c|}{$\begin{array}{l}\text { Effekte von Moderatoren } \\
\text { untersucht } \quad \text { Ergebnis }\end{array}$}} \\
\hline & & & & & & $\mathbf{A}$ & $\mathbf{B}$ & $\mathbf{C}$ & $\mathbf{D}$ & $\mathbf{E}$ & & \\
\hline Smyth, 1998 & 13 & 806 & $\begin{array}{l}.47^{\mathrm{a}} \\
.23^{\mathrm{b}}\end{array}$ & .00 & fixed & $\begin{array}{l}.66^{* * * a} \\
.31^{\mathrm{b}}\end{array}$ & $\begin{array}{l}.68^{* * * a \mathrm{a}} \\
.32^{\mathrm{b}}\end{array}$ & $\begin{array}{l}.42^{* * * a} \\
.21^{\mathrm{b}}\end{array}$ & $\begin{array}{l}.03^{\mathrm{a}} \\
.01^{\mathrm{b}}\end{array}$ & $\begin{array}{l}.33^{* * * a} \text { a } \\
.16^{\mathrm{b}}\end{array}$ & ja & $\begin{array}{l}\text { Höhere Effekte bei: } \\
\text { - studentischer SP (A) } \\
\text { - männlichen SP }(d) \\
\text { - längeren Sitzungsintervallen } \\
\text { - aktuelle Traumata (A) }\end{array}$ \\
\hline $\begin{array}{l}\text { Frisina et al., } 2004 \\
\text { Klinische Population }\end{array}$ & 9 & 496 & $\begin{array}{l}.19^{\mathrm{a}} \\
.10^{\mathrm{b}}\end{array}$ & .04 & fixed & $.074^{\mathrm{a}}$ & $.211 * \mathrm{a}$ & & & & ja & $\begin{array}{l}\text { - Keine Moderatoreffekte } \\
\text { nachweislich }\end{array}$ \\
\hline Meads et al., 2005 & 61 & & & & random & \begin{tabular}{|c|} 
Neg. Affekt: \\
$0.37^{\mathrm{c}}$ \\
$(\mathrm{CI}=0.12,0.62)$ \\
Depression: \\
$0.22^{\mathrm{c}}$ \\
$(\mathrm{CI}=+0.05,+0.40)$ \\
\end{tabular} & $\begin{array}{c}\text { nur HCU: } \\
-0.55^{\mathrm{c}} \\
(\mathrm{CI}=-1.13 \\
+0.03)\end{array}$ & $\begin{array}{c}\text { nur HCU: } \\
-0.06^{\mathrm{c}} \\
(\mathrm{CI}=-0.26 \\
+0.13)\end{array}$ & & $\begin{array}{c}0.25^{\mathrm{c}} \\
(\mathrm{CI}=0.09,0.41) \\
3.04^{*} \mathrm{~d}\end{array}$ & nein & \\
\hline $\begin{array}{l}\text { Harris, } 2006 \\
\text { getrennt nach } \\
\text { Gesunden } \\
\text { physisch- u. } \\
\text { psychisch Beein- } \\
\text { trächtigten }\end{array}$ & $\begin{array}{l}14 \\
6 \\
10\end{array}$ & $\begin{array}{r}1394 \\
304 \\
538\end{array}$ & & & random & & & $\begin{array}{c}\text { nur HCU: } \\
\\
\\
0.16^{\mathrm{e}} \\
(\mathrm{CI}=0.02,0.31) \\
0.21^{\mathrm{e}} \\
(\mathrm{CI}=-0.030 .43) \\
0.06^{\mathrm{e}} \\
(\mathrm{CI}=-0.12,0.24)\end{array}$ & & & ja & $\begin{array}{l}\text { - Gesunde: geringer } \\
\text { Gesamteffekt außerhalb } \\
\text { des Labors } \\
\text { - psychisch Beeinträchtigte: } \\
\text { geringerer Effekt bei } \\
\text { ansteigender Anzahl von } \\
\text { Schreibsitzungen }\end{array}$ \\
\hline Mogk et al., 2006 & 30 & & $\begin{array}{c}-.07^{\mathrm{e}} \\
(\mathrm{CI}= \\
-0.16,0.03) \\
\end{array}$ & & random & $\begin{array}{c}-.12 \\
(\mathrm{CI}=-0.25,0.00)\end{array}$ & $\begin{array}{c}.05 \\
(\mathrm{CI}=-0.16 \\
0.06) \\
\end{array}$ & & & & nein & \\
\hline Frattarolli, 2006 & 146 & 10703 & $.075^{\mathrm{b}}$ & .00 & random & $.056^{* * \mathrm{~b}}$ & $.059 * \mathrm{~b}$ & $.07 * * \mathrm{~b}$ & .01 & $.046^{*}$ & ja & $\begin{array}{l}\text { Höhere Effekte bei: } \\
\text { - publizierten Studien, } \\
\text { - höherem Stresslevel } \\
\text { - kurzem FU }(<1 \text { Monat }) \\
\text { - > } 3 \text { Sitzungen, > } 15 \text { Min. } \\
\text { - kurz zurückliegendem Ereignis } \\
\text { - Instruktion m. Beispielen } \\
\text { u. direkten Fragen }\end{array}$ \\
\hline
\end{tabular}

Anmerkungen. Alle Metaanalysen enthalten ausschließlich RCT's. Ergebniskategorien sind A= psychische Gesundheit, B= physiologisches Funktionsniveau, C= subjektive Gesund-

heitsangaben, $\mathrm{D}=$ Gesundheitsverhalten, $\mathrm{E}=$ generelles Funktionsniveau; $\mathrm{k}=$ Anzahl der eingegangenen Studien; HCU= Health Care Utilization; CI = Konfidenzintervall.

${ }^{a}$ Cohen's $d$. b Korrelationskoeffizient $r$. ${ }^{c} \mathrm{WMD}=$ weighted mean differences. ${ }^{\mathrm{d}} \mathrm{z}$-Wert. ${ }^{\mathrm{e}}$ Hedges's $g(95 \% \mathrm{CI}) .{ }^{\mathrm{f}}$ berichtet werden lediglich signifikante Moderatoren.

$* p \leq .05, * * \mathrm{p}<.001, * * * p<.0001$. 
Als Einschlusskriterien für die Auswahl der Primärstudien galten:

- die Studie ist eine Variation des originalen Schreibparadigmas von J.W. Pennebaker

- es handelt sich um ein randomisiertes Kontrollgruppendesign (RCT)

- es liegen Daten einer Follow-up-Erhebung vor

- die Ergebnisse der Studie sind erstmalig veröffentlicht

- die Gruppenzuordnung ist nicht mit anderen Variablen konfundiert

Für die Ergebniskategorie Psychische Gesundheit ermittelt Frattarolli eine ungewichtete Effektgröße von $r=.056(p<.001)$. Vor allem in den Variablen Stress $(r=.102)$, Depression $(r=$ .073) und Befindlichkeit $(r=.045)$ ergeben sich signifikante Verbesserungen durch das Schreiben. Im Bereich der physiologischen Parameter erweisen sich die Immunparameter ( $r=$ .128) als signifikant verändert, wobei für viele andere Variablen die zu berücksichtigende Studienanzahl zu gering ist. Die subjektiven Angaben zur Gesundheit weisen einen Gesamteffekt von $r=.072(p<.001)$ auf, wobei sich spezifische Krankheitsparameter $(r=.128)$ und das Krankheitsverhalten ( $r=$. 073) als signifikant verbessert erweisen. Die Effektstärke für den Bereich Gesundheitsverhalten fällt mit $r=.007$ nicht signifikant aus. In der Kategorie Generelles Funktionsniveau mit einer Effektstärke von $r=.046(p<.05)$ zeigen sich arbeitsbezogene Maße $(r=.084)$, soziale Kontakte $(r=.058)$ und kognitive Leistungen $(r=.058)$ als signifikant. Der größte Effekt im Rahmen der Analyse mit $r=.159(\mathrm{p}<.00001)$ zeigt die Kategorie Subjektive Beurteilung des Schreibeffekts . Hier werden Maße wie die positive Haltung gegenüber der Intervention $(r=.270)$ und der Versuch, dem Ereignis weiterhin Sinn zuzumessen $(r=.132)$ erfasst.

Der von Frattarolli ermittelte Gesamteffekt von $r=.075$ entspricht einem $d=.15$ nach Cohen (1988) und ist laut Konvention zunächst als klein zu bewerten und fällt deutlich kleiner als die von Smyth (1998) und Frisina et al. (2004) gefundenen Effekte aus. Dies ist sicherlich teilweise auf den im Vergleich zu allen anderen Metaanalysen höheren Anteil unpublizierter Studien in der Analyse von Frattarolli, die tendenziell geringere Effekte aufweisen, zurückzuführen. Neben der konventionellen Beurteilung eines Effektes sollte laut Frattarolli aber auch die praktische Bedeutung eines Effektes, sein Kosten-Nutzen-Verhältnis berücksichtigt werden. Unter der Voraussetzung, dass die Schreibintervention praktisch kostenfrei, nicht-invasiv sowie flexibel sei und zudem von den Probanden als hilfreich beurteilt werde, sei jeder Effekt über Null bemerkenswert, so die Autorin. Außerdem führt sie einen Vergleich mit verschiedenen anderen Forschungsbereichen an, um deutlich zu machen, dass auch geringe Effekte von Bedeutung seien. So weise beispielsweise die tägliche Einnahme von Aspirin zur Präven- 
tion von Herzinfarkten einen Effekt von $\mathrm{r}=.034$ auf und in der erziehungswissenschaftlichen Forschung würden Effekte von .05 als nennenswerte Lern- und Leistungsverbesserungen gewürdigt (vgl. Frattarolli, 2006, S. 851). Selbstverständlich könnten von einer kurzen Intervention wie dem Expressiven Schreiben keine so hohen Effekte zu erwarten sein wie etwa von Psychotherapie: „To have arrived at any other result should cause the reader to be suspicious“ (ebd., S. 853). Bei der resultierenden Effektgröße handele es sich überdies um eine durchschnittliche Größe, in die die Ergebnisse von Studien eingegangen seien, die nicht optimal durchgeführt worden seien. Unter optimalen Bedingungen (z.B. bei hoher Dosierung, privatem Setting, spezifischer Instruktion) errechnet Frattarolli einen Effekt von .20 für das ES. Da sich die Studien so unterschiedlich gestalten, sei es wichtig, so die Autorin, den Fokus zusätzlich auf mögliche Moderatorvariablen zu richten, um zu ermitteln, unter welchen Voraussetzungen das Schreiben effektiv ist.

\subsubsection{Ergebnisse zu Moderatoren}

Im Rahmen der Analyse zugrunde liegender Wirkmechanismen des ES sind wiederholt Versuche gemacht worden, Faktoren, die differentiellen Einfluss auf die Effektivität der Intervention zeigen können, zu identifizieren. So stellen Sloan und Marx (2004b) in ihrem Review fest: ,... more attention needs to be directed toward understanding why it works and for whom this approach might be best suited“" (S. 121). Je nach theoretischem Konzept wurden verschiedene interindividuelle Unterschiede für differentielle Effekte des Schreibens verantwortlich gemacht. So wurde der Befund, dass optimistisch eingestellte Probanden stärker als pessimistisch eingestellte vom Schreiben profitieren, im Rahmen des Selbstregulationsansatzes auf den unterschiedlich habituell verankerten Umgang mit affektiven Situationen zurückgeführt (Cameron \& Nicholls, 1998; Horn, 2004). Im Sinne des Inhibitionsansatzes wurde das Ausmaß zynischer Feindseligkeit als affektive Einstellungsvariable untersucht, wonach angenommen wurde, dass feindselige Probanden weniger Selbstöffnung zeigen und daher mehr vom Schreiben (ohne soziales Feedback) profitieren können (Christensen \& Smith, 1993; Christensen et al., 1996). Auch die Persönlichkeitsvariable Alexithymie ist wiederholt hinsichtlich ihrer möglichen Moderatoreigenschaft untersucht worden. Die Studien von Paez und Kollegen (1999) sowie von Solano und Kollegen (2003) konnten zeigen, dass Probanden mit hoher Ausprägung an Alexithymie (gemessen mittels Toronto Alexithymieskala TAS) mehr vom ES profitieren als solche mit niedrigen Werten (Paez, Velasco \& Gonzales, 1999; Solano, Donati, Pecci, Persichetti \& Colaci, 2003). Wird dem ES eine emotionsregulatorische Wirkweise unterstellt, so profitieren hoch-alexithyme Probanden deshalb, weil sie lernen, ihre 
Schwierigkeiten bei der Wahrnehmung und Benennung eigener Gefühle zu überwinden, diese auszudrücken und zu reflektieren. Andere Studien finden solche Zusammenhänge nicht (Kröner-Herwig et al., 2004). Im Rahmen der umfangreichen metaanalytischen Betrachtung etwaiger Moderatorvariablen Expressiven Schreibens findet Frattarolli (2006) ebenfalls keinen nennenswerten Effekt für die Variable Alexithymie (vgl. Tabelle 3). Gleiches gilt für die Variablen Alter und Geschlecht, letzteres wurde zuvor in der Metaanalyse von Smyth (1998) noch als ein moderierender Faktor ermittelt, wonach männliche Probanden stärker vom Schreiben profitierten als weibliche.

Neben personen- und dispositionsbezogenen Variablen sind zahlreiche weitere Moderatoren untersucht worden. Die metaanalytischen Untersuchungen zeigen im Hinblick auf einige Moderatorvariablen kein einheitliches Ergebnis (z.B. Anzahl der Sitzungen, psychische Beeinträchtigung der Probanden oder Publikationsstatus der Studie). Im Gegensatz zur Metaanalyse von Smyth (1998) kommt Frisina (2006) in der bisher umfangreichsten Analyse zu dem Ergebnis, dass nicht-studentische Populationen hinsichtlich psychischer Gesundheitsparameter am meisten vom Schreiben profitieren, wobei hier das Setting des Schreibens eine Rolle spielt, da sich Schreibsitzungen, die zuhause durchgeführt worden sind, als wirkungsvoller erwiesen haben. Studenten haben in nahezu keiner Studie bei sich zuhause geschrieben und gehören deshalb nicht zu der Probandengruppe, die gut vom ES profitiert. Neben dem zu vernachlässigenden Geschlechtereffekt haben nach Frattarollis Erkenntnissen auch die Sitzungsintervalle sowie der Modus der Selbstöffnung (sprechen vs. schreiben) keinen Einfluss auf die Effektivität der Intervention. 
Tabelle 3

Metaanalytische Befunde zu Moderatorvariablen vom Expressiven Schreiben

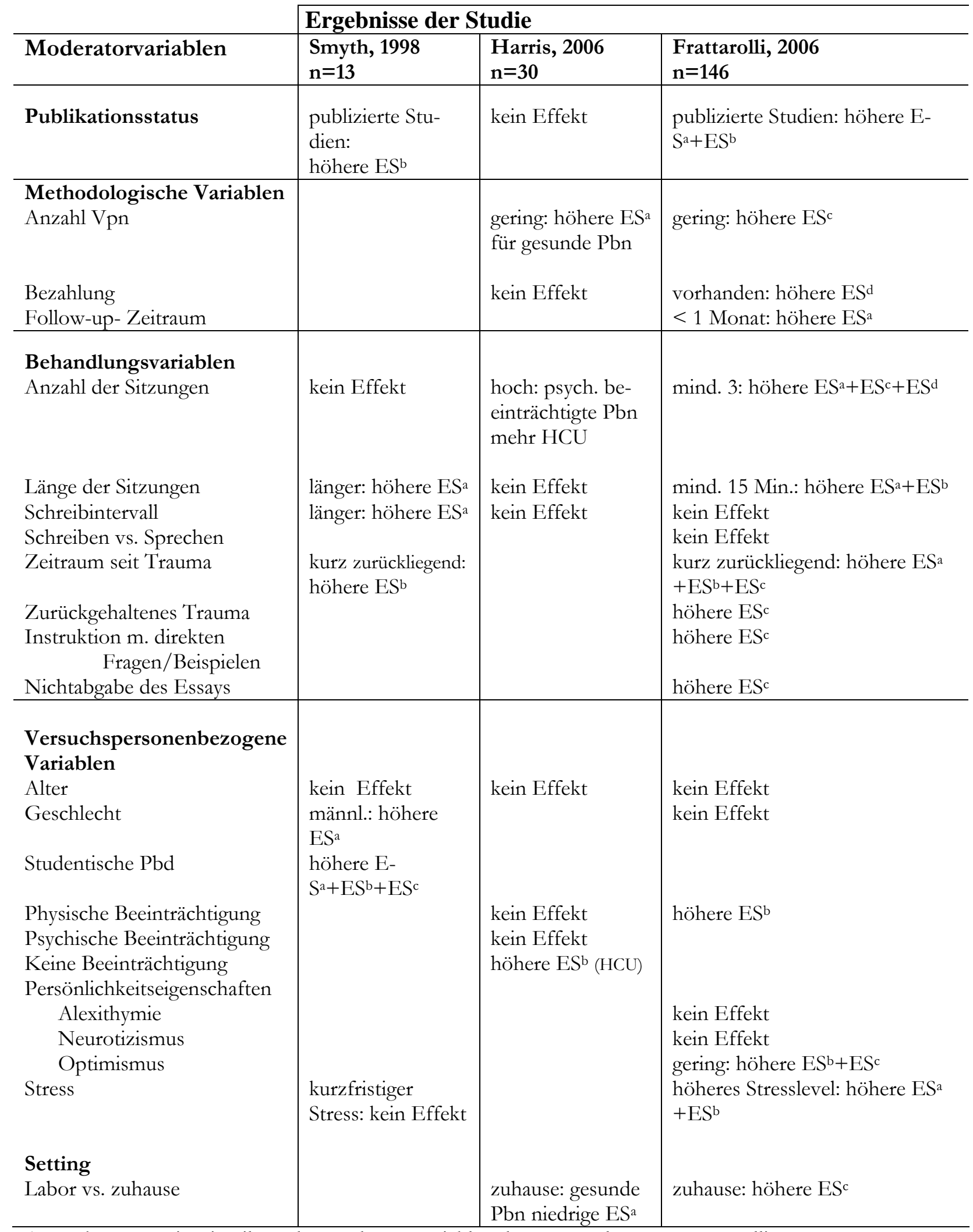

Anmerkungen. Die Einteilung der Moderatorvariablen der Metaanalyse von Frattorolli entnommen „Experimental disclosure and its moderators: A meta-analysis, “, 2006, Psychological Bulletin, 132, p.854. ESa $=$ Effektstärke, gesamt; ES $=$ Effektstärke, physische Parameter (objektiv u. subjektiv); ESc $=$ Effektstärke, psychische Parameter; ESd = Effektstärke, subjektive Einschätzung des Schreibens; HCU= Health Care Utilization; 
Um aus diesen Befunden Schlussfolgerungen für eine zukünftige angemessene Anwendung und Umsetzung des Schreibparadigmas zu ziehen, lassen sich ihre Ergebnisse folgendermaßen zusammenfassen:

Die Effektivität der Intervention ist höher, wenn die Probanden physische Beschwerden haben, eine (trauma)- belastete Vorgeschichte aufweisen, sie zuhause in privater Atmosphäre nach Instruktion mit direkten Aufforderungen (Fragen und Beispielen) mindestens drei Mal und jeweils mindestens 15 Minuten lang schreiben. Beim zu wählenden Inhalt sind zeitnahe Erlebnisse vorzuziehen, die der Proband zuvor am besten noch nicht mit anderen besprochen hat. Die Anzahl der Probanden pro Studie sollte nicht zu groß sein, die Teilnehmer sollten für ihre Teilnahme überdies einen Schadensaufwand erhalten. Der Follow-up-Zeitraum sollte einen Monat nicht überschreiten und die geschriebenen Essays besser nicht vom Versuchsleiter eingesammelt werden (vgl. Frattarolli, 2006).

\subsubsection{Expressives Schreiben als intramurale Interventions- maßnahme}

Im folgenden werden relevante Aspekte erörtert, die für die Implementierung einer Schreibintervention im Jugendstrafvollzug sprechen. Im einzelnen handelt es sich dabei um Aspekte der Klientel, des Settings und der Möglichkeiten, die ES in der Arbeit mit jugendlichen Straftätern bietet.

Unabhängig von der Art der begangenen Straftat kann angenommen werden, dass eine Haftstrafe für jeden Inhaftierten eine starke Veränderung der Lebenssituation darstellt. Gerade erstinhaftierte jugendliche und heranwachsende Gefangene müssen sich zunächst an die stark reglementierten Strukturen des Strafvollzuges gewöhnen und eine gewisse Anpassungsleistung erbringen. In dieser neuen Situation kann neben den Einschränkungen durch die Haft auch die Möglichkeit gesehen werden, sich zu verändern und neue Lebensperspektiven zu entwickeln. Es konnte gezeigt werden, dass die überwiegende Mehrheit jugendlicher Inhaftierter durchaus normkonforme Lebensziele zu besitzen scheint, es wohl aber bei der Realisierung dieser Ziele an persönlichen und sozialen Kompetenzen mangelt (Suhling, 2005). Wie bereits unter Abschnitt 2.1.1 dargestellt, handelt es sich bei jugendlichen und heranwachsenden Inhaftierten um eine hochselektive Gruppe, die extrem sozial abweichendes Verhalten in chronifizierter Form gezeigt hat, gehäuft Auffälligkeiten im Persönlichkeitsbereich sowie überdurchschnittlich oft Alkohol- und Drogenprobleme aufweist und von einer Vielzahl psychosozialer Probleme gekennzeichnet ist (z.B. Schulversagen, psychische und physische Be- 
einträchtigungen). Junge Inhaftierte gehören $\mathrm{zu}$ einer besonders vulnerablen Gruppe Jugendlicher mit nachweislich überdurchschnittlicher Belastungssymptomatik und einer damit verbundenen (trauma-)belasteten Vergangenheit. Wie erwähnt, gehen jedoch Leidensdruck und Behandlungsbereitschaft im klassischen Verständnis von Therapiemotivation gerade bei deutlicher Sozialisationsbelastung nicht automatisch miteinander einher. Bei jeder Art von intramuraler Intervention bestehen überdies Probleme der Fremdmotiviertheit und hoher Abbruchraten.

Das Expressive Schreiben bietet in Form des klassischen Schreibparadigmas die Möglichkeit, sich trotz möglicher Scham- oder Angstgefühle mit belastenden Erfahrungen auseinander zu setzen. Darüber hinaus bietet es im Gegensatz zur verbalen Selbstöffnung einer anderen Person gegenüber den Vorteil, vollkommen anonym durchführbar und frei von interpersoneller Rückmeldung zu sein. Es konnte gezeigt werden, dass ES problemlos in verschiedenen Settings (z.B. Klinik, zuhause, Schule) und in unterschiedlichen Lebenskontexten (bei Krankheit, während akuter Trauer, zu Beginn des Studiums) eingesetzt werden konnte. Die Schwelle an einer solchen Kurzintervention teilzunehmen, ist vergleichsweise gering und die Bereitschaft zur Mitarbeit dementsprechend recht groß.

Die Darstellung der verschiedenen intramuralen Interventionen und ihrer Wirksamkeit hat gezeigt, dass Psychotherapie als Behandlungsmaßnahme im Strafvollzug eine Ausnahme darstellt. Interventionsmethoden, die eigens zur Straftäterbehandlung entwickelt wurden, wie beispielsweise das Reasoning and Rehabilitation Program basieren auf dem theoretischen Konzept, antisoziale Denkmuster und Einstellungen wirkten im Sinne kognitiver Defizite und stellten somit einen wesentlichen Risikofaktor für die Aufrechterhaltung delinquenten Verhaltens dar (vgl. Abschnitt 2.1.2.3 und 2.1.3). Sicherlich kann eine wesentlich kürzere und somit weniger intensive Intervention wie das ES im Vergleich zu einem umfangreichen therapeutischen Behandlungsprogramm, das über einen langen Zeitraum im multiprofessionellen Team durchgeführt wird, kaum den Anspruch erheben, direkt oder indirekt rückfallvermindernd zu wirken. Es hat jedoch den Vorteil, in der Gestaltung variabel und wenig ressourcenintensiv zu sein. So ist vorstellbar, dass ES eingesetzt wird, um im Sinne einer ersten reflektiven Auseinandersetzung und Bewältigung mit dem Vergangenen, vorbereitende Prozesse für eine kognitive Umstrukturierung in Gang zu setzen. Neben dem Schreiben über ein belastendes Lebensereignis kann es zusätzlich im Sinne einer schriftlichen Tataufarbeitung Aspekte berücksichtigen, die mit dem Risikofaktor antisoziales Denken in Zusammenhang stehen. Die Auseinandersetzung mit der eigenen Straffälligkeit und dem/den Delikte/n wird bei der Aufarbeitung der Straftat als eine wichtige Voraussetzung für eine weiterführende therapeutische 
Arbeit an verschiedenen Risikofaktoren gesehen, die durch das Herausarbeiten des (typischen) Musters des Deliktablaufs zutage treten können und dem Inhaftierten häufig zum ersten Mal bewusst werden (Hinrichs, 1993; Hagemann, 2003). Hierbei handelt es sich zum Beispiel um kognitive Verzerrungen, die Relevanz der geführten Lebensweise, die sozialen Beziehungen oder der frühere Drogenkonsum (Freese, 2005). Der Inhaftierte soll dazu befähigt werden, zukünftig potentielle Krisensituationen zu erkennen und darüber hinaus sozial verträgliche Bewältigungsmöglichkeiten und Handlungsalternativen zu erlernen. Gefühle und Gedanken, die in Zusammenhang mit der eigenen Täterschaft stehen, können durchaus schambesetzt sein und das Selbstbild bedrohen (Simons, 1996). Daher stellt die Methode des ES gerade zu Beginn der Haftzeit und einer möglichen Auseinandersetzung mit der delinquenten Vergangenheit ein niedrig-schwelliges Angebot dar. Da es sich bei der Tataufarbeitung nicht um einen Zielzustand, sondern vielmehr um einen Prozess handelt, kann das ES als ein erster Schritt zur Auseinandersetzung verstanden werden, der dem Inhaftierten die Möglichkeit bietet, sich auf seine persönliche Weise und in seiner eigenen Geschwindigkeit mit seiner Vergangenheit zu konfrontieren. Hierbei wird er genau wie im klassischen Paradigma aufgefordert, seine tiefsten Gedanken und Gefühle zu äußern.

Die Variante, das Schreibthema vorzugeben, ist, wie bereits dargestellt, im Rahmen vieler ESStudien realisiert worden (Batten, Follette, Hall \& Palm, 2002; King, 2001; Snyder, Gordon \& Baucom, 2004). In verschiedenen Schreibstudien sind Themen wie z.B. positive Lebensziele instruiert worden, die in keinem direkten Bezug zu belastenden oder traumatischen Erfahrungen einer Person stehen. Dass dennoch positive Effekte resultierten, wurde hierbei im Kontext möglicher selbstregulatorischer Wirkungweisen des ES diskutiert (Greenberg et al., 1996; King, 2000). Auch die Strukturierung der Schreibinstruktion über die Sitzungen hinweg ist in einigen Studien umgesetzt worden (Cameron \& Nicholls, 1998, Gidron et al., 2002; Kröner-Herwig et al., 2004). Dies u.a. mit dem Ziel, eine möglichst kohärente Geschichte der Erlebnisse mit einem Anfang und einem Ende zu erzeugen. Smyth, True und Souto (2001) instruierten in ihrer Studie die Probanden entweder in fragmentierter oder aber in narrativer Form über ein traumatisches Erlebnis zu schreiben. Dabei wurde deutlich, dass nur das Formen eines kohärenten Narrativs die gewünschten gesundheitlichen Effekte des ES mit sich brachte. Auch im Rahmen dieser Studie erhielten alle Teilnehmer der Schreibgruppe Tataufarbeitung (TA) sowie die der Gruppe Belastendes Lebensereignis (BE) an jedem Schreibtermin eine strukturierte Instruktion, die jeweils einen unterschiedlichen Fokus auf die Erlebnisse richtet: Am ersten Schreibtag soll sich das Geschehene vor Augen geführt werden, um am zweiten Tag über die Zeit nach der Straftat und die Folgen dieser (für die eigene Per- 
son und andere) zu schreiben. In der dritten Schreibsitzung soll das Erlebte abschließend bewertet und überlegt werden, wie man selbst zukünftig in ähnlichen Situationen reagieren würde (vgl. Anhänge A5-A7). Der Einsatz strukturierter Schreibinstruktionen, die sehr konkrete Aufforderungen enthalten, sind im Kontext verschiedener Studien zum ES bereits erfolgreich eingesetzt worden (Cameron \& Nicholls, 1998; Gidron, Duncan, Lazar, Biderman, Tandeter \& Shvartzman, 2002; Schoutrop, Lange, Hanewald, Davidovich \& Salomon, 2002). Einige Autoren gehen davon aus, dass ein schrittweises Vorgehen mit expliziten Anweisungen den Effekt der Konfrontation wie auch der kognitiven und emotionalen Verarbeitungsprozesse erhöhe. Überdies konnten Campbell und Pennebaker (2003) mittels inhaltsanalytischer Auswertungen der Essays dreier Studien zeigen, dass ein über die Schreibsitzungen veränderter Schreibstil vorteilhaft zu sein scheint. Probanden, die im Verlauf der Sitzungen im schriftlichen Ausdruck sich selbst sowie anderen gegenüber eine veränderte Perspektive einnahmen, zeigten die größten gesundheitlichen Verbesserungen. Eine rückblickende Bewertung der Geschehnisse in der letzten Schreibsitzung bietet daher im Rahmen dieser Studie den Inhaftierten die Möglichkeit, in der Beschreibung des belastenden Lebensereignisses bzw. der Straftat ein Ende zu definieren und somit die „Geschichte“ zu einem ersten Abschluss zu bringen und gleichzeitig einen Ausblick in die Zukunft zu formulieren. Der durch die Instruktion veränderte Fokus jeder Sitzung soll überdies einen Perspektivenwechsel erleichtern.

\subsection{Ziele der Studie}

\subsubsection{Adaptation und Implementierung der Schreibintervention im Jugendvollzug}

Um unter den institutionellen Rahmenbedingungen einer Jugendstrafanstalt eine Schreibintervention durchzuführen, sind neben den strukturellen Bedingungen des Strafvollzuges auch die Besonderheiten der Klientel zu berücksichtigen. Zunächst muss gewährleistet sein, dass die Teilnahme am „Schreibprojekt“ freiwillig erfolgt und für den Inhaftierten nicht mit führungsund lockerungsbedingten Vergünstigungen einhergeht. Die Maßnahme ist deshalb deutlich gekennzeichnet als eine Veranstaltung, die im Rahmen eines Forschungsprojektes als ein zusätzliches, von außen in die Haftanstalt gebrachtes Angebot durchgeführt wird.

Ein wesentliches Ziel dieser Studie besteht darin, den Teilnehmern beider Experimentalgruppen (TA und BE) die Möglichkeit zu geben, zwischen den Essayinhalten und anderen Lebensaspekten (Zeit vor der Inhaftierung, Haftzeit und Zukunftsperspektiven) Beziehungen 
herzustellen. Es wird angenommen, dass ES bei den Inhaftierten die Fähigkeit zur Selbstbeobachtung schulen und auf diese Weise den Ausdruck und die Kontrolle eigener Emotionen steigern kann. Bestenfalls führt eine verbesserte Emotionsregulation langfristig zu einer Verminderung negativen Affekts und zu gesteigertem psychischen und physischen Wohlbefinden.

Neben der Annahme, ES verbessere die Fähigkeit zur Emotionsregulation wird im Sinne des Erklärungsmodells der sozialen Integration vermutet, dass sich ebenfalls eine Verbesserung zwischenmenschlicher Interaktionen einstellen kann (vgl. 2.2.2.4). In Anlehnung an die Studie von Horn (2004) werden auch im Rahmen dieser Studie verhaltensnahe Maße erhoben, da sie sich in Form von Fehltagen und Schulnoten der Studienteilnehmer als ein geeignetes Maß zur Erfassung möglicher (Verhaltens-)Veränderungen erwiesen haben. Im Falle der Jugendanstalt bietet sich die monatliche Führungsbenotung, die durch die Mitarbeiter der Anstalt vergeben wird, als ein Auskunftsmaß für das Sozial- und Leistungsverhalten jedes Inhaftierten an.

Bei der Überprüfung der Wirksamkeit von ES hat sich überdies gezeigt, dass es bei der Implementierung und Auswertung einer Schreibstudie wichtig ist, differenzielle Aspekte der Wirksamkeit zu berücksichtigen. Aus diesem Grund ist es angezeigt, eine post-hoc Analyse der Essays der Teilnehmer durchzuführen. Nur auf diese Weise können relevante prozessuale Faktoren des ES eruiert und mögliche Subgruppen ,erfolgreicher“ Schreiber erkannt werden. Hierbei fungieren Essaymerkmale wie beispielsweise die Anzahl positiver Emotionswörter als Moderatoren gesundheitsförderlichen Schreibens. Für eine über die mögliche Moderatorfunktion hinausgehende umfassende Darstellung der Ergebnisse der quantitativen Inhaltsanalyse sei auf die Diplomarbeit von Studtmann-Pieper verwiesen.

Bezüglich der Variablen, die laut metaanalytischen Befunden mögliche Schreibeffekte erhöhen, ergibt sich im Hinblick auf die Variablen des Settings und der Klientel dieser Studie ein gemischtes Bild (vgl. Frattarolli, 2006). Inhaftierte Jugendliche weisen nicht notwendigerweise mehr physische Beschwerden auf als die üblichen Teilnehmer an einer Schreibstudie. Überdies schreiben sie im Rahmen dieser Studie nicht zuhause (wobei die Haftanstalt für die Zeit der Strafverbüßung durchaus als eine Art zuhause betrachtet werden könnte). Sie werden außerdem nicht explizit dazu aufgefordert, über ein zeitnahes Erlebnis zu schreiben (was sich im Falle der Straftataufarbeitung auch als schwierig gestaltet hätte). Der gewählte Follow-upZeitraum übersteigt mit acht Wochen außerdem die sich als optimal erwiesene und zugleich recht kurze Einmonatsfrist. Die inhaltsanalytische Auswertung der Essays macht es außerdem 
nötig, dass die Essays nicht beim Teilnehmer verbleiben können. Sicher kann hingegen davon ausgegangen werden, dass die Jugendlichen eine nicht weniger belastete Vergangenheit aufweisen als nicht-inhaftierte Gleichaltrige (Enzmann \& Greve, 2001). Die verwendete Schreibinstruktion enthält direkte Aufforderungen, die Teilnehmer werden außerdem aufgefordert, über Aspekte der Straftat bzw. des belastenden Lebensereignisses zu schreiben, die sie zuvor noch nicht ausführlich mit jemandem besprochen haben. Auch Häufigkeit und Dauer der Sitzungen erfüllen die Kriterien einer optimal gestalteten Studie. Sicherlich besteht ein Ziel dieser Studie auch darin, herauszufinden, ob und inwieweit ES von Inhaftierten angenommen wird und mit ihnen durchgeführt werden kann. Im zweiten Schritt soll überprüft werden, ob somit die Voraussetzungen geschaffen sind, ES als eine sinnvolle und effektive Maßnahme im Jugendstrafvollzug einzusetzen.

\subsubsection{Hypothesen}

Aus dem zuvor Dargestellten werden im Rahmen dieser Studie folgenden Hypothesen aufgestellt:

1) Die beiden Experimentalgruppen (Schreiben über Straftat bzw. über belastendes Lebensereignis) unterscheiden sich hinsichtlich der kurzfristigen stimmungsbezogenen Auswirkungen des Schreibens von der Kontrollgruppe. Die Teilnehmer der Experimentalgruppen zeigen direkt nach dem Schreiben eine negativere Stimmung als die Teilnehmer der Kontrollgruppe.

2) Die Gruppen unterscheiden sich hinsichtlich langfristiger gesundheitsbezogener Maße und psychologischer Variablen. Für die Kontrollgruppe wird hierbei keine bedeutsame Veränderung erwartet. Für die Experimentalgruppen wird eine Verbesserung gesundheitsbezogener Maße (z.B. geringere Anzahl der Krankheitstage) und ein verbessertes Ausmaß emotionsbezogener Maße (z.B. geringeres Ausmaß an Depressivität) erwartet.

3) Die Gruppen unterscheiden sich in Bezug auf die langfristigen Auswirkungen auf Normorientierung, Verantwortungsübernahme und der Führung während der Haftzeit. Hierbei wird angenommen, dass sich eine Verbesserung dieser Maße in der Experimentalgruppe der „,Tataufarbeitung“ zeigt. 
4) Es wird angenommen, dass sich in den beiden Experimentalgruppen die Merkmale Alexithymie, Antisoziale Persönlichkeitsstörung sowie ausgewählte Essaymerkmale auf die langfristigen Ergebnisparameter auswirken.

\section{Methode}

\subsection{Design der Studie}

In Anlehnung an das klassische Schreibexperiment von Pennebaker et al. (1986) sind Design und Durchführung dieser Untersuchung so weit wie möglich ähnlich gestaltet. Das ES wird in zwei Bedingungsvariationen durchgeführt: eine Schreibgruppe schreibt über ein belastendes Lebensereignis, eine weitere über vergangene Straftaten. Die Kontrollgruppe schreibt über ein neutrales Thema (,trivial writing").

Die Schreibinstruktion wird unter Berücksichtigung des Klientels im Jugendstrafvollzug im Vergleich zur Originalstudie deutlich verkürzt und im Ausdruck vereinfacht. Um eine Strukturierung der Schreibaufgabe zu induzieren, werden die Teilnehmer der Experimentalgruppen gebeten, sich an den drei Schreibtagen auf verschiedene Aspekte des Erlebnisses zu konzentrieren (vgl. 2.2.4). Am ersten Schreibtag erhalten die Teilnehmer der Gruppe BE (belastendes Ereignis) eine zum klassischen Schreibparadigma analoge Instruktion. Sie sollen über ein persönliches Erlebnis schreiben, das in ihrem Leben bisher am „belastendsten oder schlimmsten“ für sie war. Dabei sollen sie ihre „tiefsten Gedanken, Gefühle und Empfindungen erkunden und ausdrücken“. Gleiches gilt für die Gruppe TA (Tataufarbeitung), die auf diese Weise über ihre vergangene Straftat schreiben soll. Am zweiten Schreibtag erhalten beiden Gruppen die Aufgabe, über die Zeit nach dem Ereignis und die Folgen für die Person selbst und andere Betroffene auf expressive Weise zu schreiben. Am dritten Schreibtag sollen sie sich das jeweilige Ereignis noch einmal vor Augen führen, um das Erlebte ,,abschließend für sich zu bewerten“ und ihre „heutige Sicht“ zu beschreiben. Die Teilnehmer der Gruppe BE werden zusätzlich instruiert, nicht über eine vergangene Straftat zu schreiben, auch wenn diese möglicherweise ein sehr belastendes Erlebnis für sie gewesen sein kann. In diesem Fall werden sie aufgefordert, über ein anderes Erlebnis aus ihrem Leben, das ebenfalls belastend für sie (gewesen) ist, zu schreiben. Die Teilnehmer der Kontrollgruppe (KG) sollen zunächst beschreiben, was sie am Vortag vom Aufstehen bis zum Zubettgehen getan haben. Am zweiten Tag 
schreiben sie darüber, was sie am selben Tag bis zur Schreibsitzung gemacht haben, am dritten Tag beschreiben sie, was sie am Folgetag tun werden. Dabei sollen sie so „objektiv und detailliert wie möglich“" ohne jeglichen Ausdruck eigener Gedanken und Gefühle schreiben (Anhang A5-A7).

\subsubsection{Versuchsplanung}

Dieser Untersuchung liegt ein zweifaktorielles Kontrollgruppendesign mit Messwiederholung zugrunde.

Der Faktor Bedingung ist dreifach gestuft: In einer Experimentalgruppe schreiben die Teilnehmer an drei aufeinander folgenden Tagen über ein persönlich belastendes Ereignis, das sie selber inhaltlich bestimmen (Gruppe BE), in der zweiten Gruppe schreiben die Teilnehmer über eine bzw. mehrere ihrer Straftaten (Gruppe TA). Beide Gruppen werden dabei aufgefordert, ihre tiefsten Gedanken und Gefühle auszudrücken. In der Kontrollgruppe wird unter den gleichen Bedingungen über ein neutrales Thema aus dem Bereich persönlicher Zeitplanung geschrieben (Gruppe KG). Die Teilnehmer der KG werden gebeten, so detailliert wie möglich zu schreiben. Sie werden instruiert, objektiv ohne den Ausdruck eigener Gefühle oder Meinung zu schreiben.

Der Messwiederholungsfaktor ist bezüglich der Überprüfung langfristiger Effekte des Schreibens zweifach gestuft: die Abhängigen Variablen werden im Rahmen der Erstuntersuchung vor der Schreibintervention erhoben und acht Wochen nach Beendigung der Intervention.

\begin{tabular}{|c|c|c|}
\hline \multirow{2}{*}{$\begin{array}{c}\text { Faktor } \\
\text { BEDINGUNG }\end{array}$} & \multicolumn{2}{|c|}{$\begin{array}{c}\text { Faktor } \\
\text { MESSWIEDERHOLUNG }\end{array}$} \\
\hline & $\begin{array}{c}\text { vor } \\
\text { Intervention }\end{array}$ & $\begin{array}{c}\text { acht Wochen } \\
\text { nach Intervention }\end{array}$ \\
\hline Belast. Ereignis & & \\
\hline Tataufarbeitung & & \\
\hline $\begin{array}{l}\text { Kontrollgruppe } \\
\text { Neutrales Thema }\end{array}$ & & \\
\hline
\end{tabular}

Abbildung 1: Untersuchungsdesign 
Als abhängige Variablen werden langfristige Veränderungen (acht Wochen nach der letzten Schreibsitzung) von körperlicher und psychischer Gesundheit, Emotionsregulation, Einstellungen zu Tat und Normen und die Führung während der Haft erhoben (vgl. auch 3.3).

Zur Überprüfung des Vorliegens von Moderatorvariablen werden Zusammenhänge zwischen Persönlichkeitseigenschaften und Essaymerkmalen einerseits und den langfristigen Effekten des Schreibens auf die verschiedenen abhängigen Variablen andererseits untersucht. So soll überprüft werden, ob das Vorliegen einer Antisozialen Persönlichkeitsstörung Einfluss auf Maße antisozialer Einstellung und Führung hat. Ferner wird ein möglicher Zusammenhang zwischen dem Ausmaß von Alexithymie und den langfristigen Veränderungen körperlicher und psychischer Gesundheit geprüft. Ausgewählte Essaymerkmale wie z.B. die Anzahl positiver bzw. negativer Emotionsworte werden ebenfalls zu diesen Maßen in Bezug gesetzt.

Bei der Überprüfung der Hypothesen zu kurzfristigen Effekten des Schreibens besitzt der Messwiederholungsfaktor zwei Stufen (unmittelbar vor und nach dem Schreiben) bzw. drei Stufen bei Vergleichen zwischen den drei Schreibtagen. Die kurzfristigen Schreibeffekte werden dabei als Überprüfung der experimentellen Manipulation genutzt. Hierbei werden stimmungsbezogene Reaktionen sowie die postexplorative Einschätzung der Probanden mittels Fragebögen erhoben.

\begin{tabular}{|l|c|c|}
\hline & 1. Schreibtag & 2. Schreibtag 3. Schreibtag \\
\hline Vor dem Schreiben & Stimmungsdiagnostik \\
\hline Nach dem Schreiben & $\begin{array}{c}\text { Stimmungsdiagnostik } \\
\text { Postexplorative Angaben }\end{array}$ \\
\hline
\end{tabular}

Abbildung 2: Darstellung des Manipulation Check

\subsubsection{Durchführung}

Die Untersuchung wird in den Räumlichkeiten der JVA Rosdorf (Abteilung Offener Jugendstrafvollzug) durchgeführt. Zunächst wird jeder Teilnehmer im Rahmen eines Erstgespräches detailliert über den Ablauf des Projekts, den Aufwand der Teilnahme und die datenschutzrechtlichen Bestimmungen aufgeklärt. Ihm wird außerdem erklärt, dass er möglicherweise über ein sehr persönliches Thema schreiben soll, dass eventuell sehr belastend für ihn gewe- 
sen ist. Ferner wird betont, dass es sich beim Schreiben nicht um einen Leistungstest handelt und Rechtschreib- und Grammatikfehler für die Auswertung und das Ergebnis der Studie völlig belanglos seien. Danach werden die Teilnehmer aufgefordert, ihr schriftliches Einverständnis (Anhang A2) zu geben und ihnen wird Vertraulichkeit im Umgang mit ihren Daten zugesichert. ${ }^{2}$ Im Fragebogen „Personenangaben“ werden Daten zu Herkunft, Familie, Inhaftierung, Tat und sozialer Einbindung erfasst (Anhang B1). Mittels strukturiertem Interviewleitfaden des SKID-II wird das Vorliegen einer Antisozialen Persönlichkeitsstörung erfasst. Im Fragebogen „Gesundheit“ werden Krankheitstage, Arztbesuche und Alkohol- und Drogenkonsum der letzten acht Wochen erfragt (Anhang B3). Im Anschluss füllen die Probanden nach kurzer mündlicher Instruktion alle weiteren Fragebögen aus. Diese erfassen im einzelnen körperliche Beschwerden, Affektivität, Angst, Ärger, Alexithymie und antisoziale Einstellung bzw. Normorientierung (vgl. 3.3). Schließlich werden Schreibtermine für drei aufeinander folgende Tage vereinbart.

Direkt vor der ersten Schreibsitzung wird der Teilnehmer per Zufall (Losziehung aus drei Alternativen) einer der drei Schreibbedingungen zugewiesen und erhält die entsprechende Instruktion. Nach zwei Monaten wird ein erneuter Termin zur Folgeuntersuchung gemacht.

Die teilnehmenden Inhaftierten erhalten mit Abschluss der Folgeuntersuchung eine Aufwandsentschädigung von $10 €$ durch Überweisung auf ihr Entgeltkonto .

Die drei Schreibsitzungen finden in einem Raum eines Wohngruppenhauses in der offenen Jugendanstalt statt. Dieser wird gewöhnlich für Mitarbeiterkonferenzen gebraucht und bietet die notwendige Abgeschiedenheit zur Durchführung eines Schreibexperimentes. Die Schreibinstruktion wird jedem Teilnehmer vorgelesen, er hat die Möglichkeit Fragen zu stellen und bekommt eine verkürzte schriftliche Instruktion vorgelegt, die die Kernaussagen enthält (Anhang A1). Dem Teilnehmer wird danach die Möglichkeit gegeben, allein und ohne Störungen 20 Minuten lang zu schreiben. Nach Ablauf der Zeit wird kurz an die Tür geklopft, so dass der Teilnehmer weiß, dass die Schreibzeit zu Ende ist. Er kann den begonnenen Satz noch zu Ende schreiben und schließlich sein Geschriebenes in einen bereitgelegten Umschlag geben und diesen in den dafür bestimmten Karton einwerfen.

Im Anschluss an jede Schreibsitzung wie auch in der Folgeuntersuchung werden die Teilnehmer gebeten, die Auswirkungen des Schreibens subjektiv einzuschätzen. Der dazugehörige postexperimentelle Fragebogen wurde im Rahmen einer Studie von Linkemann (2001) entwickelt und für die Zwecke der geplanten Studie mit Jugendlichen leicht modifiziert und

\footnotetext{
${ }^{2}$ Bei minderjährigen Probanden wird zusätzlich das schriftliche Einverständnis des/der Erziehungsberichtigten eingeholt.
} 
um einige Items ergänzt (Anhang B15). Anhand einer siebenstufigen Likert-Skala sollen die Teilnehmer nach jeder Sitzung einschätzen, wie persönlich und belastend das Thema des Schreibens für sie gewesen ist, ob es ihnen schwer fiel, ihre Gefühle auszudrücken und ob sie bisher bewusst vermieden haben, über das Thema zu sprechen. Diese Angaben werden als Überprüfung der experimentellen Manipulation betrachtet. Zum Zeitpunkt der Folgeuntersuchung sollen die Teilnehmer zusätzlich Angaben machen, wie sie das Projekt im nachhinein bewerten, ob sie seitdem ein Tagebuch führen und haben die Gelegenheit, Anmerkungen zu machen (Anhang B16).

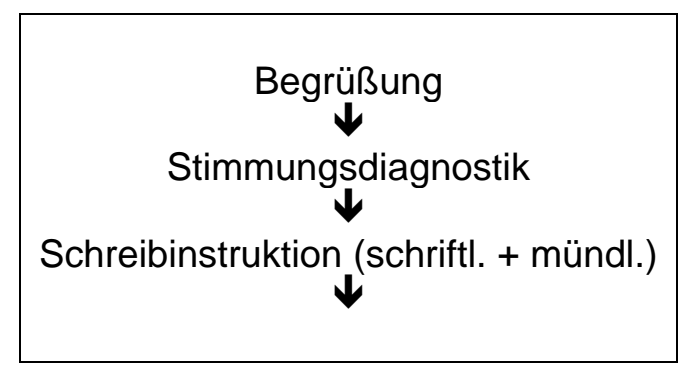

20 Minuten Schreiben

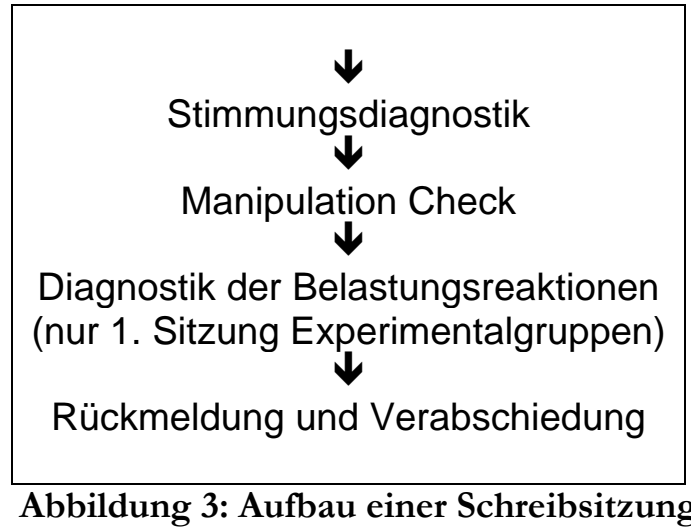

\subsubsection{Erhebungszeitpunkte}

Abbildung 4 ermöglicht einen Überblick über die Messzeitpunkte der Studie in Beziehung zum Haftverlauf der Teilnehmer. Neben den Erst- und Folgeuntersuchung wurden kurzfristige Stimmungsmaße direkt vor und nach dem Schreiben erhoben. 


\begin{tabular}{|c|c|c|c|c|c|}
\hline \multicolumn{6}{|c|}{ MONAT } \\
\hline 1 & 2 & 3 & 4 & 5 & 6 \\
\hline \multicolumn{3}{|c|}{$\begin{array}{l}\text { t } 0 \\
\text { Rekrutierung/ Ersterhebung } \\
\text { 3x Schreiben }\end{array}$} & \multicolumn{3}{|c|}{$\begin{array}{c}\mathrm{t} 1 \\
\text { Folgeuntersuchung }\end{array}$} \\
\hline & & & \multicolumn{3}{|c|}{$\begin{array}{l}\text { Erhebung der } \\
\text { Führungsbenotung }\end{array}$} \\
\hline \multirow{2}{*}{$\begin{array}{l}\text { Aufnahme des Inhaftier- } \\
\text { ten Zuweisung zu } \\
\text { Wohngruppe }\end{array}$} & \multirow{2}{*}{$\begin{array}{l}\text { erste mög- } \\
\text { liche Beno- } \\
\text { tung }\end{array}$} & Benotung & Benotung & Benotung & Benotung \\
\hline & & \multicolumn{4}{|c|}{$\begin{array}{l}\text { Anmerkung: Benotung beschreibt die Vergabe der Führungsbenotung } \\
\text { durch die Mitarbeiter der JVA }\end{array}$} \\
\hline
\end{tabular}

Abbildung 4: Messzeitpunkte im Haftverlauf

Die Maße der Führung jedes teilnehmenden Inhaftierten werden den Gefangenenakten der Haftanstalt entnommen und umfassen einen Verlauf von vier Monaten nach Beendigung der Teilnahme am Schreibprojekt. Sie werden demnach lediglich einmalig erfasst.

\subsection{Stichprobe}

Alle Inhaftierten des offenen Jugendvollzuges der JVA Rosdorf sind männliche Jugendliche im Alter von 14 bis 24 Jahren, die ihre Erststrafe verbüßen. Zumeist sind sie aufgrund von Eigentums- und Körperverletzungsdelikten zu einer Jugendstrafe verurteilt worden. Sie verweilen im Durchschnitt 11 Monate dort, bevor sie entlassen bzw. verlegt werden.

Zum Zeitpunkt der Erstuntersuchung sollen sich alle Teilnehmer nicht länger als 2 bis 3 Monate im Vollzug befinden, um so eine maximale Vergleichbarkeit des Fortschreitens vollzuglicher Maßnahmen und Behandlungen bei den Probanden sicherzustellen. Ein weiteres Auswahlkriterium der Teilnehmer liegt in ihrer Schreibtauglichkeit. Da sich alle Neuzugänge einem Schultest unterziehen müssen, ist aus den Akten ersichtlich, wer von ihnen voraussichtlich weniger Schwierigkeiten beim für die Intervention notwendigen Lesen und Schreiben haben wird.

Vom Projekt ausgeschlossen werden:

- Inhaftierte, die sich zum Zeitpunkt der Rekrutierung noch nicht bzw. bereits länger als 2 bzw. 3 Monate im Regelvollzug befinden, wobei eine vorangegangene Unterbringung in der Untersuchungshaft auf diese Zeitspanne nicht angerechnet wird, 
- Inhaftierte, die insgesamt weniger als 6 Monate im Vollzug bleiben (Haftzeit) und damit zur Folgeuntersuchung dort nicht mehr erreichbar sind,

- solche, die nicht ausreichend lese- und schreibtüchtig sind

- und Inhaftierte, deren Muttersprache nicht Deutsch ist.

Im Rahmen dieser Untersuchung werden 60 jugendliche und heranwachsende Inhaftierte im Alter von 16 bis 24 Jahren rekrutiert und nehmen am so genannten „Schreibprojekt“ teil. Die Inhaftierten werden zunächst im Rahmen der ersten vollzuglichen Maßnahme, dem Startkurs, von pädagogischen Mitarbeitern der JVA auf das Projekt angesprochen. Falls sie Interesse haben, am Projekt teilzunehmen, wird ein Termin zur Erstuntersuchung ausgemacht. Zu diesem Zeitpunkt wird verdeutlicht, dass es sich um eine freiwillige Teilnahme handelt, die unabhängig von jeglicher innervollzuglicher Behandlungs- und Stufenplanung ist. So wirkt sich eine Teilnahme nicht explizit positiv auf die Führungsbenotung der Inhaftierten aus.

Im Rahmen der Rekrutierung zeigt sich, dass sich Inhaftierte mit unzureichender Schreibkenntnis zumeist nicht bereit erklären, an der Studie teilzunehmen, wenn auch oft mit anderer Begründung. Insgesamt 12 Inhaftierte wollen auf Anfrage (durch Mitarbeiter der JVA oder Versuchsleiterin) nicht teilnehmen, weil sie „keine Lust“ hätten und/oder weil sie sich nicht mit Erlebnissen aus ihrer Vergangenheit auseinandersetzen wollten. Drei Teilnehmer fallen während des Projektes vor dem Zeitpunkt der Folgeuntersuchung aufgrund von Verlegung in eine andere Haftanstalt heraus. Um sie zu ersetzen, werden drei weitere Teilnehmer rekrutiert.

Die Gesamtstichprobe weist ein Durchschnittsalter von 19,7 Jahren $(\mathrm{SD}=1,72)$ auf und ist zum Zeitpunkt der Rekrutierung seit durchschnittlich 50 Tagen $(S D=50,73)$ inhaftiert und im Mittel seit 38 Tagen $(\mathrm{SD}=27,03$; Minimum=11, Maximum=150) in dieser Anstalt. Die Jugendlichen verbüßen eine Strafdauer von 15,1 Monaten (SD=7,70) mit einer Minimalstrafzeit von 6 und einer Maximalstrafzeit von 36 Monaten. Die Mehrheit von ihnen (88\%) gibt an, ledig zu sein. Knapp über die Hälfte (52\%) besitzt keinen Schulabschluss, 33\% haben einen Hauptschul- und 10\% einen Sonderschulabschluss. Ähnlich sieht es bezüglich der beruflichen Bildung aus: 58\% der Teilnehmer haben keine Berufsausbildung bzw. eine Lehre begonnen, 35\% haben sie zwar begonnen, aber nicht abgeschlossen.

Befragt nach ihrer Herkunft und Familie zeigt sich das folgende Bild: 37\% von ihnen haben im Heim gelebt (mit einer durchschnittlichen Dauer von 41 Monaten), bei 20\% ist ein Elternteil verstorben oder unbekannt, knapp über die Hälfte der Jugendlichen ist überwiegend mit 
beiden Elternteilen bzw. mit Mutter und Lebenspartner aufgewachsen. Zum ersten Mal straffällig wurden sie nach eigener Angabe mit 13,5 Jahren $(\mathrm{SD}=3,46)$. Dafür wurde die Hälfte von ihnen in irgendeiner Form sanktioniert.

Gefragt nach psychiatrischer bzw. psychotherapeutischer Vorbehandlung geben 17 Jugendliche an, hiermit bereits in Kontakt gewesen zu sein (vor durchschnittlich 5 Jahren). 5 Teilnehmer sind wegen Drogenproblemen behandelt worden.

Zum Zeitpunkt der Ersterhebung befinden sich 22 der 60 Teilnehmer in einer beruflichen bzw. schulischen Maßnahme der Anstalt, die anderen 38 Teilnehmer sind entweder noch im „Startkurs“ oder als Hausarbeiter (bei kurzer Strafzeit) beschäftigt. Drei Jugendliche befinden sich in einer therapeutischen Maßnahme. Auf die Frage nach chronischen Krankheiten geben 10 von ihnen an, derzeit ein chronischen Leiden zu haben (zumeist Asthma oder Heuschnupfen). In regelmäßiger Medikation befinden sich 3 Teilnehmer.

Ihre momentane Stimmung (angegeben auf einer Likert-Skala von $1=$ „,sehr gut“ bis $5=$,sehr schlecht") liegt im Durchschnitt bei 2,98 ( $\mathrm{SD}=0,87)$, die wahrgenommene Belastung durch die Inhaftierung bei 2,68 ( $\mathrm{SD}=0,89$; Skala von 1= ,gar nicht belastet" bis $4=$,,sehr belastet" $)$. Befragt nach möglichen belastenden Lebensereignissen der letzten drei Monate geben zwei Drittel der Stichprobe zum Ersterhebungszeitpunkt an, ein solches Ereignis erlebt zu haben, wobei hier überwiegend die Inhaftierung genannt wird.

Informationen zu den von den Teilnehmern der Studie begangenen Straftaten gibt Tabelle 4.

Tabelle 4

Merkmale der Straftaten

\begin{tabular}{|c|c|c|}
\hline & $\mathrm{n}$ & $\%$ \\
\hline \multicolumn{3}{|l|}{ Delikte (Mehrfachnennung möglich) } \\
\hline einfaches Delikt & 22 & 37 \\
\hline Mehrfachdelikte: & 48 & 80 \\
\hline Diebstahl & 18 & 30 \\
\hline Körperverletzung & 22 & 37 \\
\hline Betrug & 9 & 15 \\
\hline Einbruch (davon 7 kombiniert mit Diebstahl) & 8 & 13 \\
\hline Verstoß gegen Betäubungsmittelgesetze & 6 & 10 \\
\hline Sonstige wie Fahren ohne Führerschein, Sachbeschädigung & 8 & 13 \\
\hline \multicolumn{3}{|l|}{ Täterschaft } \\
\hline Einzeltäter & 26 & 44 \\
\hline Gruppentäter & 34 & 56 \\
\hline Noch ausstehende Verfahren & 19 & 31 \\
\hline
\end{tabular}




\begin{tabular}{|l|c|c|}
\hline Gründe für Straftaten nach eigener Angabe & 16 & 27 \\
(Mehrfachnennung möglich) & 16 & 27 \\
Geldsorgen/Schulden (als mind. ein Grund angegeben) & 14 & 23 \\
Alkohol- Drogeneinfluss zum Tatzeitpunkt & 9 & 15 \\
Einfluss der/des Mittäter (s) & 2 & 3 \\
Langeweile & & \\
Spaß & & \\
Schuld an der Begehung der Straftat(-en) hatte ... & 41 & 68 \\
nach eigener Angabe & 9 & 15 \\
$\ldots$ ich selbst & 7 & 12 \\
.. der andere & & \\
Drogir, die Täter & & \\
Alkohol & 28 & 47 \\
Haschisch & 12 & 20 \\
Verschiedene Substanzen (meist Alkohol und Haschisch) & 12 & 20 \\
keinen & 21 & 35 \\
Frühere Sanktionen & & \\
Bewährungsstrafe & & \\
Jugendarrest & 47 & 78 \\
Andere Sanktionen (z.B. Arbeitsauflagen, Geldbußen) & 37 & 62 \\
& 53 & 88 \\
\hline
\end{tabular}

\subsection{Abhängige Variablen}

\subsubsection{Körperliche und psychische Gesundheit}

\subsubsection{Krankheitstage, Arztbesuche und Substanzgebrauch}

Die Teilnehmer werden zum Zeitpunkt der Prä- und der Katamneseuntersuchung gebeten, bezogen auf die vergangenen acht Wochen Auskunft über folgende Gesundheitsaspekte zu geben (Anhang B2 ):

- Anzahl der Krankheitstage sowie kurze Beschreibung des Krankheitsgrunds,

- Anzahl der Arzt- und Zahnarztbesuche und kurze Beschreibung des Anlasses,

- Häufigkeit des Alkohol- und Drogenkonsums (Unterscheidung zw. Zeitraum vor und während der Haft ), ggf. Angabe der konsumierten Substanz.

Auf die Erhebung objektiver Daten über Arztakten wird verzichtet, da sich datenschutzrechtliche Probleme ergeben. Zudem wurde nach vorheriger Sichtung der Gefangenenakten deut- 
lich, dass, wenn überhaupt, sich in diesen lediglich in unsystematischer Weise Vermerke eines behandelnden Arztes wieder finden.

Was Angaben über den eigenen Alkohol- und Drogenkonsum betrifft, so ist nicht davon auszugehen, dass diese, bezogen auf die Haftzeit, in jedem Fall wahrheitsgemäß sind. Obwohl vor der Auskunft noch einmal betont wird, dass alle Daten vertraulich behandelt werden, ist nicht auszuschließen, dass viele Inhaftierte im Falle des Bekanntwerdens dieser Informationen negative Konsequenzen wie etwa die Verlegung in eine geschlossene Haftanstalt befürchten. Aus Gründen der vollständigen Exploration sollen diese Daten dennoch erhoben werden, da Alkohol- und Drogenkonsum im Leben inhaftierter Probanden eine wichtige Rolle spielen und sich auf ihr Befinden und ihre Gesundheit auswirken können.

\subsubsection{Körperliche Beschwerden}

Zur Messung langfristiger Veränderungen körperlicher Beschwerden wird als Selbstauskunftsmaß die Beschwerdenliste (v. Zerssen et al., 1976) eingesetzt (Anhang B7). Auf einer vierstufigen verbalen Skala (von „gar nicht“ bis „,stark“) wird für insgesamt 24 körperliche Beschwerden (z.B. Zittern, Mattigkeit) das Ausmaß subjektiver Beeinträchtigung eingeschätzt. Die Teilnehmer sollen sich bei dieser Beurteilung auf die vergangenen vier Wochen beziehen.

In Untersuchungen zu den Gütekriterien erwies sich die Beschwerdenliste mit Reliabilitätskoeffizienten zwischen .87 (Cronbach's $\alpha$ ) und .95 (Spearman-Brown) als reliabel (v. Zerssen, 1976). Die Skala wird explizit für den Gebrauch in Verlaufsuntersuchungen empfohlen. Für die Auswertung werden den Antwortskalen Zahlenwerte ( $0=$ gar nicht bis $3=$ stark) zugeordnet und anschließend wird der Mittelwert über alle Items als Gesamtscore körperlicher bzw. psychosomatischer Beschwerden berechnet.

\subsubsection{Psychische Symptombelastung}

Zur Erfassung langfristiger Effekte auf die psychische Symptombelastung wird die Kurzform der Symptomcheckliste SCL-90-R von Derogatis (1977) verwendet. Bei der SCL-K-9 (Klaghofer \& Brähler, 2000) handelt es sich wie bei der ursprünglichen Langform um ein Selbstbeurteilungsinstrument mit einer 5-Punkt-Antwortmöglichkeit (Anhang B4). Die SCL-K-9 stellt eine eindimensionale Kurzversion dar, bei deren Konstruktion anhand einer Repräsentativbefragung aus jeder der ursprünglich 9 Skalen der SCL-90-R dasjenige Item mit der höchsten Korrelation zum Global Severity Index (GSI-90) ausgewählt wurde. Damit besteht die Skala aus lediglich 9 Items und korreliert mit dem GSI-90 zu r=.93. Jedes Item repräsentiert eine 
der Skalen des SCL-90-R, nämlich Somatisierung, Zwanghaftigkeit, Unsicherheit im Sozialkontakt, Depressivität, Ängstlichkeit, Aggressivität/Feindseligkeit, Phobische Angst, Paranoides Denken und Psychotizismus. Hinsichtlich der Testgütekriterien zeigt sich die SCL-K-9 intern konsistent und reliabel (Cronbach`s $\alpha=.87$ ) und zeigt bedeutsame Korrelationen mit beispielsweise den Skalen des Giessener Beschwerdefragebogens (GBB) und der der Hospital Anxiety and Depression Scale (HAD-S). Für die Auswertung wird in Anlehnung an die Langversion der Globalwert GSI-9 errechnet. Die Skala empfiehlt sich zum Screening in der klinischen Diagnostik und wurde in dieser Studie auch aufgrund ihrer Ökonomie eingesetzt.

\subsubsection{Belastungsreaktionen}

Das Ausmaß der durch das beschriebene Erlebnis hervorgerufene Belastungsreaktionen wird mittels der Impact of Event-Skala- revidierte Form IES-R (Weiss \& Marmar, 1996) erhoben. Es wird die von Maercker \& Schützwohl (1998) bearbeitete und ins Deutsche übersetzte Version der IES-R eingesetzt (Anhang B9). Insgesamt 22 Items erfassen drei Formen typischer psychischer Reaktionen auf Extremereignisse, die sich drei Skalen zuordnen lassen:

1. Intrusionen: die Items dieser Subskala beziehen sich auf aufdrängende, belastende Erinnerungen, unkontrollierbare Gedanken, Bilder, Träume und Gefühle;

Bsp. „Immer, wenn ich an das Ereignis erinnert wurde, kehrten die Gefühle wieder.“

2. Vermeidung: diese Subskala erfasst Anstrengungen, solche Gedanken, Gefühle, Aktivitäten oder Situationen zu vermeiden, die mit dem Trauma in Verbindung stehen und die Erinnerung daran wachrufen;

Bsp. „Ich versuchte, Erinnerungen daran aus dem Weg zu gehen.“

3. Übererregung: Übererregungssymptome umfassen Störungen des Schlafs oder der Konzentration, erhöhte Reizbarkeit, Vigilanz sowie übertriebene Schreckreaktionen;

Bsp. „Ich war leicht reizbar und schreckhaft.“

Die Subskalen „Intrusionen“ und „Überregung“ enthalten jeweils 8, die der Vermeidung 7 als Selbstaussagen formulierte Items. Die Auftretenshäufigkeit der Belastungsreaktionen wird anhand einer vierstufigen verbalen Skala von „überhaupt nicht“ bis „oft“ eingeschätzt.

Die interne Konsistenz der deutschen Version der IES-R variiert bei den verschiedenen Skalen zwischen .79 für die Skala „Vermeidung“ und .90 (Cronbach’s $\alpha$ ) für die Skalen „Intrusionen“ und „Übererregung“ (Maercker \& Schützwohl, 1998). Für die Auswertung werden den Skalenkategorien Zahlenwerte zugeordnet und anschließend für jede Subskala ein mittlerer Itemwert berechnet. Ergibt sich durch Formelberechnung ein resultierender Wert $>0,0$ ist die 
Wahrscheinlichkeit des Vorliegens einer Posttraumatischen Belastungsreaktion wahrscheinlich.

Die IES-R wird in dieser Untersuchung lediglich den beiden Experimentalgruppen (BE, TA) zur Beantwortung vorgelegt, da sich alle Items auf ein zuvor festgelegtes (belastendes) Ereignis beziehen. In diesem Fall auf jenes, über das geschrieben werden soll. Die Kontrollgruppe unterscheidet sich - dem Vorgehen Pennebakers entsprechend - gerade durch das Fehlen der Auseinandersetzung mit einer belastenden Erfahrung. Die Bearbeitung der Belastungsfragebögen mit ihrem Bezug zu einem belastenden Ereignis hätte möglicherweise in der Kontrollgruppe die Auseinandersetzung mit einem solchen mit sich gebracht und eventuell zu einer Verzerrung der Ergebnisse geführt.

\subsubsection{Maße der Emotionsregulation}

\subsubsection{Positiver und negativer Affekt (kurz- und langfristig)}

Kurz- wie auch langfristige Effekte des Schreibens auf die Affektivität werden mit Hilfe der Positive and Negative Affect Schedule (PANAS) von Watson, Clark \& Tellegen (1988) erhoben. Dieser Fragebogen basiert auf dem Zwei-Faktoren-Modell der Affektivität von Watson und Tellegen (1985), das positiven und negativen Affekt als zwei voneinander unabhängige Grunddimensionen der Affektivität auffasst. Die PANAS umfasst 20 Adjektive der Stimmungsbeschreibung, die jeweils zur Hälfte positive (z.B. stark, interessiert, begeistert) und negative (z.B. traurig, beschämt, durcheinander) Gefühle und Empfindungen beschreiben. Diese werden anhand einer fünfstufigen Likert-Skala von 1= ,ganz wenig oder gar nicht“ bis $5=$, ,sehr“ eingeschätzt.

In dieser Untersuchung wird die von Krohne, Egloff, Kohlmann und Tausch (1996) ins Deutsche übersetzte Version der PANAS eingesetzt (Anhang B8). Das ursprüngliche Adjektiv „bekümmert“ wurde aus Zwecken der Verständlichkeit durch das Adjektiv „traurig“ ersetzt, da erwartet werden konnte, dass viele der Teilnehmer das Wort „bekümmert“" weder verwenden noch verstehen.

Je nach Untersuchungsplan kann die Skala mit (bis $\mathrm{zu}$ sechs) verschiedenen Instruktionen vorgelegt werden, die sich auf Angaben zu unterschiedlich erstreckten Zeitintervallen beziehen. Zur Erfassung langfristiger bzw. habitueller Affektivität (Zeitpunkt der Erst- und Folgeuntersuchung) lautet die Instruktion: „Wie fühlen sie sich im allgemeinen?“ und zur Messung kurzfristiger Veränderungen durch das Schreiben (direkt vor und nach jeder Schreibsitzung) wird die Instruktion: „Wie fühlen Sie sich im Moment?“ eingesetzt. 
Die PANAS weist eine interne Konsistenz (Cronbach's $\alpha$ ) von .84 (positiver Affekt) bzw. von .86 (negativer Affekt) auf und eine für den jeweiligen momentanen Affekt erwartungsgemäß niedrige Retest-Reliabilität von .19. Der Retest-Realibilitätskennwert für die habituelle Affektivität fällt mit .66 (positive Affektivität) bzw. von .54 (negative Affektivität) hingegen höher aus, ist allerdings niedriger ausgeprägt als die internen Konsistenzen. Dies wird damit erklärt, dass die selbstbeurteilte habituelle Affektivität vermutlich auch durch die aktuelle Stimmungslage beeinflusst wird (Krohne et al., 1996).

Bei der Auswertung wird für jede Skala getrennt der Mittelwert als Gesamtindex positiver bzw. negativer Affektivität (PA und NA) ermittelt.

\subsubsection{Depressivität}

Mittels der Kurzform der Allgemeinen Depressionsskala (ADS-K) von Hautzinger \& Bailer (1993a) werden langfristige Effekte des Schreibens auf die depressive Symptomatik erfasst werden. Bei der ADS-K handelt es sich um ein Selbstbeurteilungsinstrument, das das Vorhandensein und die Dauer der Beeinträchtigung durch depressive Affekte, körperliche Beschwerden, motorische Hemmung und negative Denkmuster erfragt. Die ADS-K umfasst 15 Items, die Merkmale wie z.B. Verunsicherung, Erschöpfung, Hoffnungslosigkeit und Selbstabwertung abfragen (Anhang B5). Die innere Konsistenz der Skala liegt bei .90, sie korreliert mit der Befindlichkeitsskala BL (von Zerssen, 1986) mit .73 ( $\mathrm{p}<.001)$ und mit dem Inventar depressiver Symptome IDS (Hautzinger \& Bailer, 1993b), einem Fremdbeurteilungsinstrument depressiver Symptome mit $.67(\mathrm{p}<.001)$.

Der Bezugszeitraum der ADS-K ist die vergangene Woche. Bei der Auswertung wird der Summenwert der Antworten als Kennwert aktueller depressiver Symptomatik verwendet. Es ist möglich, als „,unglaubwürdig“ einzustufende Skalenantworten über das so genannte „Lügenkriterium“ zu berechnen. Wird ein kritischer Wert von 24 Punkten erreicht, sollten Fragebogenantworten als nicht glaubwürdig angesehen werden. Bei der hier untersuchten Stichprobe ist die Bestimmung des Lügenwertes angezeigt, da sozial erwünschte Antworttendenzen zu erwarten sind.

\subsubsection{Zustandsangst und -ärger}

Mittels State-Trait-Angstinventars (STAI), das ursprünglich von Spielberger, Gorsuch \& Lushene (1970) entwickelt wurde und in einer deutschen Version vorliegt (Laux, Glanzmann, Schaffner \& Spielberger, 1981) können Zustandsangst und Angst als Eigenschaft bzw. Ängstlichkeit getrennt erfasst werden. Die Angstskala stützt sich auf zwanzig Feststellungen, mit 
denen der Proband beschreiben soll, wie er sich jetzt in diesem Moment bzw. wie er sich im allgemeinen fühlt. Da das Hauptaugenmerk hier auf variablen Maßen der Emotionsregulation liegt und diese mit änderungssensitiven Messinstrumenten zu erheben sind, wird in dieser Studie lediglich die Zustandsangst (Form X1 des STAI) der Probanden erfasst (Anhang B10). Die State-Angstskala gibt ein Maß für die Intensität eines emotionalen Zustands, der gekennzeichnet ist durch Anspannung, Besorgtheit, Nervosität, innere Unruhe und Furcht vor zukünftigen Ereignissen. Die Skala kann in experimentellen wie auch in „real life“- Situationen eingesetzt werden, um Angst in Abhängigkeit von internen und externen Einflüssen zu bestimmen.

Die innere Konsistenz liegt für die State-Angstskala bei $r=.90$ und ihre Retest-Reliabilität (Retestintervall von 63 Tagen) erwartungsgemäß niedrig bei $r=.22$ bis $r=.53$. Die niedrige Retest-Reliabilität entspricht der Konzeption der Zustandsangst im Sinne eines zeitlich instabilen fluktuierenden Merkmals. Bei der Auswertung wird ein Summenwert (von 20 bis 80) bestimmt.

Ärger als eine weitere grundlegende Emotion lässt sich ebenfalls in der Tradition von TraitState-Dimensionen mittels des State-Trait-Ärgerausdrucks-Inventars (STAXI) (Spielberger, 1988; deutsche Fassung von Schwenkmezger, Hodapp \& Spielberger, 1992) erfassen. Ärger als Zustand, der im Rahmen dieser Studie untersucht wird, ist definiert als emotionales Befinden, das aus subjektiven Gefühlen der Spannung, Störung, Irritation und Wut besteht und von einer Aktivierung des autonomen Nervensystems begleitet wird. Der Ärger-Zustand kann hinsichtlich Intensität und als Funktion des Anstiegs von Frustration variieren, die aus wahrgenommener Ungerechtigkeit oder Blockierung zielgerichteter Verhaltensweisen resultiert (vgl. ebd.).

Die Ärger-Zustandsskala des STAXI (State-Anger; S-A) umfasst zehn Selbstbeschreibungsitems, die auf einer vierstufigen Intensitätsskala (von 1=, überhaupt nicht“ bis 4=,,sehr“) hinsichtlich des augenblicklichen Gefühlszustands beurteilt werden sollen (Bsp. „Ich bin ungehalten. “; Anhang B6). Die innere Konsistenz der Teilskalen liegt zwischen r=.71 und $\mathrm{r}=.95$. Die Werte der Retestreliabilität sind insgesamt uneinheitlich (zwischen .60 und .20), aber erwartungsgemäß niedriger als die Koeffizienten der Eigenschafts-Ärgerskalen. Die Testauswertung erfolgt über Addition von Punktwerten pro Item jeder Skala. 


\subsubsection{Maße der Normorientierung und Führung}

Legalprognostisch relevante Daten werden im Rahmen dieser Studie sowohl im Selbst- wie auch im Fremdbericht erhoben. Sie werden über Fragebögen und die Gefangenenakten der Haftanstalt erfasst.

\subsubsection{Normorientierung und Verantwortungsübernahme}

Um das Ausmaß nonkonformer bzw. antisozialer Einstellung, Werte und Denkmuster zu bestimmen, werden ausgewählte Subskalen der Criminal Sentiments Scale - modified CSSM (Simourd, 1997) eingesetzt. Diese wurde nach Absprache mit dem Autor David Simourd zunächst ins Deutsche übersetzt und danach von einem englischen Muttersprachler ins Englische rückübertragen, um etwaige Abweichungen zwischen Original und der deutschen Übersetzung in der Erstfassung zu korrigieren. Bei den in dieser Studie eingesetzten Subskalen der CSS-M handelt es sich um die Skalen „Tolerance of law violation“ (TLV) und „Identification with criminal others" (ICO).

Die TLV-Subskala beinhaltet Aussagen, die Neutralisierungstendenzen kriminellen Verhaltens reflektieren.

Bsp. „Jemand wie ich muss manchmal das Gesetz brechen, um im Leben vorwärts zu kommen. “

Die Items der ICO-Subskala stellen wertende Urteile bezüglich anderer Straffälliger dar Bsp. „Leute, die das Gesetz gebrochen haben, haben die gleichen Einstellungen zum Leben wie ich. “

Beide Skalen beinhalten insgesamt 16 Items, die jeweils Einstellungen beschreiben, denen der Proband auf einer dreistufigen Antwortskala zustimmt bzw. nicht zustimmt oder sich ihr unentschlossen gegenüber zeigt (Anhang B11).

Die (englische Originalversion der) CSS-M weist eine interne Konsistenz von .75 (Cronbach's $\alpha$ ) auf. Untersuchungen zur Kriteriumsvalidität bei Inhaftierten zeigen Korrelationen mit verschiedenen Rückfälligkeitsvariablen wie Führungsaufsichtsverletzungen, erneute Festnahme und Inhaftierung in einem Zeitraum bis zu einem Jahr nach der Entlassung (Simourd \& van de Ven, 1999).

Für die Auswertung werden den Items der Subskalen Zahlenwerte zugeordnet, die anschließend zu einem Gesamtscore addiert werden. 
Mittels eines weiteren Fragebogens sollen Veränderungen in der Einschätzung der Folgen von Straftaten erfasst werden. Die Teilnehmer werden gebeten, einzuschätzen, inwiefern es sich lohnt, Straftaten zu begehen bzw. inwieweit es von Vorteil ist, gesetzeskonform zu leben und somit keine Straftaten zu begehen. Die Beurteilung von Folgen von Straftaten kann auch als „moralisches Urteil“ bezeichnet werden und wird über den vom Kriminologischen Forschungsinstitut Niedersachsen (Hosser \& Greve, 1999) entwickelten Fragebogen (hier MUBFS genannt= Moralisches Urteil- Beurteilung der Folgen von Straftaten), erfasst (Anhang B13). ${ }^{3}$

Der Fragebogen besteht aus zwei Teilen: auf einer vierstufigen Skala (von „trifft nicht zu“ bis „trifft völlig zu“) werden zum einen acht Aussagen beurteilt, warum es sich lohnen könnte, Straftaten zu begehen.

\section{Bsp. „Es lohnt sich Straftaten zu begehen, weil der Gewinn höher ist als die} Strafe“.

Zum anderen werden acht dazu äquivalente Aussagen eingeschätzt, warum es sich eventuell nicht lohnt, Straftaten zu begehen.

\section{Bsp. „Straftaten sollte man besser nicht begehen, weil der Gewinn die Strafe niemals ausgleicht“.}

Die Auswertung erfolgt über die Summenbildung der Zahlenwerte (von 8 bis 32) der Items für jede Skala.

Um das Ausmaß von Verantwortungsübernahme bzw. Verantwortungsablehnung der Teilnehmer bezüglich ihrer Straffälligkeit zu erfassen, wird der (in dieser Studie so benannte) „Berner Fragebogen“ (Oswald \& Bütikofer, 2003a) eingesetzt, der Aussagen insbesondere bezogen auf die eigenen Straftaten enthält. ${ }^{4}$ Der Fragebogen enthält 30 Items, von denen 8 aus einem Vorgängerverfahren (Prisonisierungsfragebogen von Ortmann, 1987) stammen. Auf einer vierstufigen Likert-Skala (von 1= „stimmt nicht" bis 4=,,stimmt") sollen die Probanden für jede Aussage das Ausmaß ihrer Zustimmung angeben (Anhang B12).

Faktorenanalytisch ergaben sich bei einer Erstüberprüfung des Fragebogens ein Rechtfertigungs- und ein Entschuldigungsfaktor. Dabei spiegelt die Entschuldigung eine Strategie wider, mit der die Person die Norm anerkennt, sich aber von der Verantwortlichkeit zu entbin-

\footnotetext{
${ }^{3}$ Das Kriminologische Forschungsinstitut Niedersachsen (KFN) entwickelte diesen Fragebogen als Teil eines Interviews, das im Rahmen des Forschungsprojekts „Gefängnis und die Folgen - Identitätsentwicklung und kriminelles Handeln während und nach Verbüßung einer Jugendstrafe" bei bisher 2400 jugendlichen und heranwachsenden Inhaftierten eingesetzt wurde.

${ }^{4}$ Im Rahmen des Modellversuches „Tataufarbeitung und Wiedergutmachung“ entwickelte die Forschergruppe der Universität Bern verschiedene Fragebögen, u.a. einen zur Einstellung zum Delikt, der im folgenden als „Berner Fragebogen" bezeichnet wird.
} 
den versucht. Umgekehrt stellt die Rechtfertigung eine Strategie dar, mit der die Person die Verantwortlichkeit für die Tat übernimmt, sie jedoch nicht als Normverletzung akzeptiert (Oswald \& Bütikofer, 2003b).

Beispiel-Items:

„Ich hatte wirklich keine Ahnung, was ich durch meine Tat bei anderen angerichtet habe. “(Entschuldigung)

„Was ich getan habe, tun heutzutage so viele, dass es auf einen mehr oder weniger gar nicht mehr ankommt. “ (Rechtfertigung)

Die Auswertung des Fragebogens erfolgt unter der Berücksichtigung zweier umgekehrt gepolter Items über die Summierung der Zahlenwerte der Items der drei Subskalen (Rechtfertigung, Entschuldigung, Items des Prisionierungsfragebogens von R. Ortmann) und der Berechnung eines Gesamtscores.

\subsubsection{Führung während der Haftzeit}

Als verhaltensnahes Maß wird zusätzlich von jedem Inhaftierten die monatliche Benotung der Führung und damit verbundener so genannter Stufung den Akten entnommen (vgl. Anhang B17). Bei der Benotung werden Leistungs- und Sozialverhalten der Inhaftierten beurteilt. Diese monatliche Beurteilung (von 1="sehr gut" bis 6="sehr schlecht") wird in den Bereichen Schule/Arbeit, Wohn-/Erziehungsgruppe und Sport/Freizeit von verschiedenen Bediensteten der JVA vergeben und schließlich zu einer Gesamtnote zusammengezogen, wobei die schlechteste Note als Gesamtnote gewertet wird.

Aus dieser Einschätzung des Führungsverhaltens wird unter Berücksichtigung der abgeleisteten Haftzeit die Stufung des Inhaftierten abgeleitet. Die Stufen (von 0 bis 4) entsprechen dabei in etwa der Anzahl der Monate, die ein Inhaftierter bereits im Vollzug abgeleistet hat. Jede Stufe erfordert eine Mindestnote, um Vollzugslockerungen wie z.B. Stadtausgänge bewilligt zu bekommen. Bei guter Benotung ist es möglich, höher gestuft zu werden, bei schlechter Note kann Stufenverlängerung oder Rückstufung drohen. Im Erhebungsverlauf dieser Studie werden maximal 4 bis 5 (monatliche) Benotungen pro Teilnehmer berücksichtigt, aus denen wiederum eine Durchschnittsnote gebildet wird.

Aus den Gefangenenakten kann ferner entnommen werden, ob es in Einzelfällen zu Regelverstößen gekommen ist. In diesen Fällen kommt es zu Anhörungen, in denen der Inhaftier- 
te zum jeweiligen Vorfall (z.B. unerlaubten Alkoholkonsum) befragt und ihm die Möglichkeit gegeben wird, sich zu äußern. Diese Anhörungen werden in den Akten als Anhörung(svermerke) geführt und geben ferner Auskunft über mögliche daraus resultierende Sanktionen (z.B. zusätzliche Arbeitsstunden). Zudem werden alle sonstigen Vermerke aus den Akten, die Aufschluss über Fehlverhalten des Inhaftierten (z.B. Arbeitsverweigerung, unerlaubter Handybesitz) geben, gelistet. Bei vielen Vorfällen kommt es zur Eintragung von sowohl Vermerken wie auch Anhörungen. Akteneinträge zum gleichen Vorfall werden lediglich einfach als Anhörung gezählt. Einträge, bei denen den Inhaftierten im engeren Sinne keine Schuld trifft, er beispielsweise von einem Mithäftling körperlich angegriffen wurde und eine Aussage hierzu tätigte, werden ebenfalls nicht berücksichtigt. Die Anhörungen und Vermerke werden nach zeitlichem Auftreten notiert und schließlich summiert. ${ }^{5}$

\subsection{Moderatorvariablen}

\subsubsection{Alexithymie}

Der Begriff Alexithymie wurde zur Beschreibung eines Persönlichkeitsaspekts geprägt, das charakterisiert ist durch „die Schwierigkeit, Gefühle zu identifizieren und von körperlichen Sensationen zu unterscheiden, die Schwierigkeit, Gefühle auszudrücken und eine konkretistische, extern orientierte Denk- und Sprechweise, die nur geringe Verbindungen zu affektiven Komponenten und einen Mangel an Phantasie“ aufweist (vgl. Bach, Bach, de Zwann, Serim \& Böhmer, 1996, S. 23). Zur Erfassung von Alexithymie wird die deutsche Fassung der Toronto Alexithymia Scale (TAS-20; Bagby, Parker \& Taylor, 1994) von Bach et al. (1996) in der revidierten 20-Item-Version eingesetzt. In diesem Selbstbeurteilungsverfahren trifft der Proband seine Einschätzung auf einer fünfstufigen Skala, die von ,trifft überhaupt nicht $\mathrm{zu}^{\text {“ }}$ bis ,trifft immer zu“ reicht (Anhang B3).

Beispiel-Items sind:

„Wenn ich aufgeregt bin, weiß ich oft nicht, ob ich traurig, ängstlich oder wütend bin.“

„Es fällt mir schwer, die richtigen Worte für meine Gefühle zu finden.“

\footnotetext{
${ }^{5}$ Nach mündlicher Auskunft des Personals vor Ort, treten diese Vorfälle häufig genug auf, um im Verlauf eines halben Jahres mögliche Veränderungen im regelwidrigen Verhalten ersehen zu können.
} 
Eine Reihe von Untersuchungen zeigten, dass die TAS eine gute innere Konsistenz (Cronbach's $\alpha=0,81$ ) und eine Retest-Reliabilität von .77 sowie eine stabile und replizierbare Faktorenstruktur aufweist (Parker, Taylor \& Bagby, 2002; Bach et al., 1996; Bagby et al.1994).

Bei der Auswertung werden fünf Items $(4,5,10,18,19)$ umgepolt, den Items werden Werte zugeordnet und es können Subskalensummen und ein Gesamtscore (von minimal 20 bis maximal 100) ermittelt werden. Der Skalenmittelwert liegt bei der männlichen Normstichprobe bei durchschnittlich 40. Werte > 60 können als auffällig gewertet werden (Bagby et al., 1994).

\subsubsection{Antisoziale Persönlichkeitsstörung}

Das Vorliegen einer Antisozialen Persönlichkeitsstörung (APS) wird mittels des Strukturierten Klinischen Interviews für DSM-IV, Achse II (Wittchen, Schramm, Zaudig \& Unland, 1993) dem SKID II (Persönlichkeitsstörungen) diagnostiziert. Aus dem Gesamtinterview wird lediglich der entsprechende diagnostische Teil der APS eingesetzt. Dieser besteht aus 19 Fragen, wobei anhand der Antworten des Probanden der Diagnostiker entscheidet, ob das dazugehörige DSM-IV-Kriterium als erfüllt angesehen werden kann.

Der erste Teil des Interviews zur Diagnostik der APS bezieht sich auf die Zeit vor dem 15. Lebensjahr ( Bsp. „Haben Sie vor Ihrem 15. Lebensjahr oft die Schule geschwänzt?“), der zweite Teil auf die Zeit danach ( Bsp. „Wie lange haben Sie in den letzten 5 Jahren nicht gearbeitet?").

Bei der Auswertung, die im Falle des SKID (-II) eine klinische Entscheidung darstellt, wird nach einem „Cutoff“ -Kriterium entschieden, das angibt, wie viele Kriterien erfüllt sein müssen, damit eine APS diagnostiziert werden kann. Im Jugendalter kann diese Diagnose lediglich als eine Tendenz zu persistierendem kriminellen bzw. sozial auffälligem Verhalten verstanden werden. Bei den Teilnehmern, die noch nicht volljährig sind, wird der Fragebogen daher nicht eingesetzt.

\subsubsection{Essaymerkmale}

Inhaltsanalytische Untersuchungen der in Schreibinterventionen produzierten Essays haben bisher verschiedene qualitative Merkmale aufgezeigt, die moderierend auf Gesundheitsvariablen wirken (vgl. 2.2.2; Pennebaker \& Francis, 1996). Um linguistische und kognitive Prozesse systematisch beobachten und auswerten zu können, wurde ein computerbasiertes Textana- 
lyseprogramm, das LIWC (Linguistic Inquiry and Word Count von Pennebaker, Francis \& Booth, 2001) entwickelt. Dieses Programm besteht aus einer Reihe von Wörterbüchern und einem Zählalgorithmus. Die Wörterbücher enthalten eine Liste von Wörtern, die grammatikalisch zusammengehören (linguistische Kategorien wie Artikel, Personalpronomen, Präpositionen) und Wörter, die bestimmte thematische Konstrukte repräsentieren (psychologische Konstrukte wie positive oder negative Emotionen, kognitive und soziale Prozesse). Das Programm geht die zuvor eingegebenen und als einzelne Dateien abgespeicherten Essays Wort für Wort durch und errechnet am Ende den Prozentsatz an Wörtern, der in jede der mehr als 70 Sprachkategorien fällt. Im Rahmen dieser Studie wird ein zur Verfügung gestelltes deutsches Wörterbuch in das Programm eingelesen und bildet analog zur englischen Version verschiedene Wörter- und Inhaltskategorien.

\section{Ergebnisse}

\subsection{Anwendbarkeit der Schreibintervention}

\subsubsection{Merkmale der Stichproben}

Im folgenden werden die Ergebnisse der Überprüfung möglicher Gruppenunterschiede hinsichtlich ausgewählter soziodemografischer und weiterer personengebundener Merkmale dargestellt.

Die Ergebnisse der varianzanalytischen Überprüfung* zeigen keine signifikanten Gruppenunterschiede zum Zeitpunkt der Ersterhebung hinsichtlich:

- des Alters

- des Alters bei erstmaliger Straffälligkeit

- der Straflänge

- des Zeitraums der bisherigen Inhaftierung

- des Ausmaßes an Alexithymie

- der aktuellen Stimmung

- der subjektiven Belastung durch die Inhaftierung

\footnotetext{
* Alle im folgenden berichteten statistischen Analysen wurden unter Verwendung des Statistikprogramms SPSS, Version 12.0 durchgeführt.
} 
Tabelle 5

Mittelwerte, Standardabweichungen und Ergebnisse der einfaktoriellen Varianzanalyse verschiedener Stichprobenmerkmale $(\mathrm{N}=60 ; \mathrm{df}=2)$

\begin{tabular}{l|l|c|c|c|c|c}
\hline Variable & Gruppe & $\mathbf{n}$ & $\mathbf{M}$ & $\mathbf{S D}$ & $\mathbf{F}$ & $\mathbf{p}$ \\
\hline Alter & KG & 20 & 20,08 & 1,78 & & \\
& BE & 20 & 19,45 & 1,47 & 0,685 & n.s. \\
& TA & 20 & 19,65 & 1,90 & & \\
\hline Alter bei & KG & 20 & 13,90 & 3,71 & & \\
erster Straffälligkeit & BE & 20 & 13,05 & 3,30 & 0,295 & n.s. \\
& TA & 20 & 13,45 & 3,49 & & \\
\hline Straflänge & KG & 20 & 16,00 & 7,53 & & \\
in Monaten & BE & 20 & 14,05 & 7,72 & 0,314 & n.s. \\
& TA & 20 & 15,10 & 8,12 & & \\
\hline Zeitraum bisheriger & KG & 20 & 36,20 & 21,57 & & \\
Inhaftierung (in Mona- & BE & 20 & 37,55 & 32,07 & 0,118 & n.s. \\
ten) & TA & 20 & 40,35 & 27,90 & & \\
\hline Alexithymie & KG & 20 & 52,65 & 12,19 & & \\
TAS-20 & BE & 20 & 52,10 & 14,38 & 0,189 & n.s. \\
& TA & 20 & 54,50 & 12,10 & & \\
\hline Aktuelle Stimmung & KG & 20 & 3,05 & 1,05 & & \\
(von 1= sehr gut bis & BE & 20 & 3,00 & 0,65 & 0,149 & n.s. \\
5= sehr schlecht) & TA & 20 & 2,90 & 0,91 & & \\
\hline Belastung durch & KG & 20 & 2,65 & 0,87 & & \\
Haft (von 1= gar nicht & BE & 20 & 3,00 & 0,92 & 2,389 & n.s. \\
bis 4= sehr) & TA & 20 & 2,40 & 0,82 & & \\
\hline & & & & & & \\
& & & & & & \\
\hline
\end{tabular}

Ein Häufigkeitsvergleich der verschiedenen Schulabschlüsse der Teilnehmer in den drei Gruppen ergibt keine signifikanten Gruppenunterschiede $\left(\mathrm{Chi}^{2}(\mathrm{df}=8)=5,328 ; \mathrm{p}=0,772\right)$.

Betrachtet man die Häufigkeitsverteilung des Vorliegens einer Antisozialen Persönlichkeitsstörung (APS) in den Gruppen, so zeigt sich ein heterogenes Bild. Bei den 56 volljährigen Teilnehmern erfüllen 19 die Kriterien einer APS. Trotz randomisierter Zuweisung der Probanden zu den drei Gruppen, befinden sich 9 der Betroffenen in der Gruppe TA und lediglich 4 Teilnehmer in der Kontroll- und 6 in der Gruppe BE. Die teststatistische Überprüfung mittels $\mathrm{Chi}^{2}$-Test (Kreuztabelle) ergibt allerdings keine signifikanten Gruppenunterschiede $\left(\mathrm{Chi}^{2}(\mathrm{df}=2)=3,526 ; \mathrm{p}=0,172\right)$.

Von chronischen Krankheiten betroffen zu sein, geben 10 aller Teilnehmer an. Auch hier befindet sich die Hälfte der Personen in der Gruppe TA und lediglich ein Teilnehmer in der $\mathrm{KG}$, es ergeben sich statistisch erneut keine signifikanten Gruppenunterschiede $\left(\mathrm{Chi}^{2}(\mathrm{df}=2)=\right.$ $3,120 ; p=0,210)$. 


\subsubsection{Kurzfristige affektive Reaktionen}

Ein erhöhter Wert aktuellen negativen Affekts direkt nach der Schreibsitzung wird als Hinweis auf eine emotionale Involvierung der Person während der Schreibsitzung und damit als ein erfolgreicher Manipulationcheck gewertet. Angenommen wurde, dass sich bei den Teilnehmern der Experimentalgruppen (BE und TA) eine kurzfristige Stimmungsverschlechterung zeigt.

Die über alle drei Schreibtage gemittelte Werte positiven Affekts zeigen eine signifikante Stimmungsverschlechterung für die Teilnehmer von TA $(\mathrm{t}=2,180 ; \mathrm{p}(2$-seitig $)=0,041)$ und BE $(\mathrm{t}=2,616 ; \mathrm{p}(2$-seitig $)=0,016)$ nach dem Schreiben (siehe Abbildung 5). Bezüglich des negativen Affekts zeigt sich eine signifikante Stimmungsverschlechterung in der Gruppe TA $(\mathrm{t}=$ 4,761; $\mathrm{p}(2$-seitig $)=0,000)$ und eine kurzfristige signifikante Stimmungsverbesserung in der Kontrollgruppe $(\mathrm{t}=3,187 ; \mathrm{p}(2$-seitig $)=0,005)$.
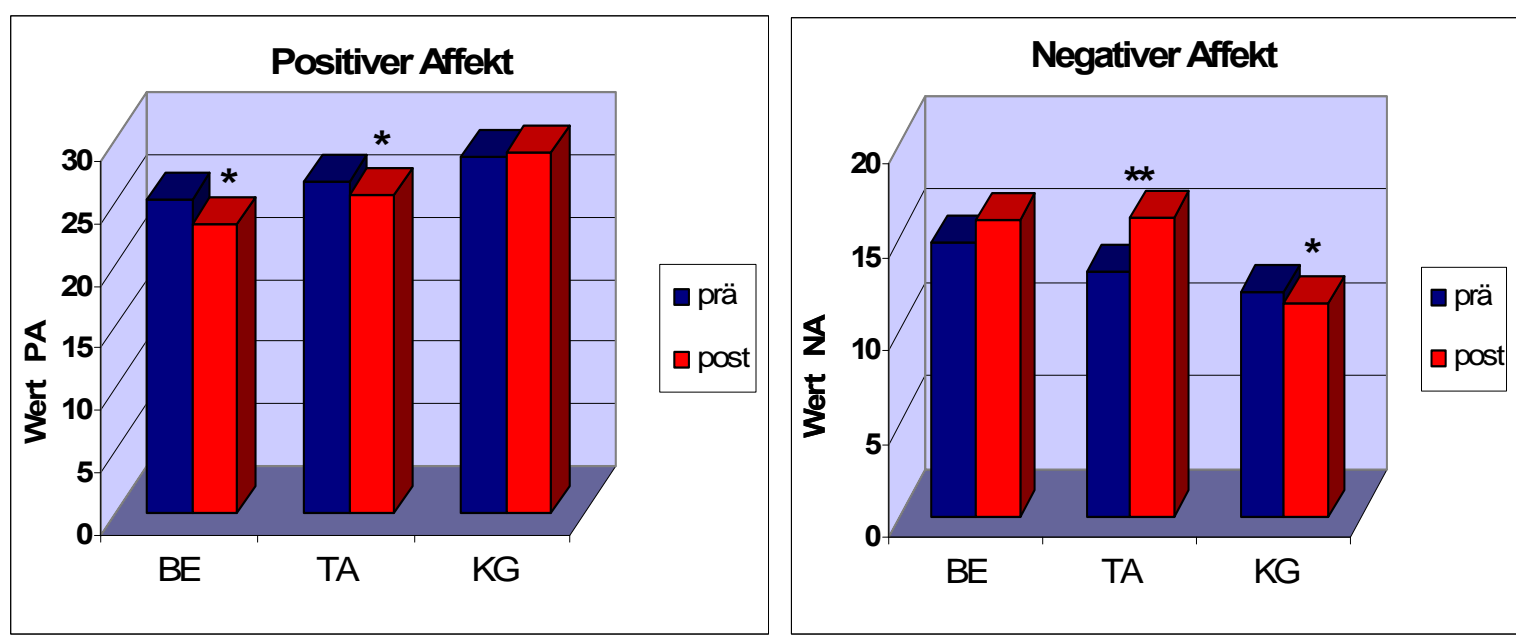

Abbildung 5: Intragruppenvergleiche kurzfristigen Positiven und Negativen Affekts (PA NAS), über alle Schreibtage gemittelte Werte; ${ }^{*} \mathrm{p}<.05 ;{ }^{* *} \mathrm{p}<.01$.

Eine statistische Überprüfung möglicher Gruppenunterschiede mittels zweifaktorieller Varianzanalyse mit Messwiederholung liefert hinsichtlich beider Variablen Negativer Affekt (NA) und Positiver Affekt (PA) einen erwartungsgemäß hochsignifikanten Interaktionseffekt der Faktoren Bedingung und Zeitpunkt (vgl. Tabelle 6). Post-hoc Testungen ergeben bezüglich NA signifikante Unterschiede zwischen den Gruppen BE und KG $(F(1,40)=6,25$; $\mathrm{p}=0,017)$ sowie den Gruppen TA und $\mathrm{KG}(\mathrm{F}(1,39)=28,99 ; \mathrm{p}<0,000)$. Das Ausmaß schlechter Stimmung nimmt in den Experimentalgruppen hypothesenkonform zu und in der Kontrollgruppe ab. Bezüglich PA zeigt sich ein spiegelverkehrtes Bild mit relevanten Unterschieden zwischen den Gruppen BE und $\mathrm{KG}(\mathrm{F}(1,39)=6,19 ; \mathrm{p}=0,017)$ und den Gruppen TA und KG 
$(\mathrm{F}(1,39)=4,25 ; \mathrm{p}=0,046)$, wobei das Ausmaß guter Stimmung in den Experimentalgruppen abnimmt und in der Kontrollgruppe zunimmt.

Tabelle 6

Intergruppenvergleiche kurzfristigen Affekts: Mittelwerte, Standardabweichungen, Ergebnisse der zweifaktoriellen Varianzanalyse mit Messwiederholung

\begin{tabular}{|c|c|c|c|c|c|c|c|c|}
\hline \multirow{4}{*}{$\begin{array}{l}\text { Negativer } \\
\text { Affekt }\end{array}$} & \multirow{2}{*}{ Gruppe } & \multicolumn{3}{|c|}{ Prä } & \multicolumn{2}{|c|}{ Post } & \multicolumn{2}{|c|}{$\begin{array}{c}\text { Zeit x Bedingung } \\
(\mathrm{df}=2,61)\end{array}$} \\
\hline & & $n$ & $\bar{M}$ & $\overline{\text { SD }}$ & $\bar{M}$ & $\overline{S D}$ & $F$ & p \\
\hline & KG & 20 & 12,08 & 1,82 & 11,48 & 1,69 & \multirow{3}{*}{10,079} & \multirow{3}{*}{$0,000^{* *}$} \\
\hline & $\mathrm{BE}$ & 22 & 14,68 & 4,02 & 15,68 & 5,24 & & \\
\hline & TA & 21 & 13,16 & 3,89 & 16,02 & 5,04 & & \\
\hline \multirow{3}{*}{$\begin{array}{l}\text { Positiver } \\
\text { Affekt }\end{array}$} & KG & 20 & 28,73 & 6,93 & 29,02 & 6,80 & \multirow{3}{*}{3,454} & \multirow{3}{*}{$0,038^{*}$} \\
\hline & BE & 22 & 25,22 & 7,88 & 23,32 & 8,45 & & \\
\hline & TA & 21 & 26,79 & 6,48 & 25,60 & 7,12 & & \\
\hline
\end{tabular}

$* \mathrm{p}<.05,{ }^{* *} \mathrm{p}<.01$.

Eine Überprüfung der Ausgangswerte ergibt jedoch für NA einen signifikanten Unterschied zwischen den Gruppen BE und KG ( $\mathrm{t}=-2,651$; $\mathrm{p}(2$-seitig $)=0,01)$ mit einem höheren Mittelwert der Gruppe BE. Ein möglicher systematischer Einfluss der unterschiedlichen Ausgangswerte als Kovariate zeigt sich allerdings im Rahmen einer statistischen Überprüfung mittels Kovarianzanalyse als nicht signifikant $(F(2,63)=0,286 ; p=0,595)$.

\subsubsection{Inhalte der Essays}

Alle 60 Teilnehmer fertigten jeweils drei Essays an. Eine inhaltliche Durchsicht ergibt, dass die Teilnehmer der KG ohne Ausnahme über ihren Tagesablauf während der Inhaftierung berichteten, belastende Lebensereignisse oder Ereignisse, die mit der eigenen Straffälligkeit in Verbindung stehen, wurde nicht berichtet. Eine kategoriale Zusammenfassung der Themen, über die in den beiden Experimentalgruppen geschrieben wurde, zeigt, dass auch diese Gruppen sich inhaltlich an die Schreibinstruktion gehalten haben und ausschließlich über belastende Lebensereignisse, hauptsächlich über Krankheit oder Tod eines Angehörigen oder nahe stehenden Menschen bzw. ihre Straftaten geschrieben haben. 
Tabelle 7

Kategorisierung der Essayinhalte

\begin{tabular}{|l|c|}
\hline Gruppe: Belastendes Lebensereignis (n=19) & Häufigkeit \\
\hline $\begin{array}{l}\text { Tod (oder bedrohliche Erkrankung) eines Angehörigen oder nahe } \\
\text { stehenden Menschen }\end{array}$ & $9(47 \%)$ \\
Trennung oder Konflikte der Eltern & $3(16 \%)$ \\
Konflikte mit Familienmitgliedern & $3(16 \%)$ \\
Heimunterbringung & $3(16 \%)$ \\
anderes & $1(5 \%)$ \\
& $10(50 \%)$ \\
\hline Gruppe: Tataufarbeitung (n=20) & $6(30 \%)$ \\
Diebstahl/Raub und Einbruchsdelikte & $4(20 \%)$ \\
\hline Körperverletzungsdelikte & \\
Betrugs- und Drogendelikte & \\
\hline
\end{tabular}

Anmerkung: Ein Essay der Gruppe BE war unleserlich.

Die in den Tabellen 8 bis 10 wiedergegebene Auszüge dreier Essays verdeutlichen beispielhaft sowohl ihren Inhalt wie ihre Form.

Tabelle 8

Beispielessay von P. (17 Jahre, Gruppe BE)

In meinem Leben gibt es sehr viele schlimme und belastende Ereignisse, sei es nun, dass ich so ziemlich ohne Vater aufgewachsen bin, dass er meine Mutter vergewaltigt, verprügelt und beinahe umgebracht hat, dass ich über zehnmal umgezogen bin, dass meine Mutter mich nicht bei sich haben wollte, dass ich in der Kinder- und Jugendpsychiatrie war, dass ich mehrmals nur knapp dem Tod entronnen bin oder dass ich von meinem Pflegevater vergewaltigt worden bin. Das eindeutig schlimmste Ereignis meines Lebens bezog sich auf ein Mädchen, meine größte Schwäche. Vor zweieinhalb Jahren lernte ich V., so hieß sie, im „Kinderhaus zur M.“, das Heim, in dem ich untergebracht war, kennen. Ich verliebte mich sofort in die und wusste, dass sie der einzige Mensch ist, mit der ich hätte glücklich werden können... Sie kam aus derselben, 200 km weit entfernten Psychiatrie wie ich, wo sie wegen besonders schwerem Suizid war. Wir haben zwar nie viel geredet, aber ich bekam schnell heraus, das sie von ihrem Vater vergewaltigt wurde, Das band uns noch enger aneinander... 
Tabelle 9:

Beispielessay von R. (21 Jahre, Gruppe TA)

Ich war in der Schule, als ein Russe zu mir kam und mich angespuckt hat. Es war mir peinlich und ich wollte ausrasten, doch ein Lehrer kam um die Ecke. Am Abend war eine Schulfete und ich war mit Kumpels da. Ich gehörte damals der rechten Szene an. Der Russe war auch da und ich habe inn in der Halle, die als Disko umfunktioniert war, gesehen. Ich hasste inn, seine arrogante Art und seinen Akzent, er kotzte mich an. Ich bin raus gegangen und habe einen Kumpel von mir erzählt was los war und dass er da ist. Wir haben auf inn gewartet. Als er mit seiner Freundin raus kam und nach Hause gehen wollte, sind ich, ein Freund und meine Freundin inn mit einem Corsa bis zu einem Feldweg gefolgt und haben ihn dann mit dem Auto den Weg abgeschnitten. Meine damalige Freundin hat sich um seine Alte gekümmert und ich habe mir den dreckigen Russen geschnappt. Ich hatte Angst und Spaß zugleich. Wir haben inn solange zu Brei gehauen bis ein anderes Auto kam und sie mitgenommen hat...

Tabelle 10

Beispielessay von S. (24 Jahre, Gruppe KG)

Gestern morgen hab ich erstmal gefrühstückt. Müsli und Schokobrot! Dann hab ich ein wenig meine Zelle sauber gemacht. Dann bin ich zu meiner Arbeitsstelle gegangen und habe ich ein Werkstück bearbeitet. Einen so genannten „Teufelsknoten“ aus Holz. Ich habe sehr präzise gearbeitet und sehr ordentlich. Wurde sehr gut benotet. Nach der Arbeit habe ich einen Brief an meine Freundin geschrieben und habe ein bisschen trainiert. Den Oberkörper sowie Arme. Nachdem ich damit fertig war, habe ich mich geduscht und noch ein wenig ferngesehen. Die Comet-Verleihung und danach lief eine englische Komödie. Habe auch 1-2 Tassen Tee getrunken und noch mit meiner Freundin telefoniert. Tja, dann bin ich auch schon zu Bett gegangen. Ich habe keine Drogen genommen, obwohl es mir angeboten wurde. Ich schlief sehr schnell ein. Der Nachtdienst kam zweimal vorbei, so dass ich wach wurde. Bin aber immer wieder gleich eingeschlafen.

\subsubsection{Bewertung des Schreibens}

Mittels postexperimentellen Fragebogen (Anhang B15) gaben die Teilnehmer direkt nach jeder Schreibsitzung ihre Gefühle in Bezug auf das Schreiben und der möglichen wahrgenommenen Belastung an, die damit einherging. 
Tabelle 11

Bewertung der Schreibgruppen: Mittelwerte, Standardabweichungen (gemittelt über drei Schreibtage), Ergebnisse der t-Tests mit unabhängigen Stichproben; $\mathrm{df}=38$

Wie belastend ist das, was Sie heute geschrieben haben?

Wie persönlich ist das, was Sie heute geschrieben haben?

Hat Sie das Schreiben

heute belastet?

Hat Sie das Schreiben heute erleichtert?

Wie stark haben Sie Ihre

Gefühle ausgedrückt?

Fortsetzung nächste Seite

Ist es Ihnen schwer gefallen, beim Schreiben Ihre Gefühle auszudrücken?

Wie oft haben Sie mit anderen bisher über das Thema gesprochen?

Haben Sie es bisher bewusst vermieden, über das Thema zu sprechen?

\begin{tabular}{|c|c|c|c|c|c|}
\hline \multirow{2}{*}{\multicolumn{2}{|c|}{ M }} & \multirow[b]{2}{*}{ SD } & \multicolumn{3}{|c|}{ Gruppenvergleiche } \\
\hline & & & $\begin{array}{c}\text { BE-KG } \\
t^{a} / p\end{array}$ & $\begin{array}{l}\text { TA-KG } \\
t^{a} / p\end{array}$ & $\begin{array}{l}\text { BE-TA } \\
t^{a} / p\end{array}$ \\
\hline $\begin{array}{l}\text { BE } \\
\text { TA } \\
\text { KG }\end{array}$ & $\begin{array}{l}3,32 \\
3,43 \\
1,30\end{array}$ & $\begin{array}{l}0,69 \\
1,18 \\
0,69\end{array}$ & $\begin{array}{c}-5,239 * * * l \\
0,000\end{array}$ & $\begin{array}{c}-6,980 * * * \\
10,000\end{array}$ & n.s. \\
\hline $\begin{array}{l}\text { BE } \\
\text { TA } \\
\text { KG }\end{array}$ & $\begin{array}{l}5,35 \\
4,28 \\
2,22\end{array}$ & $\begin{array}{l}1,25 \\
1,27 \\
1,03\end{array}$ & $\begin{array}{c}-8,643 * * * 1 \\
0,000\end{array}$ & $\begin{array}{c}-5,624 * * * \\
10,000\end{array}$ & $\begin{array}{c}2,689 * I \\
0,011\end{array}$ \\
\hline $\begin{array}{l}\text { BE } \\
\text { TA } \\
\text { KG }\end{array}$ & $\begin{array}{l}2,93 \\
2,63 \\
1,32\end{array}$ & $\begin{array}{l}1,63 \\
1,18 \\
0,73\end{array}$ & $\begin{array}{c}-4,046 * * * l \\
0,000\end{array}$ & $\begin{array}{c}-4,213 * * * I \\
0,000\end{array}$ & n.s. \\
\hline $\begin{array}{l}\text { BE } \\
\text { TA } \\
\text { KG }\end{array}$ & $\begin{array}{l}2,50 \\
3,36 \\
2,07\end{array}$ & $\begin{array}{l}1,37 \\
1,46 \\
1,07\end{array}$ & n.s. & $\begin{array}{c}-3,188 * * I \\
0,003\end{array}$ & n.s. \\
\hline $\begin{array}{l}\text { BE } \\
\text { TA } \\
\text { KG }\end{array}$ & $\begin{array}{l}4,15 \\
4,01 \\
1,32\end{array}$ & $\begin{array}{l}1,54 \\
1,18 \\
0,38\end{array}$ & $\begin{array}{c}-7,973 * * * l \\
0,000\end{array}$ & $\begin{array}{c}-9,757 * * * l \\
0,000\end{array}$ & n.s. \\
\hline $\begin{array}{l}\text { BE } \\
\text { TA } \\
\text { KG }\end{array}$ & $\begin{array}{l}4,05 \\
3,73 \\
1,50\end{array}$ & $\begin{array}{l}1,93 \\
1,30 \\
1,23\end{array}$ & $\begin{array}{c}-4,989 * * * l \\
0,000\end{array}$ & $\begin{array}{c}-5,565 * * * \mid \\
0,000\end{array}$ & n.s. \\
\hline $\begin{array}{l}\text { BE } \\
\text { TA } \\
\text { KG }\end{array}$ & $\begin{array}{l}2,90 \\
2,93 \\
1,73\end{array}$ & $\begin{array}{l}1,21 \\
1,44 \\
0,81\end{array}$ & $\begin{array}{c}-3,598 * * l \\
0,001\end{array}$ & $\begin{array}{c}-3,267 * * l \\
0,002\end{array}$ & n.s. \\
\hline $\begin{array}{l}\text { BE } \\
\text { TA } \\
\text { KG }\end{array}$ & $\begin{array}{l}4,23 \\
3,58 \\
2,38\end{array}$ & $\begin{array}{l}1,72 \\
1,81 \\
1,88\end{array}$ & $\begin{array}{c}-3,246 * * I \\
0,002\end{array}$ & $\begin{array}{c}-2,046 * l \\
0,048\end{array}$ & n.s. \\
\hline
\end{tabular}

Anmerkungen: Die Antwortskala reicht von 1=,gar nicht“ bis 7=,,sehr stark“.

${ }^{a}$ Alle $\mathrm{t}-$ Werte sind zweiseitig

$* \mathrm{p}<.05, * * \mathrm{p}<.01, * * * \mathrm{p}<.001$.

Im Gruppenvergleich (t-Test bei unabhängigen Stichproben) zeigt sich, dass die Teilnehmer der beiden Experimentalgruppen das Schreiben erwartungsgemäß als belastender empfunden haben. Außerdem haben sie den Inhalt ihrer Essays als persönlicher wahrgenommen, haben dabei stärker ihre Gefühle ausgedrückt, wobei ihnen dieses aber auch schwerer gefallen ist. Bisher haben sie seltener über das Thema gesprochen und dies auch bewusst vermieden als die Teilnehmer der Kontrollgruppe. Lediglich die Teilnehmer der Gruppe TA, die über ihre vergangenen Straftaten geschrieben haben, haben sich durch das Schreiben signifikant erleichterter gefühlt als die Kontrollgruppe. 


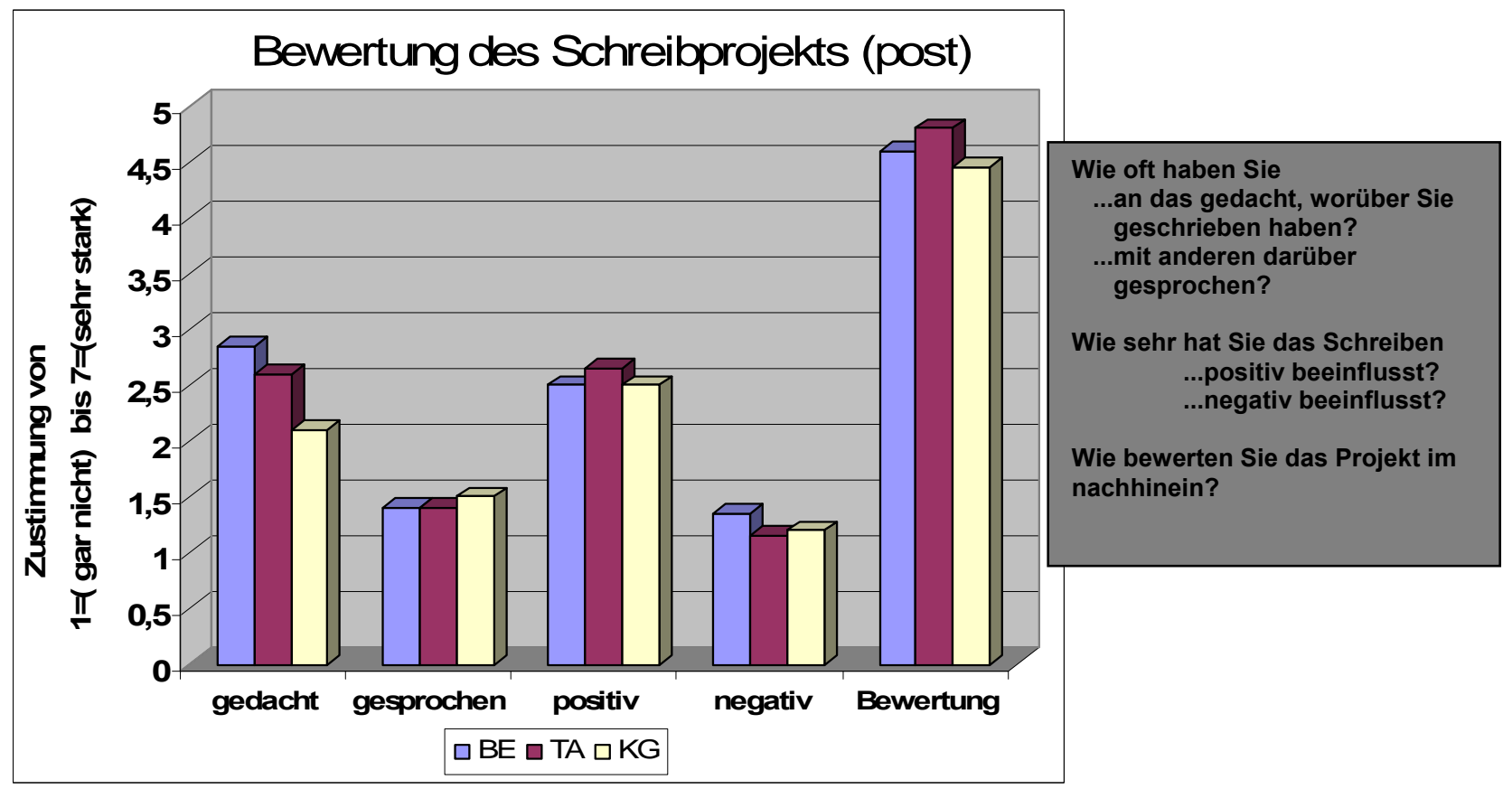

Abbildung 6: Durchschnittliche Bewertung des Projektes der drei Gruppen

Wie aus Abbildung 6 hervorgeht, haben alle Teilnehmer das Projekt im nachhinein (acht Wochen danach) grundsätzlich positiv bewertet. Eine (persönliche) positive Beeinflussung durch das Projekt schätzen die Teilnehmer für sich selbst eher mittelmäßig ein.

Sie geben an, in der Folge häufiger an das Schreibthema gedacht als darüber mit anderen gesprochen zu haben. Eine varianzanalytische Überprüfung hinsichtlich möglicher Gruppenunterschiede in der Bewertung des Projektes hat keinerlei signifikanter Unterschiede ergeben. Ausformulierte Kommentare der Teilnehmer zu Studie befinden sich in Anhang C.

\subsection{Langfristige Effekte Expressiven Schreibens}

Mögliche langfristige Effekte des ES auf gesundheitsbezogene Maße, Maße der Emotionsregulation sowie der Normorientierung und der Führung wurden jeweils durch mehrere Variablen erhoben. Die Hypothesenprüfung erfolgt mittels zweifaktorieller multivariater Varianzanalyse mit Messwiederholung und einem zweifachgestuften Innersubjektfaktor (Zeit) sowie einem dreifachgestuften Zwischensubjektfaktor (Gruppe). Entscheidend für die Hypothesenprüfung sind die mittels Varianzanalyse berechneten Interaktionseffekte, eventuelle Haupteffekte werden lediglich der Vollständigkeit halber dargestellt. 


\subsubsection{Veränderungen in Gesundheitsvariablen}

Um eine mögliche längerfristige Wirksamkeit von ES auf Maße körperlicher und psychischer Gesundheit zu überprüfen, wurde zunächst mittels einfaktorieller Varianzanalyse überprüft, ob zwischen den Ausgangswerten der verschiedenen Variablen Unterschiede bestehen. Es zeigen sich keine signifikanten Gruppenunterschiede (vgl. Tabelle 12)

Tabelle 12

Ergebnisse der Überprüfung der Ausgangswerte (prä): Mittelwerte, Standardabweichungen und Ergebnisse der einfaktoriellen Varianzanalyse

\begin{tabular}{|c|c|c|c|c|c|}
\hline & \multicolumn{2}{|c|}{ Prä } & \multicolumn{2}{|c|}{$\begin{array}{l}\text { ANOVA } \\
(\mathrm{df}=2,56)\end{array}$} \\
\hline & & $\mathbf{M}$ & SD & $F$ & $p$ \\
\hline $\begin{array}{l}\text { Anzahl } \\
\text { Krankheitstage }\end{array}$ & $\begin{array}{l}\text { BE } \\
\text { TA } \\
\text { KG }\end{array}$ & $\begin{array}{l}1,50 \\
1,40 \\
1,10\end{array}$ & $\begin{array}{l}2,65 \\
3,17 \\
1,92\end{array}$ & 0,703 & n.s. \\
\hline $\begin{array}{l}\text { Anzahl } \\
\text { Arztbesuche }\end{array}$ & $\begin{array}{l}\text { BE } \\
\text { TA } \\
\text { KG }\end{array}$ & $\begin{array}{l}1,70 \\
1,68 \\
0,85\end{array}$ & $\begin{array}{l}1,26 \\
2,41 \\
0,67\end{array}$ & 1,834 & n.s. \\
\hline $\begin{array}{l}\text { Körperliche } \\
\text { Beschwerden } \\
\text { (Beschwerdeliste) }\end{array}$ & $\begin{array}{c}\text { BE } \\
\text { TA } \\
\text { KG }\end{array}$ & $\begin{array}{l}17,70 \\
15,89 \\
11,55\end{array}$ & $\begin{array}{c}11,16 \\
10,89 \\
8,33\end{array}$ & 1,921 & n.s. \\
\hline $\begin{array}{l}\text { Psychische } \\
\text { Symptombelastung } \\
\text { (SCL-K9) }\end{array}$ & $\begin{array}{l}\text { BE } \\
\text { TA } \\
\text { KG }\end{array}$ & $\begin{array}{l}1,07 \\
1,25 \\
0,99\end{array}$ & $\begin{array}{l}0,66 \\
0,89 \\
0,78\end{array}$ & 0,554 & n.s. \\
\hline
\end{tabular}

Die Ergebnisse der teststatistischen Überprüfung möglicher Gruppenunterschiede im präpost-Vergleich für die abhängigen Variablen Anzahl der Krankheitstage und Anzahl der Arztbesuche (der letzten 6 Wochen), sowie das Ausmaß körperlicher Beschwerden und der psychischen Symptombelastung sind in Tabelle 13 dargestellt. 
Tabelle 13

Gesundheitsvariablen: Mittelwerte, Standardabweichungen und Ergebnisse der zweifaktoriellen Varianzanalyse mit Messwiederholung

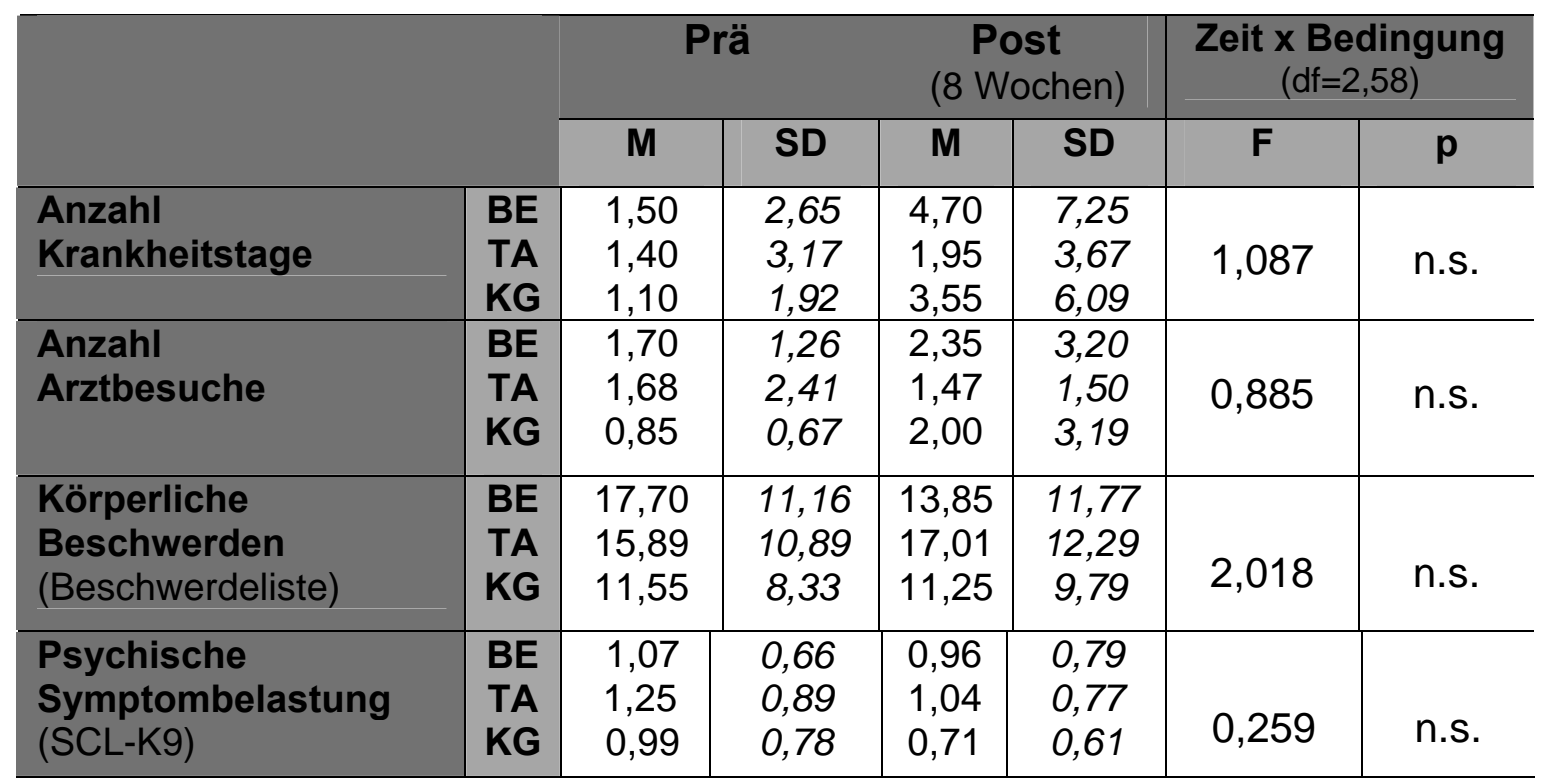

Es zeigen sich keinerlei Interaktionseffekte hinsichtlich der erhobenen Gesundheitsmaße. Demnach kommt es - entgegen der Hypothese - in den Experimentalgruppen zu keinerlei langfristiger Verbesserung der Gesundheit im Vergleich zur Kontrollgruppe. Lediglich der Haupteffekt Zeit ergibt eine signifikante Abnahme der psychischen Belastung ((SCL-K) $\mathrm{F}(1,59)=4,758 ; \mathrm{p}=0,033)$, sowie eine signifikante Zunahme der Anzahl der Krankheitstage ( $\mathrm{F}$ $(1,59)=7,464 ; p=0,008)$ über alle Bedingungen, wobei sich auffallend hohe Standardabweichungen zum Zeitpunkt der post-Erhebung ergeben.

Bezüglich der Angaben zur Häufigkeit von Alkohol- und Drogenkonsum der vergangenen 6 Wochen ist lediglich eine eingeschränkte Auswertung möglich. Ein prä-post-Vergleich ist aufgrund der nahe bei Null liegenden prä-Werte nicht möglich, zudem sind beide inhaltlich wenig vergleichbar, da sich der Drogenkonsum zum ersten Erhebungszeitpunkt bei vielen Teilnehmern noch auf die Zeit vor der Inhaftierung bezieht (und damit auch ein anderer Zugang zu Alkohol und Drogen bestand). Dies trifft allerdings nicht auf alle Teilnehmer in gleichem Maß zu, da sie zum Zeitpunkt der Erstuntersuchung bereits unterschiedlich lang inhaftiert waren. Daher werden lediglich die post-Werte der Gruppen varianzanalytisch verglichen. Es zeigen sich keine Gruppenunterschiede bezüglich Alkohol- $(\mathrm{F}(2,57)=0,036$; n.s. $)$ und Drogenkonsum $(\mathrm{F}(2,57)=1,338 ;$ n.s. $)$.

Das Ausmaß der durch das im Essay beschriebene Erlebnis hervorgerufene Belastungsreaktionen ist in den beiden Experimentalgruppen (BE und TA) unmittelbar nach der ersten und acht Wochen nach der letzten Schreibsitzung erhoben worden. Der Vergleich der prä-Werte 
zeigt keine signifikanten Gruppenunterschiede (siehe Tabelle 14). Es ergeben sich keinen signifikanten Gruppenunterschiede bezüglich der drei Skalen Intrusion, Hyperarousal und Vermeidung. Für beide Gruppen ergibt sich aber hypothesenkonform eine hochsignifikante Abnahme des Ausmaßes der drei Belastungsreaktionen über die Zeit.

Tabelle 14

Belastungsreaktionen: Mittelwerte, Standardabweichungen und Ergebnisse der einfaktoriellen Varianzanalyse über die prä-Werte sowie der zweifaktoriellen Varianzanalyse (Zeit x Bedingung) mit Messwiederholung $(\mathrm{n}=40)$

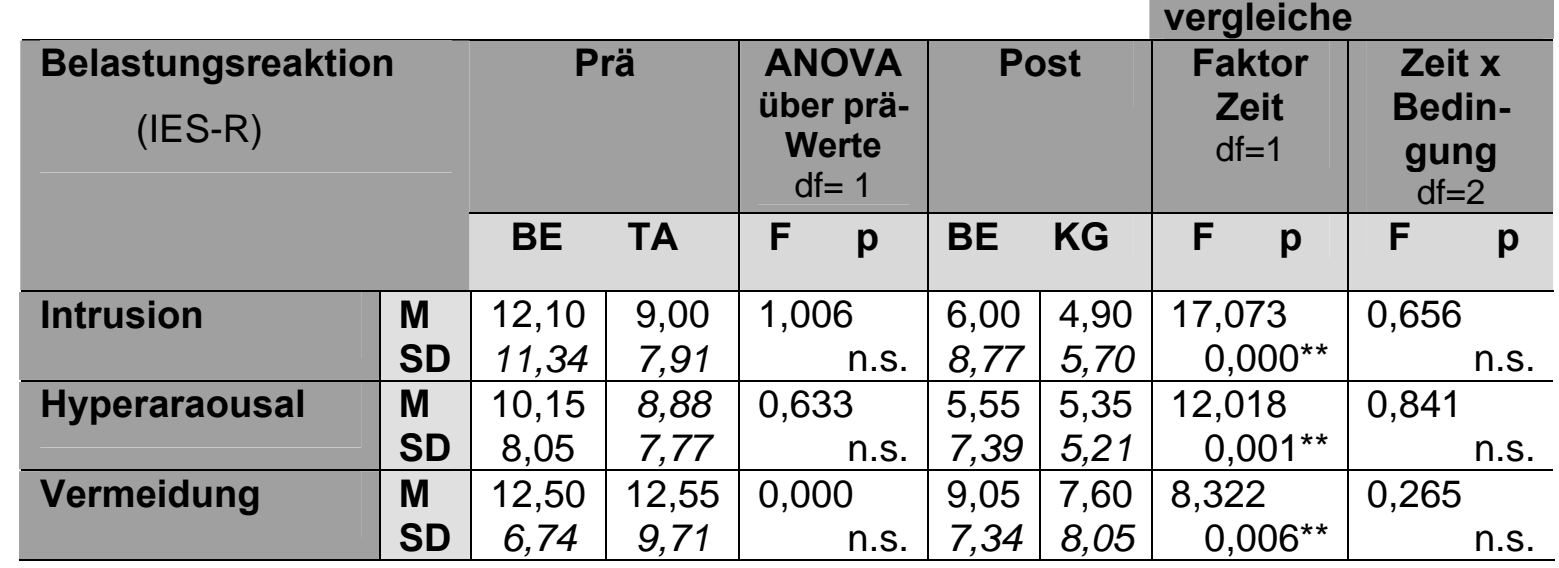

$*<.05,{ }^{* *} \mathrm{p}<.01$.

\subsubsection{Veränderungen in Emotionsvariablen}

Die Maße von Depressivität sowie Zustandsangst und -ärger zeigen zum Zeitpunkt der Ersterhebung in ihrer Ausprägung keinerlei Gruppenunterschiede. Lediglich in der Subskala Positiver Affekt (PA) zeigt sich varianzanalytisch ein signifikanter Gruppenunterschied (vgl. Tabelle 15). Post-hoc t-Tests ergeben, dass sich die Gruppen TA und KG in ihren Ausgangswerten unterscheiden $(\mathrm{t}=-2,862 ; \mathrm{df}=37 ; \mathrm{p}(2$-seitig $)=0,007)$, wobei die Kontrollgruppe eine signifikant höhere Ausprägung an PA aufweist $(M=31,18 ; \mathrm{SD}=5,03)$ als die Gruppe TA $(M=26,40$; $\mathrm{SD}=6,78)$. Zum Zeitpunkt der Folgeuntersuchung unterscheiden sich die beiden Gruppen hinsichtlich des PA nicht mehr (KG zeigt eine Abnahme, TA eine Zunahme an PA). 
Tabelle 15

Emotionsbezogene Maße: Mittelwerte und Standardabweichungen (prä und post), Ergebnisse der einfaktoriellen Varianzanalyse (prä-Werte)

\begin{tabular}{|c|c|c|c|c|c|c|c|c|}
\hline \multirow{2}{*}{\multicolumn{2}{|c|}{ Variablen }} & \multirow[b]{2}{*}{$\mathbf{n}$} & \multicolumn{2}{|c|}{ Prä } & \multicolumn{2}{|c|}{$\begin{array}{c}\text { Post } \\
\text { (8 Wochen) }\end{array}$} & \multicolumn{2}{|c|}{$\begin{array}{c}\text { ANOVA } \\
\text { (prä-Werte) } \\
\text { df=2 }\end{array}$} \\
\hline & & & $\mathbf{M}$ & SD & SD & $\mathbf{M}$ & $\mathbf{F}$ & $\mathbf{p}$ \\
\hline $\begin{array}{l}\text { Depressivität } \\
\text { (ADS) }\end{array}$ & $\begin{array}{l}\text { BE } \\
\text { TA } \\
\text { KG } \\
\end{array}$ & $\begin{array}{l}19 \\
20 \\
18 \\
\end{array}$ & $\begin{array}{l}15,84 \\
16,89 \\
16,00 \\
\end{array}$ & $\begin{array}{l}7,58 \\
8,92 \\
9,50 \\
\end{array}$ & $\begin{array}{l}12,63 \\
13,50 \\
10,12 \\
\end{array}$ & $\begin{array}{c}9,52 \\
10,89 \\
8,22 \\
\end{array}$ & 0,130 & n.s. \\
\hline $\begin{array}{l}\text { Habituelle } \\
\text { Affektivität } \\
\text { (PANAS) } \\
\text { Positiver Affekt }\end{array}$ & $\begin{array}{l}\text { BE } \\
\text { TA } \\
\text { KG }\end{array}$ & $\begin{array}{l}19 \\
20 \\
18\end{array}$ & $\begin{array}{l}28,42 \\
29,94 \\
32,18\end{array}$ & $\begin{array}{l}6,35 \\
7,60 \\
5,02\end{array}$ & $\begin{array}{l}27,95 \\
27,50 \\
29,82\end{array}$ & $\begin{array}{l}936 \\
6,62 \\
6,41\end{array}$ & 3,449 & $0,039 *$ \\
\hline Negativer Affekt & $\begin{array}{l}\text { BE } \\
\text { TA } \\
\text { KG }\end{array}$ & $\begin{array}{l}19 \\
20 \\
18\end{array}$ & $\begin{array}{l}23,16 \\
21,56 \\
20,88\end{array}$ & $\begin{array}{l}6,13 \\
7,23 \\
7,57\end{array}$ & $\begin{array}{l}18,63 \\
18,50 \\
16,82\end{array}$ & $\begin{array}{l}6,84 \\
7,28 \\
7,60\end{array}$ & 0,467 & n.s. \\
\hline $\begin{array}{l}\text { Zustandsangst } \\
\text { (STAI) }\end{array}$ & $\begin{array}{l}\text { BE } \\
\text { TA } \\
\text { KG }\end{array}$ & $\begin{array}{l}19 \\
20 \\
18\end{array}$ & $\begin{array}{l}45,00 \\
40,00 \\
42,35\end{array}$ & $\begin{array}{c}10,92 \\
7,71 \\
10,49\end{array}$ & $\begin{array}{l}40,42 \\
41,22 \\
39,94\end{array}$ & $\begin{array}{l}8,54 \\
8,41 \\
6,63\end{array}$ & 0,994 & n.s. \\
\hline $\begin{array}{l}\text { Zustandsärger } \\
(S T A X I)\end{array}$ & $\begin{array}{l}\text { BE } \\
\text { TA } \\
\text { KG }\end{array}$ & $\begin{array}{l}19 \\
20 \\
18\end{array}$ & $\begin{array}{l}14,63 \\
11,33 \\
12,18\end{array}$ & $\begin{array}{l}5,49 \\
3,07 \\
3,76\end{array}$ & $\begin{array}{l}12,00 \\
14,50 \\
13,12\end{array}$ & $\begin{array}{l}4,09 \\
6,59 \\
6,12\end{array}$ & 0,920 & n.s. \\
\hline
\end{tabular}

$*_{\mathrm{p}}<.05$.

Eine Überprüfung des möglichen Einflusses der unterschiedlichen Ausgangswerte von PA mittels Kovarianzanalyse (siehe Tabelle 16) ergibt keinen signifikanten Effekt des Faktors Gruppe unter der Berücksichtigung der Kovariaten auf die abhängigen Variablen. 
Tabelle 16

Emotionsbezogene Maße: Ergebnisse der Kovarianzanalyse (Kovariate PA/prä-Werte) und der zweifaktoriellen Varianzanalyse mit Messwiederholung (Zeit x Bedingung)

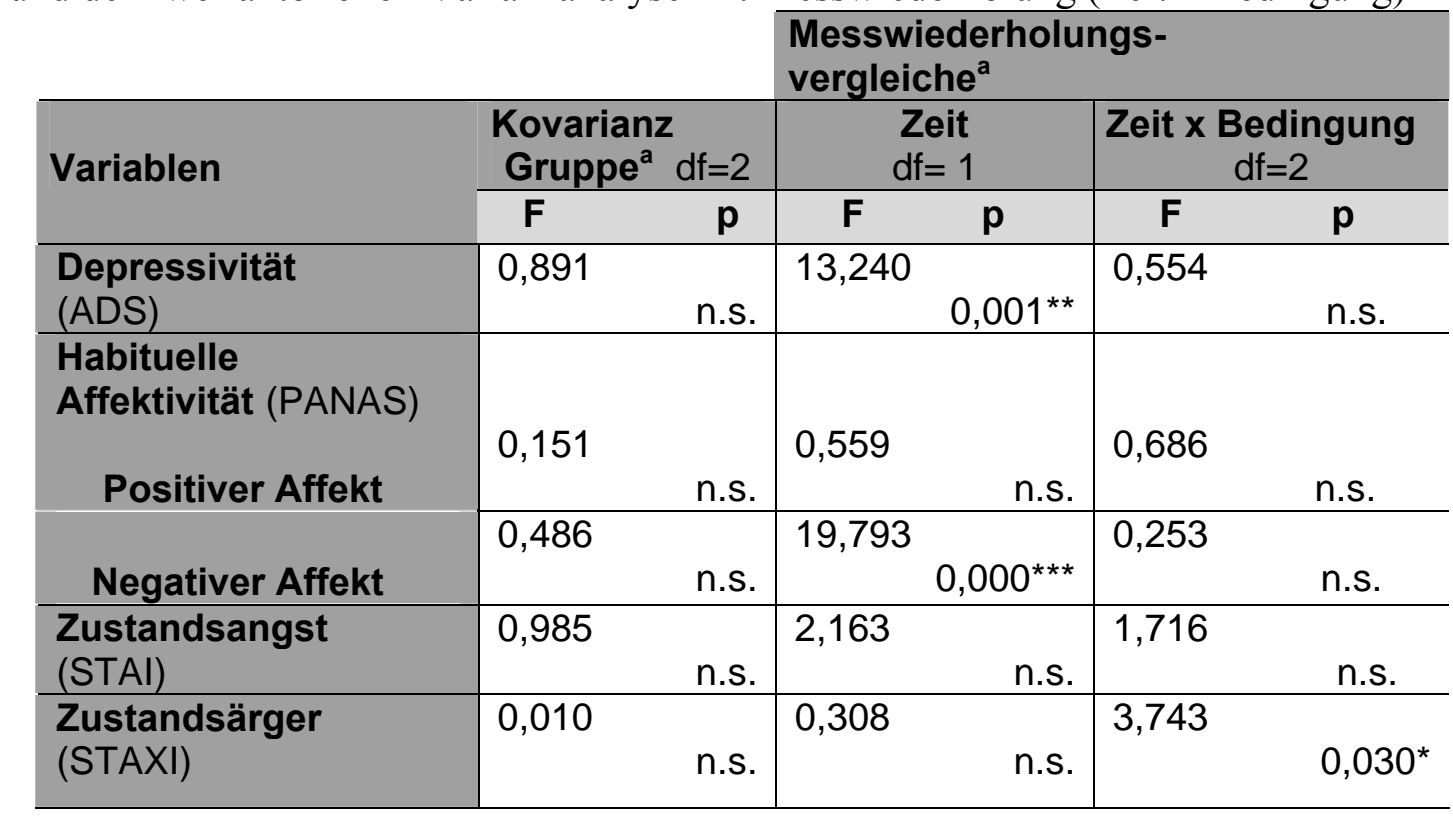

${ }^{a}$ Werte beziehen sich auf $\mathrm{N}=54$, da Missing Data $;{ }^{*} \mathrm{p}<.05,{ }^{* *} \mathrm{p}<.01$.

Neben der Überprüfung eines möglichen Einflusses der Kovariaten zeigt Tabelle 16 einen signifikanten Haupteffekt der Zeit bezüglich zweier Variablen. So nimmt das Ausmaß Negativen Affekts und das der Depressivität für alle Teilnehmer vom Zeitpunkt der Ersterhebung zur Folgeuntersuchung signifikant ab.

Im Rahmen der statistischen Hypothesenprüfung emotionsbezogener Maße zeigt sich ein signifikanter Interaktionseffekt der Faktoren Zeit x Bedingung bezüglich der Variable Zustandsärger. Hinsichtlich dieses Maßes ergibt sich jedoch eine Voraussetzungsverletzung für die Berechnung einer Varianzanalyse, wonach die Fehlervarianz der Variablen Zustandsärger nicht über alle Gruppen hinweg gleich ausfällt (Levéne-Test auf Gleichheit der Fehlervarianz: $F(2,51)=1,895 ; p=0,019)$. 


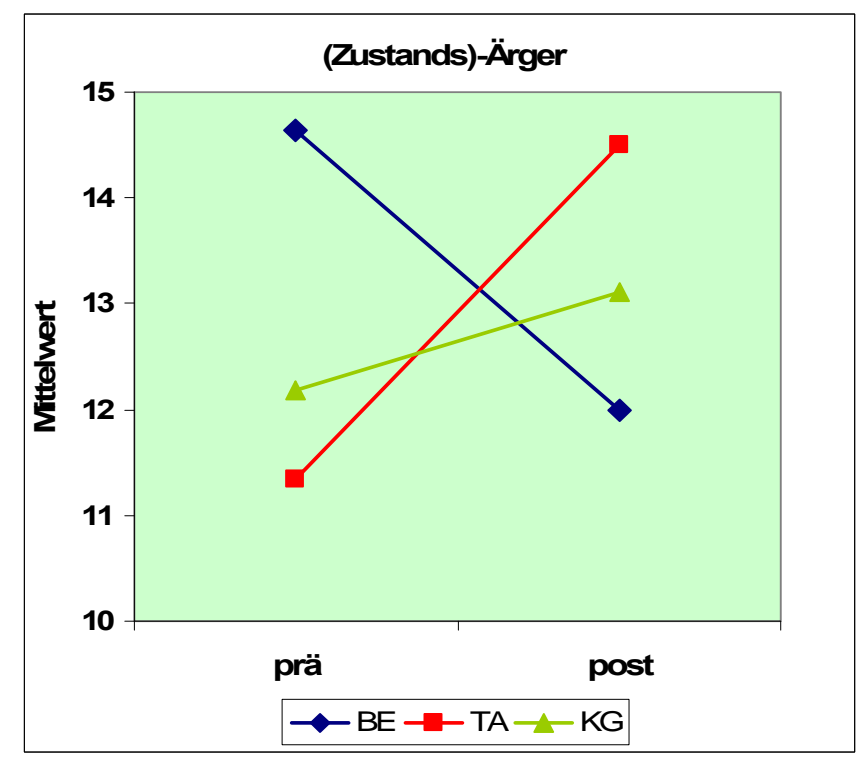

Abbildung 7: Interaktion (Zeit x Bedingung) des Maßes Zustandsärger

In den Gruppen TA und BE zeigt sich eine konträre Reaktion auf die Intervention. Langfristig profitieren vor allem die Teilnehmer der Gruppe BE durch das Schreiben bezüglich ihres Ausmaßes an Ärger, wohingegen in der Gruppe TA eine Zunahme zu erkennen ist. Diese Zunahme ist statistisch signifikant (gepaarter $\mathrm{t}$-Test: $\mathrm{t}=-2,342 ; \mathrm{p}(2$-seitig $)=0,030, \mathrm{df}=19)$, wohingegen die Abnahme an Zustandsärger in der Gruppe BE kein signifikantes Niveau erreicht $(\mathrm{t}=1,506 ; \mathrm{p}(2$-seitig $)=0,148, \mathrm{df}=19)$. Im paarweisen Vergleich zeigt sich ein signifikanter Unterschied zwischen diesen beiden Gruppen (STAXI, F $(1,39)=6,758 ; p=0,013$ ). Für posthoc Vergleiche gilt jedoch einschränkend zu beachten, dass Einzeltestungen das Problem der $\alpha$-Kumulation mit sich bringen. In Bezug auf die Kontrollgruppe bestehen keine relevanten Unterschiede, es zeigen sich zudem keine signifikanten prä-post-Differenzen im Ausmaß von Ärger (gepaarter $\mathrm{t}$-Test: $\mathrm{t}=0,598 ; \mathrm{p}=0,557 ; \mathrm{df}=19$ ).

\subsubsection{Veränderungen in Maßen der Normorientierung und der Führung}

Die Maße der Normorientierung beziehen sich ausschließlich auf Selbstauskunftsmaße, die per Fragebogen vor und nach der Intervention erhoben worden sind. Ein varianzanalytischer Vergleich der Ausgangswerte dieser Maße ergibt zwischen den Gruppen keine signifikanten Unterschiede (Verantwortungsübernahme: $\mathrm{F}(2,57)=1,259$; n.s.; Normorientierung/Skala TLV: 
$\mathrm{F}(2,57)=0,683$; n.s.; Skala ICO: $\mathrm{F}(2,57)=0,288$; n.s.; Moralisches Urteil/Skala SLO: $\mathrm{F}(2,57)=1,0341$; n.s.; Skala SNI: $\mathrm{F}(2,57)=0,367$; n.s. $)$.

Es zeigen sich keinerlei Interaktionseffekte der erhobenen Maße der Normorientierung (vgl. Tabelle 17). Allein bei der Subskala „Straftaten sind lohnend“ des KFN-Fragebogens Moralisches Urteil zeigt sich ein signifikanter Haupteffekt Zeit. Das Ausmaß der Bewertung von Straftaten als „lohnend“ durch die Teilnehmer nimmt demnach im Verlauf der Zeit ab.

Tabelle 17

Maße der Normorientierung: Mittelwerte, Standardabweichungen und Ergebnisse der zwei faktoriellen Varianzanalyse mit Messwiederholung $(\mathrm{N}=60)$

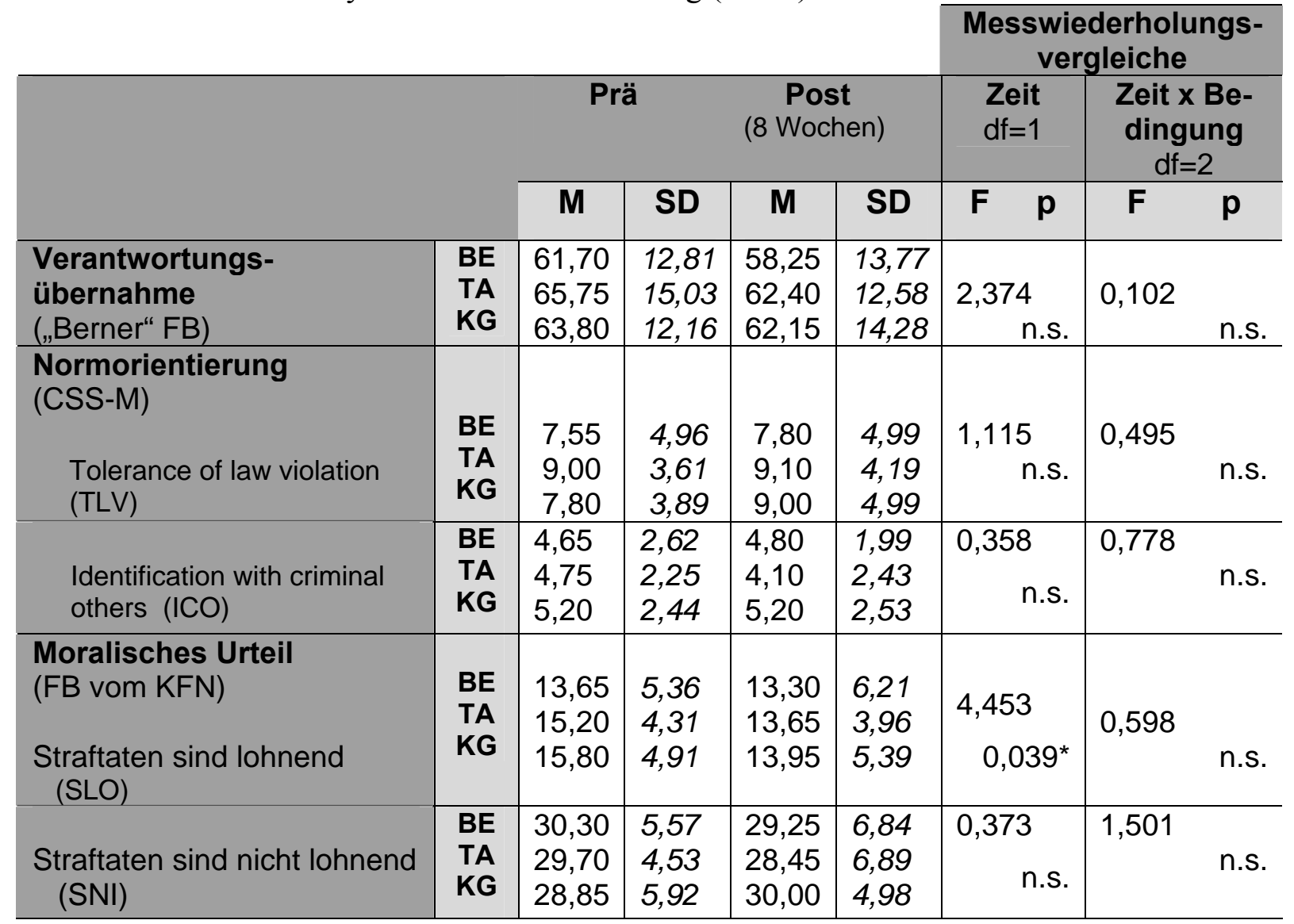

${ }_{\mathrm{p}}<.05$.

Die Maße der Führung beziehen sich ausschließlich auf verhaltensnahe Daten der Teilnehmer, die auf Fremdratings basieren. Hierbei handelt es sich um die monatliche Führungsbenotung (entspricht Schulnoten), die Anzahl der Vermerke und Anhörungen, die der Gefangenenpersonalakte entnommen worden sind und sich auf einen viermonatigen Zeitraum nach der Teilnahme am Schreibprojekt beziehen. 
Tabelle 18

Führungsmaße: Mittelwerte, Standardabweichungen und Ergebnisse der einfaktoriellen Varianzanalyse

\begin{tabular}{|c|c|c|c|c|c|}
\hline & & & & & $\begin{array}{l}\mathbf{V A}^{\mathbf{a}} \\
=2\end{array}$ \\
\hline Variable & & $\mathbf{M}$ & SD & $\mathbf{F}$ & $\mathbf{p}$ \\
\hline Durchschnittliche & BE & 3,13 & 0,53 & & \\
\hline Führungsnote & $\begin{array}{l}\text { TA } \\
\text { KG }\end{array}$ & $\begin{array}{l}3,58 \\
3,54 \\
\end{array}$ & $\begin{array}{l}0,70 \\
0,45 \\
\end{array}$ & 3,734 & $0,030^{*}$ \\
\hline Anzahl & $\mathrm{BE}$ & 1,50 & 1,54 & & \\
\hline Vermerke & $\begin{array}{l}\text { TA } \\
\text { KG }\end{array}$ & $\begin{array}{l}1,10 \\
2,17 \\
\end{array}$ & $\begin{array}{l}1,55 \\
1,92 \\
\end{array}$ & 1,959 & n.s. \\
\hline $\begin{array}{l}\text { Anzahl } \\
\text { Anhörungen }\end{array}$ & $\begin{array}{l}\text { BE } \\
\text { TA } \\
\text { KG }\end{array}$ & $\begin{array}{l}2,93 \\
3,58 \\
3,54\end{array}$ & $\begin{array}{l}0,85 \\
0,70 \\
0,45\end{array}$ & 5,477 & $0,007^{* \star}$ \\
\hline
\end{tabular}

Es ergeben sich varianzanalytisch signifikante Gruppenunterschiede bezüglich der Variablen Führungsbenotung und der Variablen Anhörungen (vgl. Tabelle 18). Werden die drei Maße multivariat betrachtet, ergeben sich ebenfalls signifikante Gruppenunterschiede (Wilks` Lambda: $F=2,483 ; p=0,028)$. Die Variable Vermerke zeigt für sich genommen varianzanalytisch kein signifikantes Ergebnis, wobei davon auszugehen ist, dass es sich bei diesen Maßen nicht um wirklich unabhängige Daten handelt. So können Anhörungen und Vermerke sich durchaus noch rückwirkend negativ auf die monatliche Führungsbenotung auswirken. Es zeigt sich ein signifikanter korrelativer Zusammenhang zwischen der Anzahl der Vermerke und der Führungsbenotung von $\mathrm{r}=.716(\mathrm{p}(2$-seitig $)=0,000)$.

Tabelle 19

Führungsmaße: Ergebnisse der post-hoc Mehrfachvergleiche zwischen den Bedingungen

\begin{tabular}{l|c|c|c}
\cline { 2 - 4 } \multicolumn{1}{c|}{} & \multicolumn{3}{c}{ Tukey-Test } \\
\cline { 2 - 4 } & BE-KG & TA-KG & BE-TA \\
\hline $\begin{array}{l}\text { Durchschnittliche } \\
\text { Führungsnote }\end{array}$ & n.s. & n.s. & $0,042^{*}$ \\
\hline $\begin{array}{l}\text { Anzahl } \\
\text { Vermerke }\end{array}$ & n.s. & n.s. & n.s. \\
\hline $\begin{array}{l}\text { Anzahl } \\
\text { Anhörungen }\end{array}$ & $0,024^{*}$ & n.s. & $0,012^{*}$ \\
\hline
\end{tabular}

* Die mittlere Differenz ist auf der Stufe .05 signifikant

Die Gruppenstatistiken der post-hoc Mehrfachvergleiche zeigen entgegen der Annahme keine signifikant bessere Führungsnoten der Gruppe BE und TA im Vergleich zur Kontrollgruppe (vgl. Tabelle 19). Vielmehr zeigt sich eine signifikant bessere Benotung der Gruppe BE im Vergleich zur Gruppe TA. Hinsichtlich der Anzahl der Anhörungen zeigen sich genau wie 
bei der Führungsbenotung hochsignifikante Unterschiede zwischen den Gruppen BE und TA. Bei den Teilnehmern der Gruppe BE kommt es erwartungskonform zu deutlich weniger Anhörungen. Signifikant weniger Anhörungen hat diese Gruppe auch im Vergleich zu den Teilnehmern der TA, die die höchste Anzahl dieser Art Akteneintragungen aufweist. Die Teilnehmer der beiden Gruppen TA und KG unterscheiden sich entgegen der in der Hypothese formulierten Erwartung nicht in ihrer durchschnittlichen Anzahl der Anhörungen voneinander.

\subsubsection{Einflüsse von Moderatorvariablen}

\section{- Alexithymie}

Es wurde erwartet, dass das individuelle Ausmaß des Persönlichkeitsmerkmals Alexithymie mit den langfristig förderlichen Auswirkungen des ES auf die gesundheitsbezogenen und die psychologischen Maße korreliert. Dabei wurde keine Erwartung über die Richtung dieses Zusammenhangs formuliert. Für die statistische Überprüfung wurden Produkt-MomentKorrelationen zwischen den Differenzwerten der abhängigen Variablen und Alexithymie (Gesamtwert TAS-20) errechnet (vgl. Tabelle 20). Mit den Differenzmaßen der Variablen Zustandsärger und Negativer Affekt zeigen sich signifikante negative Korrelationen von Alexithymie. Hohe Differenzmaße dieser Maße sind gleichbedeutend mit einer langfristigen Abnahme von Ärger und negativer Stimmung. Ein niedriges Ausmaß an Alexithymie geht somit mit einer Abnahme negativen Affekts und Ärger einher. Eine positive signifikante Korrelation ergibt sich im Falle der Variable Vermeidung als eine der drei erhobenen Belastungsreaktionen. Demnach gibt es einen Zusammenhang derart, dass Teilnehmer mit einer hohen Ausprägung an Alexithymie hohe Differenzwerte aufweisen und somit ihr Ausmaß an Vermeidung bezüglich eines belastenden Ereignisses abnimmt. 
Tabelle 20

Korrelationen zwischen der Ausprägung Alexithymie und den Gesundheitsmaßen so wie emotionsbezogener Maße $(\mathrm{N}=40)$

\begin{tabular}{|c|c|c|c|}
\hline Variable & Ergebnismaß & $\mathbf{r}$ & $\begin{array}{c}\mathbf{p} \\
\text { (2-seitig) }\end{array}$ \\
\hline Arztbesuche & Anzahl ( $\Delta$ Prä-Post) & .024 & n.s. \\
\hline Krankheitstage & Anzahl ( $\Delta$ Prä-Post) & -.010 & n.s. \\
\hline Körperl. Beschwerden & BL ( $\Delta$ Prä-Post) & -.157 & n.s. \\
\hline Psychische Symptombelastung & SCL-K9 ( $\Delta$ Prä-Post) & -.015 & n.s. \\
\hline Depressivität & ADS-K ( $\Delta$ Prä-Post) & -.095 & n.s. \\
\hline Positiver Affekt & PANAS ( $\Delta$ Prä-Post) & .094 & n.s. \\
\hline Negativer Affekt & PANAS ( $\Delta$ Prä-Post) & -.359 & $0,023^{*}$ \\
\hline Zustandsangst & STAI ( $\Delta$ Prä-Post) & -.260 & n.s. \\
\hline Zustandsärger & STAXI ( $\Delta$ Prä-Post) & -.497 & $0,001^{* *}$ \\
\hline Intrusion & IES-R-Int ( $\Delta$ Prä-Post) & .280 & n.s. \\
\hline Hyperarousal & IES-R-Hyp ( $\Delta$ Prä-Post) & .283 & n.s. \\
\hline Vermeidung & IES-R-Ver ( $\Delta$ Prä-Post) & .426 & $0,006 * *$ \\
\hline
\end{tabular}

Anmerkungen. Einen Gesamtwert von $\geq 50$ (TAS-20) erzielten $n=25$ (62,5\%), M=53,9, SD=13,45.

Die männliche Normstichprobe weist einen Mittelwert von 40,10 auf, männliche psychiatrische Patienten haben einen Mittelwert von 49,73. Aus „Validierung der deutschen Version der 20-Item Toronto-AlexithymieSkala bei Normalpersonen und psychiatrischen Patienten“, M. Bach et al., 1996, Psychotherapie, Psychosomatik und medizinische Psychologie, 46, p. 26.

${ }^{*} \mathrm{p}<.05, * * \mathrm{p}<.01$.

Entgegen der Hypothese ergeben sich darüber hinaus keine Zusammenhänge von Alexithymie und den gesundheitsbezogenen Maßen Anzahl der Krankheitstage und der Arztbesuche, körperlicher Beschwerden und der psychischen Symptombelastung. Ebenso wenig bestätigt sich ein vermuteter Zusammenhang von Alexithymie mit einer langfristigen Veränderung der psychologischen Maße Depressivität und Zustandsangst.

Eine explorative Untersuchung eines Zusammenhangs zwischen dem Ausmaß an Alexithymie und Normorientierung und Führung ergibt keinerlei signifikante Ergebnisse (vgl. Tabelle 21).

Tabelle 21

Korrelation zwischen der Ausprägung von Alexithymie und der Maße Führung und Normorientierung $(\mathrm{N}=40)$

\begin{tabular}{|c|c|c|c|}
\hline Variable & Ergebnismaß & $\mathbf{r}$ & $\begin{array}{c}\mathbf{p} \\
\text { (2-seitig) }\end{array}$ \\
\hline Führungsbenotung & Durchschnittsnote & .191 & n.s. \\
\hline Verantwortungsübernahme & Berner-FB ( $\Delta$ Prä-Post) & .154 & n.s. \\
\hline Tolerance of Law Violation & CSS-M-TLV ( $\Delta$ Prä-Post) & .155 & n.s. \\
\hline Identification with Criminal Others & CSS-M-ICO ( $\Delta$ Prä-Post) & .029 & n.s. \\
\hline Straftaten sind lohnend & MU-BFS-slo ( $\Delta$ Prä-Post) & .030 & n.s. \\
\hline Straftaten sind nicht lohnend & MU-BFS-sni ( $\Delta$ Prä-Post) & .186 & n.s. \\
\hline
\end{tabular}




\section{- Antisoziale Persönlichkeitsstörung}

Ferner wurde angenommen, dass das Vorhandensein bzw. Fehlen einer Diagnose der Antisozialen Persönlichkeitsstörung (APS, dichotomes Merkmal) sich auf die langfristigen Effekte der Schreibintervention -speziell auf die Veränderungsmaße der Normorientierung und Führung - auswirkt. Das Vorhandensein der Diagnose APS sollte sich auf mögliche Schreibeffekte negativ auswirken. Zur statistischen Überprüfung wurde eine zweifaktorielle Varianzanalyse mit Messwiederholung (doppelt-multivariat), mit einem Faktor APS und einem Messwiederholungsfaktor Tag (prä-post) durchgeführt. Das Messwiederholungsdesign wurde im Vergleich zu der Durchführung einer Kovarianzanalyse (mit dem Merkmal APS als Kovariate ausgewählt), weil es sich als teststärker erweist. Aufgrund zu geringer Zellenbesetzung des Merkmals APS wurde bei der Analyse auf einen Faktor „Gruppe“ verzichtet, so dass lediglich die Ergebnisse der beiden Experimentalgruppen berücksichtigt wurden. Wobei in drei Fällen wegen Minderjährigkeit keine APS-Diagnostik durchgeführt wurde, so dass lediglich die Daten von N=37 in die Analyse eingingen. Statistisch zeigt sich ein signifikanter Interaktionseffekt zwischen den Erhebungszeitpunkten und dem Faktor APS bezüglich der Variablen „Straftaten sind lohnend“.

Tabelle 22

Antisoziale Persönlichkeitsstörung: Mittelwerte, Standardabweichungen und Ergebnisse der multivariaten zweifaktorielle Varianzanalyse mit Messwiederholung mit den Faktoren Zeit (prä und post) und APS (vorhanden/nicht vorhanden)

\begin{tabular}{|c|c|c|c|c|c|c|c|}
\hline \multirow{2}{*}{\multicolumn{2}{|c|}{ Variablen }} & \multicolumn{2}{|c|}{ Prä } & \multicolumn{2}{|c|}{ Post } & \multicolumn{2}{|c|}{ Zeit x APS } \\
\hline & & $\mathbf{M}$ & SD & $\mathbf{M}$ & SD & $\mathbf{F}$ & p \\
\hline $\begin{array}{l}\text { Verantwortungs- } \\
\text { übernahme („Berner“ FB) }\end{array}$ & $\begin{array}{l}\text { APS }^{a} \\
\text { k. APS }\end{array}$ & $\begin{array}{l}64,60 \\
63,05\end{array}$ & $\begin{array}{l}16,13 \\
13,57\end{array}$ & $\begin{array}{l}63,27 \\
59,23\end{array}$ & $\begin{array}{l}15,76 \\
10,70\end{array}$ & 0,248 & n.s. \\
\hline $\begin{array}{l}\text { Normorientierung } \\
\text { Tolerance of law violation } \\
\text { (TLV) }\end{array}$ & $\begin{array}{l}\text { APS } \\
\text { k. APS }\end{array}$ & $\begin{array}{c}10,27 \\
6,45\end{array}$ & $\begin{array}{l}4,06 \\
3,59\end{array}$ & $\begin{array}{c}10,00 \\
7,59\end{array}$ & $\begin{array}{l}4,31 \\
4,72\end{array}$ & 1,139 & n.s. \\
\hline $\begin{array}{l}\text { Identification with criminal } \\
\text { others (ICO) }\end{array}$ & $\begin{array}{l}\text { APS } \\
\text { k. APS }\end{array}$ & $\begin{array}{l}4,93 \\
4,32 \\
\end{array}$ & $\begin{array}{l}2,76 \\
2,21\end{array}$ & $\begin{array}{l}4,80 \\
4,18 \\
\end{array}$ & $\begin{array}{l}2,70 \\
1,92\end{array}$ & 0,000 & n.s. \\
\hline $\begin{array}{l}\text { Moralisches Urteil } \\
\text { Straftaten sind lohnend }\end{array}$ & $\begin{array}{l}\text { APS } \\
\text { k. APS }\end{array}$ & $\begin{array}{l}15,53 \\
13,05\end{array}$ & $\begin{array}{l}4,39 \\
4,54\end{array}$ & $\begin{array}{l}12,93 \\
13,86\end{array}$ & $\begin{array}{l}4,69 \\
5,52\end{array}$ & 5,046 & $0,031^{*}$ \\
\hline Straftaten sind nicht lohnend & $\begin{array}{l}\text { APS } \\
\text { k. APS }\end{array}$ & $\begin{array}{l}29,00 \\
31,50\end{array}$ & $\begin{array}{l}4,19 \\
4,53\end{array}$ & $\begin{array}{l}27,87 \\
30,32\end{array}$ & $\begin{array}{l}7,03 \\
5,88\end{array}$ & 0,001 & n.s. \\
\hline
\end{tabular}

${ }^{a}$ APS $n=15, k$. APS $n=22$

$* \mathrm{p}<.05$. 
Zwischen den Teilnehmern mit der Diagnose APS und solchen ohne zeigen sich konträre Einschätzungen zum Prä- und Postzeitpunkt. Entgegen der Erwartung nimmt die Überzeugung, dass es sich lohnt, Straftaten zu begehen, bei Teilnehmern mit Diagnose zum Zeitpunkt der Folgeerhebung ab und bei denen ohne zu. Einschränkend gilt es zu berücksichtigen, dass bei der multivariaten Berechnung die Voraussetzung der Gleichheit der Kovarianzmatrizen der abhängigen Variablen nicht eingehalten wird (Box-Test auf Gleichheit der Kovarianzmatrizen $\mathrm{F}=1,375 ; \mathrm{p}=0,036)$.

Ein Zusammenhang mit den abhängigen Variablen Führungsbenotung und Anzahl der Anhörungen bzw. Vermerke wurde gesondert geprüft, da in diesen Fällen keine Messwiederholungsdaten vorliegen. Eine statistische Überprüfung mittels Punktbiserialer Korrelationen ergibt keine signifikanten Zusammenhänge mit dem Merkmal APS (vgl. Tabelle 23).

Tabelle 23

Korrelationen zwischen dem Merkmal APS (vorhanden/nicht vorhanden) und Maßen des Führungsverhaltens ( $\mathrm{df}=1)$

\begin{tabular}{l|r|c|c}
\hline Variable & Ergebnismaß & $\mathbf{r}_{\text {pbis }}$ & $\begin{array}{c}\mathbf{p} \\
\text { (2-seitig) }\end{array}$ \\
\hline Führungsbenotung & Durchschnittsnote & .157 & n.s. \\
\hline Vermerke & Anzahl & .087 & n.s. \\
\hline Anhörungen & Anzahl & .197 & n.s. \\
\hline
\end{tabular}

Eine explorative Untersuchung des Zusammenhangs zwischen dem Vorliegen einer APS und möglichen langfristigen Schreibeffekten auf gesundheitsbezogene und Variablen und denen psychischen Befindens ergibt bezüglich der Belastungsreaktionen Intrusion und Hyperarousal signifikante Interaktionen (vgl. Tabelle 24). 
Tabelle 24:

Gesundheitsbezogene Variablen und Emotionsvariablen: Mittelwerte, Standardabweichungen und Ergebnisse der einfaktoriellen Varianzanalyse mit Messwiederholung mit den Faktoren Zeit (prä/post) und APS (vorhanden/nicht vorhanden)

\begin{tabular}{|c|c|c|c|c|c|c|c|}
\hline \multirow{2}{*}{ Variablen } & & \multicolumn{2}{|c|}{ Prä } & \multicolumn{2}{|c|}{ Post } & \multicolumn{2}{|c|}{ Zeit x APS } \\
\hline & & $\mathbf{M}$ & SD & $\mathbf{M}$ & SD & $\mathbf{F}$ & $p$ \\
\hline Anzahl Krankheitstage & $\begin{array}{l}\text { APS }^{\mathrm{a}} \\
\text { k. APS }\end{array}$ & $\begin{array}{l}2,13 \\
1,20\end{array}$ & $\begin{array}{l}3,78 \\
2,38\end{array}$ & $\begin{array}{l}3,07 \\
4,05\end{array}$ & $\begin{array}{l}6,54 \\
5,96\end{array}$ & 0,78 & n.s. \\
\hline Anzahl Arztbesuche & $\begin{array}{l}\text { APS } \\
\text { k. APS }\end{array}$ & $\begin{array}{l}1,73 \\
1,60\end{array}$ & $\begin{array}{l}1,39 \\
2,26\end{array}$ & $\begin{array}{l}2,27 \\
1,90\end{array}$ & $\begin{array}{l}3,17 \\
2,22\end{array}$ & 0,44 & n.s. \\
\hline Körperl. Beschwerden (BL) & $\begin{array}{l}\text { APS } \\
\text { k. APS }\end{array}$ & $\begin{array}{l}19,53 \\
16,75\end{array}$ & $\begin{array}{c}12,14 \\
9,73\end{array}$ & $\begin{array}{l}15,87 \\
16,95\end{array}$ & $\begin{array}{c}9,50 \\
13,01\end{array}$ & 1,319 & n.s. \\
\hline $\begin{array}{l}\text { Psychische Symptombelastung } \\
\text { (SLC-K9) }\end{array}$ & $\begin{array}{l}\text { APS } \\
\text { k. APS }\end{array}$ & $\begin{array}{l}1,25 \\
1,15\end{array}$ & $\begin{array}{l}0,73 \\
0,85\end{array}$ & $\begin{array}{l}1,05 \\
1,04\end{array}$ & $\begin{array}{l}0,65 \\
0,87\end{array}$ & 0,118 & n.s. \\
\hline Depressivität (ADS-K) & $\begin{array}{l}\text { APS } \\
\text { k. APS }\end{array}$ & $\begin{array}{l}18,40 \\
14,40\end{array}$ & $\begin{array}{l}7,80 \\
8,56\end{array}$ & $\begin{array}{l}12,93 \\
13,50\end{array}$ & $\begin{array}{c}9,61 \\
11,05\end{array}$ & 1,698 & n.s. \\
\hline Positiver Affekt (PANAS) & $\begin{array}{l}\text { APS } \\
\text { k. APS }\end{array}$ & $\begin{array}{l}27,07 \\
28,05\end{array}$ & $\begin{array}{l}7,89 \\
6,68\end{array}$ & $\begin{array}{l}27,40 \\
27,95\end{array}$ & $\begin{array}{l}+1,00 \\
8,79 \\
7,84\end{array}$ & 0,030 & n.s. \\
\hline Negativer Affekt (PANAS) & $\begin{array}{l}\text { APS } \\
\text { k. APS }\end{array}$ & $\begin{array}{l}22,60 \\
22,20\end{array}$ & $\begin{array}{l}7,25 \\
6,68\end{array}$ & $\begin{array}{l}19,47 \\
18,40\end{array}$ & $\begin{array}{l}8,16 \\
6,27\end{array}$ & 0,030 & n.s. \\
\hline Zustandsangst (STAI) & $\begin{array}{l}\text { APS } \\
\text { k. APS }\end{array}$ & $\begin{array}{l}44,33 \\
41,35\end{array}$ & $\begin{array}{c}11,48 \\
8,66\end{array}$ & $\begin{array}{l}41,00 \\
41,05\end{array}$ & $\begin{array}{l}9,54 \\
7,98\end{array}$ & 0,087 & n.s. \\
\hline Zustandsärger (STAXI) & $\begin{array}{l}\text { APS } \\
\text { k. APS }\end{array}$ & $\begin{array}{l}15,53 \\
11,40\end{array}$ & $\begin{array}{l}6,32 \\
2,11\end{array}$ & $\begin{array}{l}13,46 \\
13,30\end{array}$ & $\begin{array}{l}5,39 \\
5,96\end{array}$ & 2,531 & n.s. \\
\hline Intrusion (IES-R) & $\begin{array}{l}\text { APS } \\
\text { k. APS }\end{array}$ & $\begin{array}{c}14,00 \\
9,40\end{array}$ & $\begin{array}{l}9,83 \\
9,94\end{array}$ & $\begin{array}{l}4,80 \\
7,00\end{array}$ & $\begin{array}{l}5,00 \\
9,11\end{array}$ & 7,229 & $0,011^{\star}$ \\
\hline Hyperarousal (IES-R) & $\begin{array}{l}\text { APS } \\
\text { k. APS }\end{array}$ & $\begin{array}{c}12,40 \\
7,55\end{array}$ & $\begin{array}{l}9,02 \\
7,73\end{array}$ & $\begin{array}{l}5,40 \\
6,25\end{array}$ & $\begin{array}{l}6,23 \\
6,96\end{array}$ & 6,993 & $0,012^{\star}$ \\
\hline Vermeidung (IES-R) & $\begin{array}{l}\text { APS } \\
\text { k. APS }\end{array}$ & $\begin{array}{l}15,33 \\
11,05\end{array}$ & $\begin{array}{l}9,79 \\
6,46\end{array}$ & $\begin{array}{l}9,87 \\
8,65\end{array}$ & $\begin{array}{l}8,53 \\
7,30\end{array}$ & 0,936 & n.s. \\
\hline
\end{tabular}

$* \mathrm{p}<.05$.

${ }^{\mathrm{a}}$ APS $n=15, \mathrm{k}$. APS $n=20$.

Gepaarte t-Tests zeigen, dass insbesondere diejenigen Teilnehmer eine Verbesserung dieser Belastungsreaktionen aufweisen, die die Kriterien einer APS erfüllen (Intrusion: $t=3,532 ; p=$ 0,003; Hyperarousal: $\mathrm{t}=3,125 ; \mathrm{p}=0,007)$.

\section{- Ausgewählte Essaymerkmale}

Es wurde ferner erwartet, dass die Häufigkeit, mit der Worte bestimmter Wortkategorien in den Essays benutzt wurden, mit den langfristig förderlichen Auswirkungen des ES auf die gesundheitsbezogenen und psychologischen Maße sowie Maßen der Führung und Normorientierung zusammenhängt. Im einzelnen handelt es sich hierbei um die Kategorien positive und negative Emotionsworte, Worte kognitiver Mechanismen und Worte sozialer Prozesse. 
Die computerbasierte Auswertung mittels LIWC zählt nach eingelesenem Wörterbuch Worte dieser Kategorien und gibt das prozentuale Verhältnis zur Gesamtwortzahl der Essays an. In den beiden erstgenannten Kategorie werden Worte positiver Gefühle, Optimismus und Energie oder aber negativer Gefühle von Angst, Wut, Furcht oder Traurigkeit gezählt. Kognitive Prozesse spiegeln sich in Worten von Kausalität, Einsicht, Diskrepanz, Inhibition, Eventualität und Bestimmtheit wider. In der Kategorie der sozialen Prozesse finden sich Ausdrücke der Kommunikation, Bezüge auf andere Personen, Freunde und Familie.

Wie aus Tabelle 25 ersichtlich wird, weist die Häufigkeit positiver Emotionsworte lediglich mit dem Ergebnismaß Führungsbenotung eine signifikante Korrelation auf. Es besteht somit ein - wenn auch nur geringer - Zusammenhang dergestalt, dass die Benotung der Führung langfristig besser ausfällt, je größer die Häufigkeit von Ausdrücken positiver Emotionalität in den Essays ist. Ferner zeigt sich eine positive signifikante Korrelation in der Kategorie Kognitionsworte mit dem Ausmaß an Verantwortungsübernahme bezüglich der eigenen Straftat. Da höhere Differenzwerte dieses Maßes eine Abnahme an Verantwortungsablehnung widerspiegeln, besagt diese Korrelation, dass solche Teilnehmer, die zu einer gesteigerten Verantwortungsübernahme im prä-post-Vergleich gelangen, in ihren Essays mehr Worte kognitiver Mechanismen verwendet haben.

Bezüglich der Anzahl der Worte, die soziale Prozesse signalisieren ergeben sich keinerlei Zusammenhänge mit den langfristigen Ergebnismaßen.

\subsection{Explorative Untersuchung weiterer potentieller Einflussgrößen}

\subsubsection{Kurzfristiger Affekt}

Explorativ wurde untersucht, inwieweit ein Zusammenhang zwischen kurzfristigen und langfristigen Auswirkungen des Schreibens in den Experimentalgruppen besteht. Eine statistische Überprüfung mittels Produkt-Moment-Korrelationen ergibt zwei signifikante Zusammenhänge des Maßes kurzfristiger Positiver Affekt (vgl. Tabelle 26). Mit ihm korrelieren die Differenzwerte der Belastungsreaktionen Intrusion und Hyperarousal. Teilnehmer, die direkt nach der Schreibsitzung eine bessere Stimmung aufwiesen, zeigen demnach langfristig eine stärkere Abnahme der Symptome Intrusionen und Hyperarousal. Zwischen kurzfristigem Negativen Affekt und der Variablen Verantwortungsübernahme bzw. -ablehnung (für die 
eigene Straffälligkeit) findet sich eine signifikante negative Korrelation, wonach ein hohes $\mathrm{Maß}$ kurzfristiger schlechter Stimmung mit einer langfristigen Abnahme an Verantwortungsübernahme einhergeht.

Tabelle 26:

Korrelation zwischen kurzfristigen Affektmaßen und langfristigen Ergebnismaßen (Maße der Normorientierung/Führung, der Gesundheit und emotionsbezogener Maße, Belastungsreaktionen ), $\mathrm{N}=40$

\begin{tabular}{|c|c|c|c|c|c|}
\hline & \multicolumn{4}{|c|}{ Kurzfristiger Affekt } \\
\hline \multirow[t]{2}{*}{ Variable } & \multirow[t]{2}{*}{ Ergebnismaß } & \multicolumn{2}{|c|}{ PA } & \multicolumn{2}{|c|}{ NA } \\
\hline & & $\mathbf{r}$ & $p^{a}$ & $\mathbf{r}$ & $p^{a}$ \\
\hline Führungsbenotung & Durchschnittsnote & -.044 & n.s. & -.289 & n.s. \\
\hline Verantwortungsübernahme & Berner-FB ( $\Delta$ Prä-Post) & .090 & n.s. & -.319 & $0,039 *$ \\
\hline Tolerance of Law Violation & CSS-M-TLV ( $\Delta$ Prä-Post) & -.053 & n.s. & -.038 & n.s. \\
\hline Identification with Criminal Others & CSS-M-ICO ( $\Delta$ Prä-Post) & .173 & n.s. & -.110 & n.s. \\
\hline Straftaten sind lohnend & MU-BFS-slo ( $\Delta$ Prä-Post) & .004 & n.s. & .064 & n.s. \\
\hline Straftaten sind nicht lohnend & MU-BFS-sni ( $\Delta$ Prä-Post) & .069 & n.s. & .074 & n.s. \\
\hline Arztbesuche & Anzahl ( $\Delta$ Prä-Post) & -.126 & n.s. & -.164 & n.s. \\
\hline Krankheitstage & Anzahl ( $\Delta$ Prä-Post) & -.195 & n.s. & -.236 & n.s. \\
\hline Körperl. Beschwerden & BL ( $\Delta$ Prä-Post) & -.299 & n.s. & -.070 & n.s. \\
\hline Psychische Symptombelastung & SCL-K9 ( $\Delta$ Prä-Post) & -.100 & n.s. & -.246 & n.s. \\
\hline Depressivität & ADS-K ( $\Delta$ Prä-Post) & -.110 & n.s. & -.185 & n.s. \\
\hline Positiver Affekt & PANAS ( $\Delta$ Prä-Post) & -.107 & n.s. & .205 & n.s. \\
\hline Negativer Affekt & PANAS ( $\Delta$ Prä-Post) & -.131 & n.s. & -.070 & n.s. \\
\hline Zustandsangst & STAI ( $\Delta$ Prä-Post) & .058 & n.s. & -.014 & n.s. \\
\hline Zustandsärger & STAXI ( $\Delta$ Prä-Post) & -.076 & n.s. & .103 & n.s. \\
\hline Intrusionen & IES-R-Int ( $\Delta$ Prä-Post) & .523 & $0,001^{* *}$ & -.092 & n.s. \\
\hline Hyperarousal & IES-R-Hyp ( $\Delta$ Prä-Post) & .320 & $0,044^{*}$ & -.082 & n.s. \\
\hline Vermeidung & IES-R-Ver ( $\Delta$ Prä-Post) & .122 & n.s. & .081 & n.s. \\
\hline
\end{tabular}

\subsubsection{Soziodemografische Merkmale}

Es wurde untersucht, ob ausgewählte soziodemografische Merkmale in Zusammenhang mit langfristigen Effekten des ES stehen. Für die abhängige Variable Alter $(\mathrm{N}=40)$ zeigt sich für die Experimentalgruppen keinerlei relevante Zusammenhänge mit den Differenzwerten der verschiedenen Ergebnismaße (vgl. Tabelle 27).

Tabelle 27 
Korrelation zwischen den Maßen Alter/Schulabschluss und den langfristigen Ergebnismaßen (Maße der Normorientierung/Führung, der Gesundheit und emotionsbezogener Maße, Belastungsreaktionen, $\mathrm{N}=40$ )

\begin{tabular}{|c|c|c|c|c|c|}
\hline \multirow[t]{2}{*}{ Variable } & \multirow[t]{2}{*}{ Ergebnismaß } & \multicolumn{2}{|c|}{ Alter } & \multicolumn{2}{|c|}{$\begin{array}{c}\text { Schul - } \\
\text { abschluß }\end{array}$} \\
\hline & & $\mathbf{r}$ & $p^{a}$ & $\varepsilon$ & $p^{b}$ \\
\hline Führungsbenotung & Durchschnittsnote & -.278 & n.s. & .286 & n.s. \\
\hline Verantwortungsübernahme & Berner-FB ( $\Delta$ Prä-Post) & -.055 & n.s. & .383 & n.s. \\
\hline Tolerance of Law Violation & CSS-M-TLV ( $\Delta$ Prä-Post) & -.228 & n.s. & .324 & n.s. \\
\hline Identification with Criminal Others & CSS-M-ICO ( $\Delta$ Prä-Post) & -.190 & n.s. & .211 & n.s. \\
\hline Straftaten sind lohnend & MU-BFS-slo ( $\Delta$ Prä-Post) & -.276 & n.s. & .168 & n.s. \\
\hline Straftaten sind nicht lohnend & MU-BFS-sni ( $\Delta$ Prä-Post) & .055 & n.s. & .256 & n.s. \\
\hline Arztbesuche & Anzahl ( $\Delta$ Prä-Post) & .131 & n.s. & .073 & n.s. \\
\hline Krankheitstage & Anzahl ( $\Delta$ Prä-Post) & .075 & n.s. & .168 & n.s. \\
\hline Körperl. Beschwerden & BL ( $\Delta$ Prä-Post) & -.076 & n.s. & .278 & n.s. \\
\hline Psychische Symptombelastung & SCL-K9 ( $\Delta$ Prä-Post) & -.272 & n.s. & .438 & $0,018^{*}$ \\
\hline Depressivität & ADS-K ( $\Delta$ Prä-Post) & -.125 & n.s. & .475 & $0,006^{\star \star}$ \\
\hline Positiver Affekt & PANAS ( $\Delta$ Prä-Post) & .115 & n.s. & .302 & n.s. \\
\hline Negativer Affekt & PANAS ( $\Delta$ Prä-Post) & -.037 & n.s. & .477 & $0,007^{\star *}$ \\
\hline Zustandsangst & STAI ( $\Delta$ Prä-Post) & .305 & n.s. & .315 & n.s. \\
\hline Zustandsärger & STAXI ( $\Delta$ Prä-Post) & -.068 & n.s. & .119 & n.s. \\
\hline Intrusionen & IES-R-Int ( $\Delta$ Prä-Post) & .050 & n.s. & .161 & n.s. \\
\hline Hyperarousal & IES-R-Hyp ( $\Delta$ Prä-Post) & .083 & n.s. & .143 & n.s. \\
\hline Vermeidung & IES-R-Ver ( $\Delta$ Prä-Post) & .130 & n.s. & .172 & n.s. \\
\hline
\end{tabular}

Anmerkungen. Keinen Schulabschluss haben $n=23$, Sonderschulabschluss haben $n=3$, Hauptschulabschluss haben $\mathrm{n}=13$, Realschulabschluss $\mathrm{n}=1$.

${ }^{\mathrm{a}}$ zweiseitig; ${ }^{\mathrm{b}}$ varianzanalytische Signifikanzprüfung des Eta-Koeffizienten; ${ }^{*} \mathrm{p}<.05,{ }^{* *} \mathrm{p}<.01$.

Die Variable Schulabschluss (differenziert nach Schulform in aufsteigender Kodierung) und ihr Zusammenhang mit den verschiedenen langfristigen Ergebnismaßen wird mittels Korrelationsverhältnis Eta (kategoriales Merkmal als UV, metrische Variablen als AV) ausgedrückt. Es zeigt sich eine statistisch signifikante Beziehung zwischen Schulabschluss und dem Gesundheitsmaß Psychische Symptombelastung ( $\triangle$ Prä-Post, SCL-K9). Ein (höherer) Schulabschluss des Teilnehmers hängt demnach mit abnehmender Symptombelastung (z.B. geringerer Ängstlichkeit, soziale Unsicherheit, Zwanghaftigkeit) zusammen. Auch mit zwei psychologischen Variablen zeigen sich signifikante Korrelationen: mit Depressivität ( $\Delta$ Prä-Post, ADS-K) und Negativem Affekt ( $\Delta$ Prä-Post, PANAS). Es lässt sich feststellen, dass die mittleren Differenzwerte dieser Variablen, die ein reduzierten depressiven/negativen Affekt ausdrücken, für die Kategorien „kein Schulabschluss“ und „Hauptschulabschluss“ höher sind als für die Kategorie „Sonderschulabschluss“ 


\subsubsection{Kriminogene und haftspezifische Faktoren}

Soweit Merkmale im Rahmen dieser Studie berücksichtigt wurden, die sich statistisch als rückfallgefährdend bzw. als protektiv erwiesen haben (z.B. Alkohol- und Drogenkonsum während der Inhaftierung oder Alter zum Zeitpunkt der ersten Straftat), soll ein möglicher differentieller Einfluss auf die Differenzwerte der Ergebnisvariablen in den Experimentalgruppen $(\mathrm{N}=40)$ untersucht werden. Aber auch in Bezug auf andere für diese Stichprobe spezifische Faktoren wie z.B. die Straflänge gilt es, ihren möglichen Einfluss auf die Ergebnisse zu überprüfen (vgl. Tabelle 28).

Zwischen Alter bei der ersten Straftat und den Differenzwerte der drei Kategorien von Variablen zeigen sich keine relevanten Zusammenhänge. Es ergibt sich eine signifikante negative Korrelation mit dem Maß Führungsbenotung und der Straflänge. Teilnehmer, die lange Haftstrafen zu verbüßen haben, weisen bessere Noten auf und zeigen zudem eine Zunahme an Normorientierung.

Tabelle 28

Korrelation zwischen den Maßen Straflänge/Alter bei erster Straffälligkeit und den Differenzwerten der Ergebnismaße (Maße der Normorientierung/Führung, der Gesundheit und emotionsbezogener Maße, Belastungsreaktionen, $\mathrm{N}=40$ )

\begin{tabular}{|c|c|c|c|c|c|}
\hline \multirow[t]{2}{*}{ Variable } & \multirow[t]{2}{*}{ Ergebnismaß } & \multicolumn{2}{|c|}{ Straflänge } & \multicolumn{2}{|c|}{$\begin{array}{c}\text { Alter bei } \\
\text { erster } \\
\text { Straffälligkeit }\end{array}$} \\
\hline & & $\mathbf{r}$ & $p^{a}$ & $\mathbf{r}$ & $p^{a}$ \\
\hline Führungsbenotung & Durchschnittsnote & -.336 & $0,020^{*}$ & .041 & n.s. \\
\hline Verantwortungsübernahme & Berner-FB ( $\Delta$ Prä-Post) & .133 & n.s. & .218 & n.s. \\
\hline Tolerance of Law Violation & CSS-M-TLV ( $\Delta$ Prä-Post) & .325 & $0,041^{*}$ & -.017 & n.s. \\
\hline Identification with Criminal Others & CSS-M-ICO ( $\Delta$ Prä-Post) & .203 & n.s. & -.221 & n.s. \\
\hline Straftaten sind lohnend & MU-BFS-slo ( $\Delta$ Prä-Post) & .368 & $0,014^{*}$ & -.201 & n.s. \\
\hline Straftaten sind nicht lohnend & MU-BFS-sni ( $\Delta$ Prä-Post) & .119 & n.s. & -.023 & n.s. \\
\hline Arztbesuche & Anzahl ( $\Delta$ Prä-Post) & -.278 & n.s. & .157 & n.s. \\
\hline Krankheitstage & Anzahl ( $\Delta$ Prä-Post) & -.372 & $0,018^{\star}$ & -.061 & n.s. \\
\hline Körperl. Beschwerden & BL ( $\Delta$ Prä-Post) & .248 & n.s. & -.281 & n.s. \\
\hline Psychische Symptombelastung & SCL-K9 ( $\Delta$ Prä-Post) & -.148 & n.s. & -.214 & n.s. \\
\hline Depressivität & ADS-K ( $\Delta$ Prä-Post) & .127 & n.s. & -.186 & n.s. \\
\hline Positiver Affekt & PANAS ( $\Delta$ Prä-Post) & -.139 & n.s. & .284 & n.s. \\
\hline Negativer Affekt & PANAS ( $\Delta$ Prä-Post) & .233 & n.s. & -.210 & n.s. \\
\hline Zustandsangst & STAI ( $\Delta$ Prä-Post) & -.115 & n.s. & -.271 & n.s. \\
\hline Zustandsärger & STAXI ( $\Delta$ Prä-Post) & .099 & n.s. & .058 & n.s. \\
\hline
\end{tabular}




\begin{tabular}{l|r|c|c|c|c}
\hline Intrusion & IES-R-Int $(\Delta$ Prä-Post) & -.066 & n.s. & -.078 & n.s. \\
\hline Hyperarousal & IES-R-Hyp $(\Delta$ Prä-Post) & -.214 & n.s. & -.032 & n.s. \\
\hline Vermeidung & IES-R-Ver $(\Delta$ Prä-Post) & .057 & n.s. & .260 & n.s. \\
\hline
\end{tabular}

${ }^{\mathrm{a}}$ zweiseitig; $* \mathrm{p}<.05$.

Zum ersten Erhebungszeitpunkt wurden die Teilnehmer gefragt, worin sie die Gründe für ihre Straffälligkeit sehen. Die Antworten konnten grob neun Kategorien zugeordnet werden: (1)Alkohol-/Drogenkonsum, (2) Spaß/,Kick“, (3) Langeweile, (4) eigene Suchtproblematik, (5) Geldsorgen/Schulden, (6) äußere Verlockung, (7) Arbeitslosigkeit, (8) deviante Peergroup, (9) andere Umstände. Die in diesen Antworten zum Ausdruck kommenden Ursachenzuschreibungen wurden zwei Kategorien zugeteilt (dichotomes Merkmal), nämlich in die innerhalb der Person (1-4) oder in die außerhalb der Person des Täters (5-9) liegenden Gründe. Dabei sahen 15 Teilnehmer die Gründe in der eigenen Person und 25 außerhalb der eigenen Person liegen. Die Zuordnung der Schuld für die Straffälligkeit in Schuldzuweisung konnte ebenfalls in zwei Kategorien unterteilt werden, nämlich zur eigenen Person (,,ich selbst bin schuld“ bzw. „wir, die Tätergruppe“) und zu äußeren Umständen (Drogen- Alkoholkonsum, Opfer, andere Umstände). 27 Personen gaben sich selbst die Schuld für die Straffälligkeit, 13 schrieben sie anderen bzw. äußeren Ursachen zu.

Wie aus Tabelle 29 hervorgeht, zeigt sich ein Zusammenhang zwischen der Zuschreibung der Ursachen zu außerhalb der Person liegenden Umständen und der langfristigen Abnahme positiven Affekts. Die Schuldzuweisung zu äußeren Umständen geht mit einer langfristigen Abnahme negativen Affekts einher. 
Tabelle 29

Korrelation zwischen den Angaben für die Gründe bzw. Schuld der Straffälligkeit und den Differenzwerten der Ergebnismaße (Maße der Normorientierung/Führung, der Gesundheit und emotionsbezogener Maße, Belastungsreaktionen; $\mathrm{N}=40 ; \mathrm{df}=1$ )

\begin{tabular}{|c|c|c|c|c|c|}
\hline \multirow[t]{2}{*}{ Variable } & \multirow[t]{2}{*}{ Ergebnismaß } & \multicolumn{2}{|c|}{$\begin{array}{c}\text { Gründe für } \\
\text { Straffälligkeit }\end{array}$} & \multicolumn{2}{|c|}{$\begin{array}{c}\text { Schuld für } \\
\text { Straffälligkeit }\end{array}$} \\
\hline & & $r_{\text {pbis }}$ & $p^{a}$ & $\mathbf{r}_{\mathrm{pbis}}$ & $p^{a}$ \\
\hline Führungsbenotung & Durchschnittsnote & .002 & n.s. & .170 & n.s. \\
\hline Verantwortungsübernahme & Berner-FB ( $\Delta$ Prä-Post) & .169 & n.s. & .070 & n.s. \\
\hline Tolerance of Law Violation & CSS-M-TLV ( $\Delta$ Prä-Post) & .259 & n.s. & .042 & n.s. \\
\hline Identification with Criminal Others & CSS-M-ICO ( $\Delta$ Prä-Post) & .006 & n.s. & .147 & n.s. \\
\hline Straftaten sind lohnend & MU-BFS-slo ( $\Delta$ Prä-Post) & .046 & n.s. & .209 & n.s. \\
\hline Straftaten sind nicht lohnend & MU-BFS-sni ( $\Delta$ Prä-Post) & .012 & n.s. & .099 & n.s. \\
\hline Arztbesuche & Anzahl ( $\Delta$ Prä-Post) & .004 & n.s. & .205 & n.s. \\
\hline Krankheitstage & Anzahl ( $\Delta$ Prä-Post) & .089 & n.s. & .169 & n.s. \\
\hline Körperl. Beschwerden & BL ( $\Delta$ Prä-Post) & .011 & n.s. & .025 & n.s. \\
\hline Psychische Symptombelastung & SCL-K9 ( $\Delta$ Prä-Post) & .078 & n.s. & .220 & n.s. \\
\hline Depressivität & ADS-K ( $\Delta$ Prä-Post) & .069 & n.s. & .130 & n.s. \\
\hline Positiver Affekt & PANAS ( $\Delta$ Prä-Post) & .334 & $0,043^{\star}$ & .128 & n.s. \\
\hline Negativer Affekt & PANAS ( $\Delta$ Prä-Post) & .190 & n.s. & .387 & $0,014^{*}$ \\
\hline Zustandsangst & STAI ( $\Delta$ Prä-Post) & .207 & n.s. & .016 & n.s. \\
\hline Zustandsärger & STAXI ( $\Delta$ Prä-Post) & .125 & n.s. & .126 & n.s. \\
\hline Intrusion & IES-R-Int ( $\Delta$ Prä-Post) & .002 & n.s. & .043 & n.s. \\
\hline Hyperarousal & IES-R-Hyp ( $\Delta$ Prä-Post) & .135 & n.s. & .119 & n.s. \\
\hline Vermeidung & IES-R-Ver ( $\Delta$ Prä-Post) & .199 & n.s. & .285 & n.s. \\
\hline
\end{tabular}

${ }^{\mathrm{a}}$ zweiseitig

$* \mathrm{p}<.05$.

Das Merkmal Täterschaft (Einzel- oder Gruppentäter) steht in relevanten Zusammenhang mit Ergebnisvariablen der Normorientierung (vgl. Tabelle 30). Hier zeigen sich signifikante Korrelationen, die bedeuten, dass Gruppentäterschaft mit verbesserter Normorientierung bzw. erhöhter Verantwortungsübernahme einhergeht. Während der Haft Alkohol- und Drogen konsumiert zu haben (dichotomes Merkmal: Konsum vorhanden/nicht vorhanden im Verlauf der letzten sechs Wochen), gaben 15 der Teilnehmer an. Dieses Merkmal steht mit keinerlei langfristiger Ergebnismaße in relevanter Beziehung. Jugendliche, die bezüglich des Zeitraums von sechs Wochen vor der Haft Alkohol und Drogenkonsum (dichotomes Merkmal) angegeben haben $(n=31)$, zeigen eine stärkere Verantwortungszunahme und eine Abnahme an psychischer Symptombelastung. 
Tabelle 30

Korrelation zwischen Täterschaft/Alkohol- und Drogenkonsum und den Differenzwerten der Ergebnismaße (Maße der Normorientierung/Führung, der Gesundheit und emotionsbezogener Maße, Belastungsreaktionen, $\mathrm{N}=40$ )

\begin{tabular}{|c|c|c|c|c|c|c|}
\hline \multirow[b]{2}{*}{ Variable } & \multicolumn{2}{|c|}{ Täterschaft } & \multicolumn{4}{|c|}{\begin{tabular}{c}
\multicolumn{2}{c}{ Alkohol- u. Drogenkonsum } \\
während Haft \\
\end{tabular}} \\
\hline & $\mathbf{r}_{\text {pbis }}$ & $p^{a}$ & $\mathbf{r}_{\text {pbis }}$ & $p^{a}$ & $\mathbf{r}_{\text {pbis }}$ & $p^{a}$ \\
\hline Führungsbenotung & .070 & n.s. & .054 & n.s. & .086 & n.s. \\
\hline Verantwortungsübernahme & .340 & $0,032^{*}$ & .067 & n.s. & .358 & $0,014^{*}$ \\
\hline Tolerance of Law Violation & .370 & $0,019 *$ & .020 & n.s. & .123 & n.s. \\
\hline Identification with Criminal Others & .441 & $0,004^{* \star}$ & .040 & n.s. & .112 & n.s. \\
\hline Straftaten sind lohnend & .168 & n.s. & .095 & n.s. & .031 & n.s. \\
\hline Straftaten sind nicht lohnend & .140 & n.s. & .075 & n.s. & .018 & n.s. \\
\hline Arztbesuche & .082 & n.s. & .114 & n.s. & .044 & n.s. \\
\hline Krankheitstage & .132 & n.s. & .054 & n.s. & .022 & n.s. \\
\hline Körperl. Beschwerden & .056 & n.s. & .148 & n.s. & .191 & n.s. \\
\hline Psychische Symptombelastung & .046 & n.s. & .118 & n.s. & .335 & $0,035^{\star}$ \\
\hline Depressivität & .188 & n.s. & .020 & n.s. & .067 & n.s. \\
\hline Positiver Affekt & .111 & n.s. & .105 & n.s. & .020 & n.s. \\
\hline Negativer Affekt & .147 & n.s. & .009 & n.s. & .182 & n.s. \\
\hline Zustandsangst & .207 & n.s. & .021 & n.s. & .126 & n.s. \\
\hline Zustandsärger & .105 & n.s. & .087 & n.s. & .083 & n.s. \\
\hline Intrusion & .145 & n.s. & .202 & n.s. & .132 & n.s. \\
\hline Hyperarousal & .088 & n.s. & .093 & n.s. & .010 & n.s. \\
\hline Vermeidung & .249 & n.s. & .015 & n.s. & .080 & n.s. \\
\hline
\end{tabular}

${ }^{\mathrm{a}}$ zweiseitig

$* \mathrm{p}<.05, * * \mathrm{p}<.01$.

\section{Diskussion}

\subsection{Anwendbarkeit und Wirksamkeit Expressiven Schreibens im Jugendvollzug}

Zunächst kann festgestellt werden, dass ES problemlos im Jugendstrafvollzug durchführbar ist. Im Hinblick auf die Besonderheiten einer offenen Anstalt, wie die spezifischen Alltagsabläufe aber auch die gewöhnlich recht kurzen Haftstrafen und die somit hohe Fluktuation der Inhaftierten, erscheint ES als kurze und unkompliziert zu implementierende Intervention geeignet. ES erwies sich als ein niedrig-schwelliges Angebot für jugendliche Inhaftierte, die auf 
Anfrage zumeist sofort bereit und interessiert waren, am Projekt teilzunehmen. Die Schreibintervention wurde demnach von fast allen angesprochenen Inhaftierten akzeptiert. Ihre Teilnahme bewerteten sie im nachhinein als positiv. Es sollte allerdings berücksichtigt werden, dass Prozesse der sozialen Erwünschtheit im Kontext von Untersuchungen in Strafanstalten deutlicher auf Ergebnisse Einfluss nehmen als dies ohnehin bei der Selbsteinschätzung mittels Fragebögen zu erwarten ist (Greve et al., 1996).

\subsubsection{Kurzfristige Effekte der Schreibintervention}

Entsprechend der Hypothese ergibt die varianzanalytische Überprüfung im Rahmen dieser Studie signifikante Gruppenunterschiede hinsichtlich der kurzfristigen emotionalen Reaktionen. Die paarweisen Vergleiche zwischen den Gruppen ergeben für die Experimentalgruppen (BE und TA) eine Zunahme an negativen und eine Abnahme an positiven Affekt. Dieser Befund entspricht zumindest soweit der ursprünglichen Annahme J.W. Pennebakers, als dass die Offenbarung persönlich belastender Erlebnisse für viele Menschen kurzfristig unangenehm ist. Die Vermutung liegt nahe, dass diese Annahme sich auch auf Offenbarungen bezüglich vergangener Straftaten ausweiten lässt und es sich hierbei ebenfalls um „belastende“ bzw. unangenehme Gefühle bereitende Erlebnisse handelt. Die Tatsache inhaftiert zu sein, führt darüber hinaus zu einer gewissen, wenn auch impliziten, Konfrontation mit der kriminellen Vergangenheit. Dass eine explizite Auseinandersetzung mit dieser Vergangenheit die Stimmung (zusätzlich) verschlechtert, zeigt sich im Vergleich zur Kontrollgruppe. Hier verbessert sich die Stimmung der Teilnehmer nach dem Schreiben, was sich eventuell damit erklären lässt, dass das Schreiben eine Ablenkung vom Vollzugsalltag darstellt und die Instruktion, den Alltag auf rein deskriptive Weise zu beschreiben, eine gut zu bewältigende Aufgabe ist.

Die kurzfristige Verschlechterung der Stimmung nach einer Schreibsitzung kann als ein verlässlicher Befund im Rahmen von ES-Studien betrachtet werden, da er in vielen Untersuchungen repliziert wurde (Horn, 2004; Kröner-Herwig et al., 2004; zusammenfassend Sloan \& Marx, 2004). Die Stimmungsverschlechterung wird dabei in erster Linie als Folge einer besonders intensiven Auseinandersetzung mit der belastenden Erfahrung interpretiert. Pennebaker (1993) geht davon aus, dass die Beschäftigung mit lang unterdrückten belastenden Emotionen und Gedanken naturgemäß zu einer kurzfristigen Stimmungsverschlechterung führt und nimmt an, diese sei notwendig für eine vollständige Verarbeitung des Erlebten und Voraussetzung für die gesundheitsförderlichen Effekte des ES. Diese Annahme konnte bisher empirisch nicht nachgewiesen werden (Smyth, 1998). 
Auch im Rahmen dieser Untersuchung konnte die aus der Theorie ableitbare Annahme, der kurzfristige Affekt stehe in Zusammenhang mit langfristig förderlichen Auswirkungen des ES, nicht bestätigt werden. Es finden sich keine Hinweise dafür, dass die kurzfristige Stimmungsverschlechterung eine mehr oder minder notwendige Voraussetzung für eine vollständige und erfolgreiche Verarbeitung des Erlebten und einem langfristig verbesserten Gesundheitsstatus darstellt. Auch Smyth (1998) konnte im Rahmen seiner Metaanalyse nicht nachweisen, dass kurzfristige Reaktionen auf das Schreiben in Zusammenhang mit langfristigen Verbesserungen des Wohlbefindens stehen. Eine explorative Überprüfung möglicher Zusammenhänge kurzfristiger und langfristiger Ergebnisparameter in der hier vorliegenden Studie zeigt gegenteilig, dass eine bessere Stimmung nach dem Schreiben mit einer langfristig stärkeren Abnahme der Belastungsreaktionen Intrusionen und Hyperarousal einhergeht. Hohe Werte Negativen Affekts korrelieren darüber hinaus mit einer langfristigen Zunahme an Verantwortungsablehnung, was bedeutet, dass eine kurzfristig schlechte Stimmung mit einer verstärkt antisozialen Haltung einher geht. Diese Ergebnisse beziehen sich auf die Experimentalgruppe, deren Teilnehmer eine schlechtere Stimmung direkt nach dem Schreiben aufweisen als die Teilnehmer der Kontrollgruppe. Ein übermäßiges Ausmaß an schlechter Stimmung direkt nach dem Schreiben erweist sich in dieser Studie als weniger langfristig „förderlich“.

\subsubsection{Längerfristige Wirkung auf körperliche und psychische Gesundheit}

In Bezug auf den subjektiven Gesundheitszustand zeigen sich keinerlei Effekte des ES. Dies gilt für die verschiedenen Maße wie Anzahl der Krankheitstage, der Arztbesuche, subjektive Angaben zu körperlichen Beschwerden oder der psychischen Symptombelastung. Hier blieben die erwarteten Verbesserungen in beiden Experimentalgruppen aus. Die Hypothese konnte somit nicht bestätigt werden.

Es zeigt sich jedoch eine signifikante Abnahme der Belastungsreaktionen, Intrusionen, Hyperarousal und Vermeidung, in den beiden Experimentalgruppen vom Zeitpunkt der Ersterhebung zur Folgeuntersuchung sechs Wochen nach der Intervention (partielles Eta ${ }^{2}=.34$ ). Symptome, die mit typischen psychischen Reaktionen auf Extremereignisse einhergehen, sind im prä-post-Vergleich also deutlich reduziert. Inwieweit dieser Befund jedoch der Wirkung des ES zuzuschreiben ist, bleibt weitgehend unklar, weil ein Vergleich mit der Kontrollgruppe nicht möglich ist, da diese den betreffenden Fragebogen aus bereits erläuterten Gründen nicht bearbeitet hat. Möglicherweise ist diese Abnahme an Belastungsreaktionen im Rahmen einer allgemeinen Symptomreduktion im Verlauf der Haft zu interpretieren. So zeigt sich ebenfalls 
eine signifikante Abnahme an psychischer Belastung, an Negativem Affekt und an Depressivität aller Teilnehmer im Verlauf der Zeit, die mit einer allgemeinen Anpassung an die Haftbedingungen und einer generellen emotionalen Stabilisierung der Jugendlichen erklärt werden kann. Es konnte gezeigt werden, dass sich nach einer Akklimatisierungsphase von drei bis vier Monaten der psychische Zustand der meisten jugendlichen Inhaftierten auf ein normales Niveau stabilisiert (Hosser, 2004). Andererseits handelt es sich bei den Fragen bezüglich der Belastungsreaktionen um Angaben zu einem spezifischen Ereignis, nämlich dem, über das der Teilnehmer geschrieben hat. Es wurde demnach kein genereller Befindlichkeitszustand abgefragt, sondern die Teilnehmer haben Angaben zu Symptomen von Hyperarousal, Intrusionen und Vermeidung bezüglich eines spezifischen Ereignisses gegeben. Üblicherweise verändern sich diese durch Gewöhnungs- oder Anpassungsprozesse an veränderte Lebensumstände. Dennoch kann auch im Rahmen dieser Untersuchung nicht ausgeschlossen werden, dass der Befund auf einen nicht unüblichen spontanen Rückgang der Belastungssymptome zurückzuführen ist, der sich ohne jegliche Form der Behandlung einstellen kann (Ehlers, 2000).

Die ohnehin gute gesundheitliche Verfassung der Teilnehmer zu Beginn der Studie und ein damit verbundener Bodeneffekt könnte eine mögliche Erklärung für das Ausbleiben gesundheitsförderlicher Auswirkungen des ES sein. Dieser schlägt sich beispielsweise in den nahe Null liegenden Ausgangswerten für die Anzahl von Arztbesuchen und Krankheitstagen nieder. Eine erwartete signifikante Verbesserung des subjektiven Gesundheitszustandes in den Experimentalgruppen hätte demnach nur zustande kommen können, wenn die Teilnehmer zum Follow-up Zeitpunkt fast völlig beschwerdefrei gewesen wären. Im Rahmen dieser Studie fällt zudem auf, dass die Teil- nehmer der Gruppen Belastendes Erlebnis und die der Kontrollgruppe im prä-post-Vergleich eine erhöhte Anzahl an Krankheitstagen und Arztbesuchen aufweisen, was zusätzlich gegen die Erwartung eines gewünschten Effektes bezogen auf die Teilnehmer der Gruppe Belastendes Erlebnis spricht und unter anderem im Haftkontext damit erklärt werden könnte, dass durch eine Krankmeldung wenig beliebte Arbeitsaufgaben umgangen werden können. Andererseits zeigten sich in früheren Studien mit Probanden, die ebenfalls in einem guten Gesundheitszustand waren, gesundheitsförderliche Effekte des ES (z.B. Pennebaker \& Beall, 1986; Pennebaker et al., 1990). In Studien, in denen die Anzahl der Arztbesuche als Maß für den gesundheitlichen Status eines Probanden herangezogen worden ist, und sich diese Anzahl im Vergleich zur Kontrollgruppe nach der Teilnahme an der Schreibintervention vermindert hat, fällt auf, dass häufig gefundene Gruppenunterschiede auf einer Zunahme der Arztbesuche in der Kontrollgruppe im Vergleich zur gleich bleibenden Häufigkeit in der Experimentalgruppe beruht (Greenberg et al., 1996; Pennebaker \& Beall, 
1986; Pennebaker \& Francis, 1996). So kommen auch Meads und Nouwen (2005) im Rahmen ihrer Metaanalyse auf der Grundlage 61 berücksichtigter Primärstudien bezüglich des Maßes der objektiv erfassten Anzahl an Arztbesuchen (Health Care Utilization) zu dem Ergebnis, dass sich im Vergleich zwischen Experimental- und Kontrollgruppe langfristig keine signifikanten Unterschiede ermitteln lassen.

Eine ähnliche metaanalytische Befundlage ergibt sich für die Maße psychischer Gesundheit (Frattarolli, 2006; Frisina et al., 2004; Mogk et al., 2006). Im Rahmen der hier vorliegenden Studie konnte keine längerfristige Verbesserung der Affektivität durch die Intervention nachgewiesen werden. Überdies ergaben sich weder signifikante Verbesserungen bezüglich der Ergebnismaße für Zustandsangst noch bezüglich der Werte für Depressivität in den Experimentalgruppen. Lediglich in Bezug auf das Maß Zustandsärger ergab sich ein signifikanter Interaktionseffekt dergestalt, dass die Teilnehmer der Gruppe Belastendes Erlebnis eine tendenzielle Abnahme zeigen, wohingegen die Teilnehmer der Gruppe Tataufarbeitung eine Zunahme an Ärger zum Follow-up Zeitpunkt aufweisen. Aufgrund einiger statistischer Einschränkungen ist dieses Ergebnis jedoch mit Vorsicht zu interpretieren. In anschließenden Einzeltestungen ergaben sich keine signifikanten Gruppenunterschiede hinsichtlich der präpost-Differenzen. Der Interaktionseffekt könnte in diesem Zusammenhang bedeuten, dass die Teilnehmer der Gruppe Tataufarbeitung zum Zeitpunkt der Folgeerhebung sich an ihr Schreibthema (vergangene Straftat) erinnern und sie dies im Gegensatz zur Gruppe Belastendes Erlebnis eher frustriert und ärgerlich sein lässt. Durch ihre Teilnahme am Projekt und ihre Zugehörigkeit zur Gruppe Tataufarbeitung werden sie auf diese Weise erneut mit ihren Straftaten konfrontiert. Der Umstand der andauernden Inhaftierung könnte ihnen vermehrt vor Augen geführt haben, dass die beschriebenen Ereignisse letztlich zu ihrem Freiheitsentzug geführt haben, was widerum Ärger auslösen könnte.

\subsubsection{Längerfristige Wirkung auf Maße der Normorientierung und Führung}

Die Hypothese, Expressives Schreiben über vergangene Straftaten wirke sich langfristig positiv auf die Normorientierung, die Verantwortungsübernahme und die Führung während der Haftzeit aus, konnte nicht bestätigt werden. Die Teilnehmer der Gruppe Tataufarbeitung zeigten demnach keine Veränderung in den Selbstauskunftsmaßen bezüglich der Normorientierung oder in ihrem Führungsverhalten im Vergleich zur Gruppe Belastendes Erlebnis und der Kontrollgruppe. 
Es konnte gezeigt werden, dass sich ein Aspekt des moralischen Urteils, nämlich die Einschätzung, dass es sich lohnt, Straftaten zu begehen, im Verlauf der Zeit bei den Teilnehmern aller drei Gruppen abnimmt. Hierbei könnte es sich schlicht und einfach um einen - durchaus erwünschten - Effekt der Inhaftierung und Strafverbüßung handeln, wobei sich die individuelle Bewertung dahingehend verändert, dass ein möglicher durch die Straftat erzielter „Gewinn“ die Konsequenzen des Freiheitsentzuges nicht länger überwiegt.

Ein überraschendes Ergebnis zeigt sich schließlich bezüglich der Führungsmaße. Die durchschnittliche Führungsbenotung fällt für die Teilnehmer der Gruppe Belastendes Erlebnis signifikant besser aus als für die Gruppe Tataufarbeitung. Die Benotungen der Gruppe Belastendes Erlebnis erweist sich im Vergleich zur Kontrollgruppe als tendenziell besser, erreicht im Rahmen der post-hoc-Testungen aber keine statistische Signifikanz. Bezüglich der Anzahl der Anhörungen, die den Gefangenenakten entnommen wurden, zeigen die Teilnehmer der Gruppe Belastendes Erlebnis signifikant weniger Anhörungen als die Teilnehmer der beiden anderen Gruppen. Im Rahmen der Interpretation dieses Ergebnisses ist einschränkend zu berücksichtigen, dass die Führungsmaße nicht im prä-post-Vergleich betrachtet werden konnten, sondern ausschließlich Durchschnittwerte bezogen auf einen viermonatigen Follow-up Zeitraum verglichen wurden, wonach nicht restlos auszuschließen ist, dass sich zufällig gut führende Inhaftierte in der Gruppe Belastendes Erlebnis befunden haben, die unabhängig von ihrer Teilnahme am Projekt besser benotet worden sind. Durch die randomisierte Zuteilung der Teilnehmer zu den Gruppen wurde allerdings versucht, einem solchen Effekt entgegenzuwirken. Eine Überprüfung dieses Einflusses mittels prä-Werte-Vergleich war nicht möglich, da die Teilnehmer aufgrund zumeist kurzer Haftstrafen im Jugendstrafvollzug zu Beginn ihrer Haftzeit für die Studie rekrutiert wurden, zu einem Zeitpunkt, an dem gewöhnlich noch keine Führungsbenotung durch die Mitarbeiter der Anstalt vorgenommen wird. Für die Güte der Daten spricht andererseits, dass diese nicht auf der Selbstauskunft der Teilnehmer beruhen und somit Effekte der sozialen Erwünschtheit keine Rolle spielen. Es ist davon auszugehen, dass die unterschiedlichen Mitarbeiter der JVA, die die Benotungen der Bereiche Schule/Arbeit, Sport/Freizeit und Verhalten auf der Wohngruppe abgeben, der Intervention gegenüber vollständig „blind“ gewesen sind. Da es sich um verhaltensnahe Maße handelt, spiegeln sie das tatsächliche oder vielmehr beobachtete alltägliche Verhalten der Teilnehmer vor Ort in den verschiedenen Wohn- und Lebensbereichen wider. Auch hier sind Bemühungen, sich möglichst regelkonform zu verhalten, zu erwarten und werden sogar institutionell verstärkt. So handelt es sich bei Regelverstößen, die zu einer schlechteren Benotung führen, beispielsweise um unerlaubten Handybesitz, Alkohol- und Drogenkonsum, Streitereien mit Mitgefan- 
genen oder dem Personal der Anstalt. Ein Rückschluss auf das Wohlverhalten nach der Haftentlassung ist somit nicht möglich. Regelkonformes Verhalten während der Haft z.B. im Sinne einer kooperativen Haltung kann allerdings durchaus als ein Indikator für eine positive Prognose gewertet werden und ist daher zugleich von legalprognostischer Bedeutung (Endres, 2004).

Es stellt sich nun die Frage, warum das Schreiben über ein belastendes Lebensereignis auf emotional expressive Weise dazu geführt hat, dass sich die Inhaftierten in der Folgezeit besser führten als solche, die über die eigenen Straftaten oder ihren Tagesablauf schrieben. Folgt man den theoretischen Annahmen emotionsregulatorischer Wirkmechanismen des ES, könnte argumentiert werden, dass es durch das ES zu einer verbesserten Emotionsregulation und somit zu einer besseren Adaptation an die verschiedenen Lebensbereiche im Haftalltag gekommen ist. Es könnte angenommen werden, dass die Jugendlichen nach der Reflektion belastender Erlebnisse diese in der Folge besser verarbeitet haben, was unter Umständen zu einer erhöhten Impulskontrolle geführt haben kann. Sich selbst als emotional ausgeglichener und eigenes Verhalten als kontrollierter zu erleben, hat direkt Auswirkungen auf das soziale Umfeld und wirkt längerfristig verstärkend. Erklärungsmodelle des ES, die auf soziale Faktoren abzielen, postulieren, dass das Formen eines kohärenten Narrativs belastende Erfahrungen besser kommunizierbar macht und es ermöglicht, in veränderter Weise auf das soziale Umfeld zuzugehen (Horn, 2004). Es konnte gezeigt werden, dass die gezielte Reflektion autobiografischer Erlebnisse zu einer verbesserten sozialen Problemlösefähigkeit führen kann (Goddard, Dritschel \& Burton, 1996). Im Gegensatz dazu zeigen vermeidende Coping- und Emotionsregulationsstiele hohe korrelative Zusammenhänge mit Werten niedriger sozialer Kompetenzen zeigen (Eisenberg, Cumberland \& Spinrad, 1998). Eine gedanklich-emotionale Integration von belastenden Erlebnissen könnte eine Unterbrechung von einem vermeidendem Umgang mit emotional besetzten Gedächtnisinhalten darstellen und eventuell den Zugriff auf solche Gedächtniskomponenten ermöglichen, die für den Umgang mit sozialen Situationen relevant sind. Die adäquate Messung abhängiger Variablen, die mögliche zu erwartende subtile (soziale) Effekte abbilden, bleibt in zahlreichen Forschungsbemühungen bezüglich ES allerdings eine methodische Herausforderung (Horn, 2004; Stone, Smyth, Kaell \& Hurewitz, 2000). Untersuchungen mittels Mikroaudiorekorder nach der Teilnahme am ES sind daher bemüht, alltägliche non-reaktive Verhaltensspuren zu erfassen und mittels Verhaltenskodierung und linguistischer Analysen hinsichtlich sozialer und sprachlicher Aspekte auszuwerten (Mehl, Pennebaker, Crow, Dabbs \& Price, 2001). Hierbei konnte gezeigt werden, dass die Probanden der Experimentalgruppe im Vergleich zur Kontrollgruppe und zur Baseline nach dem Schrei- 
ben mehr sprachen, mehr soziale Wörter, mehr positive Emotionswörter und weniger Ärgerwörter benutzten (Mehl \& Pennebaker, 2000). Da dieser Effekt für Männer deutlich stärker ausfiel, liegt die Vermutung nahe, dass auch im Rahmen dieser Studie soziale und linguistische Prozesse als mögliche Wirkfaktoren eine Rolle gespielt haben könnten. Überprüfbar wäre diese Annahme selbstverständlich lediglich im Rahmen einer Studie im Strafvollzug, die nach einer Schreibintervention ebenfalls alle Gespräche der Teilnehmer aufzeichnet.

Neben diesen theoretischen Überlegungen spricht jedoch gegen eine Überbewertung der Befunde bezüglich der Führungsnoten der in allen Gruppen vorliegende, relativ niedrige mittlere Notendurchschnitt, der sich um die Note ,3“ bewegt. Bei der Praxis der Benotung sind extreme Bewertungen wie ein „sehr gut“ oder ein „mangelhaft“ praktisch nicht vorgekommen. Statistisch betrachtet liegt der Anteil der Gesamtvariabilität, der dem Faktor Gruppe zugeschrieben werden kann, mit einer Effektstärke von $\mathrm{Eta}^{2}=.12 \mathrm{im}$ unteren Bereich und ist daher als gering zu bewerten.

Interessanterweise zeigt sich im Rahmen der explorativen Untersuchung, dass Teilnehmer der Experimentalgruppen, die lange Haftstrafen zu verbüßen haben, bessere Führungsnoten haben als kurzstrafige Probanden und zudem eine Zunahme an Normorientierung zeigen. Dies kann zusätzlich bedeuten, dass eine längere zeitliche Perspektive in der Haft die Motivation für regelkonformes Verhalten erhöht.

\subsubsection{Moderatoreffekte}

Bevor auf die Interpretation der Ergebnisse bezüglich der verschiedenen Moderatorvariablen näher eingegangen wird, soll darauf hingewiesen werden, dass mögliche Effekte von Moderatoren nur schwer aufdeckbar sind, wenn - wie in dieser Studie vorgefunden - die Wirkung des ES sehr gering ist. Unter dieser Voraussetzung ist die Interpretation eingeschränkt.

Für das Merkmal Alexithymie ließ sich kein Zusammenhang mit der überwiegenden Mehrzahl der Ergebnismaße aufzeigen und die Annahme, das Ausmaß an Alexithymie zeige einen moderierenden Einfluss hinsichtlich der Wirkung des ES auf verschiedene abhängige Variablen, konnte somit im Rahmen dieser Untersuchung nicht bestätigt werden. Ein Einfluss dieses Merkmals hat in verschiedenen Schreibstudien z.T. konträre Ergebnisse erbracht. In vielen Untersuchungen konnte hingegen keinerlei moderierende Wirkung nachgewiesen werden (z.B. Kröner-Herwig, 2004; Lumley, 2004). Im Rahmen verschiedener Studien wurde zunächst erwartet, ein hohes Ausmaß an Alexithymie und eine damit einhergehende Beeinträchtigung in der Fähigkeit, eigene Gefühle wahrzunehmen und auszudrücken, vermindere den 
positiven gesundheitlichen Effekt des ES (zusammenfassend bei Lumley, 2002). Zur Überraschung einiger Autoren ergab sich jedoch ein gegenteiliger Effekt und es konnte gezeigt werden, dass hoch-alexithyme Probanden anscheinend in stärkerem Maße vom ES profitierten als solche, die einen mittleren oder niedrigen Alexithymie-Wert erzielten (Paez et al., 1999; Solano et al., 2003). Paez et al. (1995) berichteten Ergebnisse, wonach sich ein Moderatoreffekt des Merkmals Alexithymie insofern bestätigte, als hoch-alexithyme Probanden eine ausgeprägtere Reduktion ihres Vermeidungsverhaltens sowie eine erhöhte Verbesserung der Affektivität aufwiesen als Probanden mit niedriger Alexithymieausprägung. In Bezug auf das Ausmaß an Vermeidung ergeben sich im Rahmen dieser Untersuchung ähnliche Befunde wie bei Paez und Mitarbeitern. Bei hoch-alexithymen Teilnehmern nimmt das Ausmaß an Vermeidung bezüglich des belastenden Erlebnisses bzw. der Straftat ab. Eine Verbesserung der Affektivität ließ sich nicht bestätigen. Teilnehmer mit niedrigen Alexithymie-Werten zeigten hingegen eine stärkere Abnahme an Ärger und negativem Affekt.

Diese unterschiedlichen und teilweise widersprüchlichen Ergebnisse bezüglich des Merkmals Alexithymie im Rahmen von Schreibstudien sind vermutlich auf die Heterogenität des Konstrukts Alexithymie und die damit verbundenen Schwierigkeiten bei der Entwicklung eines reliablen und validen Messinstruments zurückzuführen. Die TAS (-20) stellt zwar einerseits ein reliables Messintrument zur Erhebung von Alexithymie dar, weist andererseits aber eine recht geringe interne Konsistenz auf. So konnte gezeigt werden, dass lediglich erhöhte Werte auf den Subskalen Schwierigkeiten eigene Gefühle zu beschreiben und Schwierigkeiten die eigenen Gefühle zu identifizieren im Rahmen von Schreibstudien moderierende Wirkung aufweisen (Lumley, 2004). Alexithymie scheint weniger ein theoretisch geschlossenes Konzept zu sein als vielmehr aus einigen theoretisch wie methodisch von einander abgrenzbaren Komponenten zu bestehen. Daher überrascht es nicht, dass sich auch metaanalytisch keine signifikante Moderatorwirkung von Alexithymie beim ES nachweisen lässt (Frattarolli, 2006). Selbstverständlich gilt es zu berücksichtigen, dass es sich fast ausschließlich um korrelative Analysen potentieller Moderatoren handelt und Ursache-Wirkung-Zuschreibungen nicht möglich sind. Aufgrund der fehlenden Geschlossenheit des theoretischen Konzepts ist es vorstellbar, dass Einflüsse, die in manchen Schreibstudien dem Konstrukt Alexithymie zugeschrieben wurden, auch in Zusammenhang mit anderen Persönlichkeitsvariablen (z.B. Ängstlichkeit, Offenheit) stehen, die ihrerseits mit Alexithymie korrelieren.

Das Vorhandensein der Diagnose einer Antisozialen Persönlichkeitsstörung wies im Rahmen dieser Studie keinen Zusammenhang mit der überwiegenden Mehrheit der Ergebnismaße 
auf. Lediglich in der Einschätzung, ob es sich lohnt, Straftaten zu begehen, ergibt sich ein Einfluss des Vorhandenseins einer APS-Diagnose derart, dass diese Überzeugung abnimmt, wohingegen sie bei Probanden ohne APS-Diagnose zunimmt. Dieses Ergebnis kann nur eingeschränkt interpretiert werden, da sich sowohl statistisch wie inhaltlich einige Probleme ergeben. Zum einen ist die statistische Voraussetzung der Gleichheit der Kovarianzmatrizen der abhängigen Variablen für die multivariate Berechnung möglicher Unterschiede verletzt. Zum anderen ergibt sich der beobachtete Unterschied nicht auf der anderen Subskala der umgekehrten Einschätzung, nämlich, dass es sich nicht lohnt, Straftaten zu begehen. Hierbei spielen möglicherweise im Antwortverhalten der Probanden Effekte sozialer Erwünschtheit eine erhebliche Rolle, so dass inhaltlich gleiche, aber formal umgepolte Items in ihrer Aussage unterschiedlich beurteilt werden.

Hinsichtlich der Belastungsreaktionen Intrusionen und Hyperarousal zeigen sich moderierende Einflüsse des Vorhandenseins bzw. Fehlens der Diagnose. Entgegen der Erwartung verbessern sich diese Symptome bei solchen Teilnehmern, die eine APS-Diagnose erhielten. Die Abnahme von Symptomen wie etwa Durchschlafschwierigkeiten, Reizbarkeit oder Hellhörigkeit sollten im Rahmen der Interpretation erneut im Zusammenhang mit einem Gewöhnungsprozess an die Haftsituation gesehen werden. Gerade bei Teilnehmern mit Störungen des Sozialverhaltens ist es denkbar, dass diese vielgestaltigen Beeinträchtigungen in Form von Belastungsreaktionen zu Beginn einer Inhaftierung stärker ausgeprägt sind bzw. vom Betroffenen eine Abnahme an Symptomen verstärkt wahrgenommen wird. Es ist nicht auszuschließen, dass Inhaftierte, die die Kriterien einer APS erfüllen, vermehrt belastende Lebensereignisse erlebt haben und oft auch in mehr kriminelle Aktivitäten involviert gewesen sind. Dennoch ist nicht auszuschließen, dass ES bei diesen Teilnehmern tatsächlich zu einer Symptomreduktion geführt haben könnte. Wobei fraglich bleibt, ob es die diagnostischen Kriterien wie beispielsweise das überdauernde Muster von Missachtung und Verletzung der Rechte anderer sind, die spezifisch über ES positiv beeinflusst wurden oder spezifische der Störung zugehörige Merkmale wie etwa niedriger sozioökonomischer Status, vielgestaltige Probleme in der Herkunftsfamilie, Heimaufenthalte oder andere psychische Probleme wie etwa Aufmerksamkeitsdefizit-/Hyperaktivitätsstörungen, die diese Gruppe kennzeichnen und sie daher eher vom Schreiben profitieren lassen.

In einigen Studien konnte gezeigt werden, dass manche Essaymerkmale wie die häufige Verwendung bestimmter Wortkategorien, wie beispielsweise positive bzw. negative Emotionsworte oder Kausalitätsworte langfristig förderliche Auswirkungen von ES verstärken (Horn, 
2004). Diese Annahme konnte im Rahmen der hier vorliegenden Studie lediglich für zwei Ergebnismaße bestätigt werden. Eine häufige Verwendung positiver Emotionsausdrücke korreliert mit einer besseren Führungsbenotung. Der häufige Gebrauch positiver Emotionsausdrücke könnte die Folge einer besonders erfolgreichen Assimilation und Neubewertung eines belastenden Erlebnisses darstellen und somit ein relevanter Wirkmechanismus der Selbstöffnung darstellen (Linkemann, 2001). Eine sich im häufigen Gebrauch positiver Emotionsworte ausdrückende versöhnliche Einstellung gegenüber der eigenen belastenden Vergangenheit könnte sich hilfreich auf das eigene Denken und Handeln auswirken. Folgt man diesem Gedankengang, dann bildet die Häufigkeit positiver Emotionsworte im Sinne einer Prozessvariablen eine förderliche Wirkung des ES ab, die sich letztlich auf der Verhaltensebene zeigt. Zur Einschätzung der Richtigkeit dieses Gedankens fehlen allerdings noch die Überprüfung einiger Teilschritte dieses Wirkmechanismus sowie Befunde von weiteren Schreibstudien, die sowohl Verhaltensdaten erheben wie auch Inhaltsanalysen durchführen. Unklar bleibt überdies, inwieweit eine häufige Verwendung positiver Emotionsworte auch Ausdruck eines grundlegenden Wohlbefindens, einer guten Stimmung, einer optimistischen Einstellung oder anderer personenbezogenen Variablen sein kann und nicht notwendigerweise zusätzlichen Aufschluss über differenzierte Wirkweisen des ES auf der rein linguistischen Ebene gibt. Eine ähnliche Interpretation ist auch im Fall der Häufigkeit von Kognitionsworten in Betracht zu ziehen. Eine gesteigerte Verantwortungsübernahme für die eigene Straffälligkeit korreliert hier mit einem erhöhten Anteil von Kognitions- und Einsichtsworten. Wobei es einleuchtend ist, dass die Fähigkeit und Bereitschaft zur Reflektion der persönlichen Vergangenheit in Zusammenhang mit einer sich während der Haftzeit entwickelnden Verantwortungsübernahme steht. Dieser Effekt ist mit Sicherheit wünschenswert und sollte bei der Planung ähnlicher Forschungsarbeiten im Strafvollzug berücksichtigt werden. Gerade im Hinblick auf die Auseinandersetzung mit dem kriminellen Verhalten ist durch ES eine häufig zu beobachtende Vermeidung persönlich unangenehmer Themen nicht länger möglich, ganz gleich, ob die Fähigkeit zur Reflektion persönlicher Erlebnisse zuvor bereits bestanden hat oder nicht. 


\subsection{Schlussfolgerung und Ausblick}

\subsubsection{Expressives Schreiben als sinnvolle Methode in der Arbeit mit jugendlichen Inhaftierten?}

Die hier vorgestellte Studie weicht hinsichtlich vieler Faktoren von anderen Schreibstudien und vom klassischen Schreibparadigma Pennebakers ab. Für eine abschließende Beurteilung der Ergebnisse und eines möglichen Vergleichs mit anderen Studien sei darauf hingewiesen, dass es sich bei den Teilnehmern, inhaftierten Jugendlichen, um eine bisher einmalige Klientel im Rahmen der Anwendung von ES handelt. Neben den verschiedenen Eigenschaften und Merkmalen dieser Gruppe, spielt sicherlich auch der Inhaftierungskontext für die Bewertung der Ergebnisse dieser Studie eine Rolle. Auch bei durch externe Forscher durchgeführten Untersuchungen sind Effekte sozialer Erwünschtheit im Inhaftierungskontext in vergleichbar hohem Maße zu erwarten. Der Befund, dass alle Teilnehmer, einschließlich die der Kontrollgruppe, ihrer Teilnahme am Projekt positiven Nutzen abgewinnen konnten, macht diesen Effekt einmal mehr deutlich. In gewöhnlichen Schreibstudien, die überwiegend mit studentischen Probanden durchgeführt wurden, ist eine solche Bewertung durch die Kontrollgruppe wenig wahrscheinlich. In einer Schreibstudie mit deutschen Studenten und Studentinnen wurden mögliche positive Auswirkungen des Schreibens von den Teilnehmern der Experimentalgruppe subjektiv als signifikant höher eingeschätzt als von den Teilnehmern der Kontrollgruppe (Linkemann, 2001).

Es kann überdies angenommen werden, dass ein gewisses Misstrauen, dass Informationen, die aus der Studie gewonnen werden, letztlich doch zu den Mitarbeitern und Verantwortlichen der Anstalt gelangen könnten, bei den meisten jugendlichen Teilnehmern bestanden hat. Dies ist bei der Planung und Durchführung von Interventionsmaßnahmen im Haftkontext besonders zu beachten. Um so bemerkenswerter erscheint jedoch die Tatsache, dass sich viele angesprochene Inhaftierte zu einer Mitarbeit bereit zeigten und darüber hinaus der Instruktion, über sehr persönliche Erlebnisse auf emotional expressive Weise zu schreiben, folgten.

Bei der Durchsicht der Essays bestätigte sich allerdings auch die Vermutung, dass Sprachund Schreibkenntnisse vieler Teilnehmer als recht gering einzuschätzen sind. Die Fähigkeit, sich in Art eines Tagebuchschreibens mit den eigenen Gedanken zu beschäftigen und schriftlich auszudrücken, ist für viele von ihnen eine wenig vertraute Aufgabe - nur ein Teilnehmer gab an, überhaupt jemals ein Tagebuch geführt zu haben - und stellt somit gerade für die Teilnehmer der Experimentalgruppen eine nicht zu unterschätzende Herausforderung dar. In diesem Zusammenhang bleibt allerdings unklar, inwieweit Schreibtüchtigkeit und regelmäßi- 
ge Selbstreflexion eine notwendige Voraussetzung für einen möglichen (gesundheits-) förderlichen Effekt des ES darstellen. Schreibstudien im Sinne Pennebakers mit (jugendlichen) sozial eher randständigen Probanden mit geringem Bildungsniveau bleiben bisher eine Ausnahme (Evans, 2000; Richards, 2000; Tromp, 1998) und bieten somit wenig Basis für einen Vergleich von Durchführungsbedingungen und Ergebnissen. Bei den überwiegend mit studentischen Populationen durchgeführten Studien dürfte das durchschnittliche Schreib- und Ausdrucksvermögen der hier untersuchten Gruppe überlegen sowie das Bildungsniveau um einiges höher gewesen sein (vgl. Frattarolli, 2006).

Eine weitere wesentliche Abweichung dieser Studie im Vergleich zu den meisten anderen Schreibstudien, in denen über ein belastendes oder traumatisches Ereignis geschrieben wurde, besteht in der zusätzlichen Instruktion, über die vergangene Straftat(-en) zu schreiben. Diese Instruktion ist aber wiederum in Anlehnung an Schreibthemen, die eine schriftliche Auseinandersetzung mit Themen wie Krankheit, Beziehungserfahrungen, Scham oder Schuld beinhalten, zu sehen. Diese können ebenfalls nicht einfach der Kategorie „,belastendes Erlebnis“ zugeordnet werden und unterscheiden sich von daher vom Ursprungsparadigma. Das Schreiben über so verschiedene Themen kann eine Vielzahl emotionaler und kognitiver Aspekte wirksam werden lassen und macht die Anwendung spezieller theoretischer Annahmen im Rahmen der Interpretation möglicher Wirkmechanismen schwierig. In dieser Untersuchung zeigte sich, dass sich die schriftliche Auseinandersetzung mit der vergangenen Straftat im Vergleich zum Schreiben über das belastendste bzw. „schlimmste“ Lebensereignis bei jugendlichen Inhaftierten nicht positiv auf das Verhalten während der Haft auswirkt. Es kann vermutet werden, dass ein alleiniges Sich-vor-Augen-Führen von Aspekten der kriminellen Vergangenheit nicht zu einer nachhaltigen Veränderung antisozialer Einstellungen oder normkonformen Verhaltens führt und als Kurzintervention in einer Haftanstalt der Modifikation bedarf. Möglicherweise wirken Inhaftierung und Konfrontation mit einer Straftat, die eventuell in unmittelbarem ursächlichen Zusammenhang mit der Inhaftierung steht, sogar ungünstig und lösen wenig konstruktive Schuldgefühle aus, die das Kompetenzerleben des Jugendlichen nicht zu verbessern vermögen. Dagegen könnte eine gezielte Auseinandersetzung mit den oft zahlreichen individuellen Entstehungsbedingungen von wiederholtem kriminellen Verhalten und eine längerfristige (sozial-) therapeutische Begleitung im Einzel- und Gruppensetting z. B. im Rahmen eines sozialen Trainingskurses eine konstruktive Form der Straftataufarbeitung möglich machen. 
Damit stellt sich die Frage, inwieweit ES überhaupt sinnvoll im Kontext einer Jugendstrafanstalt eingesetzt werden kann. Im Sinne einer isolierten Interventionsmaßnahme, in deren Rahmen ein jugendlicher Inhaftierter drei bis vier Mal über ein belastendes Lebensereignis schreibt, kann ES als wenig effektiv eingeschätzt werden. Die ursprünglich angenommene gesundheitsförderliche Wirkung des Schreibens kann trotz einer recht großen Anzahl an Schreibstudien bis heute nicht nachgewiesen werden. Mögliche andere Effekte des ES wie z.B. eine Verbesserung des Gesundheitsverhaltens scheinen, soweit sie gruppiert betrachtet worden sind, ebenfalls nicht nachweislich. Wie Frattarolli (2006) im Rahmen ihrer Metaanalyse betont, sollte es einen skeptisch machen, falls eine derart kurze Intervention ohne soziales Feedback eine ähnlich hohe Wirksamkeit besäße wie beispielsweise eine ambulante Psychotherapie. Dennoch bleibt offen, was ES denn nun eigentlich leisten kann und warum es überhaupt noch eingesetzt werden sollte. Denn trotz der neueren metaanalytischen Befunde wird ES weiterhin in verschiedenen Kontexten und bei unterschiedlichen Stichproben eingesetzt. So lassen sich aktuell Schreibstudien zu Themen wie beispielsweise das Schreiben über Lebensziele (Harrist, Carlozzi, McGovern \& Harrist, 2007), die Veränderung von Gedächtnisinhalten durch Selbstöffnung (Pasupathi, 2007), ES als Raucherentwöhnungsbehandlung bei Jugendlichen (Ames et al., 2007), internetbasiertes ES (Tatti, 2007) oder die Wirkung von ES auf die Qualität der Partnerbeziehung (Eells, 2007) finden - mit erwartungsgemäß uneinheitlichen Befunden bezüglich der Wirksamkeit vom ES. Ein Unterschied zu älteren Schreibstudien zeigt sich im augenscheinlich häufigeren Einsatz computerbasierter inhaltsanalytischer Auswertungen der Essays, mit deren Hilfe versucht wird, Aufschluss über die Mechanismen erfolgreichen Schreibens zu erhalten (z.B. Hughes, 2007; Pasupathi, 2007). In einer ebenfalls neuen Studie von Winslett (2007) konnte interessanterweise gezeigt werden, dass sich die Annahme, ES könnte bei jugendlichen Probanden die allgemeine Anpassung verbessern, ihre längerfristige Bereitschaft zur Selbstöffnung gegenüber Freunden fördern und somit die Intimität von engen Freundschaften zu Gleichaltrigen erhöhen, bestätigen ließ. Die Geschlechtszugehörigkeit hatte hierbei keinen Einfluss auf die Ergebnisse.

Im Rahmen des Strafvollzugs ist vorstellbar, ES zum einen in Form des Führens eines Tagebuchs einzusetzen - sofern ein Inhaftierter dies nicht bereits tut. Persönliche Probleme schriftlich darzulegen und sich auf diese Weise bestimmten Gedanken und Gefühle zu stellen, ist in den meisten Fällen zumindest nicht kontraproduktiv, vorausgesetzt, der Jugendliche hat im weiteren Umgang mit belastenden Themen einen Ansprechpartner. Zum anderen könnte es angezeigt sein, ES im Sinne einer zusätzlichen Maßnahme, wie etwa im Rahmen bestimmter 
gruppentherapeutischer Maßnahmen, wie beispielsweise sozialer Trainingskurse oder AntiAggressivitäts-Trainings als eine Vorbereitung und/oder Hausaufgabe in der Aufarbeitung verschiedener persönlicher Themen, wie z.B. der eigenen Kindheit oder Jugend eingesetzt werden. Eine schriftliche Vor- oder Nachbereitung, bei der sich emotional expressiv ausgedrückt werden soll, kann die Funktion haben, einen therapeutischen Einstieg in sehr persönliche Themen zu erleichtern. Sie könnte die Kompetenz erhöhen, solche persönlichen Informationen $\mathrm{zu}$ verbalisieren und eine Wiederholung zur Steigerung des Lerneffektes bereits besprochener Coping-Strategien außerhalb des therapeutischen Settings ermöglichen. In diesem Zusammenhang erscheint es erwähnenswert, dass neuerdings auch Aspekte der Selbstbestätigung in Bezug auf mögliche Wirkmechanismen des ES diskutiert werden (Creswell, Stanton, Taylor, Bower \& Sherman, 2007) woran sich die Vermutung einer Verstärkung des Selbstwirksamkeitsempfindens sinnvoll anknüpfen lässt.

Eine schriftliche Vorbereitung von straftatrelevanten Themen existiert - wenn auch auf etwas andere Weise als beim klassischen ES - in Form so genannter Opferbriefe, bei denen ein Gefangener die Aufgabe hat, an das Opfer einen Brief zu verfassen und diesen dann zumeist im Gruppensetting vorzustellen. Ziele dieser Maßnahme bestehen darin, für die Lage des Opfers Empathie zu entwickeln, Schuld und Reue auszudrücken und sich bei ihm zu entschuldigen. Einen derartigen „Opferbezug“ gerade bei schweren Straftaten herzustellen, wird als ein wesentliches Element der Förderung sozialer Verantwortung im Strafvollzug betrachtet werden (Rössner, 2005).

\subsubsection{Ausblick}

Die ausbleibende Wirkung des Expressiven Schreibens auf den subjektiven Gesundheitsstatus, das psychische Wohlbefinden sowie auf die Normorientierung steht im Wesentlichen im Einklang mit den Ergebnissen aktueller Metaanalysen.

Für die Planung weiterer Schreibstudien im Haftkontext, die in Form einer isolierten Maßnahme - wie bereits erläutert - wenig sinnvoll und effektiv erscheinen, ist es ratsam, im Hinblick auf eine ausreichend große Teststärke mit größeren Stichproben zu arbeiten, um mögliche Effekte aufzudecken. Weiterhin ist es empfehlenswert, verschiedene Verhaltensdaten (z.B. Maße der Führung oder des Sozial- und Arbeitsverhaltens) in die Analyse aufzunehmen. 
Diese Untersuchung hat nicht nur gezeigt, dass sich bei dieser Art von Daten Veränderungen zeigen können, sie stellen überdies Beobachtungen Dritter während der Haft und somit ein ökologisch valides Maß dar, das außerdem von prognostischer Bedeutung ist. Zugleich erscheint es ratsam, verschiedene mögliche auf die Ergebnismaße Einfluss nehmende Variablen (wie z.B. Straflänge) zu berücksichtigen. Weiterhin bietet eine inhaltsanalytische Auswertung der geschriebenen Essays die Möglichkeit, Aufschluss über vermittelnde Prozessvariablen wie etwa den Gebrauch bestimmter Wortkategorien oder die Veränderungen von Essaymerkmalen im Verlauf der Schreibsitzungen zu geben.

Der Frage nachzugehen, inwieweit sich das Expressive Schreiben auf Aspekte zwischenmenschlicher Interaktionen und dem sozialen Umfeld auswirkt, erscheint im Hinblick auf die Befunde bezüglich der Führungsbenotung im Rahmen dieser Studie mit inhaftierten Jugendlichen lohnend. Sollte sich durch die induzierte Selbstöffnung - vermittelt über Prozesse von Habituation, der Verminderung intrusiver sowie vermeidender Gedanken und einer verbesserte Emotionsregulation - tatsächlich günstigere soziale Interaktionen ergeben (vgl. Horn \& Mehl, 2004), so scheint der aktuelle Forschungsansatz, Verhaltensindikatoren des Soziallebens mittels Mikroaudiorekorder zu erfassen, die Möglichkeit zu bieten, Verhaltensdaten nonreaktiv zu erfassen. Hierbei ließen sich Informationen zum sozialen Umfeld der Studienteilnehmer sowie ihrem Sozial- und Kommunikationsverhalten im Follow-up-Zeitraum genauer analysieren.

Der in dieser Arbeit festgestellte positive Effekt auf die erhobenen Daten des Führungsverhaltens kann für die Ziele und Aufgaben, die sich im Jugendstrafvollzug ergeben, als günstig beurteilt werden. Im Sinne einer zusätzlichen Maßnahme, die sich als gut handhabbar und in den Vollzugsalltag integrierbar erwiesen hat, bei deren Durchführung verschiedene haftspezifische Bedingungen wie etwa die unbedingte Aufklärung über Sinn und Zweck der Maßnahme für den Teilnehmer oder Effekte sozialer Erwünschtheit berücksichtigt werden müssen, kann ES die Möglichkeit eines zusätzlichen Reflektions- und Übungsfeldes für den Inhaftierten im Umgang mit den eigenen Gefühlen und Gedanken bieten.

Zusammenfassend lässt sich feststellen, dass es sich beim Expressiven Schreiben wohl hauptsächlich aufgrund der hohen Ökonomie, was den zeitlichen und personellen Aufwand betrifft, um eine häufig eingesetzte Methode handelt. Aufgrund neuerer metaanalytischer Befunde und den im Rahmen dieser Studie erzielten Ergebnissen müsste jedoch die ursprünglich postulierte positive langfristige Wirkung des ES auf Maße der psychischen und körperlichen Gesundheit tendenziell pessimistisch beurteilt werden. Zukünftige Forschungsbemühungen sollten 
differenzierte Aspekte ausgewählter Parameter genauer explorieren, die einen möglichen Einfluss auf plausible Ergebnismaße zeigen und auf diese Weise die Effektivität von ES zu steigern vermögen.

Auch wenn der hier ermittelte Effekt vom ES auf das Führungsverhalten der Jugendlichen als gering einzuschätzen ist und aufgrund methodischer Einschränkungen nur begrenzt als Wirkung des Schreibens interpretiert werden kann, so ist jedoch vorstellbar, dass ES im Kontext anderer intramuraler Interventionsmaßnahmen im Gruppen- oder Einzelsetting eingesetzt werden und auf diesem Weg einen Beitrag zum Behandlungseffekt haben kann. 


\section{Zusammenfassung}

Ziel der vorliegenden Arbeit war es, zu überprüfen, ob sich die Methode des Expressiven Schreibens (ES) im Kontext eines Jugendstrafvollzugs durchführen lässt und ob sich eine mögliche positive Auswirkung auf den subjektiven Gesundheitsstatus und die psychische Befindlichkeit nachweisen lässt. In einer zusätzlichen vom ursprünglichen Schreibparadigma Pennebakers abweichenden Schreibinstruktion, wurden die Teilnehmer angeleitet, ebenfalls auf emotional expressive Weise, über ihre Straftat(-en) zu schreiben, wobei angenommen wurden, dass sich diese Art der Straftataufarbeitung positiv auf Maße der Normorientierung, Verantwortungsübernahme und Führungsverhalten auswirken kann. Darüber hinaus wurde untersucht, inwieweit ein möglicher Nutzen der Schreibintervention von Persönlichkeitsmerkmalen und inhaltlichen Charakteristika der geschriebenen Essays beeinflusst wird.

Die insgesamt 60 Teilnehmer der offenen Jugendanstalt schrieben an drei aufeinander folgenden Tagen jeweils 20 Minuten über das belastendste Lebensereignis oder die eigene(-n) Straftat(-en) mit der Anweisung, dabei möglichst ihre tiefsten Gefühle und Gedanken auszudrücken. Die Teilnehmer der Kontrollgruppe schrieben auf neutrale Weise über ihren Tagesablauf. Es konnte gezeigt werden, dass sich ES im Vergleich zum sachlichen Schreiben kurzfristig negativ auf die Stimmung auswirkte. Langfristig ergaben sich keine Verbesserung des subjektiven Gesundheitszustandes oder der Maße der psychischen Befindlichkeit. Es zeigte sich lediglich eine signifikante Verbesserung der ausschließlich in den Experimentalgruppen erhobenen Belastungsreaktionen. Beim Maß Zustandsärger konnte eine entgegen gesetzte Veränderung in den beiden Experimentalgruppen beobachtet werden, die aufgrund statistischer Einschränkungen nur unter Vorbehalt interpretiert werden kann. Auch bezogen auf die Maße der Normorientierung ergeben sich keine signifikanten Gruppenunterschiede. Es zeigte sich jedoch, dass die durchschnittlich monatliche Führungsnote im viermonatigen Zeitraum nach der Intervention bei den Teilnehmern, die über ein belastendes Lebensereignis geschrieben haben, signifikant besser war als bei den Jugendlichen der beiden anderen Gruppen.

Ein Einfluss möglicher Moderatorvariablen Antisozialer Persönlichkeitsstörung und Alexithymie blieb weitgehend aus. Die Häufigkeit positiver Emotionsworte und Kognitionsworte erwiesen sich als positiv korreliert mit einer (besseren) Führungsbenotung im ersten Fall und dem gestiegenen Ausmaß an Verantwortungsübernahme im zweiten Fall.

Somit konnte die viel postulierte Annahme, ES habe eine langfristige gesundheitsförderliche Wirkung, im Rahmen dieser Studie mit jugendlichen und heranwachsenden Inhaftierten nicht 
repliziert werden. Die Ergebnisse stehen somit im Einklang neuerer metaanalytischer Forschungsergebnisse, die dem ES im Hinblick auf verschiedene Ergebnismaße - wenn überhaupt - dann nur eine geringe Wirksamkeit bestätigen.

Die Befunde werden unter diesen Aspekten besprochen, in die bisherigen Forschungsergebnisse eingeordnet und bezüglich ihres Aussagegehaltes diskutiert. Schließlich wird besprochen, inwieweit ES sinnvoll im Bereich intramuraler Interventionsmaßnahmen eingesetzt werden kann. 


\section{Literaturverzeichnis}

Abele, A. (1990). Die Erinnerung an positive und negative Lebensereignisse. Untersuchungen zur stimmungsinduzierenden Wirkung und zur Gestaltung der Texte. Zeitschrift für experimentelle und angewandte Psychologie, 37, 181-207.

Abram, K. M., Teplin, L. A. \& Charles, D. R. (2004). Posttraumatic stress disorder in youth in juvenile detention. Archives of General Psychiatry, 61, 403-419.

Abramson, M. F. (1972). The criminalization of mentally disordered behaviour: possible sideeffect of a new mental health law. Hospital and Community Psychiatry, 23, 101-105.

Ames, S. C., Patten, C. A., Werch, C. E., Schroeder, D. R., Stevens, S. R., Fredrickson, P. A., Echols, J. D., Pennebaker, J. W. \& Hurt, R. D. (2007). Nicotine \& Tobacco Research, $9(2), 185-194$.

Anderson, B. \& Farrow, J. A. (1998). Incarcerated adolescents in Washington state. Health services and utilization. Journal of Adolescent Health, 22 (5), 363-367.

Andrews, D. A. \& Bonta, J. (1998). The psychology of criminal conduct. Cincinnati: Anderson Publishing Co.

Andrews, D. A., Zinger, I., Hoge, R. D., Bonta, J., Gendreau, P. \& Cullen, F. T. (1990). Does correctional treatment work? A clinically relevant and psychologically informed metaanalysis. Criminology, 28, 369-404.

Antanowicz, D. \& Ross, R. R. (1993). Essential components of successful rehabilitation programs for offenders. Unpublished paper. Department of Criminology, University of Ottawa.

Bach, M., Bach, D., de Zwaan, M., Serim, M., \& Böhmer, F. (1996). Validierung der deutschen Version der 20-Item Toronto-Alexithymie-Skala bei Normalpersonen und psychiatrischen Patienten. Psychotherapie, Psychosomatik und medizinische Psychologie, 46, 23-28. 
Bagby, R. M., Parker, J. D. \& Taylor, G. J. (1994). The Twenty-Item Toronto Alexithymia Scale I. Item selection and cross validation of the factor structure. Journal of Psychosomatic Research, 38, 23-32.

Basqué, M. (2003). Praxis der Delinquenzbehandlung. In M. Steller, K.-P. Dahle \& M. Basqué (Hrsg.), Straftäterbehandlung - Argumente für eine Revitalisierung in Forschung und Praxis (S. 105-114). Herbolzheim: Centaurus.

Batten, S. V., Follette, V. M., Hall, M. L. R. \& Palm, K. M. (2002). Physical and psychological effects of written disclosure among sexual abuse survivors. Behavior Therapy, 33, 107-122.

Beier, K. M. \& Hinrichs, G. (1995). Psychotherapie mit Straffälligen. Stuttgart: Fischer.

Bosold, C., Prasse, A. \& Lauterbach, O. (2006). Anti-Gewalt-Trainings im Jugendvollzug. Eine bundesweite Bestandsaufnahme. Zeitschrift für Jugendkriminalrecht und Jugendhilfe, 17, 27-37.

Brandewiede, P. (1995). Psychotherapie im Strafvollzug. In K.M. Beier \& G. Hinrichs (Hrsg.), Psychotherapie mit Straffälligen: Standorte und Thesen zum Verhältnis Patient - Therapeut - Justiz (S. 36-41). Stuttgart: G. Fischer.

Brink, J. (2005). Epidemiology of mental illness in a correctional system. Current Opinion in Psychiatry, 18 (5), 536-541.

Burton, C. M., \& King, L. A. (2004). The health benefits of writing about intensely positive experiences. Journal of Research in Personality, 38, 150-163.

Butler, T., Kariminia, A., Levy, M. \& Murphy, M. (2004). The self-reported health status of prisoners in New South Wales. Australian and New Zeeland Journal of Public Health, 28 (4), 344-350. 
Cameron, L. D. \& Nicholls, G. (1998). Expression of stressful experiences through writing: Effects of a self-regulation manipulation for pessimists and optimists. Health Psychology, $17(1), 84-92$.

Campell, N. B. (2003). Emotional disclosure through writing: An intervention to facilitate adjustment to having a child with autism (Doctoral dissertation, University of Mississippi, 2003). Dissertation Abstracts International, 64, 2380.

Campbell, R. S. \& Pennebaker, J. W. (2003). The secret life of pronouns: Flexibility in writing style and physical health. Psychological Science, 14, (1), 60-65.

Cauffman, E. \& Steinberg, L. (2000). (Im)maturity of judgement in adolescence: Why adolescents may be less culpable than adults. Behavioral Sciences and the Law, 18, 741-760.

Chitsabesan, P., Kroll, L., Bailey, S., Kenning, C., Sneider, S., MacDonald, W. \& Theodosiou, L. (2006). Mental health needs of young offenders in custody and in the community. British Journal of Psychiatry, 188, 543-540.

Christensen, A. J., Edwards, D. L., Wiebe, J. S., Benotsch, E. G., McKelvey, L., Andrews, M. \& Lubaroff, D. M. (1996). Effect of verbal self-disclosure on natural killer cell activity: Moderating influence of cynical hostility. Psychosomatic Medicine, 58, 150 155.

Christensen, A. J. \& Smyth, T. W. (1993). Cynical hostility and cardiovascular reactivity during self-disclosure. Psychosomatic Medicine, 55, 193-202.

Cohen, J. (1988). Statistical power analysis for the behavioural sciences $\left(2^{\text {nd }}\right.$ ed.). Hillsdale, NJ: Earlbaum.

Cohen, J. (1992). A power primer. Psychological Bulletin, 112 (1), 155-159. 
Creswell, J. D., Stanton, A. L., Taylor, S. E:, Bower, J. E. \& Sherman, D. K. (2007). Does self-affirmation, cognitive processing, or discovery of meaning explain cancer-related health benefits of expressive writing? Personality and Social Psychology Bulletin, 33 (2), 238-250.

Crow, M. (2000). Psychological and health effects of writing about stress (Doctoral dissertation, Southern Methodist University, 2000). Dissertation Abstracts International, 61, 3316.

Curtis, N. M., Ronan, K. R. \& Borduin, C. M. (2004). Multisystemic treatment: A metaanalysis of outcome studies. Journal of Family Psychology, 18, 411-419.

Dahle, K.-P. (2005). Delinquenzverläufe über die Lebensspanne: Anwendungsperspektiven einer entwicklungsorientierten Sichtweise. In K.-P. Dahle \& R. Volbert (Hrsg.), Entwicklungspsychologische Aspekte der Rechtspsychologie (S. 77-91). Göttingen: Hogrefe.

Dahle, K.-P. (2003a). Probleme bei der Behandlung von Delinquenten. Einführung. In M. Steller, K.-P. Dahle \& M. Basqué, Straftäterbehandlung - Argumente für eine Revitalisierung in Forschung und Praxis (S.177-187). Herbolzheim: Centaurus.

Dahle, K.-P. (2003b). Therapiemotivation inhaftierter Straftäter. In M. Steller, K.-P. Dahle \& M. Basqué, Straftäterbehandlung - Argumente für eine Revitalisierung in Forschung und Praxis (S. 231-250). Herbolzheim: Centaurus.

Dahle, K.-P. \& Steller, M. (1990). Coping im Strafvollzug: Eine Untersuchung zu Haft folgen bei Jugendlichen. Zeitschrift für experimentelle und angewandte Psychologie, 37, 31-51.

Degoratis, L. R. (1977). SCL-90-R, administration, scoring \& procedures manual - I for the $R$ (evised) version. John Hopkins University School of Medicine. 
De Moor, C., Sterner, J., Hall, M., Warneke, C., Gilani, Z., Amato, R. \& Cohern, L. (2002). A pilot study of the effects of expressive writing in a phase II trial of vaccine therapy for metastatic renal cell carcinoma. Health Psychology, 21, 615-619.

Dünkel, F. \& Drenkhahn, K. (2001). Behandlung im Strafvollzug: von "nothing works" zu “something works". In M. Bereswill \& W. Greve (Hrsg.), Forschungsthema Strafvollzug (S. 387-413). Baden-Baden: Nomos.

Earnhardt, J. L., Martz, D. M., Ballard, M. E. \& Curtin, L. (2002). A writing intervention for negative body image: Pennebaker fails to surpass the placebo. Journal of College Student Psychotherapy, 17, 19 - 35.

Eells, J. E. (2007). Expressive writing, relationships, and health. (Doctoral dissertation, Columbia, Universitiy of Missouri, 2007). Dissertation Abstracts International, 67, 6771.

Egg, R. (2003). Sozialtherapeutische Einrichtungen im Strafvollzug - Konzeptionelle und strukturelle Probleme. In M. Steller, K.-P. Dahle \& M. Basqué, Straftäterbehandlung - Argumente für eine Revitalisierung in Forschung und Praxis (S. 189204). Herbolzheim: Centaurus.

Egg, R. (1990). Sozialtherapeutische Behandlung und Rückfälligkeit im längerfristigen Vergleich. Monatsschrift für Kriminologie und Strafrechtsreform, 73, 358-368.

Egg, R. (1986). Zwangstherapie. Zur Bedeutung der Freiwilligkeit bei der Durchführung therapeutischer Maßnahmen. Verhaltenstherapie und psychosoziale Praxis, 1, 35-41.

Egg. R., Pearson, F. S., Cleland, C. M. \& Lipton, D. S. (2001). Evaluation von Straftäterbehandlungsprogrammen in Deutschland: Überblick und Meta-Analyse. In G. Rehn, B. Wischka, F. Lösel \& M. Walter (Hrsg.), Behandlung „gefährlicher Straftäter“. Grundlagen, Konzepte, Ergebnisse (S. 321-345). Herbolzheim: Centaurus. 
Egloff, B. \& Krohne, H.W. (2002). PANAS. Positive and Negative Affect Schedule. In E. Brähler, J. Schumacher \& B. Strauß (Hrsg.), Diagnostische Verfahren in der Psychotherapie (S. 264-266). Göttingen: Hogrefe.

Ehlers, A. (2000). Posttraumatische Belastungsstörungen. (Bd. 8). Göttingen: Hogrefe.

Eisenberg, A.R., Cumberland, A., \& Spinrad, T. L. (1998). Parental socialisation of emotion. Psychology Inquiry, 9, 241-273.

Endres, J. (2004). Prognosebegutachtung. In W. Pecher (Hrsg.) Justizvollzugspsychologie in Schlüsselbegriffen (S. 177-192). Stuttgart: Kohlhammer.

Enzmann, D. \& Greve, W. (2001). Strafhaft für Jugendliche: Soziale und individuelle Bedingungen von Delinquenz und Sanktionierung. In M. Bereswill \& W. Greve (Hrsg.), Forschungsthema Strafvollzug (S.109-146). Baden-Baden: Nomos.

Enzmann, D. \& Raddatz, S. (2005). Substanzabhängigkeit jugendlicher und heranwachsender Inhaftierter. In K.-H. Dahle \& R. Volbert (Hrsg.), Entwicklungspsychologische Aspekte der Rechtspsychologie (S. 150-172). Göttingen: Hogrefe.

Esterling, B. A., Antoni, M. H., Fletcher, M. A., Margulies, S. \& Schneidermann, N. (1994). Emotional disclosure through writing or speaking modulates latent Epstein-Barr virus antibody titers. Journal of Consulting and Clinical Psychology, 62 (1), 130-140.

Evans, C. M. G. (2000). The effects of writing about traumatic experiences on adolescents identified as emotionally disturbed (ED) (Doctoral dissertation, Texas Woman's University, 2000). Dissertation Abstracts International, 60, 3269.

Fazel, S. \& Danesh, J. (2002). Serious mental disorder in 23000 prisoners: a systematic review of 62 surveys. Lancet, 359, 545-550.

Fazel, S. \& Lubbe, S. (2005). Prevalence and characteristics of mental disorders in jails and prisons. Current Opinion in Psychiatry, 18 (5), 550-554. 
Forsten, M. T. (1992). Disclosure of traumatic events utilization a writing task:

Immunological, physiological, and psychological consequences in a psychiatric population (Doctoral dissertation, Auburn Universitiy, 1992). Dissertation Abstracts International, 52, 3904.

Francis, M. E. \& Pennebaker, J. W. (1992). Putting stress into words: The impact of writing on physiological, absentee, and self-reported emotional well-being measures. American Journal of Health Promotion, 6, 280-287.

Frattarolli, J. (2006). Experimental disclosure and its moderators: A meta-analysis. Psychological Bulletin, 132, 823-865.

Freese, R. (2005). Das Behandlungskonzept der Klinik. Klinik für Forensische Psychiatrie Haina [online Ressource].Verfügbar unter: psych-haina.de / kffp /html/ behkonzept.html.

Frisina, P.G., Borod, J. C. \& Lepore, S. J. (2004). A meta-analysis of the effects of written emotional disclosure on the health outcomes of clinical populations. Journal of Nervous and Mental Disease, 192 (9), 629-634.

Garrett, P. (1985). Effects of residential treatment of adjucated delinquents: A metaanalysis. Journal of Research in Crime and Delinquency, 22, 287-308.

Geissler, I. (1991). Ausbildung und Arbeit im Jugendstrafvollzug. Haftverlaufs- und Rückfallanalyse (Kriminologische Forschungsberichte aus dem Max-Planck-Institut für ausländisches und internationales Strafrecht, Bd. 44). Freiburg i. Br.: Max-PlanckInstitut für ausländisches und internationales Strafrecht.

Gidron, Y., Duncon, E., Lazar, A., Biderman, A., Tandeter, H. \& Shvartzman, P. (2002). Effects of guided written disclosure of stressfull experiences on clinic visits and symptoms in frequent clinic attenders. Familiy Practice, 19 (2), 161-166. 
Gidron, Y., Peri, T., Connolly, J. F., \& Shalev, A. Y. (1996). Written emotional disclosure in posttraumatic stress disorder: Is it beneficial for the patient? Journal of Nervous and Mental Disease, 184, 505-507.

Goddard, L. Dritschel, B. \& Burton, A. (1996). Role of autobiographical memory in social problem solving and depression. Journal of Abnormal Psychology, 105, 609-616.

Golzari, M., Hunt, S. J. \& Anoshiravani, A. (2006). The health status of youth in juvenile detention facilities. Journal of Adolescent Health, 38 (6), 776-782.

Gottfredson, M. \& Hirschi, T. (1990). A general theory of crime. Stanford, CA: Stanford University Press.

Gottschalk, R., Davidson II, W. S., Gensheimer, L. K. \& Mayer, J. P. (1987). Communitybased interventions. In H. C. Quay (Ed.), Handbook of juvenile delinquency (pp. 266289). New York: Wiley.

Greenberg, M. A. \& Stone, A. A (1992). Emotional disclosure about traumas and its relation to health: Effects of previous disclosure and trauma severity. Journal of Personality and Social Psychology, 63 (1), 75-84.

Greenberg, M. A., Wortmann, C. B. \& Stone, A. A. (1996). Emotional expression and physical health: Rivising traumatic memories or fostering self-regulation? Journal of Personality and Social Psychology, 71 (3), 588-602.

Greve, W., Hosser, D. \& Pfeiffer, C. (1996). Gefängnis und die Folgen. Identitätsentwicklung und kriminelles Handeln während und nach Verbüßung einer Jugendstrafe. JuStBericht Nr. 1. Hannover: KFN- Forschungsberichte Nr. 64.

Hagell, A. (2002). The mental health of young offenders. - Bright Futures: Working with vulnerable young people. Mental Health Foundation, London. 
Hagemann, O. (2003). Introspektion und Empathie in der Arbeit mit Strafgefangenen. In: C. Kumbruck, M. Dick \& H. Schulze (Hrsg.). Arbeit-Alltag-Psychologie. Über den Bootsrand geschaut. Heidelberg: Asanger Verlag.

Harber, K. D. \& Pennebaker, J. W. (1992). Overcoming traumatic memories. In S.-A. Christianson (Ed.), The handbook of emotion and memory (pp. 359-387). Hillsdale, NJ: Lawrence Erlbaum Associates.

Harris, A. H. (2006). Does expressive writing reduce health care utilization? A meta-analysis of randomized trials. Journal of Consulting and Clinical Psychology, 74 (2), 243-252.

Harris, F., Hek, G. \& Condon, L. (2006). Health needs of prisoners in England and Wales: the implications for prison healthcare of gender, age and ethnicity. Health and Social Care in the Community, 15 (1), 56-66.

Harris, A. H. S., Thoresen, C. E., Humphreys, K., \& Faul, J. (2004). Effects of expressive writing on lung function in adult asthmatics. Poster presented at the $112^{\text {th }}$ Annual Convention of the American Psychological Association, Honululu, HI.

Harris, A. H. S., Thoresen, C. E., Humphreys, K., \& Faul, J. (2005). Does writing affect asthma? A randomized trial. Psychosomatic Medicine, 67, 130-136.

Harrist, S., Carlozzi, B. L., McGovern, A. R. \& Harrist, A. W. (2007). Benefits of expressive writing and expressive talking about life goals. Journal of Research in Personality, 41 (4), 923-930.

Hautzinger, M. \& Bailer, M.(1993a). Allgemeine Depressions Skala (ADS). Weinheim: Beltz Test GmbH.

Hautzinger, M. \& Bailer, M. (1993b). Das Inventar depressiver Symptome (IDS). Weinheim: Beltz Test GmbH. 
Heekerens, H.-P. (2006). Die Multisystemische Therapie. Ein evidenz-basiertes Verfahren zur Rückfallprophylaxe bei Jugendlichendelinquenz. Zeitschrift für Jugendkriminalität und Jugendhilfe, 17 (2), 163-171.

Hein, K., Cohen, M. I. \& Litt, I. F. (1980). Juvenile detention: another boundary issue for physicians. Pediatrics, 66 (29), 239-245.

Hinrichs, G. (1993). Zur therapeutischen Problematik mangelnder Tatverarbeitung bei Kapitaldelikten. Zeitschrift für Strafvollzug und Straffälligenhilfe, 3, 159-162.

Hinrichs, G. (1994). Was ist „Tatverarbeitung“ und wozu kann sie dienen?. Monatsschrift für Kriminologie und Strafrechtsreform, 77 (2), 95-101.

Hinrichs, G. (1995). Zur intramuralen Psychotherapie junger Straftäter. In K.M. Beier \& G. Hinrichs (Hrsg.), Psychotherapie mit Straffälligen: Standorte und Thesen zum Verhältnis Patient - Therapeut - Justiz (S. 71-78). Stuttgart: G. Fischer.

Hinrichs, G., Behnisch, A., Krull, K. \& Reimers, S. (2000). Therapiemotivation junger Strafgefangener. Zeitschrift für Kinder- und Jugendpsychiatrie und Psychotherapie, 28 (4), 255-262.

Hochweber, H. (2004). Jugendvollzug. In W. Pecher (Hrsg.) Justizvollzugspsychologie in Schlüsselbegriffen (S. 117-129). Stuttgart: Kohlhammer.

Holmes, T. H., \& Rahe, R. H. (1967). The Social Readjustment Rating Scale. Journal of Psychosomatic Research, 11, 213-218.

Horn, A. B. (2004). Depressionsprävention durch Förderung der Emotionsregulation: Expressives Schreiben für Jugendliche. Bd. 7, Reihe Berichte aus dem DFGGraduiertenkolleg „Lebensstile, Soziale Differenzen, Gesundheitsförderung“. Berlin: Logos.

Horn, A. B. \& Mehl, M. R. (2004). Expressives Schreiben als Copingtechnik: Ein Überblick über den Stand der Forschung. Verhaltenstherapie, 14, 274-283. 
Hosser, D. (2004). Jugendstrafvollzug und die Folgen. KFN-Newsletter, 1, 11-17.

Hosser, D. \& Greve, W. (1999). Gefängnis und die Folgen. Identitätsentwicklung und kriminelles Handeln während und nach der Verbüßung einer Jugendstrafe. Das Erhebungsinstrument der standardisierten Befragung. Hannover: KFN-Forschungsberichte Nr. 77.

Hughes, K. N. (2007). Expressive writing and breast cancer: Outcomes and linguistic analyses. (Doctoral dissertation, Utah State University, 2007). Dissertation Abstracts International, 68, 1309.

Izzo, R. L. \& Ross, R. R. (1990). Meta-analysis of rehabilitation programs for juvenile delinquents. A brief report. Criminal Justice and Behavior, 17, 134-142.

Kelley, J. E., Lumley, M. A. \& Leysen, J. C. C. (1997). Health effects of emotional disclosure in rheumatoid arthritis patients. Health Psychology, 16, 331-340.

Kerner, H.-J. \& Janssen, H. (1996). Rückfall nach Verbüßung einer Jugendstrafe. Langfristverlauf im Zusammenspiel von soziobiographischer Belastung und krimineller Karriere. In Kerner, H.-J., Dolde, G. \& Mey, H.-G. (1996), Jugendstrafvollzug und Bewährung (S.137-219). Mönchengladbach: Forum Verlag Godesberg.

King, L. A. (2001). The health benefits of writing about life goals. Personality and Social Psychology Bulletin, 27, 789-807.

King, L. A. \& Miner, K. N. (2000). Writing about the perceived benefits of traumatic events: Implications for physical health. Personality and Social Psychology Bulletin, 26, 220230.

Klaghofer, R. \& Brähler, E. (2000). Konstruktion und teststatistische Prüfung einer Kurzform der SCL-90-R. Zürich, Universitätsspital.

Klein, K. \& Boals, A. (2001). Expressive writing can increase working memory capacity. Journal of Experimental Psychology: General, 130, 520-533. 
Kloss, J. D. \& Lisman, S. A. (2002). An exposure-based examination of the effects of written emotional disclosure. British Journal of Health Psychology, 7, 31-46.

Konrad, N. (2004). Vollzugspsychiatrie. In W. Pecher (Hrsg.), Justizvollzugspsychologie in Schlüsselbegriffen (S. 321-334). Stuttgart: Kohlhammer.

Krohne, H. W., Egloff, B., Kohlmann, C. W. \& Tausch, A. (1996). Untersuchungen mit einer deutschen Version der „Positive and Negative Affect Schedule“ (PANAS). Diagnostica, 42, 139-156.

Kröner-Herwig, B., Linkemann \& A., Morris, L. (2004). Selbstöffnung beim Schreiben über belastende Lebensereignisse. Ein Weg in die Gesundheit? Zeitschrift für Klinische Psychologie und Psychotherapie, 33 (3), 183-195.

Krupinski, M., Schöchlin, C., Fischer, A. \& Nedopil, N. (1998). Annäherung an ambulante Therapie bei Straffälligen. In H.-L. Kröber \& K.-P. Dahle (Hrsg.), Sexualstraftaten und Gewaltdelinquenz (S. 169-174). Heidelberg: Kriminalistik-Verlag.

Lader, D., Singleton, N. \& Meltzer, H. (2000). Psychiatric Morbidity among Young Offenders in England and Wales. Office for National Statistics, London.

Lange, A., van de Ven, J.-P., \& Schrieken, B. (2003). Interapy: Treatment of post-traumatic stress via the internet. Cognitive Behaviour Therapy, 32, 110-124.

Laux, L., Glanzmann, P., Schaffner, P. \& Spielberger, C. D. (1981). Das State-Trait-Angstinventar (STAI). Weinheim: Beltz Test GmbH.

Lepore, S. J. (1997). Expressive writing moderates the relation between intrusive thoughts and depressive symptoms. Journal of Personality and Social Psychology, 73, 10301037. 
Lepore, S. J., Greenberg, M. A., Bruno, M. \& Smyth, J. M. (2002). Expressive writing and health: Self-regulation of emotion-related experience, physiology, and behavior. In S. J. Lepore \& J. M. Smyth (Eds.), The writing cure: How expressive writing promotes health and emotional well being (pp. 99-117). Washington, DC: American Psychological Association.

Lepore, S. J. \& Smyth, J. M. (2002). The writing cure: How expressive writing promotes health and emotional well being. Washington, DC: American Psychological Association.

Lewand, M. (2005). Persönlichkeits- und Delinquenzentwicklung. In K.-P. Dahle \& R. Volbert (Hrsg.), Entwicklungspsychologische Aspekte der Rechtspsychologie (S. 129138). Göttingen: Hogrefe.

Linkemann, A. (2001). Schreiben über belastende Lebensereignisse und dessen Einfluss auf körperliches und psychisches Wohlbefinden. Unveröffentlichte Dissertation, GeorgAugust-Universität Göttingen.

Lipsey, M. W. (1992). The effect of treatment on juvenile delinquents: Results from metaanalysis. In F. Lösel, D. Bender \& T. Bliesener (Eds.), Psychology and law. International perspectives (pp. 131-143). Berlin: de Gruyter.

Lösel, F. (2003). Meta-analytische Beiträge zur wiederbelebten Diskussion des Behandlungsgedankens. In M. Steller, K.-P. Dahle \& M. Basqué (Hrsg.), Straftäterbehandlung. Argumente für eine Revitalisierung in Forschung und Praxis (S. 13-34). Herbolzheim: Centaurus.

Lösel, F. (1993). Sprechen Evaluationsergebnisse von Meta-Analysen für einen frischen Wind in der Straftäterbehandlung? In R. Egg (Hrsg.), Sozialtherapie in den 90er Jahren. Gegenwärtiger Stand und Entwicklung im Justizvollzug (S. 21-31). Wiesbaden: Kriminologische Zentralstelle.

Lumley, M. A. (2004). Alexithymia, emotional disclosure, and health: A program of research. Journal of Personality, 72 (2), 1271-1300. 
Maercker, A. \& Schützwohl, M. (1998). Erfassung von psychischen Belastungsfolgen: Die Impact of Events Skala - revidierte Version (IES-R). Diagnostica, 44, 130-144.

Mann, T. (2001). Effects of future writing and optimism on health behaviors in HIV-infected women. Annals of Behavioral Medicine, 23, 26-33.

Meads, C. \& Nouwen, A. (2005). Does emotional disclosure have any effects? A systematic review of the literature with meta - analyses. International Journal of Technology Assessment in health Care, 21, 153-164.

Mehl, M. R. \& Pennebaker, J. W. (2000). The effects of emotional writing on students' daily social environment and natural conversations. Unveröffentlichtes Manuskript.

Mehl, M. R. \& Pennebaker, J. W. (2003). The sounds of social life: A psychometric analysis of students' daily social environments and natural conversations. Journal of Personality and Social Psychology, 84, 857-870.

Mey, H.-G. (1996). Rechtlicher Bezugsrahmen für Jugendstrafe und Jugendstrafvollzug. In H.-J. Kerner, G. Dolde \& H.-G. Mey (Hrsg.), Jugendstrafvollzug und Bewährung. Analysen zum Vollzugsverlauf und zur Rückfallentwicklung (S.117-136). Bonn: Forum Verlag Godesberg.

Mitchell, J. \& Palmer, E. J. (2004). Evaluating the "Reasoning and Rehabilitation" Program for Young Offenders. Journal of Offender Rehabilitation, 39 (4), 31-45.

Mogk, C., Otte, S., Reinhold-Hurley, B. \& Kröner-Herwig, B. (2006). Health effects of expressive writing on stressful or traumatic experiences - a meta-analysis. GMS Psychosomatic Medicine, 3.

Murray, E. J., Lamnin, A. D. \& Carver, C. S. (1989). Emotional expression in written essays and psychotherapy. Journal of Social and Clinical Psychology, 8, 414-429.

Murray, E. J. \& Segal, D. L. (1994). Emotional processing in vocal and written expression of feelings about traumatic experience. Journal of Traumatic Stress, 7 (3), 391-405. 
Neuner, F., Schauer, M., Klaschik, C., Karunakara, U. \& Elbert, T. (2004). A comparison of narrative exposure therapy, supportive counseling, and psychoeducation for treating posttraumatic stress disorder in an African refugee settlement. Journal of Consulting and Clinical Psychology, 72 (4), 579-587.

Obrist, C. \& Werdenich, W. (2004). Drogentäter. In W. Pecher (Hrsg.), Justizvollzugspsychologie in Schlüsselbegriffen (S. 40-50). Stuttgart: Kohlhammer.

Ohlemacher, T. (2000). Anti-Aggressivitätstraining und Legalbewährung. Versuch einer Evaluation. Hannover: KFN-Forschungsberichte Nr. 83.

Ortmann, R. (1993). Zur Evaluation der Sozialtherapie. Zeitschrift für die gesamte Strafrechtswissenschaft, 106, 782-821.

Oswald, M. E. \& Bütikofer, A. (2003a). Wissenschaftliche Evaluation des Modellversuches „Tataufarbeitung und Wiedergutmachung (TaWi)-Berner Modell“, Umsetzungsphase, Schlussbericht. Forschungsberichte aus dem Psychologischen Institut der Universität Bern, 2003-1.

Oswald, M. E. \& Bütikofer, A. (2003b). Entwicklung einer Pilotversion zur Messung von Verantwortungsübernahme bei Straftäter/innen: Forschungsbericht. Forschungsberichte aus dem Psychologischen Institut der Universität Bern, 2003-3.

Otto, M. (2003). Soziales Training. Konzepte, Rahmenbedingungen und Effekte. In M. Steller, K.-P. Dahle \& M. Basqué (Hrsg.), Straftäterbehandlung - Argumente für eine Revitalisierung in Forschung und Praxis (S. 115-133). Herbolzheim: Centaurus.

Paez, D., Basabe, N., Valdoseda, M., Velasco, C. \& Iraurgi, I. (1995). Confrontation, inhibition, alexithymia, and health. Emotion, disclosure, and health (pp. 195-222). Washington: APA.

Paez, D., Velasco, C. \& Ganzalez, J. L. (1999). Expressive writing and the role of alexithymia as a dispositional deficit in self-disclosure and psychological health. Journal of Personality and Social Psychology, 77, 630-641. 
Park, C. L. \& Blumberg, C. J. (2002). Disclosing trauma through writing: Testing the meaning-making hypothesis. Cognitive Therapy and Research, 26 (5), 597-616.

Parker, J. D. A., Taylor, G. J. \& Bagby, R. M. (2002). The 20-Item Toronto Alexithymia Scale III. Reliability and factorial validity in a community population. Journal of Psychosomatic Research, 5, 269-275.

Pasupathi, M. (2007). Telling and the remembered self: Linguistic differences in memories for previously disclosed and previously undisclosed events. Memory, 15 (3), 258-270.

Person, F. S. \& Lipton, D. S. (1999, November). The Effectiveness of educational and vocational programs: CDATE Meta-analyses. Vortrag beim Jahrestreffen der American Society of Criminology, Toronto.

Pecher, W. (1999). Tiefenpsychologisch orientierte Psychotherapie im Justizvollzug. Pfaffenweiler: Centaurus.

Pecher, W. (2004). Resozialisierung. In W. Pecher (Hrsg.), Justizvollzugspsychologie in Schlüsselbegriffen (S. 215-224). Stuttgart: Kohlhammer.

Pennebaker, J. W. (1988). Confiding traumatic experiences and health. In S. Fisher \& J. Reason (Eds.), Handbook of life stress, cognition and health. Chichester: John Wiley \& Sons.

Pennebaker, J. W. (1989). Confession, inhibition, and disease. In L. Berkowitz (Ed.), Advances in Experimental and Social Psychology 22, (pp. 211-244). New York: Academic Press.

Pennebaker, J. W. (1993). Putting stress into words: Health, linguistic, and therapeutic implications. Behavior Research Therapy, 3 (6), 539-548.

Pennebaker, J. W. (1999). The effects of traumatic disclosure on physical and mental health: the values of writing and talking about upsetting events. International Journal of Emergency Mental Health, 1, 9-18. 
Pennebaker, J. W. (2002). Emotion, Disclosure \& Health. Washington, DC: American Psychological Association.

Pennebaker, J. W., Barger, S. D. \& Tiebout, J. (1989). Disclosure of traumas and health among holocaust survivors. Psychosomatic Medicine, 51, 577-589.

Pennebaker, J. W., \& Francis, M. E. (1996). Cognitive, emotional, and language processes in disclosure. Cognition and Emotion, 10 (6), 601-626.

Pennebaker, J. W., Francis, M. E. \& Booth, R. J. (2001). Linguistic inquiry and word count (LIWC). Mahwah, NJ: Erlbaum Publishers.

Pennebaker, J. W. \& Graybeal, A. (2001). Patterns of natural language use: Disclosure, personality, and social integration. Current Directions in Psychological Science, 10, 90-93.

Pennebaker, W. J., Kiecolt-Glaser, J. K. \& Glaser, R. (1988). Disclosure of Traumas and immune function: Health implications for psychotherapy. Journal of Consulting and Clinical Psychology, 56 (2), 239-245.

Pennebaker, J. W., Mayne, T. J. \& Francis, M. E. (1997). Linguistic predictors of adaptive bereavement. Journal of Personality and Social Psychology, 72 (4), 863-871.

Pennebaker, J. W. \& Seagal, J. (1999). Forming a story: The health benefits of narrative. Journal of Clinical Psychology, 55, 1243-1254.

Petrie, K. J., Booth, R. J. \& Pennebaker, J. W. (1998). The immunological effects of thought suppression. Journal of Personality and Social Psychology, 75, 1264-1272.

Petrie, K. J., Booth, R. J., Pennebaker, J. W., Davison, K. P. \& Thomas, M. G. (1995). Disclosure of trauma and immune response to a Hepatitis B vaccination program. Journal of Consulting and Clinical Psychology, 63 (5), 787-792. 
Rauchfleisch, W. (2003) Behandlungsstrategien ganz gezielt der Realität der Delinquenten anpassen. In M. Steller, K.-P. Dahle \& M. Basqué (Hrsg.), Straftäterbehandlung Argumente für eine Revitalisierung in Forschung und Praxis (S. 261-265). Herbolzheim: Centaurus.

Richards, J. M., Beal, W.E., Seagal, J.D. \& Pennebaker, J.W. (2000). Effects of disclosure of traumatic events on illness behavior among psychiatric prison inmates. Journal of Abnormal Psychology, 109 (1), 156-160.

Robinson, D. (1995). The impact of cognitive skills training on post-release recidivism among Canadian federal offenders. No. R-41. Research Branch. Ottawa: Correctional Service Canada.

Robinson, D. \& Porporino, F. J. (2001). Programming in cognitive skills: The Reasoning and Rehabilitation programme. In C. R. Hollin (Ed.), Handbook of offender assessment and treatment (pp. 179-193). Chichester: Wiley.

Rössner, D. (2005). Täter-Opfer-Ausgleich. Förderung der sozialen Verantwortung und Integration durch Opferbezug im Jugendstrafvollzug. Zeitschrift für Jugendkriminalität und Jugendhilfe, 16 (1), 30-34.

Ross, R. R. \& Fabiano, E. A. (1985). Time to think: A cognitive model of delinquency prevention and offender rehabilitation. Johnson City, TN: Institute of Social Sciences and Arts.

Ross, R. R., Fabiano, E. A. \& Ross, R. D. (1986). Reasoning and rehabilitation: A handbook for teaching cognitive skills. Ottawa: Cognitive Center.

Traue, H. C., (1998). Emotion und Gesundheit. Berlin: Spektrum Akademischer Verlag.

Schoutrop, J. A., Lange, A., Hanewald, G., Dadidovich, U. \& Salomon, H. (2002). Structured writing and processing major stressful events: A controlled trial. Psychotherapy and Psychosomatics, 71, 151-157. 
Schwartz, L., \& Drotar, D. (2004). Effects of written emotional disclosure on caregivers of children and adolescents with chronic illness. Journal of Pediatric Psychology, 29, 105-118.

Schwenkmezger, P., Hodapp, V. \& Spielberger, C. D. (1992). Das State-Trait-Ärgerausdrucks-Inventar (STAXI). Göttingen: Hans Huber.

Sharsky, M. (1997). Emotional consequences of written expression of traumatic and positive experience (Doctoral dissertation, Hofstra University, 1997). Dissertation Abstracts International, 58, 2140.

Shelden, D. (2001). Emotional disorders in young offenders. Journal of Nursing Scholarship, 33 (3), 259-263.

Sheese, B. E., Brown, E. L., \& Graziano, W. G. (2004). Emotional expression in cyberspace: Searching for moderators of the Pennebaker disclosure effect via e-mail. Health Psychology, 23, 457-464.

Simons, D. (1996). „Tataufarbeitung““ - Diagnostik mit beschränkter Haftung! Zeitschrift für Strafvollzug und Straffälligenhilfe, 1, 10-14.

Simourd, D. J. (1997). The Criminal Sentiments Scale -Modified and Pride in Delinquency scale: Psychometric properties and construct validity of two measures of criminal attitudes. Criminal Justice and Behavior, 24 (1), 52-70.

Simourd, D. J., van de Veen, J. (1999). Assessment of criminal attitudes: Criterion-related validity of the Criminal Sentiments Scale-modified and Pride in Delinquency Scale. Criminal Justice and Behavior, 26 (1), 90-106.

Simourd, D. J. (2002). The future of criminal attitudes research and practice. Criminal Justice and Behavior [Special Edition], 29 (4), 427-446.

Sloan, D. M. \& Marx, B. P. (2004a). A closer examination of the structured written disclosure procedure. Journal of Consulting and Clinical Psychology, 72, 165-176. 
Sloan, D. M. \& Marx, B. P. (2004b). Taking pen to hand: evaluating theories underlying the written disclosure paradigm. Clinical Psychology: Science and Practice, 11, 121-137.

Smyth, J. M. (1998). Written emotional expression: Effect sizes, outcome types, and moderating variables. Journal of Consulting and Clinical Psychology, 66 (1), 174-184.

Smyth, J. M., True, N. \& Souto, J. (2001). Effects of writing about traumatic experiences: The necessity for narrative structure. Journal of Social \& Clinical Psychology, 20, 161172.

Snyder, D. K., Gordon, K. C. \& Baucom, D. H. (2004). Treating affair couples: Extending the written disclosure paradigm to relationship trauma. Clinical Psychology: Science and Practice,11, 155-161.

Solano, L., Donati, V., Pecci, F., Persichetti, S. \& Colaci, A. (2003). Post-operative course after papilloma resection: Effects of written disclosure of the experience in subjects with different alexithymia levels. Psychosomatic Medicine, 65, 477-484.

Sonnen, B.-R. (2002). Jugendstrafvollzug in Deutschland. Rechtliche Rahmenbedingungen und kriminalpolitische Entwicklungen. In M. Bereswill \& T. Höynck (Hrsg.), Jugendstrafvollzug in Deutschland. Grundlagen, Konzepte, Handlungsfelder (S. 57- 78). Mönchengladbach: Forum Verlag Godesberg.

Spera, S. P. \& Buhrfeind, E. D., Pennebaker, J. W. (1994). Expressive writing and coping with job loss. Academy of Management Journal, 37 (3), 722-733.

Spielberger, C. D. (1983). Manual for the State-Trait-Anxiety Inventory (STAXI). Palo Alto, CA: Consulting Psychologist Press.

Spielberger, C. D., Gorsuch, R. L. \& Lushene, R. E. (1970). Manual for the State-Trait Anxiety Inventory. Palo Alto, Calif.: Consulting Psychologists Press. 
Steller, M. (2003). Behandlung und Behandlungsforschung. In M. Steller, K.-P. Dahle \& M. Basqué (Hrsg.), Straftäterbehandlung - Argumente für eine Revitalisierung in Forschung und Praxis (S. 3-12). Herbolzheim: Centaurus.

Stemmer-Lück, M. (1980). Die Behandlungsindikation bei Straffälligen. Göttingen: Otto Schwarz.

Stöver, H. (2002). Drug substitution treatment and needle exchange programs in German and European prisons. Journal of Drug Issues, 32 (2), 573-595.

Stöver, H. (1999). DrogenkonsumentInnen im Justizvollzug. Die Gleichzeitigkeit von Hilfe und Kontrolle als Konflikt- Bewältigungsversuche. In W. Nickolai \& R. Reindl (Hrsg.), Renaissance des Zwangs: Konsequenzen für die Straffälligenhilfe (S. 91-117). Freiburg im Breisgau: Lambertus.

Stone, A., Smyth, J., Kaell, A. \& Hurewitz, A. (2000). Structured writing about stressful events: Exploring potential psychological mediators of positive health effects. Health Psychology, 19, 619-624.

Studtmann-Pieper, M. (n.d.). Expressives Schreiben im offenen Jugendstrafvollzug - Eine Inhaltsanalyse. Unveröffentlichte Diplomarbeit, Georg-August Universität, Göttingen.

StVollzG-Strafvollzugsgesetz mit Verwaltungsvorschriften (17. Auflage3). (2005). München: C.H. Beck.

Suhling, S. (2005). Lebensziele junger Männer im Strafvollzug. Theoretische und empirische Argumente aus aktionaler Entwicklungsperspektive. Baden-Baden: Nomos.

Suhling, S. (2005) Motivation ist alles? Formen und Bedingungen von Veränderungs- und Behandlungsbereitschaft bei Inhaftierten. Zeitschrift für Jugendkriminalrecht und Jugendhilfe, 16 (4), 385-396. 
Swanbon, T. (1999). The physical and psychological health effects of self-disclosure in homosexual males (Doctoral dissertation, California School of Professional Psychology, 1999). Dissertation Abstracts International, 60, 5794).

Sykes, G. M. \& Matza, D. (1957). Techniques of neutralization: A theory of delinquency. American Sociological Review, 22 (6), 664-670.

Tatti, A. F. (2007). An examination of the written disclosure paradigm utilizing the Internet. (Doctoral dissertation, Pace University, 2007). Dissertation Abstracts International, 67, 6747.

Teplin, L. A., Abram, K. M. \& McClelland, G. M. (2002). Psychiatric disorders in youth in juvenile detention. Archives of General Psychiatry, 59, 1133-1143.

Timmons-Mitchell, J., Bender, M. B., Kishna, M. A. \& Mitchell, C. C. (2006). An independent effectiveness trial of Multisystemic Therapy with juvenile justice youth. Journal of Clinical Child and Adolescent Psychology, 35 (2), 227-236.

Tromp, S. N. (1998). The use of self-guided writing therapy as an intervention for trauma: A sample of incarcerated women (Doctoral dissertation, University of Arizona, 1998). Dissertation Abstracts International, 58, 3936.

Ullrich, P. M. \& Lutgendorf, S. K. (2002). Journaling about stressful events: Effects of cognitive processing and emotional expression. Annual Behavior and Medicine, 24 (3), 244-250.

von Zerssen, D. (1976). Die Beschwerden-Liste. Manual. Weinheim: Beltz.

Vreugdenhil, C., Doreleijers, T. A. H. \& Vermeiren, R. (2004). Psychiatric disorders in a representative sample of incarcerated boys in The Netherlands. Journal of the American Academy of Child and Adolescent Psychiatry, 43, 97-104. 
Walkenhorst, P. (2002). "Gute Schulen” im Jugendstrafvollzug - Jugendstrafvollzug als "gute Schule”. Überlegungen zu Voraussetzungen und Möglichkeiten. In M. Bereswill \& T. Höynck (Hrsg.), Jugendstrafvollzug in Deutschland. Grundlagen, Konzepte, Handlungsfelder (S. 319-355). Godesberg: Forum.

Walker, B. L., Nail, L. M., \& Croyle, R. T. (1999). Does emotional expression make a difference in reactions to breast cancer ? Oncology Nursing Forum, 26, 1025-1032.

Watson, D., Clark, L. A. \& Tellegen, A. (1988). Development and validation of brief measures of positive and negative affect: The PANAS scales. Journal of Personality and Social Psychology, 54, 1063-1070.

Watson, D. \& Tellegen, A. (1985). Toward a consensual structure of mood. Psychological Bulletin, 98, 219-235.

Waxweiler, R. (1980). Psychotherapie im Strafvollzug. Weinheim: Beltz.

Weidner, J. (1990). Anti-Aggressivitäts-Training für Gewalttäter: Ein deliktspezifisches Behandlungsangebot im Jugendvollzug. Mönchengladbach: Forum.

Wendt, C. \& Hinrichs, G. (1993). Wie beurteilen jugendpsychiatrische stationäre Patienten und ihre Therapeuten im Nachhinein die stattgehabte Einzelpsychotherapie? Zeitschrift für Kinder- und Jugendpsychiatrie, 21, Supplement 1,63.

Winslett, P. W. (2007). Positive and negative emotional writing in adolescence: Gender differences, benefits, and effects on self-disclosure and intimacy. (Doctoral dissertation, University of Mississippi, 2007). Dissertation Abstracts International, 67, 7395.

Wittchen, H.-U., Schramm, E. Zaudig, M. \& Unland, H. (1993). Strukturiertes Klinische Interview für DSM-III-R Achse II, Persönlichkeitsstörungen. Göttingen: Hogrefe.

Zakowski, S. G., Ramati, A., Morton, C., Johnson, P., \& Flanigan, R. (2004). Written 
emotional disclosure buffers the effects of social constraints on distress in cancer patients. Health Psychology, 23, 555-563. 


\section{Anhang}

Anhang A

Anhang B

Anhang C
Informationsmaterial und Instruktion
A1 Allgemeine Instruktion
A2 Einverständniserklärung des Teilnehmers
A3 Instruktion Gruppe BE
A4 Instruktion Gruppe TA
A5 Instruktion Gruppe KG

\section{Fragebögen}

$\begin{array}{ll}\text { B1 } & \text { Personenangaben } \\ \text { B2 } & \text { Gesundheitsfragebogen } \\ \text { B3 } & \text { TAS-20 } \\ \text { B4 } & \text { SCL-K-9 } \\ \text { B5 } & \text { ADS-K } \\ \text { B6 } & \text { STAXI } \\ \text { B7 } & \text { Beschwerdenliste } \\ \text { B8 } & \text { PANAS } \\ \text { B9 } & \text { IES-R } \\ \text { B10 } & \text { STAI-G Form X1 } \\ \text { B11 } & \text { CSS-M } \\ \text { B12 } & \text { Berner Fragebogen } \\ \text { B13 } & \text { MU-BFS } \\ \text { B14 } & \text { PANAS (prä/post) } \\ \text { B15 } & \text { Postexperimenteller Fragebogen } \\ \text { B16 } & \text { Fragebogen Folgeuntersuchung } \\ \text { B17 } & \text { Führungsbenotung }\end{array}$

C Rückmeldung der Teilnehmer 


\section{Anhang A1 - Allgemeine Instruktion-Schreibsitzungen}

Heute und in den nächsten zwei Tagen werden Sie gebeten, 20 Minuten lang über ein bestimmtes Thema zu schreiben. Über das Thema werden Sie etwas später genaueres erfahren.

Bitte nutzen Sie die gesamte Zeit zum Schreiben!!

Falls Ihnen nichts neues mehr einfällt, können Sie wiederholen, was Sei bereits notiert haben.

Machen Sie sich keine Sorgen über Wortwahl, die Rechtschreibung oder Satzbau. Darauf kommt es überhaupt nicht an!!!

Bitte aber sprechen Sie nicht mit anderen Mithäftlingen über das, was Sie innerhalb der Studie tun!

Ihr Schreiben wird vollkommen anonym und vertraulich behandelt werden. Es wird niemand das Geschriebene mit Ihrer Person in Verbindung bringen können. Bitte schreiben Sie Ihren persönlichen Code auf jeden Bogen.

Es kommt manchmal vor, dass Menschen sich nach dem Schreiben etwas aufgewühlt fühlen. Das ist völlig normal. In den meisten Fällen verschwindet das nach spätestens einer Stunde wieder. Wenn Sie dennoch das Bedürfnis haben sollten, über Ihre Gefühle zu sprechen, dann nehmen Sie bitte mit mir (Frau Reinhold-Hurley) oder Frau Schulz auf.

Bitte füllen Sie diesen Fragbogen aus, danach besprechen wir das Schreibthema.

Haben Sie noch Fragen? 


\section{Anhang A2- Einverständniserklärung}

Hiermit erkläre ich mich einverstanden, dass

meine schriftlichen Ausarbeitungen und die von mir ausgefüllten Fragebögen im Rahmen dieses Projektes für wissenschaftliche Zwecke verwendet werden dürfen.

2. gegebenenfalls Einblick in die mich betreffenden Akten der Strafanstalt im Rahmen dieser Studie genommen werden darf.

I ch bin ausreichend darüber informiert worden, dass

1. sich die Doktorandin verpflichtet, alle datenschutzrechtlichen Bestimmungen einzuhalten. Sie ist schweigeverpflichtet und gibt somit keine personenbezogenen Daten an Dritte weiter.

2. eine Auswertung und Veröffentlichung jeglicher Daten nur in anonymisierter Form erfolgt, so dass kein Rückschluss auf die Person möglich ist.

3. Angaben zur Person werden getrennt von Codenummer und den erhobenen Daten und den geschriebenen Essays aufbewahrt.

4. meine Teilnahme freiwillig ist und mir durch die Entscheidung für oder gegen die Teilnahme keine rechtlichen Vor- oder Nachteile entste hen ich jederzeit abbrechen und meine Schreibstücke und Fragebögen zurückfordern kann.

5. ich die Möglichkeit habe, in einem persönlichen Gespräch über mögliche Belastungen durch das Schreiben zu sprechen.

6. es besonders wichtig ist, an der gesamten Studie teilzunehmen und damit auch an der späteren Nachuntersuchung. Außerdem ist es wichtig, nicht mit anderen Mithäftlingen über meine persönlichen Erfahrungen im Rahmen dieses Projektes zu sprechen.

7. ich eine Aufwandsvergütung in Höhe von $10 €$ nach Abschluss der gesamten Untersuchung erhalte.

Ort, Datum Unterschrift 


\section{Anhang A3- Schreibinstruktionen (Gruppe:BE)}

\section{Schreibtag}

Ich bitte Sie, heute und in den nächsten zwei Tagen über ein persönliches Erlebnis zu schreiben, das in Ihrem Leben bisher am belastendsten oder schlimmsten für Sie war.

Sie sollten dabei nicht über Erlebnisse schreiben, die im Zusammenhang mit Ihren Straftaten stehen.

Beim Schreiben sollen Sie dazu Ihre tiefsten Gefühle, Empfindungen und Gedanken erkunden und ausdrücken.

\section{Schreibtag}

Nachdem Sie gestern über ein sehr belastendes Erlebnis geschrieben haben, bitte ich Sie heute fortzufahren. Dabei sollen Sie insbesondere über die Zeit danach und die Folgen schreiben.

Beim Schreiben sollen Sie dazu Ihre tiefsten Gefühle, Empfindungen und Gedanken erkunden und ausdrücken.

\section{Schreibtag}

Heute in der letzten Schreibsitzung möchte ich Sie bitten, sich noch einmal das belastende Erlebnis vor Augen zu führen.

Versuchen Sie heute beim Schreiben das Erlebte abschließend zu bewerten. Wie sehen Sie das Erlebte aus heutiger Sicht?

Dabei sollen Sie Ihre tiefsten Gefühle, Empfindungen und Gedanken erkunden und ausdrücken.

Alles, was Sie schreiben wird streng vertraulich behandelt!

Der Text, den Sie schreiben kann nicht mit Ihnen als Person in Verbindung gebracht werden. 


\section{Anhang A4- Schreibinstruktion (Gruppe: TA)}

\section{Schreibtag}

Ich bitte Sie, heute und in den nächsten zwei Tagen über Situationen zu schreiben, die mit Straftaten, die Sie begangen haben in Zusammenhang stehen.

Die Straftat(-en), über die Sie schreiben, muss nicht notwendigerweise eine sein, für die Sie bereits belangt worden sind.

Beim Schreiben sollen Sie dazu Ihre tiefsten Gefühle, Empfindungen und Gedanken erkunden und ausdrücken.

\section{Schreibtag}

Nachdem Sie gestern über Situationen, die in Zusammenhang mit Ihrer Straftat stehen geschrieben haben, bitte ich Sie heute fortzufahren. Dabei sollen Sie insbesondere über die Zeit danach und die Folgen schreiben.

Beim Schreiben sollen Sie dazu Ihre tiefsten Gefühle, Empfindungen und Gedanken erkunden und ausdrücken.

\section{Schreibtag}

Heute in der letzten Schreibsitzung möchte ich Sie bitten, sich noch einmal Ihre Straftat(-en) vor Augen zu führen.

Versuchen Sie heute beim Schreiben das Erlebte abschließend zu bewerten.

Dabei sollen Sie Ihre tiefsten Gefühle, Empfindungen und Gedanken erkunden und ausdrücken.

Alles, was Sie schreiben wird streng vertraulich behandelt!

Der Text, den Sie schreiben kann nicht mit Ihnen als Person in Verbindung gebracht werden. 


\section{Anhang A5- Schreibinstruktion (Gruppe KG)}

1. Schreibtag

Bitte schreiben Sie heute und in den nächsten zwei Tagen, wie Sie Ihren Zeit verbringen.

Beim heutigen Schreiben sollen Sie beschreiben, was Sie gestern vom Aufstehen bis zum Zubettgehen getan haben.

Bitte seien Sie so objektiv wie möglich und schreiben Sie nicht über Gefühle oder Ihre Meinung, sondern bleiben Sie so sachlich wie möglich. Sie können so detailliert wie möglich schreiben.

\section{Schreibtag}

Nachdem Sie gestern über den Tagesablauf vom Vortag geschrieben haben, bitte ich Sie heute darum, zu beschreiben, was Sie am heutigen Tag seit dem Aufstehen bis jetzt gemacht haben.

Beim Schreiben sollen Sie erneut so objektiv wie möglich beschreiben, was Sie bisher gemacht haben.

\section{Schreibtag}

Heute in der letzten Schreibsitzung möchte ich Sie bitten, so genau wie möglich zu beschreiben, was Sie morgen machen werden.

Beim Schreiben sollen Sie wieder so objektiv wie möglich sein. Bitte schreiben Sie nicht über Gefühle oder Ihre Meinung, sondern bleiben Sie so sachlich wie möglich. Sie können so detailliert wie möglich schreiben.

Alles, was Sie schreiben wird streng vertraulich behandelt!

Der Text, den Sie schreiben kann nicht mit Ihnen als Person in Verbindung gebracht werden. 


\section{Anhang B1- Personenangaben}

1. Wann sind Sie geboren?

2. Wo wurden Sie geboren? Stadt

$\mathrm{BL}$

Ggf. Staatsangehörigkeit, wenn nicht deutsch

3. Wie ist Ihr Familienstand?

ledig $\square$ verheiratet $\square$ feste Partnerschaft $\square$ geschieden $\square$ anderes

\section{Leben Ihre leiblichen Eltern noch?}

$\square \quad$ beide leben noch

$\square \quad$ Mutter verstorben,

Vater verstorben, Jahr Jahr

$\square \quad$ Mutter ist unbekannt $\square$ Vater ist unbekannt

\section{Wo sind Sie hauptsächlich aufgewachsen?}
mit beiden Eltern
Mutter
Vater

$\square$ bei Mutter und Lebenspartner $\square$ bei Vater und Lebenspartnerln

$\square$ bei Verwandten

$\square$ bei Pflege- oder Adoptiveltern

im Heim $\square$ sonstiges

\section{Haben Sie schon einmal im Heim gelebt?}

nein

$\square$ ja

Zeitraum

\section{Was ist Ihr höchster Schulabschluß?}

$\square$ noch keinen $\square$ Sonderschulabschluß $\square$ Hauptschulabschluß

$\square$ Mittlere Reife $\square$ erweiterter Realschulabschluß $\square$ (Fach-)Abitur

\section{Haben Sie bereits eine Berufsausbilung abgeschlossen?}
$\square \quad$ nein, noch nie eine Berufsausbildung begonnen
betriebliche Lehre $\square$ begonnen, aber nicht abgeschlossen
$\square$ abgeschlossen
beruflich-schulische Ausbildung $\square$ begonnen, aber nicht abgeschlossen
Fach-/ Hochschule $\square$ abgeschlossen
$\square$ begonnen, aber nicht abgeschlossen
$\square$ abgeschlossen 


\section{Anhang B1}

9. Sind Sie hier in der JVA in einer Ausbildungs- bzw. Weiterbildungsmaßnahme?

$\square \quad$ nein

$\square$ ja, (genaue Bezeichnung)

10. Haben Sie irgendwann einmal oder nehmen Sie derzeit an einer therapeutischen Maßnahme oder einem Training (z.B. Anti- Aggressionstraining) in der JVA teil?
nein, noch nie
$\square$ ja, derzeit

$\square$ ja, früher (Zeitpunkt, Dauer) (Maßnahme)

\section{Gesundheit und Wohlbefinden}

11. Nehmen Sie regelmäßig Medikamente? Wenn ja, welche?

nein

$\square$ ja,

12. Leiden Sie unter chronischen Krankheiten? Wenn ja, unter welchen?

$\square$ nein $\quad \square$ ja

13. Waren Sie schon einmal in psychotherapeutischer oder psychiatrischer Behandlung?

nein $\square$ ja, in psychother. Behandlung , zuletzt

$\square$ ja, in psychiatr. Behandlung, zuletzt

wenn ja, was war der Anlass?

14. Wurden Sie schon einmal wegen irgendwelcher MedikamentenAlkohol- oder Drogenprobleme behandelt?

$\square$ nein $\square$ ja, einmal $\square$ ja, mehrmals Wann zuletzt? Maßnahme 


\section{Anhang B1}

\section{Angaben zu Inhaftierung und Tat}

15. Gab es in den letzten drei Monaten irgendwelche besonderen Ereignisse oder Veränderungen in Ihrem Leben ? (z.B. Trennung von Partnerin, Umzug, Heirat, Tod eines Verwandten)

Wenn ja, welche waren das?

nein $\square$ ja,

16. Wie war Ihre Stimmung im vergangenen Monat ?

$\square$ sehr gut $\square$ gut $\square$ mittelmäßig $\square$ schlecht $\square$ sehr schlecht

17. Führen Sie regelmäßig ein persönliches Tagebuch ?
ja
$\square$ nein

18. Seit wann sind Sie in dieser Anstalt?

Seit (genaues Datum)

Wann haben Sie Ihre Strafhaft angetreten?

$\mathrm{Am}$ (genaues Datum)

Akteneintrag:

19. Aufgrund welcher Straftaten sind Sie verurteilt worden? (Delikte)

Akteneintrag: 


\section{Anhang B1}

20. Zu welcher Strafe sind Sie verurteilt worden?

$\mathrm{Zu}$

Akteneintrag:

21. Waren Sie zuvor in Untersuchungshaft?

$\square$ nein $\square$ ja, von bis

Offizieller Grund

22. Existieren noch ausstehende Verfahren?

$\square$ nein $\square$ ja, (kurze Beschreibung)

23. Wie belastend ist es für Sie inhaftiert zu sein?

gar nicht

etwas

ziemlich

sehr

24. Haben Sie die Straftat(-en), wegen der Sie verurteilt wurden, überwiegend alleine begangen oder gab es noch andere Beteiligte?
alleine
$\square \quad$ einer von mehreren

25. (t0) Welche Gründe waren Ihrer Meinung nach Auslöser für das Begehen der Straftat?

26. (t0) Wer oder was war Ihrer Meinung nach Schuld an dem Ganzen?

27. Haben Sie zum Tatzeitpunkt unter Alkohol- bzw. Drogeneinfluss gestanden?

$\square$ nein $\quad \square$ ja , unter

$\square$ keine Angabe 


\section{Anhang B1}

28. Hatten Sie früher schon einmal eine Bewährungsstrafe?

$\square$ nein $\square$ ja

29. Waren Sie schon einmal in Jugendarrest?

$\square$ nein $\square$ ja

30. Sind Sie in früheren Verfahren schon mal zu Sanktionen (z.B.

Arbeitsauflagen, Geldbußen, Betreuungsmaßnahmen)

verpflichtet worden?

nein $\square$ ja

31. Wie alt waren Sie als Sie zum ersten Mal straffällig geworden sind? (Alter) (Angabe, ob dafür belangt oder nicht)

\section{Freizeit/Soziale Einbindung}

32. Was machen Sie gern in Ihrer Freizeit?

33. Mit wem verbringen Sie überwiegend Ihre Freizeit?
allein
mit Freunden
mit Partnerln
mit Familie
sonstiges

34. Waren Sie vor Ihrer Inhaftierung Mitglied eines festen Freundeskreises?

$\square$ ja $\square$ nein

35. Besteht zu diesen Freunden/Bekannten noch Kontakt?
ja
nein
teilweise

36. Hatten Sie vor Ihrer Inhaftierung eine feste Freundin?

ja $\square$ nein 


\section{Anhang B1}

37. Falls ja, dauert die Beziehung noch an?

$\square$ ja $\square$ nein

38. Mit wem aus Ihrem Familien- und Freundeskreis haben Sie heute noch Kontakt?

$\square$ Mutter $\quad \square$ Vater $\quad \square$ Geschwister $\quad \square$ Freundin $\square$ Freunden
$\square$ Bekannte $\quad \square$ andere




\section{Anhang B2- Gesundheitsfragebogen}

Bitte beantworten Sie folgenden Fragen bezüglich Ihrer Gesundheit.

1. An wie vielen Tagen waren Sie in den letzten acht Wochen krank? An Tagen

Was hatten Sie?

2. Wie oft waren Sie in den letzten acht Wochen beim Arzt? Arzt Zahnarzt

3. Was war der Anlass?

4. An wie vielen Tagen waren Ihre Alltagsaktivitäten in den letzten acht Wochen durch Krankheit eingeschränkt?

5. Haben Sie in den letzten acht Wochen Alkohol getrunken?

$\square$ nein

$\square \mathrm{ja}$, mal

6. Haben Sie irgendwelche Drogen genommen?

nein

$\square \mathrm{ja}$, mal

6a. Was haben Sie genommen?

(Substanzen) 


\section{Anhang B3 - TAS-20}

Bitte geben sie an, wie sehr jeder der folgenden Feststellungen zustimmen oder nicht, indem Sie ein Kreuz im entsprechenden Kästchen machen. Bitte beantworten Sie jede Frage und geben Sie bitte nur eine Antwort pro Feststellung.

\begin{tabular}{|c|c|c|c|c|c|}
\hline & $\begin{array}{l}\text { stimme } \\
\text { über- } \\
\text { haupt } \\
\text { nicht zu }\end{array}$ & $\begin{array}{l}\text { stimme } \\
\text { teilwei- } \\
\text { se zu }\end{array}$ & $\begin{array}{l}\text { bin } \\
\text { untent- } \\
\text { schieden }\end{array}$ & $\begin{array}{l}\text { stimme } \\
\text { überwei- } \\
\text { gend zu }\end{array}$ & $\begin{array}{l}\text { stimme } \\
\text { völlig } \\
\text { zu }\end{array}$ \\
\hline $\begin{array}{l}\text { 1. Mir ist oft unklar, welche Gefühle } \\
\text { ich gerade habe. }\end{array}$ & & & & & \\
\hline $\begin{array}{l}\text { 2. Es fällt mir schwer, die richtigen } \\
\text { Worte für meine Gefühle zu finden. }\end{array}$ & & & & & \\
\hline $\begin{array}{l}\text { 3. Ich habe körperliche Empfindun- } \\
\text { gen, die sogar Ärzte nicht richtig } \\
\text { verstehen. }\end{array}$ & & & & & \\
\hline $\begin{array}{l}\text { 4. Es fällt mir leicht, meine Gefühle } \\
\text { zu beschreiben. }\end{array}$ & & & & & \\
\hline $\begin{array}{l}\text { 5. Ich ziehe es vor, Probleme zu } \\
\text { analysieren als sie lediglich zu be- } \\
\text { schreiben. }\end{array}$ & & & & & \\
\hline $\begin{array}{l}\text { 6. Wenn ich aufgeregt bin, weiß ich } \\
\text { oft nicht, ob ich traurig, ängstlich } \\
\text { oder wütend bin. }\end{array}$ & & & & & \\
\hline $\begin{array}{l}\text { 7. Ich bin oft über Empfindungen in } \\
\text { meinem Körper verwirtt. }\end{array}$ & & & & & \\
\hline $\begin{array}{l}\text { 8. Ich lasse die Dinger lieber ein- } \\
\text { fach geschehen und versuche nicht } \\
\text { herauszufinden, warum sie gerade } \\
\text { so passiert sind. }\end{array}$ & & & & & \\
\hline $\begin{array}{l}\text { 9. Einige meiner Gefühle kann ich } \\
\text { gar nicht richtig benennen. }\end{array}$ & & & & & \\
\hline $\begin{array}{l}\text { 10. In engem Kontakt mit seinen } \\
\text { Gefühlen zu sein finde ich sehr } \\
\text { wichtig. }\end{array}$ & & & & & \\
\hline $\begin{array}{l}\text { 11. Ich finde es schwierig zu be- } \\
\text { schreiben, was ich für andere Men- } \\
\text { schen empfinde. }\end{array}$ & & & & & \\
\hline $\begin{array}{l}\text { 12. Andere fordern mich auf, meine } \\
\text { Gefühle mehr zu beschreiben. }\end{array}$ & & & & & \\
\hline $\begin{array}{l}\text { 13. Ich weiß nicht, was in mir vor- } \\
\text { geht. }\end{array}$ & & & & & \\
\hline $\begin{array}{l}\text { 14. Ich weiß oft nicht, warum ich } \\
\text { wütend bin. }\end{array}$ & & & & & \\
\hline
\end{tabular}




\begin{tabular}{|l|l|l|l|l|l|}
\hline & $\begin{array}{c}\text { stimme } \\
\text { über- } \\
\text { haupt } \\
\text { nicht zu }\end{array}$ & $\begin{array}{c}\text { stimme } \\
\text { teilwei- } \\
\text { se zu }\end{array}$ & $\begin{array}{c}\text { bin } \\
\text { untent- } \\
\text { schieden }\end{array}$ & $\begin{array}{c}\text { stimme } \\
\text { überwei- } \\
\text { gend zu }\end{array}$ & $\begin{array}{c}\text { stimme } \\
\text { völlig } \\
\text { zu }\end{array}$ \\
\hline $\begin{array}{l}\text { 15. Ich unterhalte mich mit anderen } \\
\text { nicht so gern über ihre Gefühle, } \\
\text { sondern lieber über ihre täglichen } \\
\text { Aktivitäten. }\end{array}$ & & & & \\
\hline $\begin{array}{l}\text { 16. Ich sehe mir lieber „leichte" Un- } \\
\text { terhaltungssendungen als psycho- } \\
\text { logische Dramen an. }\end{array}$ & & & & \\
\hline $\begin{array}{l}\text { 17. Es fällt mir schwer meine in- } \\
\text { nersten Gefühle mitzuteilen, selbst } \\
\text { engen Freunden gegenüber. }\end{array}$ & & & & \\
\hline $\begin{array}{l}\text { 18. Ich kann mich jemandem sogar } \\
\text { in Augenblicken des Schweigens } \\
\text { sehr nah fühlen. }\end{array}$ & & & & & \\
\hline $\begin{array}{l}\text { 19. Ich finde es wichtig auf meine } \\
\text { Gefühle zu achtn, wenn ich persön- } \\
\text { liche Probleme lösen muß. }\end{array}$ & & & & & \\
\hline $\begin{array}{l}\text { 20. Durch die Suche nach verbor- } \\
\text { genen Bedeutungen nimmt man } \\
\text { sich das Vergnügen an Filmen oder } \\
\text { Theaterstücken. }\end{array}$ & & & & & \\
\hline
\end{tabular}




\section{Anhang B4 - SCL-K-9}

Sie finden hier eine Liste von Problemen und Beschwerden, die man manchmal hat. Bitte lesen Sie jede Frage sorgfältig durch und entscheiden Sie, wie sehr Sie in den letzten sieben Tagen durch diese Beschwerden gestört oder bedrängt worden sind.

\begin{tabular}{|c|c|c|c|c|c|}
\hline $\begin{array}{c}\text { Wie seht litten Sie in den letzten sie- } \\
\text { ben Tagen unter....? }\end{array}$ & $\begin{array}{c}\text { über- } \\
\text { haupt } \\
\text { nicht }\end{array}$ & $\begin{array}{c}\text { ein } \\
\text { wenig }\end{array}$ & $\begin{array}{c}\text { ziem- } \\
\text { lich }\end{array}$ & stark & $\begin{array}{l}\text { sehr } \\
\text { stark }\end{array}$ \\
\hline $\begin{array}{l}\text { 1. Gefühlsausbrüchen, gegenüber de- } \\
\text { nen Sie machtlos waren }\end{array}$ & 0 & 1 & 2 & 3 & 4 \\
\hline $\begin{array}{l}\text { 2. dem Gefühl, dass es Ihnen schwer- } \\
\text { fällt, etwas anzufangen }\end{array}$ & 0 & 1 & 2 & 3 & 4 \\
\hline $\begin{array}{l}\text { 3. dem Gefühl, sich zu viele Sorgen } \\
\text { machen zu müssen }\end{array}$ & 0 & 1 & 2 & 3 & 4 \\
\hline 4. Verletzlichkeit in Gefühlsdingen & 0 & 1 & 2 & 3 & 4 \\
\hline $\begin{array}{l}\text { 5.dem Gefühl, dass andere Sie beo- } \\
\text { bachten oder über Sie reden }\end{array}$ & 0 & 1 & 2 & 3 & 4 \\
\hline $\begin{array}{l}\text { 6. dem Gefühl, gespannt oder aufgeregt } \\
\text { zus ein }\end{array}$ & 0 & 1 & 2 & 3 & 4 \\
\hline $\begin{array}{l}\text { 7. Schweregefühl in den Armen oder } \\
\text { den Beinen }\end{array}$ & 0 & 1 & 2 & 3 & 4 \\
\hline $\begin{array}{l}\text { 8. Nervosität, wenn Sie alleine gelassen } \\
\text { werden }\end{array}$ & 0 & 1 & 2 & 3 & 4 \\
\hline $\begin{array}{l}\text { 9. Einsamkeitsgefühlen, selbst wenn } \\
\text { Sie in Gesellschaft sind }\end{array}$ & 0 & 1 & 2 & 3 & 4 \\
\hline
\end{tabular}




\section{Anhang B5 - ADS-K}

Bitte kreuzen Sie bei den folgenden Aussagen die Antwort an, die Ihrem Befinden während der letzten Woche am besten entspricht/ ent-sprochen hat.

\begin{tabular}{|l|l|l|l|l|}
\hline Während der letzten Woche ... & selten & manchmal & öfters & meistens \\
\hline $\begin{array}{l}\text { haben mich Dinge beunruhigt, die mir } \\
\text { sonst nichts ausmachen }\end{array}$ & & & & \\
\hline $\begin{array}{l}\text { konnte ich meine trübsinnige Laune } \\
\text { nicht loswerden }\end{array}$ & & & & \\
\hline hatte ich Mühe, mich zu konzentrieren & & & & \\
\hline war ich deprimiert/niedergeschlagen & & & & \\
\hline war alles anstrengend für mich & & & & \\
\hline $\begin{array}{l}\text { dachte ich, mein Leben sei ein einzi- } \\
\text { ger Fehlschlag }\end{array}$ & & & & \\
\hline hatte ich Angst & & & & \\
\hline habe ich schlecht geschlafen & & & & \\
\hline war ich fröhlich gestimmt & & & & \\
\hline habe ich weniger als sonst geredet & & & & \\
\hline fühlte ich mich einsam & & & & \\
\hline habe ich das Leben genossen & & & & \\
\hline kour ich traurig & & & & \\
\hline
\end{tabular}




\section{Anhang B6 - STAXI}

Im fogenden finden Sie eine Reihe von Feststellungen, mit denen man sich selbst beschreiben kann. Bitte lesen Sie jede Feststellung durhc und wählen Sie aus den vier Antworten diejenige aus, die angibt, wie Sie sich jetzt, d.h. in diesem Moment, fühlen. Es gibt keine richtigen oder falschen Antworten.

\begin{tabular}{|l|c|c|c|c|}
\hline & $\begin{array}{c}\text { über- } \\
\text { haupt } \\
\text { nicht }\end{array}$ & $\begin{array}{c}\text { ein } \\
\text { wenig }\end{array}$ & ziemlich & sehr \\
\hline 1. Ich bin ungehalten & 1 & 2 & 3 & 4 \\
\hline 2. Ich bin wütend & 1 & 2 & 3 & 4 \\
\hline 3. Ich bins sauer & 1 & 2 & 3 & 4 \\
\hline 4. Ich bin enttäuscht & 1 & 2 & 3 & 4 \\
\hline 5. Ich bin zornig & 1 & 2 & 3 & 4 \\
\hline 6. Ich bin aufgebracht & 1 & 2 & 3 & 4 \\
\hline 7. Ich bin schlecht gelaunt & 1 & 2 & 3 & 4 \\
\hline $\begin{array}{l}\text { 8. Ich könnte vor Wut in die Luft ge- } \\
\text { hen }\end{array}$ & 1 & 2 & 3 & 4 \\
\hline 9. Ich bin ärgerlich & 1 & 2 & 3 & 4 \\
\hline 10. Ich könnte laut schimpfen & 1 & 2 & 3 & 4 \\
\hline
\end{tabular}




\section{Anhang B7 - Beschwerdenliste}

Bitte fülle Sie diese Liste sorgfältig aus. Machen Sie ein Kreuz in einer der vier Spalten entsprechend Ihrer Zustimmung bzw. Ablehnung.

Beziehen Sie sich dabei auf die letzten vier Wochen.

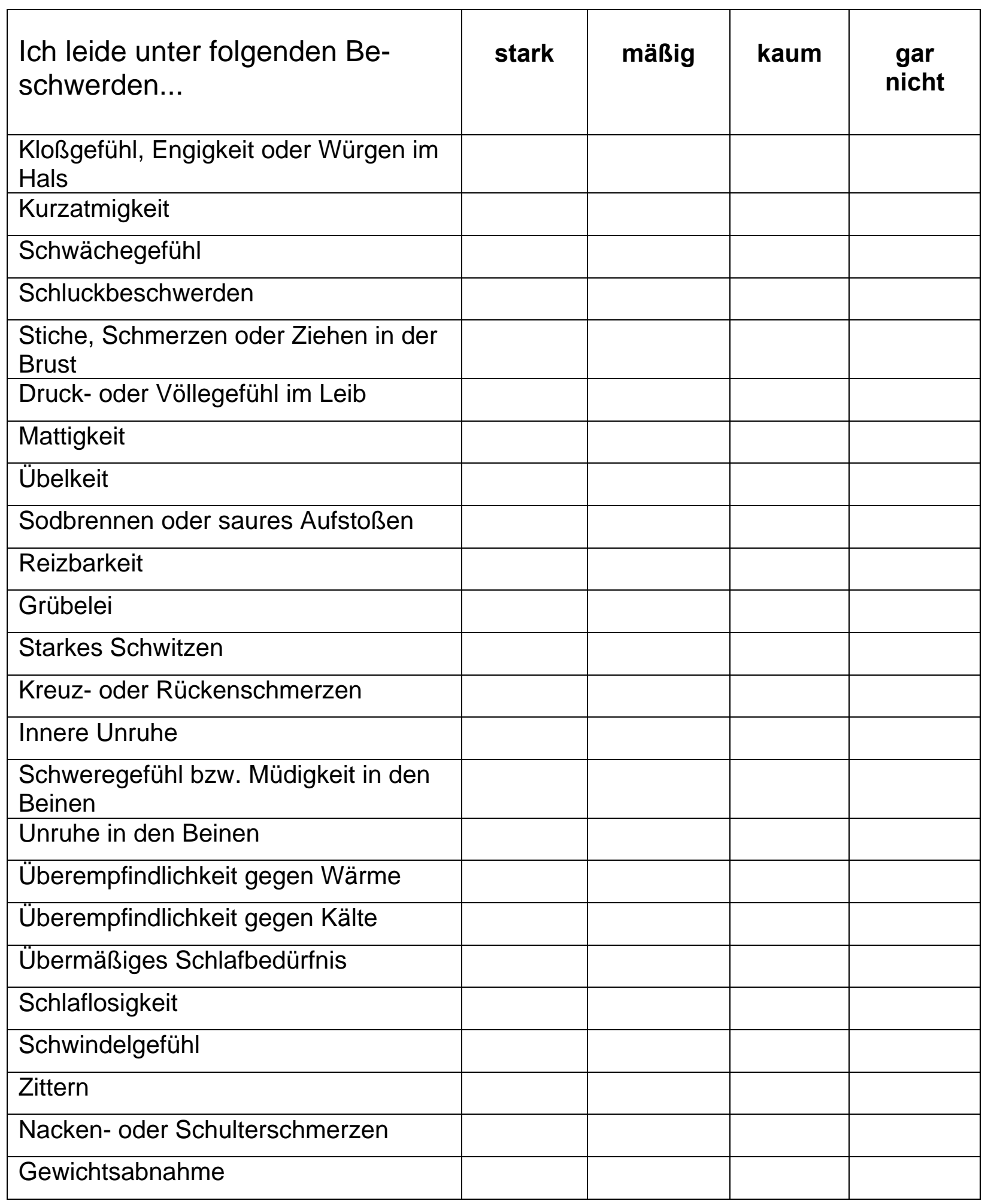




\section{Anhang B8 - PANAS}

Bitte geben Sie an, wie Sie sich im allgemeinen fühlen.

$\begin{array}{cccc}\text { wenig } & \text { ein } & \text { mittel- } & \text { ziemlich } \\ \text { oder } & \text { bißchen } & \text { mäßig } & \\ \text { gar nicht } & & & \end{array}$

\begin{tabular}{|c|c|c|c|c|c|}
\hline aktiv & $\square$ & $\square$ & $\square$ & $\square$ & \\
\hline traurig & $\square$ & $\square$ & $\square$ & $\square$ & \\
\hline interessiert & $\square$ & $\square$ & $\square$ & $\square$ & \\
\hline freudig erregt & $\square$ & $\square$ & & & $\square$ \\
\hline verärgert & $\square$ & $\square$ & $\square$ & $\square$ & \\
\hline stark & $\square$ & $\square$ & $\square$ & $\square$ & \\
\hline schuldig & $\square$ & $\square$ & $\square$ & $\square$ & \\
\hline erschrocken & $\square$ & $\square$ & $\square$ & $\square$ & \\
\hline feindselig & $\square$ & $\square$ & $\square$ & $\square$ & \\
\hline angeregt & $\square$ & $\square$ & $\square$ & $\square$ & \\
\hline stolz & $\square$ & $\square$ & $\square$ & $\square$ & \\
\hline gereizt & $\square$ & $\square$ & $\square$ & $\square$ & \\
\hline begeistert & $\square$ & $\square$ & $\square$ & $\square$ & \\
\hline beschämt & $\square$ & $\square$ & $\square$ & $\square$ & \\
\hline wach & $\square$ & $\square$ & $\square$ & $\square$ & \\
\hline nervös & $\square$ & $\square$ & $\square$ & $\square$ & \\
\hline entschlossen & $\square$ & $\square$ & $\square$ & $\square$ & \\
\hline aufmerksam & $\square$ & $\square$ & $\square$ & $\square$ & \\
\hline durcheinander & $\square$ & $\square$ & $\square$ & $\square$ & \\
\hline ängstlich & $\square$ & $\square$ & $\square$ & $\square$ & \\
\hline
\end{tabular}




\section{Anhang B9 - IES-R}

Denken Sie bitte an das Ereignis, dass Sie beschrieben haben.

Geben Sie im Folgenden an, wie Sie in der letzten Woche zu diesem Ereignis gestanden haben, indem Sie für jede der folgenden Reaktionen ankreuzen, wie häufig diese bei Ihnen aufgetreten ist.

\begin{tabular}{|c|c|c|c|c|}
\hline & $\begin{array}{c}\text { überhaupt } \\
\text { nicht }\end{array}$ & selten & manchmal & oft \\
\hline $\begin{array}{l}\text { Immer, wenn ich an das Ereignis erinnert } \\
\text { wurde, kehrten die Gefühle wieder. }\end{array}$ & & & & \\
\hline $\begin{array}{l}\text { Ich hatte Schwierigkeiten, nachts durchzu- } \\
\text { schlafen. }\end{array}$ & & & & \\
\hline $\begin{array}{l}\text { Andere Dinge erinnerten mich immer wieder } \\
\text { daran. }\end{array}$ & & & & \\
\hline Ich fühlte mich reizbar und ärgerlich. & & & & \\
\hline $\begin{array}{l}\text { Ich versuchte, mich nicht aufzuregen, wenn } \\
\text { ich daran dachte oder daran erinnert wurde. }\end{array}$ & & & & \\
\hline $\begin{array}{l}\text { Auch ohne es zu beabsichtigen, musste ich } \\
\text { daran denken. }\end{array}$ & & & & \\
\hline $\begin{array}{l}\text { Es kam mir so vor, als ob es gar nicht ge- } \\
\text { schehen wäre oder irgendwie unwirklich war. }\end{array}$ & & & & \\
\hline $\begin{array}{l}\text { Ich versuchte, Erinnerungen daran aus dem } \\
\text { Weg zu gehen. }\end{array}$ & & & & \\
\hline $\begin{array}{l}\text { Bilder, die mit dem Ereignis zu tun hatten, } \\
\text { kamen mir plötzlich in den Sinn. }\end{array}$ & & & & \\
\hline Ich war leicht reizbar und schreckhaft. & & & & \\
\hline Ich versuchte, nicht daran zu denken. & & & & \\
\hline $\begin{array}{l}\text { Ich merkte zwar, dass meine Gefühle durch } \\
\text { das Ereignis noch sehr aufgewühlt waren, } \\
\text { aber ich beschäftigte mich nicht mit ihnen. }\end{array}$ & & & & \\
\hline $\begin{array}{l}\text { Die Gefühle, die das Ereignis in mir auslöste, } \\
\text { waren ein bisschen wie abgestumpft. }\end{array}$ & & & & \\
\hline $\begin{array}{l}\text { Ich stellte fest, dass ich handelte oder fühlte, } \\
\text { als ob ich in die Zeit (des Ereignisses) zu- } \\
\text { rückversetzt sei. }\end{array}$ & & & & \\
\hline Ich konnte nicht einschlafen. & & & & \\
\hline
\end{tabular}




\begin{tabular}{|l|l|l|l|l|}
\hline & $\begin{array}{c}\text { überhaupt } \\
\text { nicht }\end{array}$ & selten & manchmal & oft \\
\hline $\begin{array}{l}\text { Es kam mir so vor, dass die Gefühle, die mit } \\
\text { dem Ereignis zusammenhingen, plötzlich für } \\
\text { kurze Zeit viel heftiger wurden. }\end{array}$ & & & & \\
\hline $\begin{array}{l}\text { Ich versuchte, das Ereignis aus meiner Erin- } \\
\text { nerung zu streichen. }\end{array}$ & & & & \\
\hline Es fiel mir schwer, mich zu konzentrieren. & & & \\
\hline $\begin{array}{l}\text { Die Erinnerung daran lösten bei mir körperli- } \\
\text { che Reaktionen aus, wie Schwitzen, Atem- } \\
\text { not, Schwindel oder Herzklopfen. }\end{array}$ & & & & \\
\hline $\begin{array}{l}\text { Ich träumte davon. } \\
\text { aufmerksam und hellhörig. }\end{array}$ & & & & \\
\hline Ich empfand mich sersuchte, nicht darüber zu sprechen. & & & & \\
\hline
\end{tabular}




\section{Anhang B10 - STAI-G Form X1}

Im folgenden Fragebogen finden Sie eine Reihe von Feststellungen, mit denen man sich selbst beschreiben kann. Bitte lesen Sie jede Feststellung durch und wählen Sie aus den vier Antworten diejenige aus, die angibt, wie Sie sich jetzt, d.h. in diesem Moment fühlen.

\begin{tabular}{|l|l|l|l|l|}
\hline & $\begin{array}{c}\text { über- } \\
\text { haupt } \\
\text { nicht }\end{array}$ & $\begin{array}{c}\text { ein } \\
\text { wenig }\end{array}$ & ziemlich & sehr \\
\hline 1. Ich bin ruhig. & & & & \\
\hline 2. ich fühle mich geborgen. & & & & \\
\hline 3. Ich fühle mich angespannt. & & & & \\
\hline 4. Ich bin bekümmert. & & & & \\
\hline 5. Ich bin gelöst. & & & & \\
\hline 6. Ich bin aufgeregt. & & & & \\
\hline 7. Ich bin besorgt, dass etwas schief \\
gehen könnte.
\end{tabular}




\section{Anhang B11 - CSS-M (TLV u. ICO)}

Bitte lesen Sie jede der folgenden Aussagen sorgfältig durch und geben Sie an, wie Sie zu diesen Aussagen stehen.

Bitte denken Sie daran: Es gibt keine richtigen oder falschen Antworten!

Stimmt $\underset{\text { entschlossen }}{\text { un- }} \begin{array}{r}\text { stimmt } \\ \text { nicht }\end{array}$

1. Jemand wie ich muss manchmal das Gesetz

$\mathrm{O}$

$\mathrm{O}$

$\mathrm{O}$ brechen, um im Leben vorwärts zu kommen.

$\mathrm{O}$

$\mathrm{O}$

Die meisten erfolgreichen Leute haben das Gesetz gebrochen, um im Leben vorwärts zu kommen.

3. Man sollte immer den Gesetzen folgen,

$\mathrm{O}$

O

O auch wenn es einen daran hindert, im Leben vorwärts zu kommen.

4. Es ist in Ordnung das Gesetz zu brechen, solange man nicht erwischt wird.

5. Die meisten Menschen würden Straftaten begehen, wenn sie nicht erwischt würden.

$\mathrm{O}$

O

$\mathrm{O}$

6. Es gibt nie einen guten Grund, das Gesetz

O

O

$\mathrm{O}$

zu brechen.

7. Ein hungriger Mensch hat das Recht zu stehlen.

O

$\mathrm{O}$

$\mathrm{O}$

8. Es ist in Ordnung Gesetze zu umgehen, solange man sie nicht wirklich bricht.

Bitte umblättern つ 


\section{Stimmt un- stimmt entschlossen nicht}

9. Man sollte nur solche Gesetze achten, die vernünftig sind.

10. Man ist verrückt, für seinen Lebensunterhalt

$\mathrm{O}$

O

$\mathrm{O}$ zu arbeiten, wenn es auch einen leichteren Weg gibt - selbst wenn das bedeutet, die Gesetze zu brechen.

11. Leute, die das Gesetz gebrochen haben, haben

O $\quad 0$

O die gleichen Einstellungen zum Leben wie ich.

12. Ich bin lieber mit Leuten zusammen, die das Gesetz achten als mit Leuten, die das Gesetz brechen.

13. Ich bin einem professionellen Kriminellen ähnlicher $\mathrm{O}$

$\mathrm{O}$

$\mathrm{O}$ als Leuten, die ab und zu das Gesetz brechen.

14. Leute, die mit dem Gesetz in Konflikt geraten sind, sind mir ähnlicher als Leute, die keine Schwierigkeiten mit dem Gesetz haben.

15. Ich habe sehr wenig gemeinsam mit Leuten, die nie das Gesetz brechen.

16. Jemand, der das Gesetz bricht, kann nicht mein Freund sein.

Vielen Dank! 


\section{Anhang B12 - Berner Fragebogen}

Wie schätzen Sie im Nachhinein Ihre Straftat ein?

Bitte beurteilen Sie die folgenden Aussagen und kreuzen das jeweilige Kästchen an.

\begin{tabular}{|c|c|c|c|c|}
\hline & $\begin{array}{c}\begin{array}{c}\text { Stimmt } \\
\text { nicht }\end{array} \\
1\end{array}$ & $\begin{array}{c}\text { Stimmt } \\
\text { eher } \\
\text { nicht } \\
2\end{array}$ & $\begin{array}{c}\begin{array}{c}\text { Stimmt } \\
\text { eher }\end{array} \\
3\end{array}$ & $\begin{array}{c}\text { Stimmt } \\
4\end{array}$ \\
\hline $\begin{array}{l}\text { Meinem Freund/meinen Freunden die Treue zu halten } \\
\text { ist mir wichtiger, als nach irgendwelchen Gesetzen zu } \\
\text { handeln. }\end{array}$ & 1 & 2 & 3 & 4 \\
\hline $\begin{array}{l}\text { Ich hatte wirklich keine Ahnung, was ich durch meine } \\
\text { Tat bei anderen angerichtet habe. }\end{array}$ & 1 & 2 & 3 & 4 \\
\hline $\begin{array}{l}\text { Ich kann gar nicht verstehen, warum ich alleine schuldig } \\
\text { sein soll, denn andere haben sehr viel dazu beigetra- } \\
\text { gen, dass es soweit gekommen ist. }\end{array}$ & 1 & 2 & 3 & 4 \\
\hline $\begin{array}{l}\text { Wenn jemand durch mich Schaden erlitten hat, so ge- } \\
\text { schah dies ohne Absicht. }\end{array}$ & 1 & 2 & 3 & 4 \\
\hline $\begin{array}{l}\text { Diejenigen, die mich hierher gebracht haben, sind auch } \\
\text { nicht besser als ich: Aber auf mir hacken die alle rum. }\end{array}$ & 1 & 2 & 3 & 4 \\
\hline $\begin{array}{l}\text { Mir wurde sehr übel mitgespielt und deshalb hatte ich } \\
\text { auch das Recht „zurückzuschlagen“. }\end{array}$ & 1 & 2 & 3 & 4 \\
\hline $\begin{array}{l}\text { Die Gesellschaft trägt zum grossen Teil Mitschuld dar- } \\
\text { an, wenn Menschen wie ich auf die schiefe Bahn gera- } \\
\text { ten. }\end{array}$ & 1 & 2 & 3 & 4 \\
\hline $\begin{array}{l}\text { Die ganze Situation war so schwierig und kompliziert, } \\
\text { dass ich die Folgen meines Handelns nicht übersehen } \\
\text { konnte. }\end{array}$ & 1 & 2 & 3 & 4 \\
\hline $\begin{array}{l}\text { Ich habe nach dem Prinzip „Im Leben gilt das Recht } \\
\text { des Stärkeren“ gehandelt. Wenn man nicht zuerst } \\
\text { schlägt, so wird man von den anderen geschlagen. }\end{array}$ & 1 & 2 & 3 & 4 \\
\hline $\begin{array}{l}\text { Bei meiner Straftat habe ich wie unter Zwang gehan- } \\
\text { delt, ich konnte einfach nicht anders. }\end{array}$ & 1 & 2 & 3 & 4 \\
\hline $\begin{array}{l}\text { Was ich getan habe, tun heutzutage so viele, dass es } \\
\text { auf einen mehr oder weniger gar nicht mehr ankommt. }\end{array}$ & 1 & 2 & 3 & 4 \\
\hline $\begin{array}{l}\text { Ich wusste sehr genau, was ich tat und hätte mich je- } \\
\text { derzeit umbesinnen können. }\end{array}$ & 1 & 2 & 3 & 4 \\
\hline $\begin{array}{l}\text { Ich kann mir meine Tat nur so erklären, dass mich der } \\
\text { Teufel oder ein böser Geist geritten hat. }\end{array}$ & 1 & 2 & 3 & 4 \\
\hline Bitte umblättern! & & & & \\
\hline
\end{tabular}




\begin{tabular}{|c|c|c|c|c|}
\hline & $\begin{array}{c}\text { Stimmt } \\
\text { nicht } \\
1\end{array}$ & $\begin{array}{l}\text { Stimmt } \\
\text { eher } \\
\text { nicht } \\
2\end{array}$ & $\begin{array}{l}\text { Stimmt } \\
\text { eher } \\
3\end{array}$ & $\begin{array}{c}\text { Stimmt } \\
4\end{array}$ \\
\hline $\begin{array}{l}\text { Ich bin nicht verantwortlich für das, was ich getan habe: } \\
\text { Die äusseren Umstände (Arbeitslosigkeit, Familienver- } \\
\text { hältnisse etc.) waren schuld daran. }\end{array}$ & 1 & 2 & 3 & 4 \\
\hline $\begin{array}{l}\text { Ich habe es eigentlich gut gemeint, aber dann ist alles } \\
\text { schiefgegangen. }\end{array}$ & 1 & 2 & 3 & 4 \\
\hline $\begin{array}{l}\text { Wenn ich ein ganz bestimmtes Ziel verfolge, dann pas- } \\
\text { siert es manchmal, dass ich die Konsequenzen meines } \\
\text { Tuns aus den Augen verliere. }\end{array}$ & 1 & 2 & 3 & 4 \\
\hline $\begin{array}{l}\text { Der von mir angerichtete Schaden wurde von anderen } \\
\text { masslos übertrieben. }\end{array}$ & 1 & 2 & 3 & 4 \\
\hline $\begin{array}{l}\text { Wenn ich nicht erwischt worden wäre, dann hätte ich } \\
\text { mir auch keine weiteren Gedanken über meine Tat ma- } \\
\text { chen müssen. }\end{array}$ & 1 & 2 & 3 & 4 \\
\hline $\begin{array}{l}\text { Nach meiner Tat konnte ich mir selbst nicht mehr erklä- } \\
\text { ren, wie ich das habe machen können. }\end{array}$ & 1 & 2 & 3 & 4 \\
\hline $\begin{array}{l}\text { Das Urteil war gerecht, die Strafe entspricht der Schwe- } \\
\text { re meiner Schuld. }\end{array}$ & 1 & 2 & 3 & 4 \\
\hline $\begin{array}{l}\text { Da die „Grossen“ ungestraft gegen Gesetze verstossen } \\
\text { können, ist nicht einzusehen, warum sich die „Kleinen“ } \\
\text { an das Gesetz halten sollen. }\end{array}$ & 1 & 2 & 3 & 4 \\
\hline $\begin{array}{l}\text { Ich hatte Pech mit dem Richter: Andere werden für die } \\
\text { gleiche Tat weniger oder gar nicht bestraft. }\end{array}$ & 1 & 2 & 3 & 4 \\
\hline $\begin{array}{l}\text { Irgendwie hat sich bei der Straftat mein Handeln ver- } \\
\text { selbständigt, ich habe mich einfach nicht mehr verstan- } \\
\text { den. }\end{array}$ & 1 & 2 & 3 & 4 \\
\hline $\begin{array}{l}\text { Bei einer besseren Verteidigung oder Fürsprache hätte } \\
\text { ich deutlich weniger Strafe bekommen, wäre vielleicht } \\
\text { sogar freigesprochen worden. }\end{array}$ & 1 & 2 & 3 & 4 \\
\hline $\begin{array}{l}\text { Ich habe niemandem Unrecht getan oder irgendeinen } \\
\text { Schaden zugefügt, auch wenn ich vielleicht gegen ein } \\
\text { Gesetz verstossen habe. }\end{array}$ & 1 & 2 & 3 & 4 \\
\hline $\begin{array}{l}\text { Wenn man es genau betrachtet, dann ist meine Straftat } \\
\text { durch eine Verkettung unglücklicher Umstände ent- } \\
\text { standen. }\end{array}$ & 1 & 2 & 3 & 4 \\
\hline $\begin{array}{l}\text { Es könnte gut sein, dass meine Tat in naher Zukunft } \\
\text { oder in einem anderen Land schon gar nicht mehr be- } \\
\text { straft wird. }\end{array}$ & 1 & 2 & 3 & 4 \\
\hline $\begin{array}{l}\text { Meine Gefängnisstrafe ist zu hoch: Sie steht in keinem } \\
\text { Verhältnis zur Tat. }\end{array}$ & 1 & 2 & 3 & 4 \\
\hline $\begin{array}{l}\text { Ob ich schuldig bin oder nicht, ist mir egal: } \\
\text { Wichtig ist nur, wie es für mich weitergeht. }\end{array}$ & 1 & 2 & 3 & 4 \\
\hline $\begin{array}{l}\text { Wenn ich bereits als Kind und Jugendlicher günstigere } \\
\text { Bedingungen gehabt hätte, so wäre ich sicher nicht } \\
\text { straffällig geworden. }\end{array}$ & 1 & 2 & 3 & 4 \\
\hline
\end{tabular}

Vielen Dank! ; 


\section{Anhang B13 - MU-BFS}

Inwieweit stimmen Sie persönlich den folgenden Aussagen zu?

\begin{tabular}{|c|c|c|c|c|}
\hline 1. Straftaten zu begehen lohnt sich oft, weil .... & $\begin{array}{c}\text { trifft } \\
\text { nicht zu }\end{array}$ & $\begin{array}{c}\text { trifft } \\
\text { kaum } \\
\text { zu }\end{array}$ & $\begin{array}{c}\text { trifft } \\
\text { eher zu }\end{array}$ & $\begin{array}{c}\text { trifft } \\
\text { völlig } \\
\text { zu }\end{array}$ \\
\hline \multicolumn{5}{|l|}{$\begin{array}{l}\text {...die anderen sich ja auch bereichern, } \\
\text { wo sie können }\end{array}$} \\
\hline \multicolumn{5}{|l|}{$\begin{array}{l}\text {... das Risiko erwischt zu werden, } \\
\text { ziemlich klein ist }\end{array}$} \\
\hline \multicolumn{5}{|l|}{... es Spaß macht } \\
\hline \multicolumn{5}{|l|}{... der Gewinn höher ist als die Strafe } \\
\hline \multicolumn{5}{|l|}{$\begin{array}{l}\text {... man ja doch keine andere Chance hat, etwas zu bekom } \\
\text { men }\end{array}$} \\
\hline \multicolumn{5}{|l|}{$\begin{array}{l}\text {.. man manchmal die eigenen Interessen mit Gewalt durch- } \\
\text { setzen muss }\end{array}$} \\
\hline \multicolumn{5}{|l|}{$\begin{array}{l}\text {... man denen oben zeigen kann, dass man cleverer ist als } \\
\text { die }\end{array}$} \\
\hline $\begin{array}{l}\text {... man den Freunden beweisen kann, dass man mutig und } \\
\text { cool ist }\end{array}$ & & & & \\
\hline
\end{tabular}

weiter auf der nächsten Seite! 


\section{Anhang B13}

\begin{tabular}{|c|c|c|c|c|}
\hline $\begin{array}{l}\text { 2. Straftaten sollte man besser nicht begehen, } \\
\text { weil... }\end{array}$ & $\begin{array}{c}\text { trifft } \\
\text { nicht zu }\end{array}$ & $\begin{array}{c}\text { trifft } \\
\text { kaum } \\
\text { zu }\end{array}$ & $\begin{array}{c}\text { trifft } \\
\text { eher zu }\end{array}$ & $\begin{array}{l}\text { trifft } \\
\text { völlig } \\
\text { zu }\end{array}$ \\
\hline ... man ja doch meist erwischt wird & & & & \\
\hline ... der Gewinn die Strafe niemals ausgleicht & & & & \\
\hline ... man das eben einfach nicht tun soll & & & & \\
\hline ... es wichtig ist, die Gesetze zu beachten & & & & \\
\hline $\begin{array}{l}\text {... es wichtig ist, die Regeln zu beachten, die auch andere } \\
\text { beachten sollen }\end{array}$ & & & & \\
\hline ... es wertvoll ist, ein gutes Gewissen zu haben & & & & \\
\hline ... man anderen schadet, die nichts dafür können & & & & \\
\hline $\begin{array}{l}\text {... es wichtig ist, anderen (z.B. Kindern) mit gutem Beispiel } \\
\text { voranzugehen }\end{array}$ & & & & \\
\hline ... ich mir selbst dabei schade & & & & \\
\hline
\end{tabular}

Vielen Dank! 


\section{Anhang B14- PANAS-prä/post}

Bitte geben Sie an, wie Sie sich jetzt in diesem Moment fühlen.

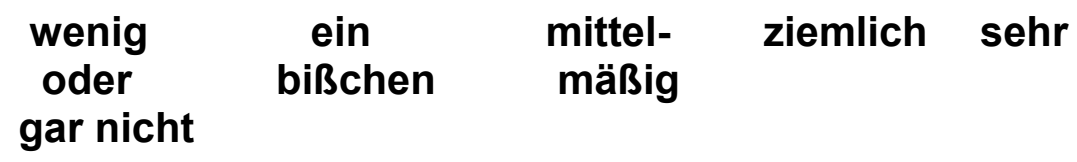

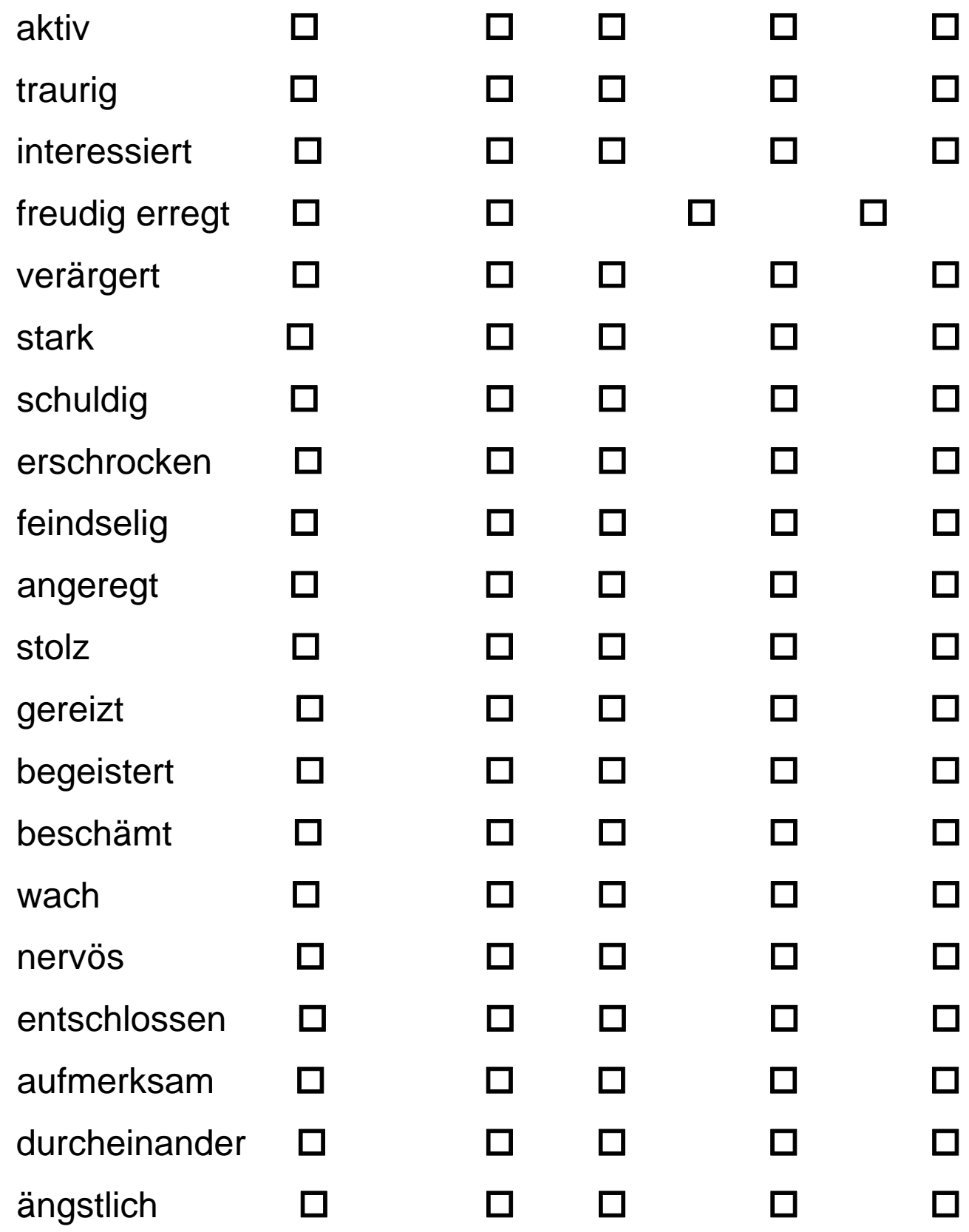




\section{Anhang B15 - Post-FB}

Bitte beantworten Sie folgende Fragen...

- Wie persönlich ist das, was Sie heute geschrieben haben?

$\begin{array}{ccccccc}1 & 2 & 3 & 4 & 5 & 6 & \begin{array}{c}7 \\ \text { sehr persönlich }\end{array} \\ \text { nicht persönlich } & & & & & \end{array}$

- Wie stark haben Sie Ihre Gefühle ausgedrückt?

$\begin{array}{ccccccc}1 & 2 & 3 & 4 & 5 & 6 & \begin{array}{c}7 \\ \text { sehr stark }\end{array} \\ \text { gar nicht } & & & & & & \end{array}$

- Hat Sie das Schreiben heute belastet?

$\begin{array}{ccccccc}1 & 2 & 3 & 4 & 5 & 6 & \begin{array}{c}7 \\ \text { sehr stark }\end{array}\end{array}$

- Hat Sie das Schreiben heute erleichtert?

$\begin{array}{ccccccc}1 & 2 & 3 & 4 & 5 & 6 & \begin{array}{c}7 \\ \text { sehr stark }\end{array}\end{array}$

- Ist es Ihnen schwer gefallen, beim Schreiben Ihre Gefühle auszudrücken?

$\begin{array}{ccccccc}1 & 2 & 3 & 4 & 5 & 6 & \begin{array}{c}7 \\ \text { nicht schwer }\end{array}\end{array}$

- Wie sehr hat Sie das Beschriebene damals belastet ?

$\begin{array}{ccccccc}1 & 2 & 3 & 4 & 5 & 6 & \begin{array}{c}7 \\ \text { sehr stark }\end{array}\end{array}$

Wie stark fühlen Sie sich heute noch dadurch belastet?

$\begin{array}{ccccccc}1 & 2 & 3 & 4 & 5 & 6 & \begin{array}{c}7 \\ \text { sehr stark }\end{array}\end{array}$




\section{Anhang B15}

- Wie oft haben Sie mit anderen bisher über das Thema, über das Sie heute geschrieben haben, gesprochen?

$\begin{array}{ccccccc}1 & 2 & 3 & 4 & 5 & 6 & \begin{array}{c}7 \\ \text { sehr oft }\end{array}\end{array}$

- Haben sie es bisher bewusst vermieden, über das Thema zu sprechen?
12
3
4
5
$6 \quad 7$
nicht vermieden
sehr vermieden

- Haben Sie bereits im Rahmen einer therapeutischen Maßnahme über das beschriebene Thema gesprochen?
Nein
$\mathrm{Ja}$
wenn ja, wann war
das?

- Wie lange liegt das, über das Sie geschrieben haben zurück ?

Ca. Jahre/ bzw. Monate 


\section{Anhang B16 - FB-Folgeuntersuchung}

Bitte beantworten Sie folgende Fragen bezogen auf die letzten 2 Monate, also den Zeitraum nach dem Schreib-Projekt!

- Wie oft haben Sie an das gedacht, worüber Sie geschrieben haben?

$\begin{array}{ccccccc}1 & 2 & 3 & 4 & 5 & 6 & \begin{array}{c}7 \\ \text { sehr oft }\end{array} \\ \text { gar nicht } & & & & & & \end{array}$

- Wie oft haben Sie seitdem mit anderen über das Thema gesprochen?

$\begin{array}{ccccccc}1 & 2 & 3 & 4 & 5 & 6 & \begin{array}{c}7 \\ \text { sehr oft }\end{array} \\ \text { gar nicht } & & & & & & \end{array}$

- Wie sehr hat das Schreiben Sie positiv beeinflusst?

$\begin{array}{ccccccc}1 & 2 & 3 & 4 & 5 & 6 & \begin{array}{c}7 \\ \text { sehr stark }\end{array} \\ \text { gar nicht } & & & & & & \end{array}$

Bitte beschreiben Sie kurz, was sich ggf. verändert hat

- Wie sehr hat das Schreiben Sie negativ beeinflusst?

$\begin{array}{ccccccc}1 & 2 & 3 & 4 & 5 & 6 & \begin{array}{c}7 \\ \text { sehr stark }\end{array} \\ \text { gar nicht } & & & & & & \end{array}$

Bitte beschreiben Sie kurz, was sich ggf. verändert hat:

- Wie bewerten Sie das Projekt im nachhinein?

$\begin{array}{ccccccc}1 & 2 & 3 & 4 & 5 & 6 & \begin{array}{c}7 \\ \text { sehr gut }\end{array} \\ \text { sehr schlecht } & & & & & \end{array}$

- Führen Sie seit der Teilnahme an dem Projekt ein persönliches Tagebuch? $\mathrm{Ja}$ Nein

- Haben Sie noch Anmerkungen zum Projekt? Was hat Ihnen besonders gefallen oder auch nicht gefallen? 


\section{Anhang B17- Führungsbenotung}

DATUM: $\quad$ CODE: $\quad$ Nr.: $\quad$ GRUPPE:

Erstuntersuchung: Schreibsitzungen:

Folgeuntersuchung:

Inhaftierung: $\quad$ Entlassung nach 2/3:

- Führungsbenotung (Stufenüberprüfungsverfahren) seit Inhaftierung:

1. Note (gesamt): Datum:

Schule/Arbeit: Erziehungsgruppe: Sport/Freizeit: Stufe:

2. Note (gesamt): Datum:

Schule/Arbeit: Erziehungsgruppe: Sport/Freizeit:

Höherstufung:

3. Note (gesamt): Datum:

Schule/Arbeit: Erziehungsgruppe: Sport/Freizeit:

Höherstufung:

4. Note (gesamt): Datum:

Schule/Arbeit: Erziehungsgruppe: Sport/Freizeit:

Höherstufung:

5. Note (gesamt): Datum:

Schule/Arbeit: Erziehungsgruppe: Sport/Freizeit:

Höherstufung: 


\section{Anhang B17}

- Anhörungsvermerke und Verstöße laut Akte zum Zeitpunkt t1:

1. am:

2. am:

3. am:

4. am:

- Sonstiges: 


\section{Anhang C - Rückmeldung der Teilnehmer \\ Rückmeldung/Anmerkungen der Teilnehmer am Schreibprojekt \\ (Gruppenzugehörigkeit in Klammern)}

„Die Gesellschaft war nett.“ (TA)

„War sehr gut.“ (KG)

„Dass man Sachen los werden konnte, wodrüber man sonst nicht reden kann.“ (KG)

„Sehr nett.“ (TA)

„Das ich über Sachen geschrieben habe mit dem ich sonst mit keinem drüber rede.“ (BE)

„War zur falschen Zeit bei mir wegen Sterbefall.“ (BE)

„War alles ok." (KG)

„Ich weiß ehrlich gesagt nicht, was es bringt oder nicht bringt.“ (KG)

„Alles sehr gut.“ (TA)

„Sachen, über die man sich sonst keine Gedanken macht.“ (TA)

„Das man ohne Folgen über sachen schreiben kann für die man sonst bestraft werden könn-

te." (TA)

„Das freundliche auftreten der Mitarbeiterin.“ (BE)

„Die Unterhaltung hat mir gefallen.“ (KG)

„Positives miteinander“ (BE)

„Mir gefallen nicht diese Fragebogen aber war schon witzig mit der Lady.“ (TA).

„Mann musste zuviel ankreuzen.“ (KG)

„Mir hat nicht gefallen das ich über mein Opa geredet habe oder geschrieben habe.“ (BE)

„Das Projekt war zu kurz." (TA)

„Is mal was anderes." (KG)

„Mir hat gefallen mal darüber zu reden.“(BE)

„Das man nicht von Beamten usw. beobachtet wird und man seine Ruhe hat beim Nachdenken."(KG)

„Wahr ganz in ordnung. (BE)

„Man war abgelenkt.“(BE)

„Das Schreiben hat mir gefallen.“(KG)

„Es war so weit alles o.k. nichts negatives."(TA)

„War eigentlich ganz normal.“(KG)

„Mir hat es Spaß gemacht, und mir hat das Schreibprojekt positiv geholfen! Danke.“(BE) 